\title{
RESERVATÓRIOS METÁLICOS ELEVADOS PARA APLICAÇÃO NA ÁREA DE PROCESSOS DA INDÚSTRIA SUCRO-ALCOOLEIRA
}

\author{
RENATO CELINI BADIALE
}

Dissertação apresentada à Escola de Engenharia de São Carlos, da Universidade de São Paulo, como parte dos requisitos para obtenção do Título de Mestre em Engenharia de Estruturas.

ORIENTADOR : Prof. Dr. José Jairo de Sáles

São Carlos 
Ficha catalográfica preparada pela Seção de Tratamento da Inform ação do Serviço de Biblioteca - EESC-USP

Badiale, Renato Celini

Reservatórios metálicos elevados para aplicação

na área de processos da indústria sucro-alcooleira

/ Renato Celini Badiale. -- São Carlos, 1997.

Dissertação (Mestrado). -- Escola de Engenharia

de São Carlos-Universidade de São Paulo, 1997.

Área: Engenharia de Estruturas

Orientador: Prof. Dr. José Jairo de Sáles

1. Reservatórios metálicos. 2. Cálculo

estrutural. 3. Cascas de revolução. I. Título. 
Aos meus pais e irmã

À Célia, esposa e amiga

Aos meus filhos Ligia,

Lucas e Rafael 


\section{AGRADECIMENTOS}

À Deus, pela oportunidade e pelas pessoas que colocou em minha caminhada.

Aos colegas do curso de pós-graduação pela acolhida e pelo convívio agradável.

Ao pessoal da secretaria, biblioteca e demais funcionários do Departamento de Estruturas pela colaboração.

Aos funcionários do LaMEM pela amizade fraterna.

À todos os professores do curso de pós-graduação pela paciência.

Aos professores Maximiliano Malite, Carlito Calil Jr. pelo apoio e incentivo.

Ao meu orientador professor José Jairo de Sáles pela experiência transmitida, por compreender minhas falhas e limitações, e principalmente, pela franqueza e amizade.

$\grave{A}$ todos os meus familiares, minha eterna gratidão por estarem sempre ao meu lado 


\section{SUMÁRIO}

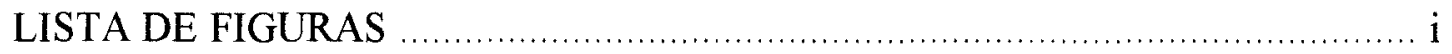

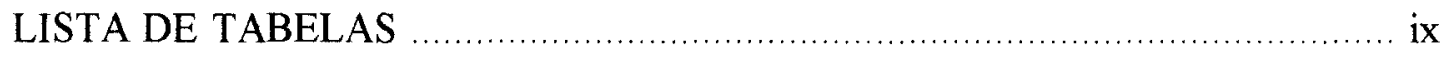

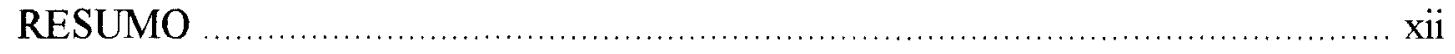

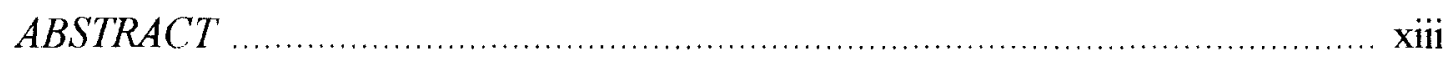

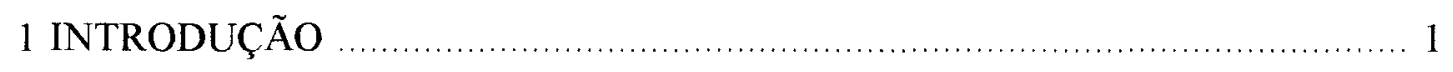

2 EQUACIONAMENTO DAS EXPRESSÕES TEÓRICAS _........................ 11

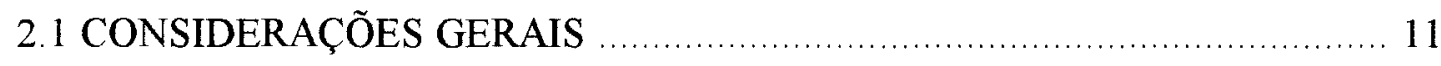

2.2 CASCA CILÍNDRICA _................................................................ 14

2.2.1 CASCA CILÍNDRICA COM BORDA INFERIOR ENGASTADA ............ 19

2.2.2 CASCA CILÍNDRICA COM BORDA INFERIOR APOIADA FIXA ........ 22

2.2.3 TENSÕES TEÓRICAS DE MEMBRANA …...................................... 24

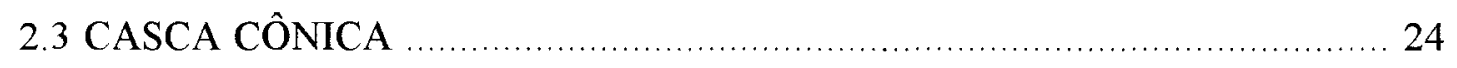

2.3.1 CASCA CÔNICA COM BORDA SUPERIOR ENGASTADA .................. 31

2.3.2 CASCA CÔNICA COM BORDA SUPERIOR APOIADA FIXA …........... 35

2.3.3 TENSÕES TEÓRICAS DE MEMBRANA ....................................... 37

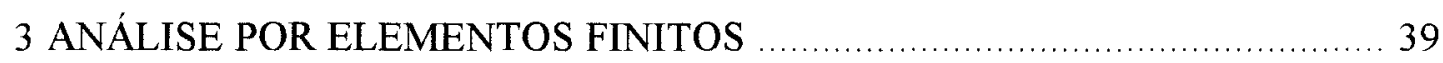

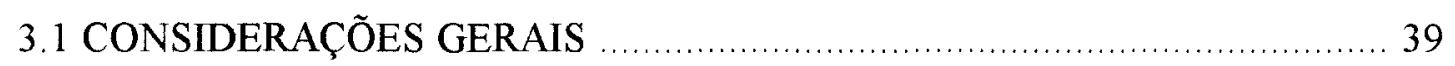

3.2 CODIFICAÇÃO PARA CASCA CILÍNDRICA …................................. 41

3.2.1 ARQUIVO PARA ENTRADA DE DADOS .................................... 43

3.3 CODIFICAÇÃO PARA CASCA CÔNICA ............................................ 49

3.3.1 ARQUIVO PARA ENTRADA DE DADOS ......................................... 50

4 ESTRUTURA "TÍPICA" ANALISADA - COMENTÁRIOS GERAIS ............. 55

5 CALCULANDO A ESTRUTURA "TÍPICA" ......................................... 60 
5.1 CONSIDERAÇÕES GERAIS

5.2 CALCULANDO E OBTENDO RESULTADOS PARA A CASCA CILÍNDRICA 63

5.2.1 CASCA CILÍNDRICA COM BORDA INFERIOR ENGASTADA ..............64

5.2.2 CASCA CILÍNDRICA COM BORDA INFERIOR APOIADA FIXA …...... 74

5.3 CALCULANDO E OBTENDO RESULTADOS PARA A CASCA

CÔNICA INCLINAÇÃO $\theta r=30^{\circ} \mathrm{COM}$ A HORIZONTAL 83

5.3.1 CASCA CÔNICA COM BORDA SUPERIOR ENGASTADA 84

5.3.2 CASCA CÔNICA COM BORDA SUPERIOR APOIADA FIXA 96

5.4 CALCULANDO E OBTENDO RESULTADOS PARA A CASCA

CÔNICA INCLINAÇÃO $\theta \mathrm{r}=45^{\circ} \mathrm{COM}$ A HORIZONTAL 106

5.4. I CASCA CÔNICA COM BORDA SUPERIOR ENGASTADA 107

5.4.2 CASCA CÔNICA COM BORDA SUPERIOR APOIADA FIXA 118

5.5 CALCULANDO E OBTENDO RESULTADOS PARA A CASCA

CÔNICA INCLINAÇÃO $\theta \mathrm{r}=60^{\circ} \mathrm{COM}$ A HORIZONTAL 128

5.5.1 CASCA CÔNICA COM BORDA SUPERIOR ENGASTADA 129

5.5.2 CASCA CÔNICA COM BORDA SUPERIOR APOIADA FIXA 140

6 CONCLUSÕES 150

7 BIBLIOGRAFIA 154 


\section{LISTA DE FIGURAS}

FIGURA 1 - Equipamentos da alimentação de cana ........................................ 2

FIGURA 2 - Equipamento da extração de caldo : "terno"de moenda .................... 2

FIGURA 3 - Transportador metálico distribuidor de bagaço : seção transversal e vista lateral ................................................... 3

FIGURA 4 - Equipamentos da fabricação de açúcar e alcool .............................. 3

FIGURA 5 - Cascas cilíndrica e cônica ….................................................. 4

FIGURA 6 - Foto de dorna de fermentação de usina de açúcar na região de Catanduva - S.P. ....................................................... 5

FIGURA 7 - Foto de decantador com fundo sustentado por casca cônica de concreto ............................................................. 7

FIGURA 8 - Seção transversal da "viga anel" .............................................. 7

FIGURA 9 - Fundo cônico com reforço tipo "grelha circular/radial metálica" ......................................................................... 8

FIGURA 10 - Foto onde se pode visualisar imperfeição inicial do corpo cilíndrico .......................................................... 9

FIGURA 11 - Casca cilíndrica de revolução sob ação de pressão hidrostática

FIGURA 12 - Esquema para as forças e momentos distribuídos na borda vinculada

FIGURA 13 - Esquema para deslocamentos radiais e rotações ........................... 20

FIGURA 14 - Esquema para os momentos solicitantes ................................. 21

FIGURA 15 - Esquema para as tensões atuantes ...................................... 22 
FIGURA 16 - Casca cônica de revolução sob ação de pressão

hidrostática 25

FIGURA 17 - Esquema para as forças e momentos distribuídos na borda superior vinculada 30

FIGURA 18 - Esquema para deslocamentos radiais e rotações 32

FIGURA 19 - Esquema para os momentos solicitantes 33

FIGURA 20 - Esquema para as forças solicitantes 34

FIGURA 21 - Esquema para as tensões atuantes 35

FIGURA 22 - Esquema geral para modelo da casca cilíndrica 42

FIGURA 23 - Esquema da geração dos nós 44

FIGURA 24 - Esquema dos esforços fornecidos na saída de dados para opção "O=0" ("O" igual a zero) 46

FIGURA 25 - Esquema dos esforços fornecidos na saída de dados para opção " $\mathrm{O}=1$ " para as faces interna (top) e externa (bot) do elemento 47

FIGURA 26 - Esquema da geração dos elementos 48

FIGURA 27 - Esquema da geração de nós 52

FIGURA 28 - Esquema da geração dos elementos 54

FIGURA 29 - Esquema do reservatório metálico elevado analisado com fundo cônica a 30,45 e 60 graus com a horizontal 57

FIGURA 30 - Esquema para compreensão da existência de quatro parâmetros por nó

FIGURA 31 - Gráficos para os valores dos deslocamentos radiais $\mathrm{We}(\mathrm{z})$ ao longo do meridiano da superficie média da casca cilíndrica 65

FIGURA 32 - Gráficos para os valores das rotações $\phi \mathrm{e}(\mathrm{z})$ ao longo do meridiano da superfície média da casca cilíndrica 66

FIGURA 33 - Gráficos para os valores dos momentos ao longo dos paralelos $\mathrm{M} 1 \mathrm{e}(\mathrm{z})$ da superfície média da casca cilíndrica 67

FIGURA 34 - Gráficos para os valores dos momentos ao longo dos meridianos M2e(z) da superficie média da casca cilíndrica 68 
FIGURA 35 - Gráficos para os valores das tensões ao longo dos meridianos da superficie interna $\sigma l \operatorname{ein}(\mathrm{z})$ da casca cilíndrica

FIGURA 36 - Gráficos para os valores das tensões ao longo dos meridianos da superficie externa $\sigma$ leex $(\mathrm{z})$ da casca cilíndrica 70

FIGURA 37 - Gráficos para os valores das tensões ao longo dos paralelos da superficie interna $\sigma 2 \operatorname{ein}(\mathrm{z})$ da casca cilíndrica 71

FIGURA 38 - Gráficos para os valores das tensões ao longo dos paralelos da superficie externa $\sigma 2 \operatorname{eex}(\mathrm{z})$ da casca cilíndrica

FIGURA 39 - Gráfico comparativo para os valores das tensões ao longo dos paralelos da casca cilíndrica : $\sigma 2 \mathrm{~m}(\mathrm{z}), \sigma 2 \mathrm{ein}(\mathrm{z})$ e $\sigma 2 \operatorname{eex}(\mathrm{z})$

FIGURA 40 - Gráficos para os valores dos deslocamentos radiais $\mathrm{Wa}(\mathrm{z})$ ao longo do meridiano da superficie média da casca cilíndrica 75

FIGURA 41 - Gráficos para os valores das rotações $\phi a(z)$ ao longo do meridiano da superficie média da casca cilíndrica

FIGURA 42 - Gráficos para os valores dos momentos ao longo dos paralelos $\mathrm{Mla}(\mathrm{z})$ da superfície média da casca cilíndrica 77

FIGURA 43 - Gráficos para os valores dos momentos ao longo dos meridianos $\mathrm{M} 2 \mathrm{a}(\mathrm{z})$ da superficie média da casca cilíndrica

FIGURA 44 - Gráficos para os valores das tensões ao longo dos meridianos da superfície interna $\sigma \mathrm{lain}(\mathrm{z})$ da casca cilíndrica

FIGURA 45 - Gráficos para os valores das tensões ao longo dos meridianos da superficie externa $\sigma$ laex $(z)$ da casca cilíndrica 79

FIGURA 46 - Gráficos para os valores das tensões ao longo dos paralelos da superficie interna $\sigma 2$ ain $(\mathrm{z})$ da casca cilíndrica 81

FIGURA 47 - Gráficos para os valores das tensões ao longo dos paralelos da superfície externa $\sigma 2 \mathrm{aex}(\mathrm{z})$ da casca cilíndrica

FIGURA 48 - Gráfico comparativo para os valores das tensões ao longo dos paralelos da casca cilíndrica : $\sigma 2 \mathrm{~m}(\mathrm{z}), \sigma 2 \operatorname{ain}(\mathrm{z})$ e $\sigma 2 \mathrm{aex}(\mathrm{z})$ 82 
FIGURA 49 - Gráficos para os valores dos deslocamentos radiais $\xi \mathrm{e}(\mathrm{z})$ ao longo do meridiano da superficie média da casca cônica

FIGURA 50 - Gráficos para os valores das rotações $\phi \mathrm{e}(\mathrm{z})$ ao longo do meridiano da superficie média da casca cônica 86

FIGURA 51 - Gráficos para os valores dos momentos ao longo dos paralelos Mle(z) da superficie média da casca cônica 88

FIGURA 52 - Gráficos para os valores dos momentos ao longo dos meridianos $\mathrm{M} 2 \mathrm{e}(\mathrm{z})$ da superficie média da casca cônica 88

FIGURA 53 - Gráficos para os valores das tensões ao longo dos meridianos da superficie interna $\sigma$ lein $(z)$ da casca cônica

FIGURA 54 - Gráficos para os valores das tensões ao longo dos meridianos da superficie externa $\sigma$ leex $(z)$ da casca cônica 90

FIGURA 55 - Gráficos para os valores das tensões ao longo dos paralelos da superficie interna $\sigma 2 \operatorname{ein}(\mathrm{z})$ da casca cônica 92

FIGURA 56 - Gráficos para os valores das tensões ao longo dos paralelos da superficie externa $\sigma 2 \operatorname{eex}(\mathrm{z})$ da casca cônica 92

FIGURA 57 - Gráfico comparativo para os valores das tensões ao longo dos meridianos da casca cônica : $\sigma \operatorname{lm}(z), \sigma l \operatorname{ein}(z)$ e $\sigma \operatorname{leex}(z)$ 94

FIGURA 58 - Gráfico comparativo para os valores das tensões ao longo dos paralelos da casca cônica : $\sigma 2 \mathrm{~m}(\mathrm{z}), \sigma 2 \operatorname{ein}(\mathrm{z})$ e $\sigma 2 \operatorname{eex}(\mathrm{z})$

FIGURA 59 - Gráficos para os valores dos deslocamentos radiais $\xi a(z)$ ao longo do meridiano da superfície média da casca cônica 97

FIGURA 60 - Gráficos para os valores das rotações $\phi a(z)$ ao longo do meridiano da superficie média da casca cônica 97

FIGURA 61 - Gráficos para os valores dos momentos ao longo dos paralelos Mla(z) da superfície média da casca cônica 99

FIGURA 62 - Gráficos para os valores dos momentos ao longo dos meridianos $\mathrm{M} 2 \mathrm{a}(\mathrm{z})$ da superficie média da casca cônica 99 
FIGURA 63 - Gráficos para os valores das tensões ao longo dos meridianos da superficie interna $\sigma$ lain(z) da casca cônica 101

FIGURA 64 - Gráficos para os valores das tensões ao longo dos meridianos da superficie externa $\sigma$ laex(z) da casca cônica

FIGURA 65 - Gráficos para os valores das tensões ao longo dos paralelos da superfície interna $\sigma 2$ ain(z) da casca cônica 103

FIGURA 66 - Gráficos para os valores das tensões ao longo dos paralelos da superficie externa $\sigma 2 \mathrm{aex}(\mathrm{z})$ da casca cônica

FIGURA 67 - Gráfico comparativo para os valores das tensões ao longo dos meridianos da casca cônica : $\sigma \operatorname{lm}(\mathrm{z}), \sigma \mathrm{lain}(\mathrm{z})$ e $\sigma \mathrm{laex}(\mathrm{z})$ 104

FIGURA 68 - Gráfico comparativo para os valores das tensões ao longo dos paralelos da casca cônica : $\sigma 2 \mathrm{~m}(\mathrm{z}), \sigma 2 \mathrm{ain}(\mathrm{z})$ e $\sigma 2 \mathrm{aex}(\mathrm{z})$

FIGURA 69 - Gráficos para os valores dos deslocamentos radiais $\xi$ e(z) ao longo do meridiano da superfície média da casca cônica

FIGURA 70 - Gráficos para os valores das rotações $\phi \mathrm{e}(\mathrm{z})$ ao longo do meridiano da superfície média da casca cônica 108

FIGURA 71 - Gráficos para os valores dos momentos ao longo dos paralelos M1e(z) da superficie média da casca cônica

FIGURA 72 - Gráficos para os valores dos momentos ao longo dos meridianos $\mathrm{M} 2 \mathrm{e}(\mathrm{z})$ da superficie média da casca cônica

FIGURA 73 - Gráficos para os valores das tensões ao longo dos meridianos da superfície interna $\sigma$ lein(z) da casca cônica

FIGURA 74 - Gráficos para os valores das tensões ao longo dos meridianos da superficie externa $\sigma$ leex(z) da casca cônica

FIGURA 75 - Gráficos para os valores das tensões ao longo dos paralelos da superficie interna $\sigma 2 \operatorname{ein}(z)$ da casca cônica 114

FIGURA 76 - Gráficos para os valores das tensões ao longo dos paralelos da superficie externa $\sigma 2 \operatorname{eex}(z)$ da casca cônica 
FIGURA 77 - Gráfico comparativo para os valores das tensões ao longo dos meridianos da casca cônica : $\sigma \operatorname{lm}(\mathrm{z}), \sigma \operatorname{lein}(\mathrm{z})$ e $\sigma \operatorname{leex}(\mathrm{z})$

FIGURA 78 - Gráfico comparativo para os valores das tensões ao longo dos paralelos da casca cônica : $\sigma 2 \mathrm{~m}(\mathrm{z}), \sigma 2 \operatorname{ein}(\mathrm{z})$ e $\sigma 2 \operatorname{eex}(\mathrm{z})$

FIGURA 79 - Gráficos para os valores dos deslocamentos radiais $\xi \mathrm{a}(\mathrm{z})$ ao longo do meridiano da superfície média da casca cônica

FIGURA 80 - Gráficos para os valores das rotações $\phi a(z)$ ao longo do meridiano da superficie média da casca cônica

FIGURA 81 - Gráficos para os valores dos momentos ao longo dos paralelos Mla(z) da superfície média da casca cônica

FIGURA 82 - Gráficos para os valores dos momentos ao longo dos meridianos $\mathrm{M} 2 \mathrm{a}(\mathrm{z})$ da superficie média da casca cônica

FIGURA 83 - Gráficos para os valores das tensões ao longo dos meridianos da superfície interna $\sigma$ lain(z) da casca cônica

FIGURA 84 - Gráficos para os valores das tensões ao longo dos meridianos da superficie externa $\sigma$ laex(z) da casca cônica

FIGURA 85 - Gráficos para os valores das tensões ao longo dos paralelos da superficie interna $\sigma 2$ ain(z) da casca cônica

FIGURA 86 - Gráficos para os valores das tensões ao longo dos paralelos da superficie externa $\sigma 2 \mathrm{aex}(\mathrm{z})$ da casca cônica

FIGURA 87 - Gráfico comparativo para os valores das tensões ao longo dos meridianos da casca cônica : $\sigma \operatorname{lm}(z), \sigma \operatorname{lin}(z)$ e $\sigma \operatorname{laex}(z)$ 126

FIGURA 88 - Gráfico comparativo para os valores das tensões ao longo dos paralelos da casca cônica : $\sigma 2 \mathrm{~m}(\mathrm{z}), \sigma 2 \mathrm{ain}(\mathrm{z})$ e $\sigma 2 \mathrm{aex}(\mathrm{z})$

FIGURA 89 - Gráficos para os valores dos deslocamentos radiais $\xi \mathrm{e}(\mathrm{z})$ ao longo do meridiano da superfície média da casca cônica 130

FIGURA 90 - Gráficos para os valores das rotações $\phi \mathrm{e}(\mathrm{z})$ ao longo do meridiano da superficie média da casca cônica 130

FIGURA 91 - Gráficos para os valores dos momentos ao longo dos 
paralelos Mle(z) da superficie média da casca cônica

FIGURA 92 - Gráficos para os valores dos momentos ao longo dos meridianos $\mathrm{M} 2 \mathrm{e}(\mathrm{z})$ da superficie média da casca cônica

FIGURA 93 - Gráficos para os valores das tensões ao longo dos meridianos da superfície interna $\sigma$ lein(z) da casca cônica

FIGURA 94 - Gráficos para os valores das tensões ao longo dos meridianos da superficie externa $\sigma$ leex $(\mathrm{z})$ da casca cônica

FIGURA 95 - Gráficos para os valores das tensões ao longo dos paralelos da superfície interna $\sigma 2 \operatorname{ein}(z)$ da casca cônica 136

FIGURA 96 - Gráficos para os valores das tensões ao longo dos paralelos da superficie externa $\sigma 2 \operatorname{eex}(z)$ da casca cônica

FIGURA 97 - Gráfico comparativo para os valores das tensões ao longo dos meridianos da casca cônica : $\sigma \mathrm{lm}(\mathrm{z}), \sigma \operatorname{lein}(\mathrm{z})$ e $\sigma \operatorname{leex}(\mathrm{z})$

FIGURA 98 - Gráfico comparativo para os valores das tensões ao longo dos paralelos da casca cônica : $\sigma 2 \mathrm{~m}(\mathrm{z}), \sigma 2 \operatorname{ein}(\mathrm{z})$ e $\sigma 2 \operatorname{eex}(\mathrm{z})$

FIGURA 99 - Gráficos para os valores dos deslocamentos radiais $\xi \mathrm{a}(\mathrm{z})$ ao longo do meridiano da superfície média da casca cônica

FIGURA 100 - Gráficos para os valores das rotações $\phi a(z)$ ao longo do meridiano da superficie média da casca cônica

FIGURA 101 - Gráficos para os valores dos momentos ao longo dos paralelos $\mathrm{Mla}(\mathrm{z})$ da superficie média da casca cônica

FIGURA 102 - Gráficos para os valores dos momentos ao longo dos meridianos $\mathrm{M} 2 \mathrm{a}(\mathrm{z})$ da superfície média da casca cônica

FIGURA 103 - Gráficos para os valores das tensões ao longo dos meridianos da superfície interna $\sigma$ lain(z) da casca cônica

FIGURA 104 - Gráficos para os valores das tensões ao longo dos meridianos da superficie externa $\sigma$ laex $(z)$ da casca cônica 145

FIGURA 105 - Gráficos para os valores das tensões ao longo dos paralelos 
da superfície interna $\sigma 2$ ain $(z)$ da casca cônica 147

FIGURA 106 - Gráficos para os valores das tensões ao longo dos paralelos da superficie externa $\sigma 2 \mathrm{aex}(\mathrm{z})$ da casca cônica

FIGURA 107 - Gráfico comparativo para os valores das tensões ao longo dos meridianos da casca cônica : $\sigma 1 \mathrm{~m}(\mathrm{z}), \sigma$ lain(z) e $\sigma \operatorname{laex}(\mathrm{z})$ 148

FIGURA 108 - Gráfico comparativo para os valores das tensões ao longo dos paralelos da casca cônica : $\sigma 2 \mathrm{~m}(\mathrm{z}), \sigma 2 \mathrm{ain}(\mathrm{z})$ e $\sigma 2 \mathrm{aex}(\mathrm{z})$ 149 


\section{LISTA DE TABELAS}

TABELA 1 - Valores dos deslocamentos radiais $\mathrm{We}(\mathrm{z})$ e rotações $\phi \mathrm{e}(\mathrm{z})$ 64

TABELA 2 - Valores dos momentos ao longo dos paralelos Mle(z) e dos meridianos M2e(z) 66

TABELA 3 - Valores para as tensões ao longo dos meridianos da superficie interna $\sigma$ lein(z) e externa $\sigma$ leex(z) da casca 68

TABELA 4 - Valores para as tensões ao longo dos paralelos da superfície interna $\sigma 2 \operatorname{ein}(z)$ e externa $\sigma 2 \operatorname{eex}(z)$ da casca 70

TABELA 5 - Valores para as tensões teóricas de membrana ao longo dos meridianos $\sigma \operatorname{lm}(z)$ e paralelos $\sigma 2 \mathrm{~m}(\mathrm{z})$ da superfície média da casca 72

TABELA 6 - Valores dos deslocamentos radiais $\mathrm{Wa}(\mathrm{z})$ e rotações $\phi \mathrm{a}(\mathrm{z})$ 74

TABELA 7 - Valores dos momentos ao longo dos paralelos Mla(z) e dos meridianos $\mathrm{M} 2 \mathrm{a}(\mathrm{z})$ 76

TABELA 8 - Valores para as tensões ao longo dos meridianos da superficie interna $\sigma$ lain(z) e externa $\sigma$ laex(z) da casca 78

TABELA 9 - Valores para as tensões ao longo dos paralelos da superfície interna $\sigma 2 \operatorname{ain}(z)$ e externa $\sigma 2 \mathrm{aex}(\mathrm{z})$ da casca 80

TABELA 10 - Valores dos deslocamentos radiais $\xi \mathrm{e}(\mathrm{z})$ e rotações $\phi \mathrm{e}(\mathrm{z})$ 84

TABELA 11 - Valores dos momentos ao longo dos paralelos M1e(z) e dos meridianos $\mathrm{M} 2 \mathrm{e}(\mathrm{z})$ 86

TABELA 12 - Valores para as tensões ao longo dos meridianos da superficie interna $\sigma$ lein $(z)$ e externa $\sigma l \operatorname{eex}(z)$ da casca 89 
TABELA 13 - Valores para as tensões ao longo dos paralelos da superficie interna $\sigma 2 \operatorname{ein}(z)$ e externa $\sigma 2 \operatorname{eex}(z)$ da casca 91

TABELA 14 - Valores para as tensões teóricas de membrana ao longo dos meridianos $\sigma 1 \mathrm{~m}(\mathrm{z})$ e paralelos $\sigma 2 \mathrm{~m}(\mathrm{z})$ da superficie média da casca 93

TABELA 15 - Valores dos deslocamentos radiais $\xi \mathrm{a}(\mathrm{z})$ e rotações $\phi \mathrm{a}(\mathrm{z})$ 96

TABELA 16 - Valores dos momentos ao longo dos paralelos M1a(z) e dos meridianos $\mathrm{M} 2 \mathrm{a}(\mathrm{z})$ 98

TABELA 17 - Valores para as tensões ao longo dos meridianos da superficie interna $\sigma$ lain(z) e externa $\sigma$ laex(z) da casca 100

TABELA 18 - Valores para as tensões ao longo dos paralelos da superficie interna $\sigma 2$ ain $(z)$ e externa $\sigma 2 \operatorname{aex}(z)$ da casca 102

TABELA 19 - Valores dos deslocamentos radiais $\xi \mathrm{e}(\mathrm{z})$ e rotações $\phi \mathrm{e}(\mathrm{z})$ 107

TABELA 20 - Valores dos momentos ao longo dos paralelos M1 e(z) e dos meridianos M2e(z) 109

TABELA 21 - Valores para as tensões ao longo dos meridianos da superfície interna $\sigma$ lein $(z)$ e externa $\sigma l \operatorname{eex}(z)$ da casca

TABELA 22 - Valores para as tensões ao longo dos paralelos da superfície interna $\sigma 2 \operatorname{ein}(z)$ e externa $\sigma 2 \operatorname{eex}(z)$ da casca

TABELA 23 - Valores para as tensões teóricas de membrana ao longo dos meridianos $\sigma 1 \mathrm{~m}(\mathrm{z})$ e paralelos $\sigma 2 \mathrm{~m}(\mathrm{z})$ da superficie média da casca

TABELA 24 - Valores dos deslocamentos radiais $\xi \mathrm{a}(\mathrm{z})$ e rotações $\phi \mathrm{a}(\mathrm{z})$ 118

TABELA 25 - Valores dos momentos ao longo dos paralelos M1a(z) e dos meridianos $\mathrm{M} 2 \mathrm{a}(\mathrm{z})$

TABELA 26 - Valores para as tensões ao longo dos meridianos da superficie interna $\sigma$ lain(z) e externa $\sigma \mathrm{laex}(\mathrm{z})$ da casca 
TABELA 27 - Valores para as tensões ao longo dos paralelos da superficie interna $\sigma 2 \operatorname{ain}(\mathrm{z})$ e externa $\sigma 2 \mathrm{aex}(\mathrm{z})$ da casca 124

TABELA 28 - Valores dos deslocamentos radiais $\xi \mathrm{e}(\mathrm{z})$ e rotações $\phi \mathrm{e}(\mathrm{z})$ 129

TABELA 29 - Valores dos momentos ao longo dos paralelos M1e(z) e dos meridianos $\mathrm{M} 2 \mathrm{e}(\mathrm{z})$

TABELA 30 - Valores para as tensões ao longo dos meridianos da superficie interna $\sigma$ lein(z) e externa $\sigma$ leex(z) da casca

TABELA 31 - Valores para as tensões ao longo dos paralelos da superficie interna $\sigma 2 \operatorname{ein}(z)$ e externa $\sigma 2 \operatorname{eex}(z)$ da casca

TABELA 32 - Valores para as tensões teóricas de membrana ao longo dos meridianos $\sigma \operatorname{lm}(\mathrm{z})$ e paralelos $\sigma 2 \mathrm{~m}(\mathrm{z})$ da superficie média da casca 137

TABELA 33 - Valores dos deslocamentos radiais $\xi \mathrm{a}(\mathrm{z})$ e rotações $\phi \mathrm{a}(\mathrm{z})$ 140

TABELA 34 - Valores dos momentos ao longo dos paralelos M1a(z) e dos meridianos M2a(z)

TABELA 35 - Valores para as tensões ao longo dos meridianos da superfície interna $\sigma$ lain $(z)$ e externa $\sigma$ laex $(z)$ da casca

TABELA 36 - Valores para as tensões ao longo dos paralelos da superficie interna $\sigma 2 \operatorname{ain}(z)$ e externa $\sigma 2 \mathrm{aex}(\mathrm{z})$ da casca 146

TABELA 37 - Valores das diferenças absolutas máximas para os parâmetros da casca cônica com a borda superior engastada e inclinação $\theta \mathrm{r}=10$ [graus] 152 


\section{RESUMO}

BADIALE, R.C. (1997). Reservatórios metálicos elevados para a aplicação na área de processos da indístria sucro-alcooleira. São Carlos, 1997. 158p. Dissertação (Mestrado) - Escola de Engenharia de São Carlos, Universidade de São Paulo.

Em doze anos de atuação profissional no desenvolvimento de projetos específicos para a indústria sucro-alcooleira, observou-se a pouca informação encontrada para o projeto de equipamentos utilizados neste setor, sendo de grande utilidade aos profissionais que nele atuam, algo introdutório, para auxiliar na análise estrutural de reservatórios metálicos elevados utilizados na fabricação de açúcar e alcool. Objetivou-se, utilizar a teoria de estruturas em casca de revolução e programa "comercial" de análise de estruturas por elementos finitos, como ferramentas, no cálculo estrutural de cascas cilíndrica e cônica que compõem um equipamento com dimensões "típicas" utilizadas na área de processos da indústria sucro-alcooleira. $\mathrm{O}$ estudo de forma comparativa com o método dos elementos finitos permitiu calibrar a solução teórica aproximada desenvolvida. As equações obtidas, para cascas cilíndricas e cônicas com bordas engastadas ou apoiadas fixas sob a ação de pressão hidrostática, podem ser utilizadas para prever o seu comportamento estrutural, desde que observadas hipóteses e recomendações básicas. Mesmo distantes da simulação do comportamento real das cascas, já que em campo, encontram-se inúmeros outros parâmetros não considerados neste trabalho, procurou-se apresentar um melhor esboço do comportamento estrutural das mesmas com equacionamento acessível aos engenheiros não acadêmicos envolvidos com problemas de projeto estrutural.

Palavras-chave : Reservatórios metálicos; cálculo estrutural; cascas de revolução. 


\begin{abstract}
BADIALE, R.C. Elevated steel tanks used in sugar and alcohol industry processes area. São Carlos, 1997. 158p. Dissertação (Mestrado) - Escola de Engenharia de São Carlos, Universidade de São Paulo.

In twelve years of professional performance in the development of specific projects for the sugar and alcohol industry, the little information for equipments used in this sector design was observed, and it would be of great usefulness to the design engineers of this area, something introductory in the structural analysis of elevated steel tanks used in the production of sugar and alcohol. This work had as objective to use the shell of revolution theory and "commercial" programs for finite element analysis of structures, as tools, in the calculation of cylindrical and conical shells that compose a typical "dimensions" equipment used in the sugar industry processes area. The study in a comparative way with the finite elements method allowed to gauge the approximate theoretical solution developed. The equations developed for cylindrical and conical shells with fixed or supported borders under liquid pressure action, can be used to anticipate the structural behavior of the same ones, since fundamental hypotheses and basic recommendations wil have observed. In spite of far from the real behavior simulation of the shells, because of the countless others in field parameters existence and that had not considered in this work, we tried to present a better sketch of the structural behavior of the shells with useful equations for the non academics' engineers involved with structural design problems.
\end{abstract}

Keywords : Stell tanks; structural design; shell of revolution. 


\section{INTRODUÇÃo}

Em doze anos de atuação no desenvolvimento de projetos específicos para a indústria sucro-alcooleira, nem sempre foi possível ter certeza das hipóteses e critérios adotados, tendo muitas vezes que adaptar-se às limitações técnicas, devido a pouca informação encontrada na indústria, para o dimensionamento $\mathrm{e}$ projeto de equipamentos para as aplicações do setor.

A falta de um melhor entrosamento entre indústrias e universidades em nosso país, também foi, e continua sendo, responsável pela dificuldade de acesso do engenheiro não acadêmico aos novos estudos e pesquisas desenvolvidas sobre assuntos que é chamado a resolver no seu dia-a-dia.

Algumas vezes também deparou-se com problemas devido ao caráter ainda "coronelista" de certas usinas, que ainda não poderiam ser chamadas de "indústrias", o que levava a algumas surpresas, vendo equipamentos sendo utilizados de forma completamente diversa das condições de projeto consideradas, com mudanças arbitrárias feitas pelo cliente

Com a finalidade de familiarizar o leitor com os setores mais importantes de uma usina de açúcar e/ou alcool, apresenta-se a seguir uma descrição geral básica de cada um deles

- Alimentação de cana : compreende a chegada da cana com o caminhão, e sua descarga nos transportadores metálicos para a sua movimentação até o setor onde ocorre o processo de extração de caldo. No decorrer desse percurso a cana é lavada, depois picada e desfibrada para que se obtenha uma maior eficiência no processo de extração. 


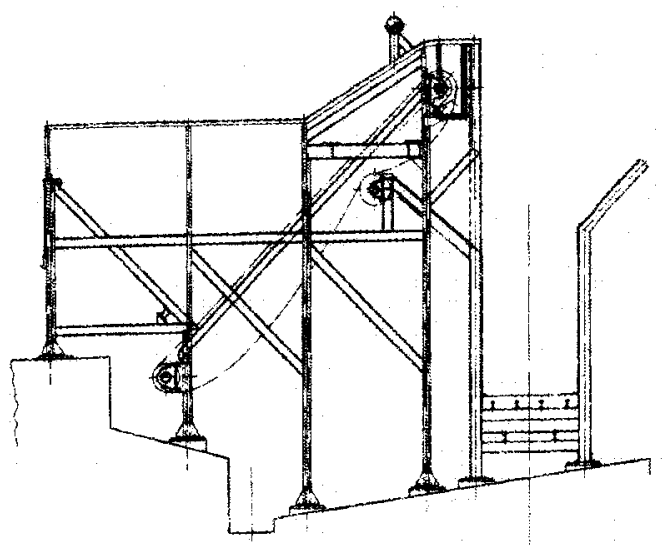

a) Mesa alimentadora

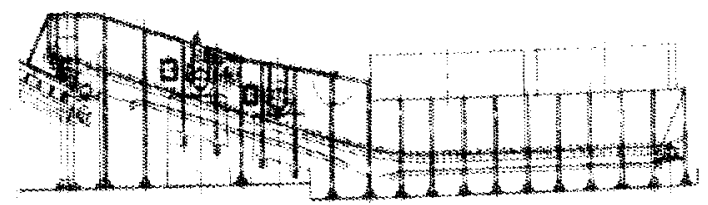

b) Esteira metálica de cana

FIGURA 1 - Equipamentos da alimentação de cana

- Extração de caldo : compreende a moagem da cana através de sua passagem por um conjunto de equipamentos que são chamados de "ternos" de moenda, dispostos em sequência, de forma a extrair o máximo de caldo possível da cana desfibrada.
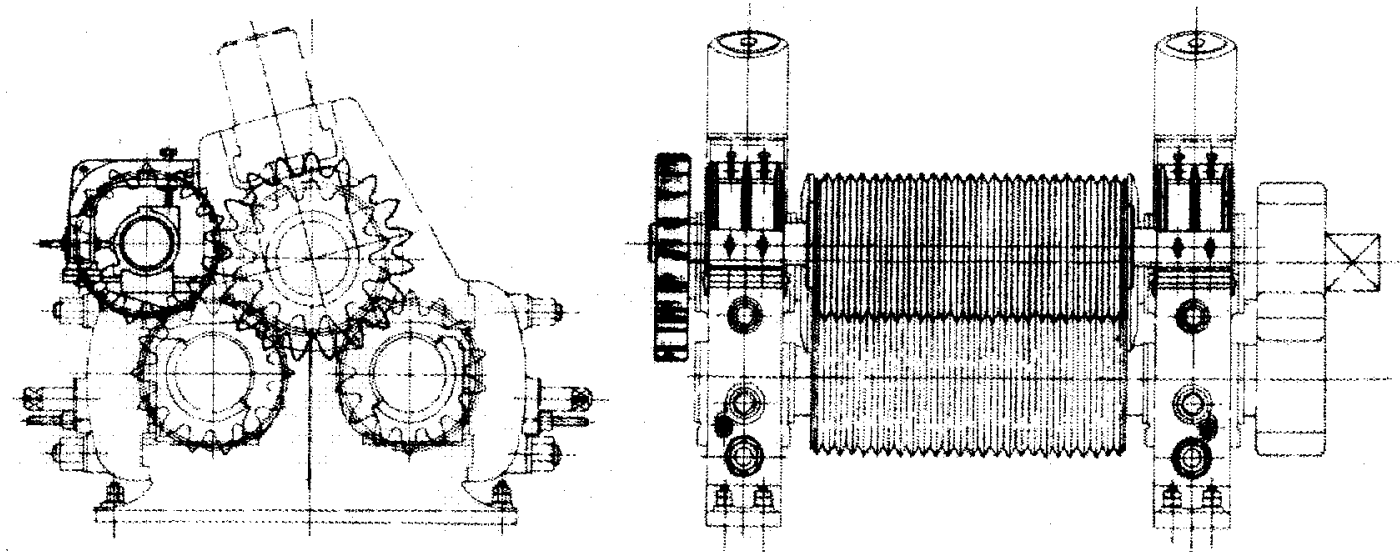

FIGURA 2 - Equipamento da extração de caldo : "terno" de moenda

- Movimentação e estocagem de bagaço : compreende um conjunto de transportadores metálicos e de correia existentes após a moagem, dispostos de forma a alimentarem, com o bagaço da cana, as caldeiras que o queimam para a geração da energia utilizada pela usina. A sobra de bagaço é estocada em um pátio para futuro consumo ou mesmo para comercialização. 

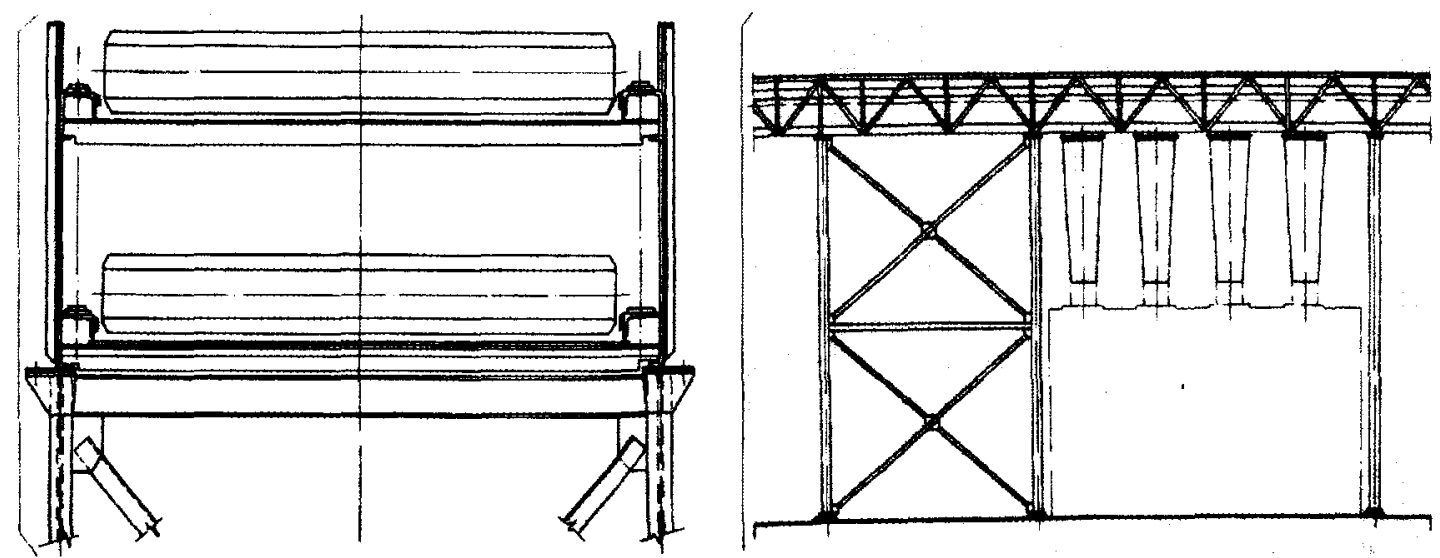

FIGURA 3 - Transportador metálico distribuidor de bagaço : seção transversal e vista lateral

- Fabricação de açúcar e alcool : compreende todos os equipamentos que são utilizados para os processos químicos de obtenção do açúcar e do alcool. Neste setor são encontrados : tanques para fermentação, evaporadores, cozedores, decantadores, equipamentos para a movimentação e estocagem de açúcar, tanques para estocagem de alcool, etc

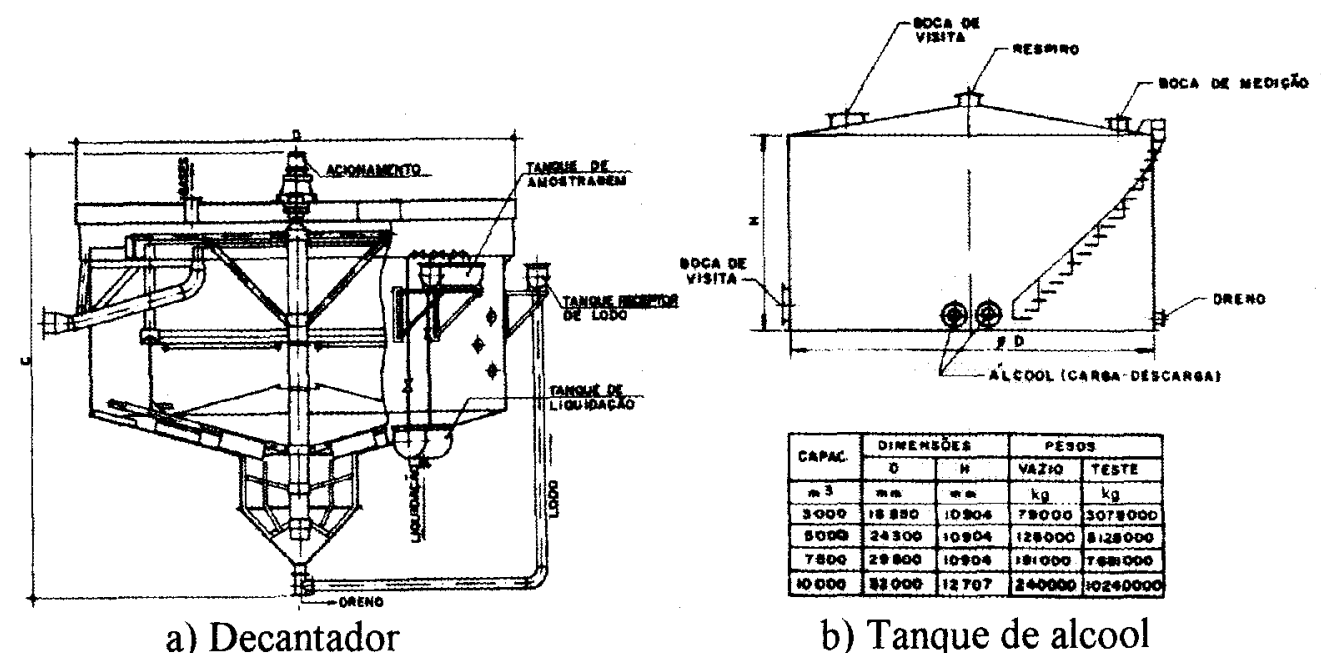

FIGURA 4 - Equipamentos da fabricação de açúcar e alcool

Este trabalho, visa auxiliar na análise estrutural de reservatórios metálicos elevados; equipamentos geralmente utilizados na área de processos da indústria sucroalcooleira, ou seja, no setor de fabricação de açúcar e alcool. 
Esses equipamentos são compostos por estruturas laminares não planas, as quais são chamadas de cascas. A maioria das superficies geometricamente definidas, utilizadas nas estruturas em casca desses equipamentos, são geradas a partir de uma curva girando ao redor de uma linha chamada "eixo de rotação", são as "superficies de revolução".

A superfície de revolução com eixo de rotação vertical e com a curva que a defini interceptando este eixo é denominada de "cúpula", sendo a curva de revolução chamada de "meridiano". O plano que a contém é chamado "plano meridiano da superfície", e as seções horizontais são chamadas de "paralelos".

Uma reta paralela ao eixo de rotação, usada como meridiano, gera uma "superfície cilíndrica". Uma reta inclinada em relação ao eixo de rotação interceptando o mesmo, usada como meridiano, gera uma "superficie cônica".

As superficies podem ser classificadas em categorias distintas, de acordo com a variação de sua curvatura em torno de um ponto. Cascas cilíndricas e cônicas são classificadas como cascas com "curvatura Gaussiana nula".

Nesse trabalho estudaremos o comportamento estrutural de cascas cilíndricas e cônicas, as quais, na liguagem usual das indústrias do setor sucro-alcooleiro, são conhecidas como "costado cilíndrico" e "fundo (por ser uma cúpula "invertida") cônico".

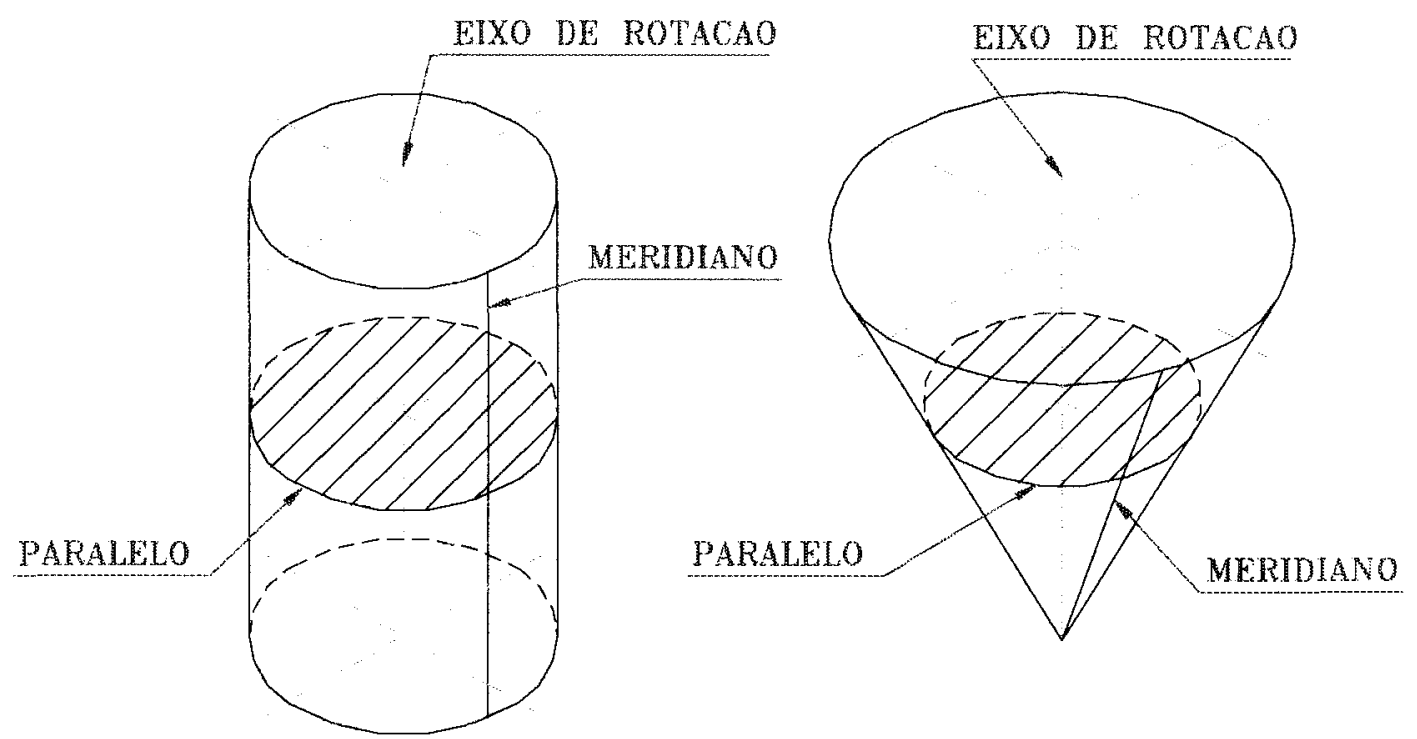

FIGURA 5 - Cascas cilíndrica e cônica 
Em 1989, participou-se do projeto de um equipamento bastante utilizado na área de processos da indústria de açucar e álcool, e descreve-se a seguir algumas características gerais deste típico reservatório metálico elevado, hipóteses de carregamento, critérios e comentários sobre as dificuldades encontradas na época.

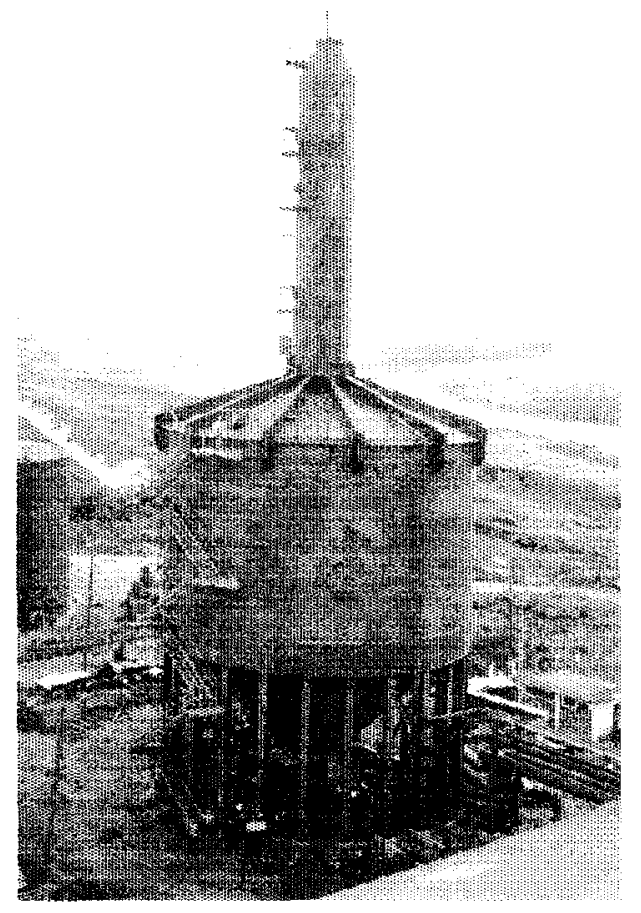

FIGURA 6 - Foto de dorna de fermentação de usina de açúcar na região de Catanduva - S.P.

- Características gerais:

- Designação : Dorna de fermentação

- Descrição : Reservatório metálico elevado onde ocorre o processo de fermentação alcoólica, formado por corpo cilíndrico, fundo e tampo cônicos, sustentado por estrutura metálica.

- Volume útil para fermentação $2000\left[\mathrm{~m}^{3}\right]$

- Diâmetro do corpo cilíndrico (costado) $1600[\mathrm{~cm}]$

- Altura do corpo cilíndrico (costado) $1200[\mathrm{~cm}]$

- Inclinação do fundo cônico com a horizontal $: 45$ [graus]

- Inclinação do tampo cônico com a horizontal $: 15$ [graus]

- Altura da estrutura de sustentação do reservatório : $950[\mathrm{~cm}]$ 
- Ambiente de trabalho

aberto ao tempo

- Estrutura:

- Corpo cilíndrico, fundo e tampo cônicos em aço ASTM A283C;

- Estrutura de sustentação metálica em aço ASTM A36 composta de Perfis Soldados e/ou Laminados

\section{- Hipóteses de Carregamento :}

Equipamento totalmente cheio de caldo de cana (peso específico 1,2 [ton $\left./ \mathrm{m}^{3}\right]$ ), sobrecarga de 0,5 [m.c.a.] (metros de coluna de água), ação do vento atuando segundo Norma Brasileira NBR 6123/1980 (na época).

\section{- Critérios :}

Para corpo, fundo e tampo cônicos utilizou-se a teoria das estruturas em casca de revolução admitindo-se comportamento de membrana, associada às recomendações do código ASME (American Society of Mechanical Engineer [1968a]) para vasos de pressão.

No código ASME além de encontrar toda uma formulação para o cálculo de vasos submetidos a pressão interna (vasos cilíndricos, tampos cônicos, tampos toricônicos, tampos torisféricos, etc), encontrou-se também recomendações para tensões admissíveis a tração para o aço utilizado na temperatura de trabalho do equipamento, tensões admissíveis para evitar a perda de estabilidade em cilindros submetidos a cargas de compressão axial, eficiência de solda, etc.

A estrutura de sustentação foi dimensionada utilizando a Norma Americana AISC [1980a].

\section{- Comentários:}

No dimensionamento do corpo e fundo da dorna de fermentação, encontrouse alguma dificuldade no cálculo dos esforços que aparecem na união entre o corpo cilíndrico e o fundo cônico visto que as teorias passam a ser bastante complicadas na sua aplicação prática. Nesta união é que se encontra o ponto crítico do dimensionamento com tensões bastante elevadas.

Para evitar a aplicação destas teorias mais complicadas, devido à necessidade de solução rápida e segura para o desenvolvimento do projeto, adotaram-se modelos estruturais que permitiam simplificar a análise da união cilindro-cone. 
Estas situações não ocorriam com os projetos antigos de dornas de fermentação e decantadores, que eram sustentadas por cascas cônicas de concreto, nas quais todo o fundo metálico era apoiado.

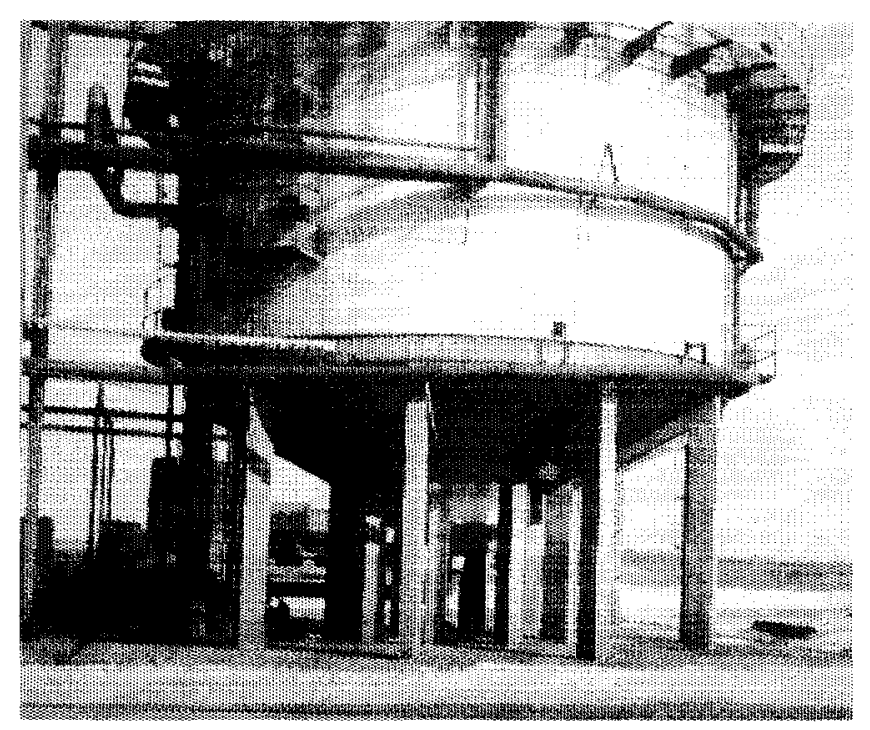

FIGURA 7 - Foto de decantador com fundo sustentado por casca cônica de concreto

O modelo estrutural utilizado consistiu no projeto de uma viga circular ("viga anel") tipo caixão na região onde ocorria a união cilindro-cone, apoiando sobre esta viga o corpo cilíndrico, e sustentando sob esta viga o fundo cônico, dimensionando a mesma para suportar os esforços de compressão, flexão e torção causados pelas reações do cilindro e do cone devidas a ação da carga de coluna de caldo nos mesmos. Com este modelo resolveu-se a viga circular, e também o corpo e o fundo.

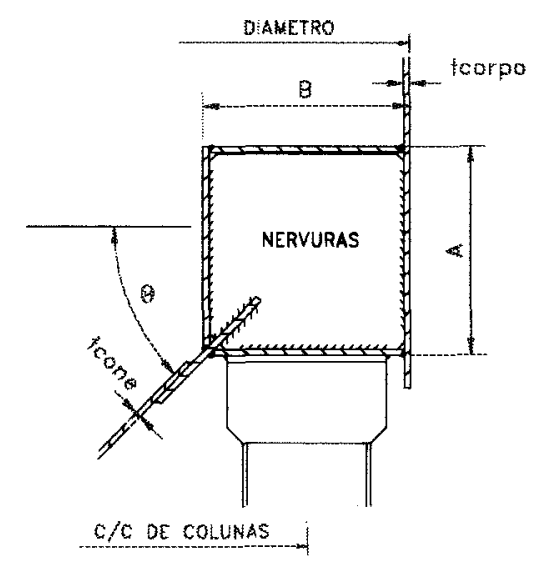

FIGURA 8 - Seção transversal da "viga anel" 
Outro problema encontrado foi na solução do fundo cônico, com ângulo de inclinação com a horizontal menor que 45 [graus]. O código ASME, cobre o dimensionamento de tampos cônicos com ângulos de inclinação com a horizontal maiores ou iguais a 60 [graus]. No outro extremo encontram-se algumas literaturas (BROWNELL [1959]) que sugerem que para cones submetidos a pressão externa com ângulos de inclinação com horizontal menores que 30 [graus], deve-se dimensionar o cone como uma placa plana carregada com carga uniformemente distribuída e bordos engastados.

Para não se correr o risco de trabalhar fora das recomendações do código ASME, os fundos cônicos com ângulos de inclinação com a horizontal menores que 60 [graus], eram muitas vezes projetados como placa reforçada por uma grelha circular/radial metálica, por ser modelo estrutural conhecido, mas não se considerava a resistência da estrutura cônica como casca de revolução, levando, em alguns casos, à espessuras super-dimensionadas do fundo

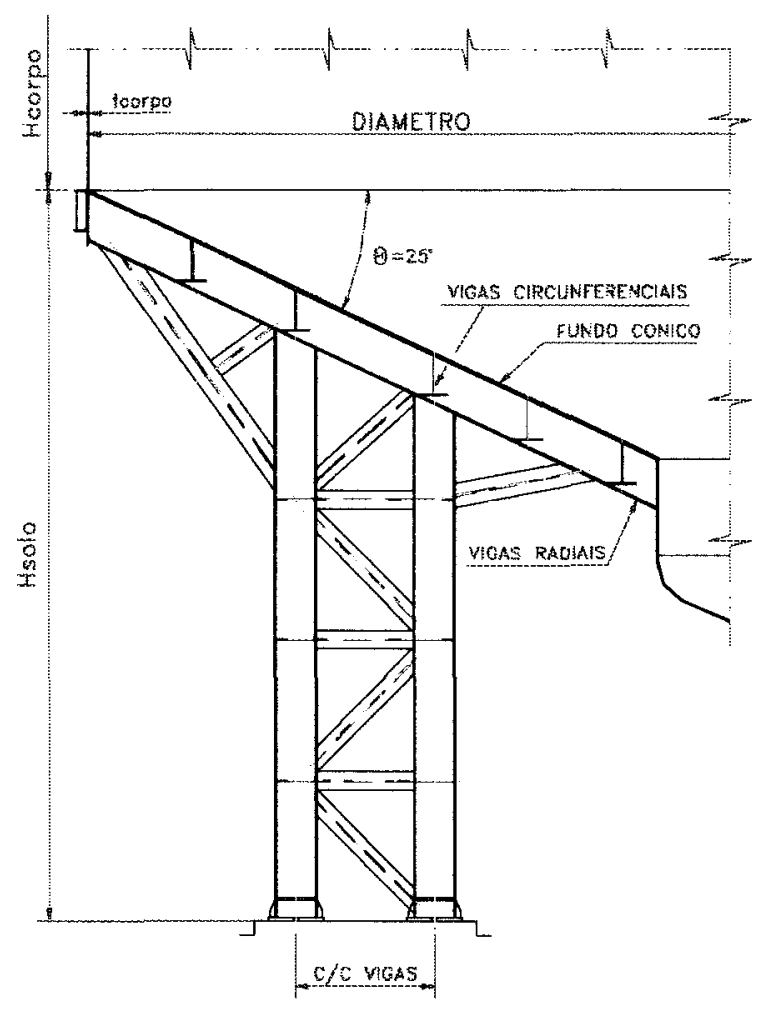

FIGURA 9 - Fundo cônico com reforço tipo "grelha circular/radial metálica" 
Após ter-se calculado alguns modelos de fundos cônicos em programa de análise de estruturas por elementos finitos (SAP90), notou-se que com ângulos de inclinação com a horizontal maiores ou iguais a 45 [graus], utilizando o modelo estrutural da viga circular, os mesmos poderiam ser dimensionados com relativa segurança pela teoria das estruturas de casca de revolução com comportamento de membrana.

Depois de projetado e construído o equipamento, decidiu-se fazer um ensaio para a medição das tensões atuantes, nas cascas cilíndrica e cônica, em campo, e contratou-se a equipe do Departamento de Engenharia Mecânica da Escola de Engenharia de São Carlos para o trabalho.

No acompanhamento do trabalho em campo observou-se a grande quantidade de imperfeições iniciais de fabricação no equipamento, e, os resultados dos ensaios puderam confirmar a impossibilidade de prever as tensões atuantes no corpo cilíndrico e no fundo cônico simplesmente com as análises teóricas, ou mesmo resolvendo os elementos estruturais que compunham o equipamento através de programa de análise de estruturas por elementos finitos.

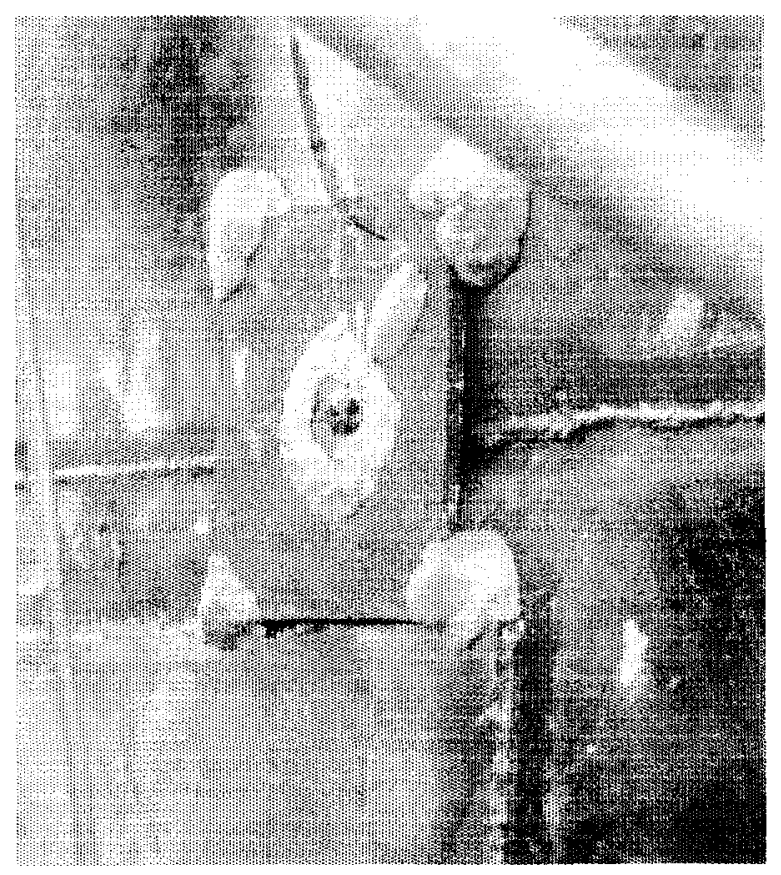

FIGURA 10 - Foto onde se pode visualisar imperfeição inicial no corpo cilíndrico 
Essa experiência pessoal vivenciada, tendo em vista os questionamentos por ela levantados, os quais se repetiram em vários outros projetos semelhantes, levou à crer que algo introdutório neste sentido, na linha de pesquisas de silos e reservatórios metálicos, seria de grande utilidade ao engenheiro do setor sucro-alcooleiro e aos profissionais da área de projetos estruturais em geral.

Este trabalho portanto, teve como objetivo, utilizar a teoria de estruturas em casca de revolução e programa "comercial" de análise de estruturas por elementos finitos, como ferramentas, no cálculo estrutural de cascas cilíndrica e cônica que compõem um equipamento com dimensões "típicas" utilizadas na área de processos da indústria sucro-alcooleira.

Obteve-se assim o equacionamento de expressões teóricas aproximadas mais acessíveis do que normalmente o são os programas de análise por elementos finitos a esse engenheiro de projetos.

Foram consideradas duas vinculações de borda : engastada e apoiada fixa.

Utilizou-se neste trabalho um sistema de unidades coerente com o cotidiano do profissional da indústria do açúcar e do alcool. 


\section{EQUACIONAMENTO DAS EXPRESSÕES TEÓRICAS}

\subsection{CONSIDERAÇÕES GERAIS}

Este trabalho, por ser destinado ao engenheiro de projetos que atua no setor sucro-alcooleiro, utilizou, no equacionamento das expressões teóricas, uma abordagem mais preocupada com a aplicação prática da teoria, do que com o seu desenvolvimento matemático propriamente dito.

Não pretendeu-se desenvolver um estudo profundo da teoria das cascas de revolução, mas sim aplicá-la como ferramenta prática no cálculo estrutural especificamente de cascas cilíndricas e cônicas.

No estudo das estruturas de superficie pode-se utilizar a teoria simplificada para cascas delgadas, desde que sejam obedecidas as seguintes hipóteses fundamentais de "Kirchoff-Love" :

a) o material que constitui a estrutura é homogêneo, isótropo e tem comportamento elástico linear (obedece a Lei de Hooke);

b) a espessura " $t$ " é pequena em relação às dimensões e aos raios de curvatura principais da superficie média (apresentam a relação do seu raio de curvatura pela sua espessura $\mathbf{R} / \mathbf{t} \geq 20$ );

c) as tensões normais à superfície média são desprezíveis em relação às demais tensões;

d) os pontos pertencentes, antes da deformação a retas normais à superficie média encontram-se, após a deformação, sobre retas perpendiculares à superficie média deformada (corresponde à hipótese de Navier da Resistência dos Materiais);

e) os deslocamentos são muitos pequenos em relação à espessura " $t$ " da casca, sendo possível desprezar a influência dos mesmos no estudo das condições de 
equilíbrio do elemento de superficie (teoria linear, sendo válida a superposição de efeitos)

Uma casca delgada submetida a ações externas desenvolve "tensões de membrana", isto é, tensões de tração, de compressão e cisalhamento.

Afim de poder desenvolver tensões de membrana sobre toda sua superficie, a casca delgada deverá ser ou estar corretamente vinculada, sendo que uma vinculação adequada é aquela que desenvolve também "reações de membrana", isto é, reações que atuam no plano tangente à casca, nas bordas vinculadas, e permite que as mesmas se desloquem devido aos esforços resultantes das tensões de membrana.

$\mathrm{Se}$ as reações na vinculação não forem tangentes à casca ou se os deslocamentos de membrana forem impedidos pela mesma, serão desenvolvidas também tensões provenientes da flexão, as quais se localizam nas vizinhanças das bordas vinculadas, ocasionando uma "perturbação de borda".

Uma casca cilíndrica de revolução simetricamente carregada, com vinculação contínua do tipo apoio articulado móvel em todo o contorno de sua borda inferior, apresentará comportamento análogo ao da membrana, desenvolvendo somente tensões normais de tração ou compressão e tensões tangenciais nulas, devido à simetria.

$\mathrm{Na}$ mesma casca anterior se for considerado apoio articulado fixo, o impedimento do deslocamento radial, gera uma reação horizontal radial distribuída ao longo do paralelo vinculado, a qual não sendo tangente à casca, gera momento fletor em torno da borda, criando por sua vez tensões de flexão.

Considerando-se um engastamento contínuo, estará sendo impedindo além do deslocamento radial da casca, a rotação ao longo do seu meridiano, gerando uma reação horizontal radial e um momento, distribuídos ao longo do paralelo vinculado, e novamente haverá o aparecimento de tensões de flexão.

Portanto, para que a casca se comporte como uma membrana, são necessários os seguintes requisitos de projeto e condições de carregamento :

a) a casca deverá ser delgada, de espessura constante, ou então a mesma deverá variar gradativamente, evitando-se variações bruscas; 
b) a casca deverá ter uma forma adequada, isto é, a superficie da mesma deve ser contínua e a curvatura deverá variar gradativamente;

c) a casca deverá estar submetida à forças distribuídas que variam contínua e suavemente, isto é, sem variações bruscas nas suas intensidades;

d) a casca deverá estar corretamente apoiada, de tal maneira que os esforços que atuam na borda da mesma, devam ser tangentes à superfície média; as deformações na borda devem acomodar-se ou acompanhar as deformações dos elementos contíguos.

As cascas cilíndrica e cônica pertencentes aos equipamentos normalmente utilizados na área de processos do setor sucro-alcooleiro, possuem as seguintes características :

a) o material das cascas é o aço carbono;

b) a relação $R / \mathbf{t}$ é muito maior que 20 ;

c) as tensões normais à superficie da casca são desprezíveis;

d) os deslocamentos podem ser considerados relativamente pequenos em relação à espessura da casca;

e) normalmente não são consideradas variações bruscas de espessura ao longo do meridiano da casca;

f) as cascas possuem superficies contínuas e curvaturas bem definidas, sendo simétricas em relação a um eixo de rotação;

g) as ações externas atuantes são geralmente carregamentos simétricos de pressão de gases e/ou pressão hidrostática do produto armazenado;

h) as vinculações nem sempre permitem o deslocamento livre do borda, assemelhando-se geralmente a apoios articulados fixos ou engastes, ambos contínuos.

Portanto, essas cascas, foram consideradas como obedecendo, de maneira relativamente aceitável em termos de engenharia, às hipóteses de "Kirchoff-Love", aos requisitos básicos de carregamento e à quase todos os requisitos básicos de projeto para que se comporte como uma membrana, excetuando-se às vinculações das bordas. 
No equacionamento das expressões teóricas, foi considerada a Teoria de Membrana para a análise do comportamento geral da casca, superpondo os efeitos de "perturbação de borda" causados pelos esforços originados pelas vinculações da mesma.

As tensões de flexão desenvolvidas nas vizinhanças das bordas vinculadas da casca, podem ter valores elevados, mas amortecem com rapidez, de modo oscilatório, a medida que distancia-se do vinculo.

BELLUZZI [1967] apresenta dois capítulos dedicados à solução de problemas de cascas de revolução. As suas soluções foram aplicadas na resolução das estruturas que fizeram parte deste trabalho.

Todo desenvolvimento estará apresentado como um "memorial de cálculo", permitindo a aplicação direta das equações encontradas na casca de revolução cilíndrica ou cônica que se deseja verificar.

\subsection{CASCA CILÍNDRICA}

Considerou-se uma casca cilíndrica de revolução, de espessura constante, sob ação de pressão hidrostática, vinculada continuamente na sua borda inferior primeiramente por um engastamento e depois por apoio articulado fixo.

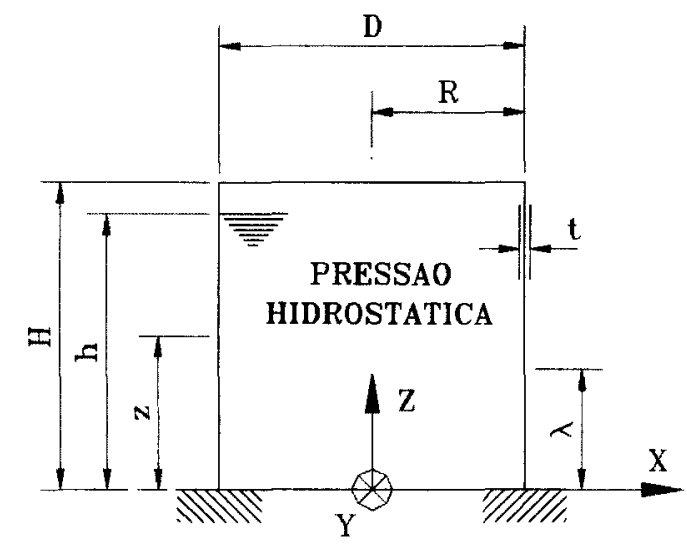

a) borda inferior engastada

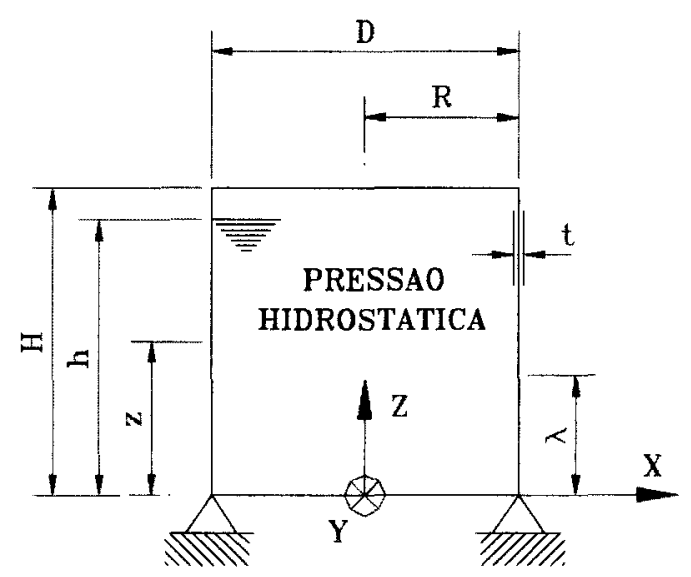

b) borda inferior apoiada fixa

FIGURA 11 - Casca cilíndrica de revolução sob ação de pressão hidrostática

Será chamado de " $\gamma$ " o peso específico do produto armazenado em [ton $/ \mathrm{cm}^{3}$ ], e as dimensões da casca cilíndrica serão 
D - diâmetro da superficie média da casca em [cm]

$\mathrm{R}$ - raio da superficie média da casca em [cm]

$\mathrm{H}$ - altura total da casca em [cm]

$\mathrm{h}$ - altura da coluna de produto armazenado em [cm]

$\mathrm{t}$ - espessura da casca em $[\mathrm{cm}]$

$\mathrm{Z}$ - eixo vertical medido a partir da borda vinculada coincidente com o eixo de rotação da casca

$\mathrm{X}$ - eixo horizontal pertencente ao plano do papel, medido a partir do início do eixo vertical " $Z$ "

Y - eixo horizontal que fura o plano do papel, medido a partir do início do eixo vertical "Z"

$\mathrm{z}$ - coordenada medida na direção do eixo " $\mathrm{Z}$ "

Conforme BELLUZZI, os coeficientes básicos para casca cilíndrica que serão utilizados no decorrer do equacionamento são :

$$
\beta=\frac{E \cdot t}{R^{2}} \quad \text { (1) } \quad B=\frac{E \cdot t^{3}}{12 \cdot\left(1-v^{2}\right)} \quad \text { (2) } \quad \alpha=\frac{\sqrt[4]{3 \cdot\left(1-v^{2}\right)}}{\sqrt{R \cdot t}}
$$

onde E é o modulo de elasticidade em [ton $/ \mathrm{cm}^{2}$ ] e $v$ é o coeficiente de Poisson para o material da casca.

O comprimento, ao longo do meridiano da casca, necessário para o amortecimento das tensões de flexão causadas pela "perturbação de borda" pode ser calculado a partir da solução da expressão geral que define o deslocamento radial da casca cilíndrica para o efeito provocado por forças radiais ou momentos distribuídos ao longo do paralelo vinculado.

Será chamado de " $\lambda$ " este comprimento, em [cm], sendo encontrado através da equação : 


$$
\lambda=\frac{2 . \pi}{\sqrt[4]{3 \cdot\left(1-v^{2}\right)}} \cdot \sqrt{\text { R.t }}
$$

Esse comprimento, medido a partir da borda vinculada, ajuda a definir se uma casca cilíndrica pode ser considerada como "longa" ou "curta". Para ser longa a altura da casca entre vinculações de borda deve ser maior ou igual ao valor de " $\lambda$ ". Na casca cilíndrica "longa" as condições de vinculação de uma das suas bordas praticamente não influencia no comportamento estrutural da outra borda, o que não ocorre na "curta". Estar-se-á trabalhando com casca cilíndrica "longa".

A dimensão " $\lambda$ " também permite saber a partir de que altura a casca tem seu comportamento representado, com boa precisão, pela Teoria de Membrana.

São chamados coeficientes elásticos de borda, o deslocamento radial e a rotação do meridiano da casca cilíndrica encontrados quando da aplicação de uma força radial externa unitária ou de um momento externo unitário, distribuídos uniformemente ao longo do perímetro da superficie média da borda inferior da mesma, e são descritos abaixo

- deslocamento radial da superficie média da borda inferior da casca cilindrica $\mathrm{em}\left[\mathrm{cm}^{2} /\right.$ ton $]$ para carga radial $\mathrm{f}=1$ ton $/ \mathrm{cm}$ :

$$
\text { wh }:=\frac{2 \cdot \alpha}{\beta}
$$

- deslocamento radial da superficie média da borda inferior da casca cilíndrica $\mathrm{em}[\mathrm{cm} /$ ton] para momento $\mathrm{m}=1$ ton $\mathrm{cm} / \mathrm{cm}$ :

$$
w m:=\frac{2 \cdot \alpha^{2}}{\beta}
$$

- rotação do meridiano da superfície média da borda inferior da casca cilindrica em $[\mathrm{cm} / \mathrm{ton}]$ para carga radial $\mathrm{f}=1$ ton $/ \mathrm{cm}$ : 


$$
\phi \mathrm{h}:=\frac{2 \cdot \alpha^{2}}{\beta}
$$

- rotação do meridiano da superficie média da borda inferior da casca cilíndrica em [ton $\left.{ }^{-1}\right]$ para momento $\mathrm{m}=1$ ton. $\mathrm{cm} / \mathrm{cm}$ :

$$
\phi \mathrm{m}:=\frac{4 \cdot \alpha^{3}}{\beta}
$$

No caso particular de uma casca cilíndrica sob a ação de pressão hidrostática de um líquido de peso específico " $\gamma$ " com sua borda inferior livre de qualquer vinculação que impeça seu deslocamento radial e rotação, encontra-se da Teoria de Membrana

- deslocamento radial para ação da coluna de líquido armazenado em [cm] :

$$
\mathrm{Wp}(\mathrm{z}):=\frac{\gamma \cdot \mathrm{R}^{2}}{\mathrm{E} \cdot \mathrm{t}} \cdot(\mathrm{h}-\mathrm{z})
$$

- rotação do meridiano para ação da coluna de líquido armazenado em [radianos] :

$$
\phi p(z):=\gamma \cdot \frac{R^{2}}{E \cdot t}
$$

Considerando-se as seguintes condições de contorno para borda inferior engastada (índice "e")

- deslocamento radial total e rotação total do meridiano nulos na coordenada $\mathrm{z}=0$ : 


$$
\begin{aligned}
& w p(z)+\text { Hce. } w h+\text { Mce. } w m=0 \\
& \phi p(z)+\text { Hce. } \phi h+\text { Mce. } \phi m=0
\end{aligned}
$$

Resolvendo o sistema de equações encontra-se a força e o momento necessários para garantirem o engastamento perfeito da borda inferior da casca :

- momento distribuído na borda engastada da casca em [ton $\mathrm{cm} / \mathrm{cm}]$ :

$$
\text { Mce }:=\gamma \cdot \frac{\alpha \cdot h-1}{2 \cdot \alpha^{3}}
$$

- força radial distribuída na borda engastada da casca em [ton $/ \mathrm{cm}]$ :

$$
\text { Hce }:=-\gamma \cdot \frac{2 \cdot \alpha \cdot h-1}{2 \cdot \alpha^{2}}
$$

Considerando-se agora as condições de contorno para borda inferior apoiada fixa (indice "a") :

- deslocamento radial total nulo na coordenada $\mathrm{z}=0$ :

$$
w p(z)+\text { Hca. } w h=0
$$

E resolvendo a equação encontra-se a força necessária para garantir o apoio fixo perfeito da borda inferior da casca :

- força radial distribuída na borda apoiada fixa da casca em [ton/ $/ \mathrm{cm}]$ :

$$
\text { Hca }:=-\gamma \cdot \frac{h}{2 \cdot \alpha}
$$




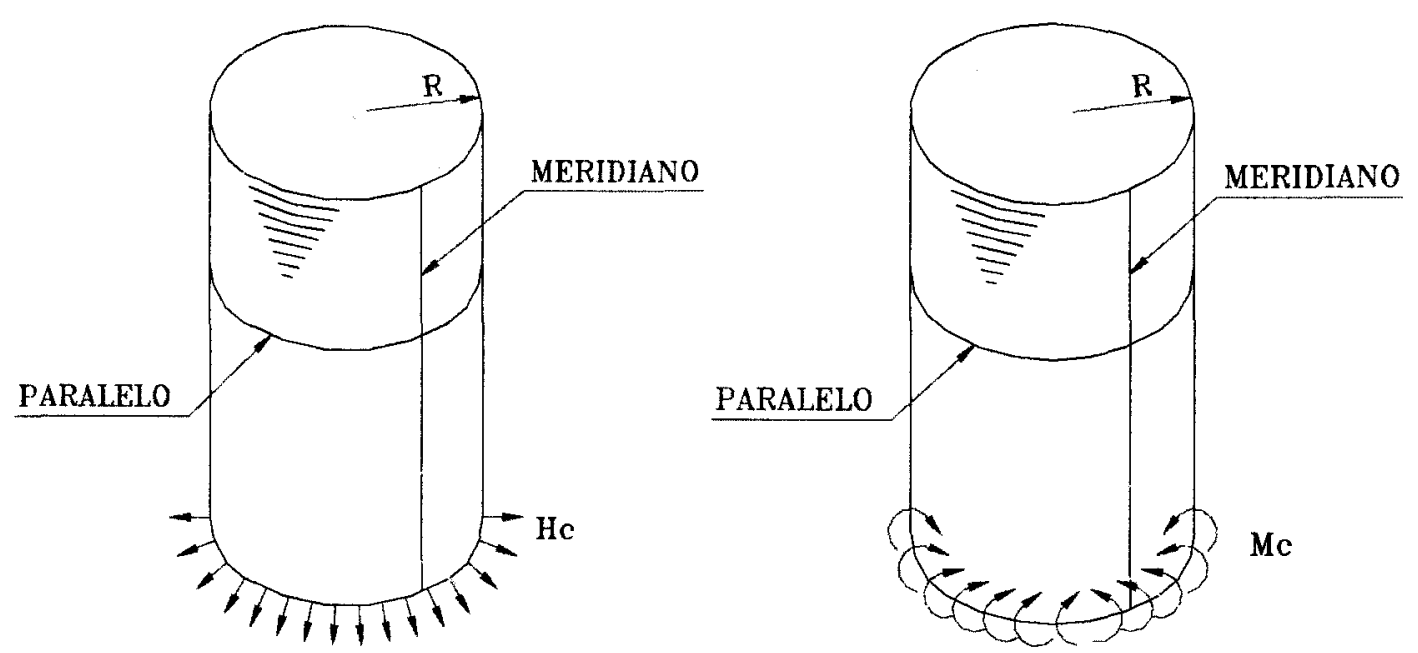

FIGURA 12 - Esquema para as forças e momentos distribuídos na borda vínculada

Através das equações da deformada dos meridianos da casca cilíndrica, desenvolvidas em BELLUZZI (cap.28) para diversas solicitações de borda, pode-se agora encontrar as equações para rotações, momentos e tensões através das seguintes expressões básicas da Teoria da Elasticidade

$$
\phi=-\frac{d W}{d z} \quad M 1=B \frac{d^{2} W}{d^{2}} \quad M 2=v M 1 \quad \sigma_{2}=\frac{E . t}{R} W
$$

\subsubsection{CASCA CHIINDRICA COM BORDA INFERIOR ENGASTADA}

Obtem-se então para borda inferior engastada (índice "e") :

- equação dos deslocamentos radiais totais ao longo do meridiano da superficie média da casca cilíndrica (força, momento e líquido armazenado) em $[\mathrm{cm}]$ :

$$
\begin{gathered}
W e(z)=\frac{\text { Hce }}{2 \cdot \alpha^{3} \cdot B} \cdot e^{-\alpha \cdot z} \cdot \sin \left(\alpha \cdot z+\frac{\pi}{2}\right)+\frac{\text { Mce } \cdot \sqrt{2}}{2 \cdot \alpha^{2} \cdot B} \cdot e^{-\alpha \cdot z} \cdot \sin \left(\alpha \cdot z+\frac{3 \pi}{4}\right)+ \\
+\frac{\gamma \cdot R^{2}}{\text { E.t }} \cdot(h-z)
\end{gathered}
$$


- equação das rotações totais ao longo do meridiano da superfície média da casca cilíndrica (força, momento e líquido armazenado) em [radianos] :

$$
\begin{gathered}
\phi e(z)=\frac{- \text { Hce }}{2 \cdot \alpha^{2} \cdot B} \cdot e^{-\alpha \cdot z} \cdot(\cos \alpha \cdot z+\sin \alpha \cdot z)+ \\
-\frac{\text { Mce } \cdot \sqrt{2}}{2 \cdot \alpha \cdot B} \cdot e^{-\alpha \cdot z} \cdot\left[\cos \left(\alpha \cdot z+\frac{\pi}{4}\right)+\sin \left(\alpha \cdot z+\frac{\pi}{4}\right)\right]-\frac{\gamma \cdot R^{2}}{E \cdot t}
\end{gathered}
$$

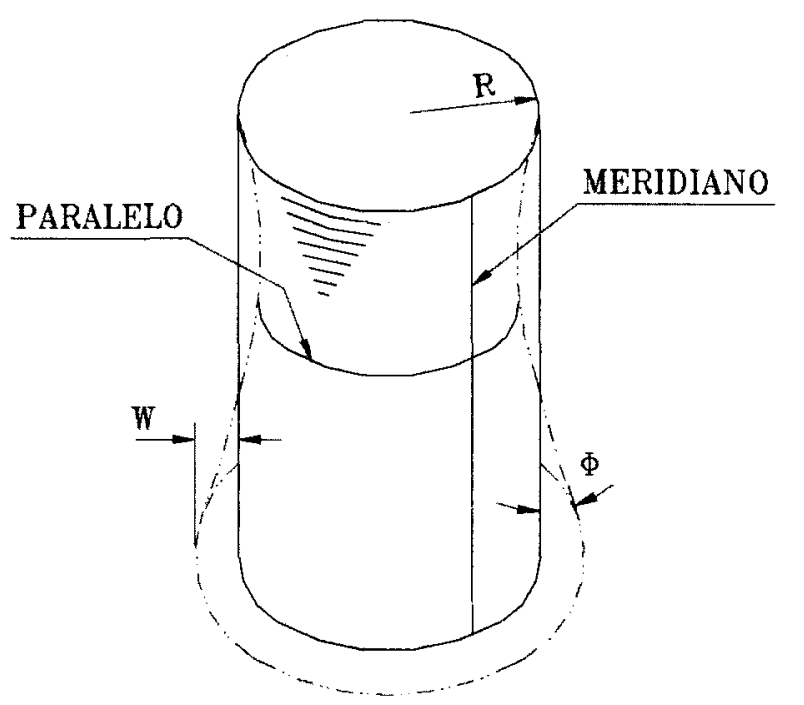

FIGURA 13 - Esquema para deslocamentos radiais e rotações

- equação dos momentos solicitantes ao longo dos paralelos da superfície média da casca cilíndrica para esforços distribuídos na borda engastada em [ton $\mathrm{cm} / \mathrm{cm}]$ :

$\operatorname{Mle}(z):=\frac{- \text { Hce }}{\alpha} \cdot \mathrm{e}^{-\alpha \cdot z} \cdot \sin (\alpha \cdot z)-\sqrt{2} \cdot M c e \cdot e^{-\alpha \cdot z} \cdot \sin \left(\alpha \cdot z+\frac{\pi}{4}\right)$

- equação dos momentos solicitantes ao longo dos meridianos da superfície média da casca cilíndrica para esforços distribuídos na borda engastada em [ton. $\mathrm{cm} / \mathrm{cm}$ ] : 


$$
\operatorname{M} 2 \mathrm{e}(\mathrm{z}):=v \cdot\left(\frac{- \text { Hce }}{\alpha} \cdot \mathrm{e}^{-\alpha \cdot \mathrm{z}} \cdot \sin (\alpha \cdot \mathrm{z})-\sqrt{2} \cdot \mathrm{Mce} \cdot \mathrm{e}^{-\alpha \cdot \mathrm{z}} \cdot \sin \left(\alpha \cdot \mathrm{z}+\frac{\pi}{4}\right)\right)
$$

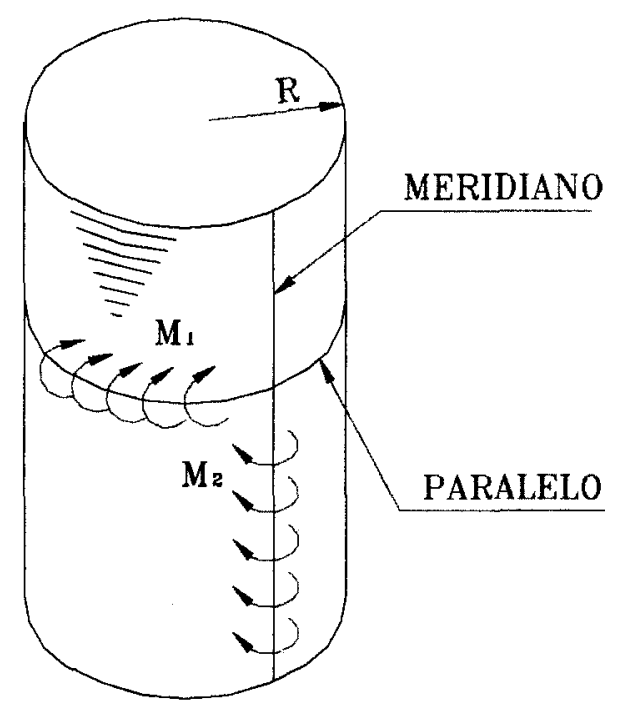

FIGURA 14 - Esquema para os momentos solicitantes

- equação das tensões ao longo dos meridianos das superficies interna (índice "in") e externa (índice "ex") da casca cilíndrica em $\left[\mathrm{ton} / \mathrm{cm}^{2}\right]$ :

$$
\begin{aligned}
& \sigma \operatorname{lein}(z):=\frac{-6 \cdot M 1 e(z)}{t^{2}} \\
& \sigma 1 \operatorname{eex}(z):=\frac{6 \cdot M 1 e(z)}{t^{2}}
\end{aligned}
$$

- equação das tensões ao longo dos paralelos das superficies interna (índice "in") e externa (índice "ex") da casca cilíndrica em [ton/ $\left.\mathrm{cm}^{2}\right]$ :

$$
\sigma 2 \operatorname{ein}(z):=\frac{E \cdot W e(z)}{R}-\frac{6 \cdot M 2 e(z)}{t^{2}}
$$




$$
\sigma 2 \operatorname{eex}(z):=\frac{E \cdot W e(z)}{R}+\frac{6 \cdot M 2 e(z)}{t^{2}}
$$

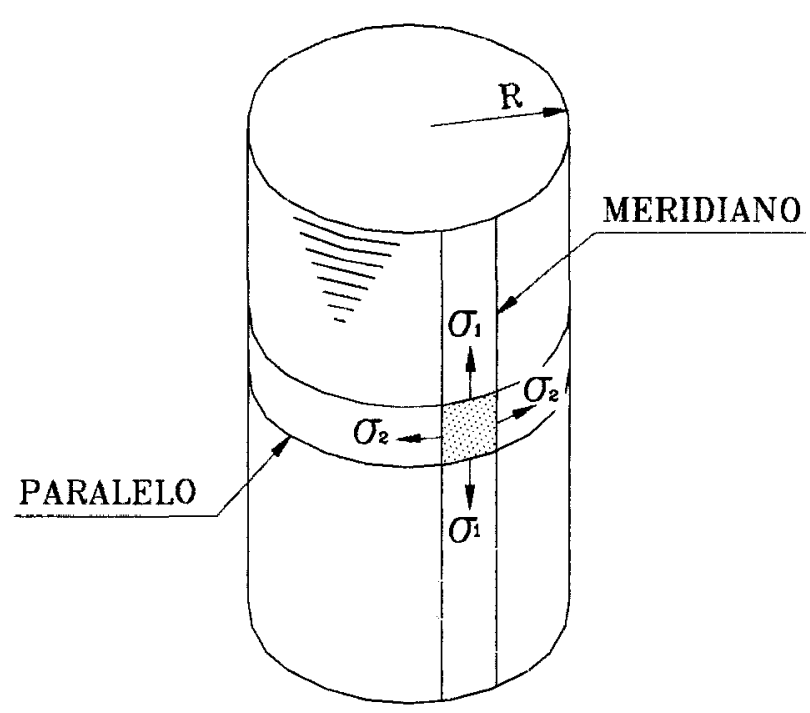

FIGURA 15 - Esquema para as tensões atuantes

\subsubsection{CASCA CILÍNDRICA COM BORDA INFERIOR APOIADA} FIXA

Para borda inferior apoiada fixa (índice "a"), encontrou-se então

- equação dos deslocamentos radiais totais ao longo do meridiano da superfície média da casca cilíndrica (força, momento e líquido armazenado) em $[\mathrm{cm}]$ :

$$
\mathrm{Wa}(\mathrm{z})=\frac{\mathrm{Hca}}{2 \cdot \alpha^{3} \cdot \mathrm{B}} \cdot \mathrm{e}^{-\alpha \cdot \mathrm{z}} \cdot \sin \left(\alpha \cdot \mathrm{z}+\frac{\pi}{2}\right)+\frac{\gamma \cdot \mathrm{R}^{2}}{\text { E. } \mathrm{t}} \cdot(\mathrm{h}-\mathrm{z})
$$

- equação das rotações totais ao longo do meridiano da superfície média da casca cilíndrica (força, momento e líquido armazenado) em [radianos] : 


$$
\phi a(z)=\frac{- \text { Hca }}{2 \cdot \alpha^{2} \cdot B} \cdot e^{-\alpha \cdot z} \cdot(\cos \alpha \cdot z+\sin \alpha \cdot z)-\frac{\gamma \cdot R^{2}}{E \cdot t}
$$

- equação dos momentos solicitantes ao longo dos paralelos da superficie média da casca cilíndrica para esforços distribuídos na borda apoiada fixa em [ton. $\mathrm{cm} / \mathrm{cm}$ ]

$$
\operatorname{Mla}(z):=\frac{- \text { Hca }}{\alpha} \cdot e^{-\alpha \cdot z} \cdot \sin (\alpha \cdot z)
$$

- equação dos momentos solicitantes ao longo dos meridianos da superficie média da casca cilíndrica para esforços distribuídos na borda apoiada fixa em [ton $\mathrm{cm} / \mathrm{cm}]$ :

$$
\mathrm{M} 2 \mathrm{a}(\mathrm{z}):=\frac{-v \cdot \mathrm{Hca}}{\alpha} \cdot \mathrm{e}^{-\alpha \cdot z} \cdot \sin (\alpha \cdot \mathrm{z})
$$

- equação das tensões ao longo dos meridianos das superfícies interna (índice "in") e externa (índice "ex") da casca cilíndrica em $\left[\mathrm{ton} / \mathrm{cm}^{2}\right]$ :

$$
\begin{aligned}
& \sigma \operatorname{lain}(z):=\frac{-6 \cdot M 1 a(z)}{t^{2}} \\
& \sigma 1 \operatorname{aex}(z):=\frac{6 \cdot M 1 a(z)}{t^{2}}
\end{aligned}
$$

- equação das tensões ao longo dos paralelos das superficies interna (índice "in") e externa (índice "ex") da casca cilíndrica em [ton/ $\left.\mathrm{cm}^{2}\right]$ :

$$
\sigma 2 \operatorname{ain}(z):=\frac{E \cdot W a(z)}{R}-\frac{6 \cdot M 2 a(z)}{t^{2}}
$$




$$
\sigma 2 \operatorname{aex}(z):=\frac{E \cdot W a(z)}{R}+\frac{6 \cdot M 2 a(z)}{t^{2}}
$$

\subsubsection{TENSÕES TEÓRICAS DE MEMBRANA}

As expressões das tensões teóricas de membrana para a casca cilíndrica sob ação da carga hidrostática do produto armazenado podem ser facilmente encontradas na resistência dos materiais, e estão aqui apresentadas para ilustração e comparação com as anteriormente encontradas (índice " $\mathrm{m} "$ ) :

- equação das tensões de membrana ao longo dos meridianos da superfície média da casca cilíndrica em $\left[\mathrm{ton} / \mathrm{cm}^{2}\right]$ :

A tensão de membrana ao longo dos meridianos é considerada nula visto que o atrito entre o líquido armazenado e a parede da casca cilíndrica é desprezível.

- equação das tensões de membrana ao longo dos paralelos da superfície média da casca cilíndrica em $\left[\mathrm{ton} / \mathrm{cm}^{2}\right]$ :

$$
\sigma 2 \mathrm{~m}(\mathrm{z}):=\frac{\gamma \cdot \mathrm{R}}{\mathrm{t}} \cdot(\mathrm{h}-\mathrm{z})
$$

E assim, através da utilização das equações de (1) a (32), pode-se resolver estruturalmente a maioria das cascas cilíndricas delgadas "longas", com borda inferior engastada ou apoiada fixa, submetidas a ação de pressão hidrostática, contanto que possuam características que atendam os comentários apresentados no item 2.1.

\subsection{CASCA CÔNICA}

Considerando agora, análogamente à casca cilíndrica, uma casca cônica de revolução, de espessura constante, sob ação de pressão hidrostática, vinculada continuamente na sua borda superior primeiramente por um engastamento e depois por apoio articulado fixo. 


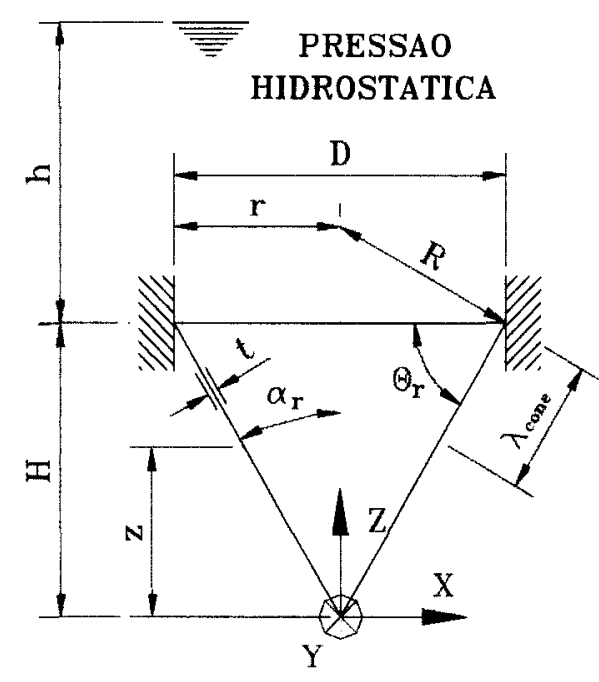

a) borda superior engastada

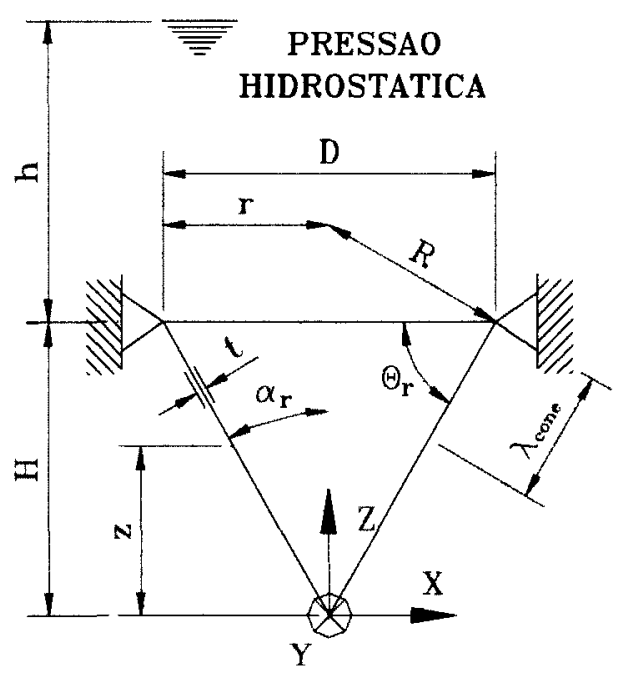

b) borda superior apoiada fixa

FIGURA 16 - Casca cônica de revolução sob ação de pressão hidrostática

Sendo " $\gamma$ " o peso específico do produto armazenado em [ton $\left./ \mathrm{cm}^{3}\right]$, e as dimensões da casca cônica como :

$\alpha r$ - ângulo de metade do bico da casca cônica com a vertical em [graus]

$\theta r=(90-\alpha r)$ - ângulo de metade do bico da casca cônica com a horizontal em [graus]

D - diâmetro da superficie média da borda superior da casca cônica em [cm]

$r=\frac{D}{2}-$ raio da superfície média da borda superior da casca cônica em [cm]

$\mathrm{R}=\frac{\mathrm{D}}{2 \cdot \cos (\alpha \mathrm{r})}-$ raio projetado da superficie média da borda superior da

casca cônica em $[\mathrm{cm}]$

$\mathrm{H}=\frac{\mathrm{D}}{2 \cdot \tan (\alpha \mathrm{r})}$ - altura total da casca cônica em $[\mathrm{cm}]$

h - altura da coluna de produto armazenado acima da casca cônica em [cm]

$\mathrm{t}$ - espessura da casca cônica em [cm]

$\mathrm{Z}$ - eixo vertical medido a partir do bico da casca cônica coincidente com o seu eixo de rotação

$\mathrm{X}$ - eixo horizontal pertencente ao plano do papel, medido a partir do início do eixo vertical "Z" 
Y - eixo horizontal que fura o plano do papel, medido a partir do início do eixo vertical " $\mathrm{Z}$ "

$\mathrm{z}$ - coordenada medida na direção do eixo " $\mathrm{Z}$ "

Os coeficientes básicos a serem utilizados no equacionamento da casca cônica, serão semelhantes aos da casca cilíndrica das expressões 1, 2, e 3 do item 2.2, utilizando-se $R=\frac{D}{2 \cdot \cos (\alpha r)}$

Recordando as equações (1), (2) e (3)

$$
\beta=\frac{E . t}{R^{2}} \quad B=\frac{E \cdot t^{3}}{12 \cdot\left(1-v^{2}\right)} \quad \alpha=\frac{\sqrt[4]{3 \cdot\left(1-v^{2}\right)}}{\sqrt{R \cdot t}}
$$

onde E é o modulo de elasticidade em [ton $\left./ \mathrm{cm}^{2}\right]$ e $v$ é o coeficiente de Poisson para o material da casca.

BELLUZZI orienta para uma solução aproximada de cascas esféricas não muito rebaixadas. Segundo ele, a aproximação é tanto melhor quanto mais rápido é o amortecimento das "perturbações de borda" ao longo da casca, ou seja, quanto maiores forem a relação $\mathbf{R} / \mathbf{t}$ e os ângulos da casca com a horizontal. Comenta que os resultados da solução aproximada são aceitáveis para ângulos $\theta \mathrm{r}$ maiores que 20 [graus]; e no caso da relação $\mathbf{R} / \mathbf{t}$ ser muito grande, também para ângulos $\theta \mathrm{r}$ não menores que 10 [graus].

BELLUZZI ainda afirma que, para qualquer casca de revolução (esférica, parabólica, elíptica, cônica, cilíndrica, etc.) de espessura constante, os coeficientes elásticos de borda podem ser obtidos através das equações da solução aproximada para cascas esféricas, transformando-se a casca que se pretende estudar em uma casca esférica equivalente. 
Fazendo-se então a utilização deste artificio para a casca cônica, foram desenvolvidas, como na casca cilíndrica, todas as equações necessárias para o cálculo estrutural de cascas cônicas com sua borda superior vinculada continuamente.

O comprimento, ao longo do meridiano da casca, necessário para o amortecimento das tensões de flexão causadas pela "perturbação de borda", pode também ser calculado a partir da solução da expressão geral que define o deslocamento radial da casca esférica equivalente a casca cônica estudada para o efeito provocado por forças radiais ou momentos distribuídos ao longo do paralelo vinculado

Sendo " $\lambda_{\text {cone }}$ este comprimento, em [cm], encontrado pela equação

$$
\lambda \text { cone }=R \cdot \tan \left[\frac{360}{\sqrt[4]{3 \cdot\left(1-v^{2}\right)}} \cdot \sqrt{\frac{t}{R}}\right]
$$

Medido a partir da borda vinculada tem função equivalente ao comprimento " $\lambda$ " na casca cilíndrica, sendo que o mais importante para o projeto, é que permite saber a partir de que distância da borda vinculada a casca cônica tem seu comportamento representado, com boa precisão, pela Teoria de Membrana.

Os coeficientes elásticos de borda, ou seja, o deslocamento radial e a rotação do meridiano da casca cônica encontrados quando da aplicação de uma força radial externa unitária ou de um momento externo unitário, distribuídos uniformemente ao longo do perímetro da superfície média da borda superior da mesma, são descritos abaixo

- deslocamento radial da superficie média da borda superior da casca cônica $\mathrm{em}\left[\mathrm{cm}^{2} /\right.$ ton $]$ para carga radial $\mathrm{f}=1$ ton $/ \mathrm{cm}$

$$
\xi \mathrm{h}:=\frac{2 \cdot \alpha}{\beta} \cdot \cos (\alpha \mathrm{r})^{2}
$$


- deslocamento radial da superficie média da borda superior da casca cônica $\mathrm{em}[\mathrm{cm} /$ ton] para momento $\mathrm{m}=1$ ton $. \mathrm{cm} / \mathrm{cm}$ :

$$
\xi \mathrm{m}:=\frac{2 \cdot \alpha^{2}}{\beta} \cdot \cos (\alpha \mathrm{r})
$$

- rotação do meridiano da superfície média da borda superior da casca cônica em $[\mathrm{cm} /$ ton] para carga radial $\mathrm{f}=1$ ton $/ \mathrm{cm}$ :

$$
\phi \mathrm{h}:=\frac{2 \cdot \alpha^{2}}{\beta} \cdot \cos (\alpha \mathbf{r})
$$

- rotação do meridiano da superfície média da borda superior da casca cônica em $\left[\right.$ ton $\left.^{-1}\right]$ para momento $\mathrm{m}=1$ ton $\mathrm{cm} / \mathrm{cm}$ :

$$
\phi m:=\frac{4 \cdot \alpha^{3}}{\beta}
$$

No caso particular de uma casca cônica sob a ação de pressão hidrostática de um líquido de peso específico " $\gamma$ " com sua borda superior livre de qualquer vinculação que impeça seu deslocamento radial e rotação, encontra-se da Teoria de Membrana :

- deslocamento radial para ação da coluna de líquido armazenado em [cm] :

$$
\xi p(z):=\frac{\gamma \cdot \tan (\alpha r)^{2} \cdot z^{2}}{E \cdot t \cdot \cos (\alpha r)} \cdot\left[(h+H-z)-\frac{v}{2} \cdot\left(h+H-\frac{2}{3} \cdot z\right)\right]
$$


- rotação do meridiano para ação da coluna de líquido armazenado em [radianos] :

$$
\phi p(z):=\frac{-\gamma \cdot \tan (\alpha r)^{2} \cdot z}{6 \cdot E \cdot t \cdot \cos (\alpha r)} \cdot(-9 \cdot h-9 \cdot H+16 \cdot z)
$$

Considerando-se as seguintes condições de contorno para borda superior da casca cônica engastada (índice "e") :

- deslocamento radial total e rotação total do meridiano nulos na coordenada $\mathrm{z}=\mathrm{H}$ :

$$
\begin{aligned}
& \xi \mathrm{p}(\mathrm{z})+\text { Hce. } \xi \mathrm{h}+\text { Mce. } \xi \mathrm{m}=0 \\
& \phi \mathrm{p}(\mathrm{z})+\text { Hce. } \phi \mathrm{h}+\text { Mce. } \phi \mathrm{m}=0
\end{aligned}
$$

Resolvendo o sistema de equações encontra-se a força e o momento necessários para garantirem o engastamento perfeito da borda superior da casca :

- momento distribuído na borda engastada da casca em [ton. $\mathrm{cm} / \mathrm{cm}]$ :

$$
\text { Mce }:=\frac{\xi \mathrm{p}(\mathrm{H})}{\xi \mathrm{m}}-\frac{2 \cdot \phi \mathrm{p}(\mathrm{H})}{\phi \mathrm{m}}
$$

- força radial distribuída na borda engastada da casca em [ton $/ \mathrm{cm}$ ]

$$
\text { Hce }:=\frac{\phi p(H)}{\phi h}-\frac{2 \cdot \xi p(H)}{\xi h}
$$

Considerando-se agora as condições de contorno para borda superior da casca cônica apoiada fixa (índice "a") : 
- deslocamento radial total nulo na coordenada $\mathrm{z}=\mathrm{H}$ :

$$
\xi \mathrm{p}(\mathrm{z})+\mathrm{Hca} . \xi \mathrm{h}=0
$$

E resolvendo a equação encontra-se a força necessária para garantir o apoio fixo perfeito da borda superior da casca

- força radial distribuída na borda apoiada fixa da casca em [ton $/ \mathrm{cm}]$

$$
\text { Hca }:=\frac{-\xi p(H)}{\xi h}
$$
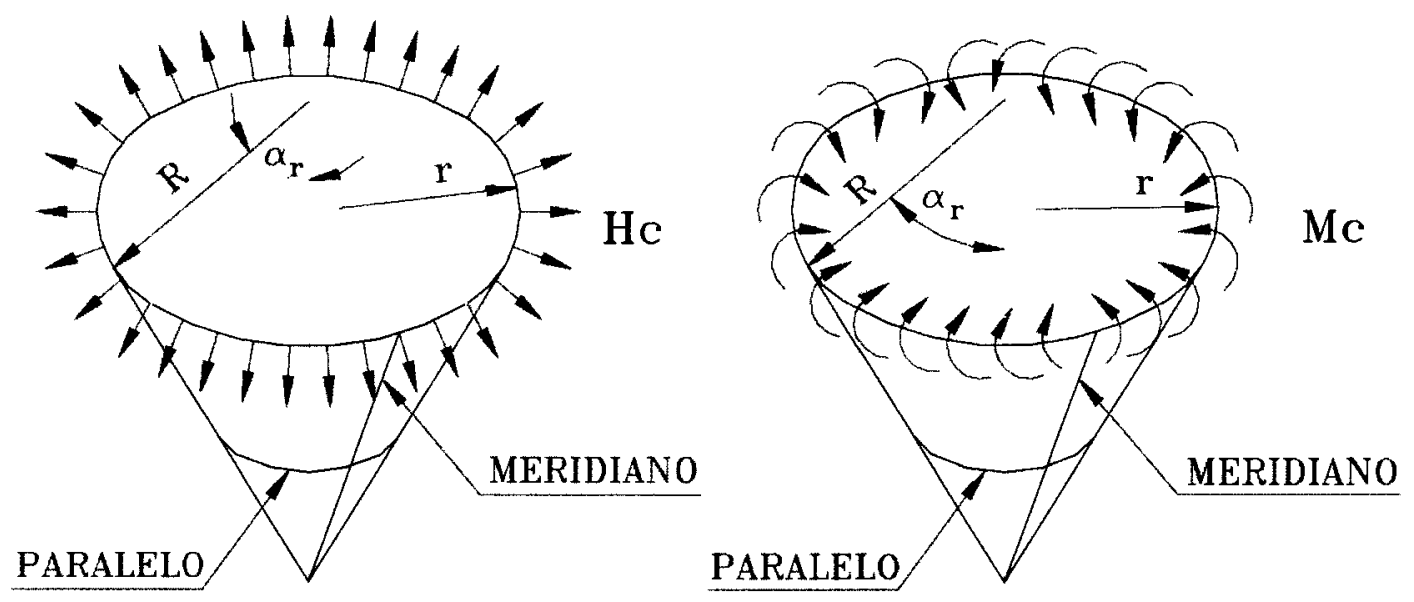

FIGURA 17 - Esquema para as forças e momentos distribuídos na borda superior vínculada

Através das equações da solução aproximada para cascas esféricas apresentadas em BELLUZZI, transformando-se a casca cônica em uma casca esférica equivalente e para diversas solicitações de borda, pode-se agora encontrar as equações para rotações, momentos e tensões através das expressões apresentadas abaixo. 


\subsubsection{CASCA CÔNICA COM BORDA SUPERIOR ENGASTADA}

Obtem-se então para borda superior engastada (índice "e")

- equação dos deslocamentos radiais totais ao longo do meridiano da superficie média da casca cônica (força, momento e líquido armazenado) em $[\mathrm{cm}]$ :

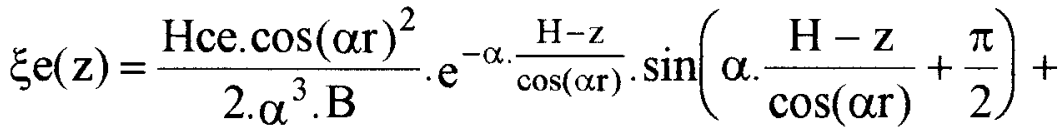

$$
\begin{aligned}
& +\frac{\text { Mce. } \cos (\alpha r) \cdot \sqrt{2}}{2 \cdot \alpha^{2} \cdot B} \cdot e^{-\alpha \cdot \frac{H-z}{\cos (\alpha r)}} \cdot \sin \left(\alpha \cdot \frac{H-z}{\cos (\alpha r)}+\frac{3 \cdot \pi}{4}\right)+ \\
& +\frac{\gamma \cdot \tan (\alpha r)^{2} \cdot z^{2}}{\text { E.t. } \cos (\alpha r)} \cdot\left[(h+H-z)-\frac{v}{2} \cdot\left(h+H-\frac{2}{3} \cdot z\right)\right]
\end{aligned}
$$

- equação das rotações totais ao longo do meridiano da superficie média da casca cônica (força, momento e líquido armazenado) em [radianos]

$$
\begin{aligned}
& \phi e(z)=\frac{H \operatorname{ce} \cdot \cos (\alpha r)}{2 \cdot \alpha^{2} \cdot B} \cdot e^{-\alpha \cdot \frac{H-z}{\cos (\alpha r)}} \cdot\left[\cos \left(\alpha \cdot \frac{H-z}{\cos (\alpha r)}\right)+\sin \left(\alpha \cdot \frac{H-z}{\cos (\alpha r)}\right)\right]+ \\
& +\frac{\text { Mce } \cdot \sqrt{2}}{2 \cdot \alpha \cdot B} \cdot e^{-\alpha \cdot \frac{H-z}{\cos (\alpha r)}} \cdot\left[\cos \left(\alpha \cdot \frac{H-z}{\cos (\alpha r)}+\frac{\pi}{4}\right)+\sin \left(\alpha \cdot \frac{H-z}{\cos (\alpha r)}+\frac{\pi}{4}\right)\right]+ \\
& -\frac{\gamma \cdot \tan (\alpha r)^{2} \cdot z}{6 \cdot \text { E.t. } \cos (\alpha r)} \cdot(-9 \cdot h-9 \cdot H+16 . z)
\end{aligned}
$$




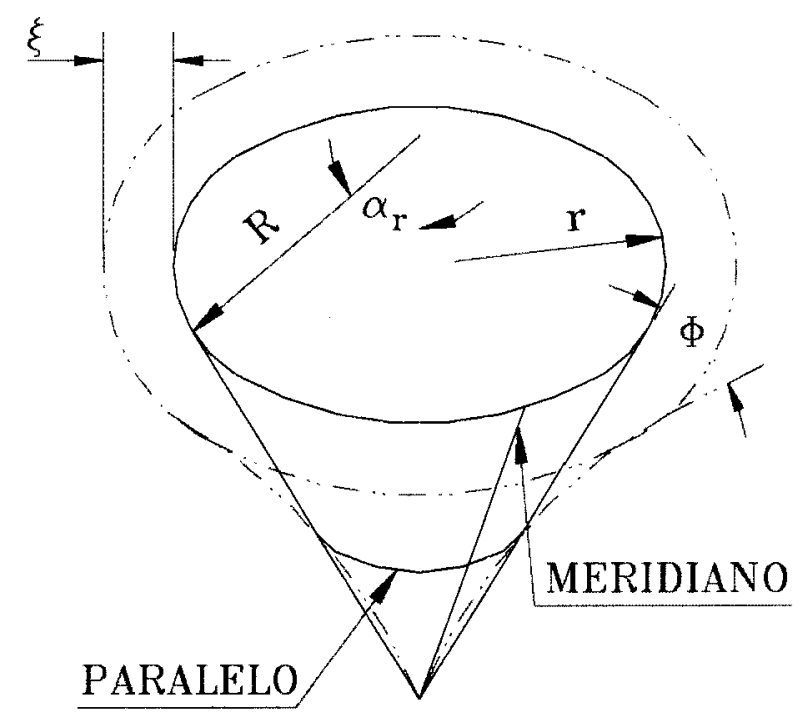

FIGURA 18 - Esquema para deslocamentos radiais e rotações

- equação dos momentos solicitantes ao longo dos paralelos da superfície média da casca cônica para esforços distribuídos na borda superior engastada $\mathrm{em}[$ ton $. \mathrm{cm} / \mathrm{cm}]$ :

$$
\begin{aligned}
& \operatorname{Mle}(z)=\frac{\text { Hce } \cdot \cos (\alpha r)}{\alpha} \cdot e^{-\alpha \cdot \frac{H-z}{\cos (\alpha r)} \cdot \sin \left(\alpha \cdot \frac{H-z}{\cos (\alpha r)}\right)+} \\
& +\sqrt{2} \cdot \text { Mce. } e^{-\alpha \cdot \frac{H-z}{\cos (\alpha r)}} \cdot \sin \left(\alpha \cdot \frac{H-z}{\cos (\alpha r)}+\frac{\pi}{4}\right)
\end{aligned}
$$

- equação dos momentos solicitantes ao longo dos meridianos da superfície média da casca cônica para esforços distribuídos na borda superior engastada $\mathrm{em}[$ ton. $\mathrm{cm} / \mathrm{cm}]$ :

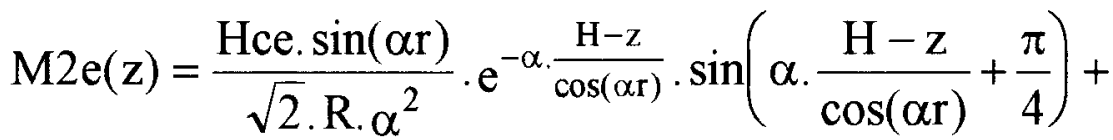

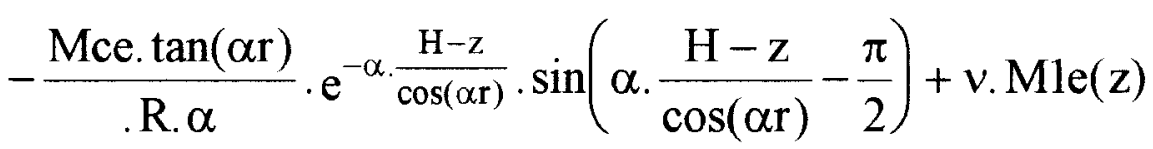




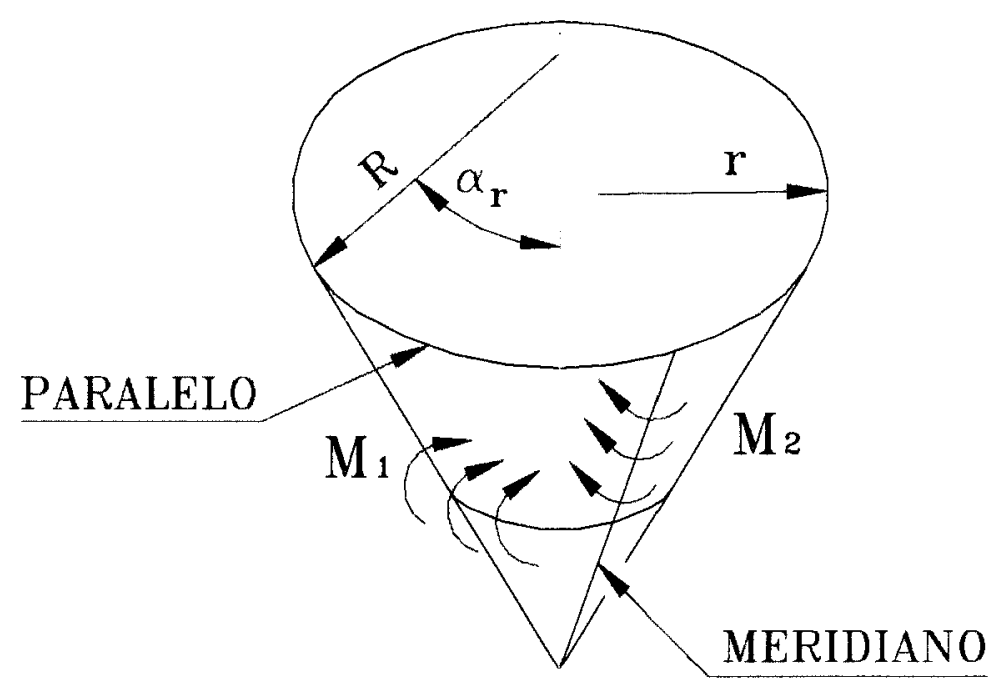

FIGURA 19 - Esquema para os momentos solicitantes

- equação das forças solicitantes ao longo dos meridianos da superficie média da casca cônica para esforços distribuídos na borda superior engastada em $[$ ton $/ \mathrm{cm}]$ :

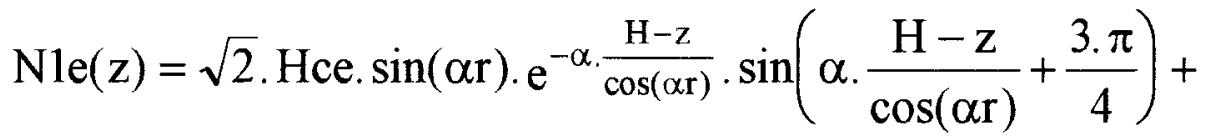

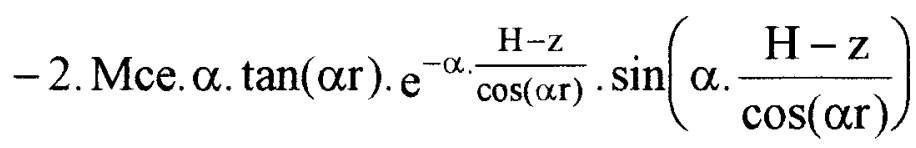

- equação das forças solicitantes ao longo dos paralelos da superfície média da casca cônica para esforços distribuídos na borda engastada em [ton $/ \mathrm{cm}]$ :

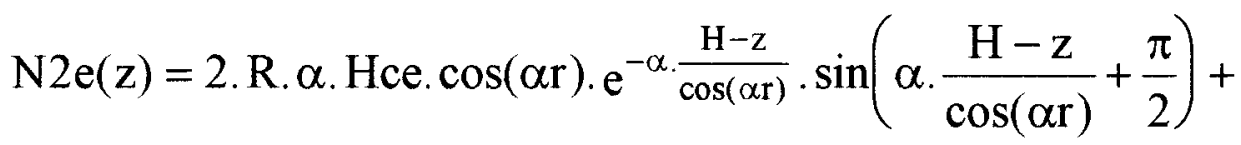

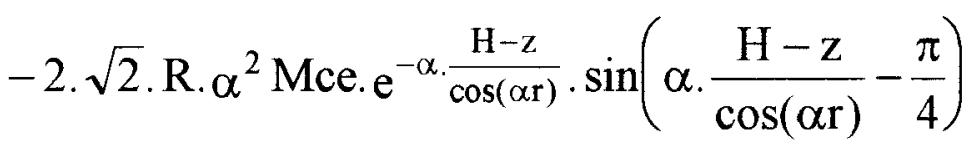




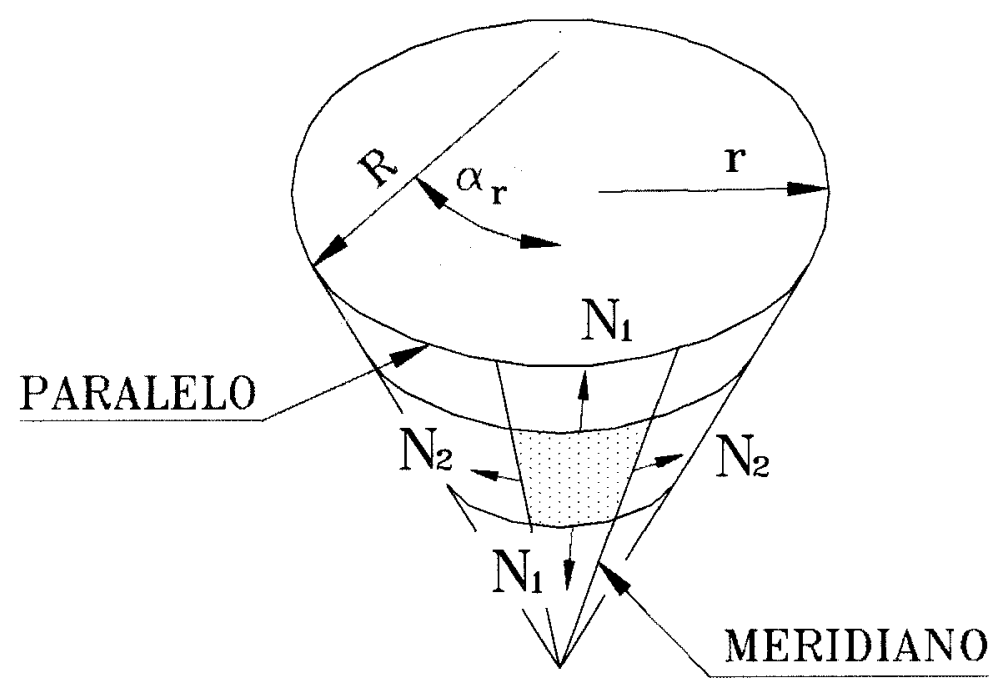

FIGURA 20 - Esquema para as forças solicitantes

- equação das tensões ao longo dos meridianos das superfícies interna (índice "in") e externa (índice "ex") da casca cônica em [ton $\left./ \mathrm{cm}^{2}\right]$ :

$$
\begin{aligned}
& \sigma 1 \operatorname{ein}(z):=\frac{\gamma \cdot \tan (\alpha r) \cdot z}{2 \cdot t \cdot \cos (\alpha r)} \cdot\left(h+H-\frac{2}{3} \cdot z\right)+\frac{N 1 e(z)}{t}-\frac{6 \cdot M 1 e(z)}{t^{2}} \\
& \sigma l \operatorname{eex}(z):=\frac{\gamma \cdot \tan (\alpha r) \cdot z}{2 \cdot t \cdot \cos (\alpha r)} \cdot\left(h+H-\frac{2}{3} \cdot z\right)+\frac{N 1 e(z)}{t}+\frac{6 \cdot M 1 e(z)}{t^{2}}
\end{aligned}
$$

- equação das tensões ao longo dos paralelos das superfícies interna (índice "in") e externa (índice "ex") da casca cônica em $\left[\operatorname{ton} / \mathrm{cm}^{2}\right]$ :

$$
\begin{aligned}
& \sigma 2 \operatorname{ein}(z):=\frac{\gamma \cdot \tan (\alpha r) \cdot z}{t \cdot \cos (\alpha r)} \cdot(h+H-z)+\frac{N 2 e(z)}{t}-\frac{6 \cdot M 2 e(z)}{t^{2}} \\
& \sigma 2 \operatorname{eex}(z):=\frac{\gamma \cdot \tan (\alpha r) \cdot z}{t \cdot \cos (\alpha r)} \cdot(h+H-z)+\frac{N 2 e(z)}{t}+\frac{6 \cdot M 2 e(z)}{t^{2}}
\end{aligned}
$$




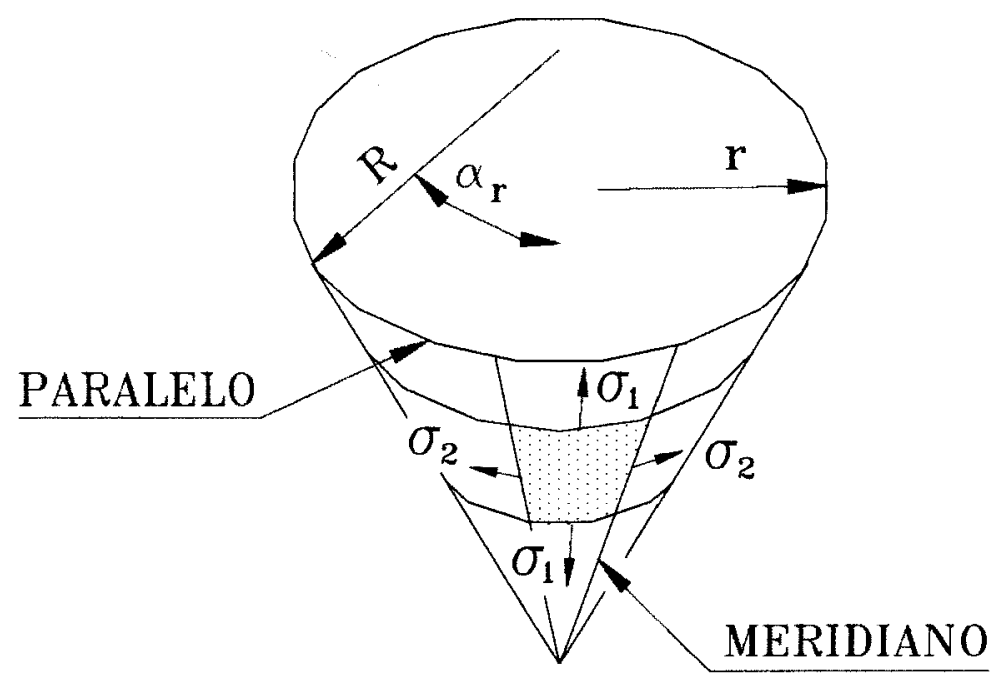

FIGURA 21 - Esquema para as tensões atuantes

\subsubsection{CASCA CÔNICA COM BORDA SUPERIOR APOIADA FIXA}

Para borda superior apoiada fixa (índice "a"), encontrou-se então :

- equação dos deslocamentos radiais totais ao longo do meridiano da superficie média da casca cônica (força, momento e líquido armazenado) em [cm]

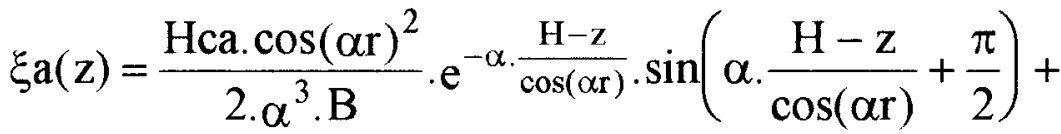

$$
\begin{aligned}
& +\frac{\gamma \cdot \tan (\alpha r)^{2} \cdot z^{2}}{E \cdot t \cdot \cos (\alpha r)} \cdot\left[(h+H-z)-\frac{v}{2} \cdot\left(h+H-\frac{2}{3} \cdot z\right)\right]
\end{aligned}
$$

- equação das rotações totais ao longo do meridiano da superficie média da casca cônica (força, momento e líquido armazenado) em [radianos] : 


$$
\begin{gathered}
\phi a(z)=\frac{\text { Hca. } \cos (\alpha r)}{2 \cdot \alpha^{2} \cdot B} \cdot e^{-\alpha \cdot \frac{H-z}{\cos (\alpha r)}} \cdot\left[\cos \left(\alpha \cdot \frac{H-z}{\cos (\alpha r)}\right)+\sin \left(\alpha \cdot \frac{H-z}{\cos (\alpha r)}\right)\right]+ \\
-\frac{\gamma \cdot \tan (\alpha r)^{2} \cdot z}{6 \cdot E \cdot t \cdot \cos (\alpha r)} \cdot(-9 \cdot h-9 \cdot H+16 \cdot z)
\end{gathered}
$$

- equação dos momentos solicitantes ao longo dos paralelos da superficie média da casca cônica para esforços distribuídos na borda superior apoiada fixa em [ton. $\mathrm{cm} / \mathrm{cm}$ ]

$$
\operatorname{Mla}(z)=\frac{\text { Hca } \cdot \cos (\alpha r)}{\alpha} \cdot e^{-\alpha \cdot \frac{H-z}{\cos (\alpha r)}} \cdot \sin \left(\alpha \cdot \frac{H-z}{\cos (\alpha r)}\right)
$$

- equação dos momentos solicitantes ao longo dos meridianos da superfície média da casca cônica para esforços distribuídos na borda superior apoiada fixa em [ton. $\mathrm{cm} / \mathrm{cm}]$ :

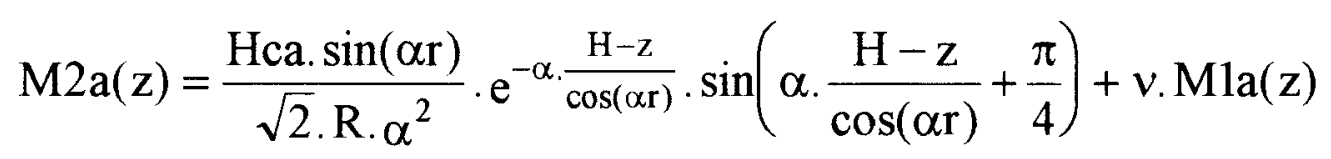

- equação das forças solicitantes ao longo dos meridianos da superficie média da casca cônica para esforços distribuídos na borda apoiada fixa em $[$ ton $/ \mathrm{cm}]$ :

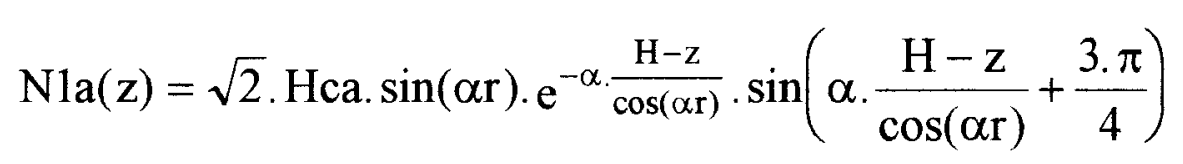

- equação das forças solicitantes ao longo dos paralelos da superficie média da casca cônica para esforços distribuídos na borda apoiada fixa em $[\mathrm{ton} / \mathrm{cm}]$ : 


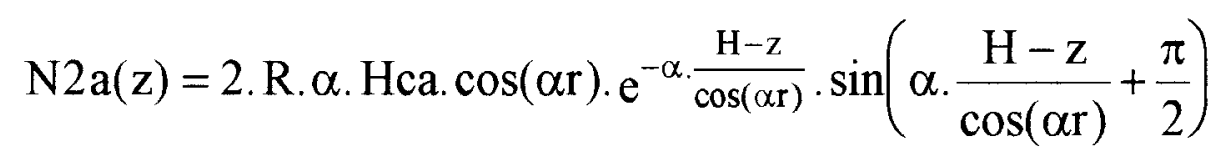

- equação das tensões ao longo dos meridianos das superfícies interna (índice "in") e externa (índice "ex") da casca cônica em [ton/ $\left.\mathrm{cm}^{2}\right]$

$$
\begin{aligned}
& \sigma \operatorname{ain}(z):=\frac{\gamma \cdot \tan (\alpha r) \cdot z}{2 \cdot t \cdot \cos (\alpha r)} \cdot\left(h+H-\frac{2}{3} \cdot z\right)+\frac{N 1 a(z)}{t}-\frac{6 \cdot M 1 a(z)}{t^{2}} \\
& \sigma 1 \operatorname{aex}(z):=\frac{\gamma \cdot \tan (\alpha r) \cdot z}{2 \cdot t \cdot \cos (\alpha r)} \cdot\left(h+H-\frac{2}{3} \cdot z\right)+\frac{N 1 a(z)}{t}+\frac{6 \cdot M 1 a(z)}{t^{2}}
\end{aligned}
$$

- equação das tensões ao longo dos paralelos das superficies interna (índice "in") e externa (índice "ex") da casca cônica em [ton $\left./ \mathrm{cm}^{2}\right]$ :

$$
\begin{aligned}
& \sigma 2 \operatorname{ain}(z):=\frac{\gamma \cdot \tan (\alpha r) \cdot z}{t \cdot \cos (\alpha r)} \cdot(h+H-z)+\frac{N 2 a(z)}{t}-\frac{6 \cdot M 2 a(z)}{t^{2}} \\
& \sigma 2 a \operatorname{aex}(z):=\frac{\gamma \cdot \tan (\alpha r) \cdot z}{t \cdot \cos (\alpha r)} \cdot(h+H-z)+\frac{N 2 a(z)}{t}+\frac{6 \cdot M 2 a(z)}{t^{2}}
\end{aligned}
$$

\subsubsection{TENSÕES TEÓRICAS DE MEMBRANA}

Como para as cascas cilíndricas, as expressões das tensões teóricas de membrana para a casca cônica sob ação da carga hidrostática do produto armazenado, são facilmente encontradas na resistência dos materiais, e apresentadas abaixo (índice "m") :

- equação das tensões de membrana ao longo dos meridianos da superficie média da casca cônica em [ton $\left./ \mathrm{cm}^{2}\right]$ : 


$$
\sigma \operatorname{lm}(\mathrm{z}):=\frac{\gamma \cdot \tan (\alpha \mathrm{r}) \cdot \mathrm{z}}{2 \cdot \mathrm{t} \cdot \cos (\alpha \mathrm{r})} \cdot\left(\mathrm{h}+\mathrm{H}-\frac{2}{3} \cdot \mathrm{z}\right)
$$

- equação das tensões de membrana ao longo dos paralelos da superficie média da casca cônica em [ton $\left./ \mathrm{cm}^{2}\right]$ :

$$
\sigma 2 m(z):=\frac{\gamma \cdot \tan (\alpha r) \cdot z}{t \cdot \cos (\alpha r)} \cdot(h+H-z)
$$

Utilizando-se das equações de (34) a (66), pode-se resolver estruturalmente a maioria das cascas cônicas delgadas, com borda superior engastada ou apoiada fixa, submetidas a ação de pressão hidrostática, desde que, lembrando novamente, possuam características que atendam os comentários do item 2.1 . 


\section{ANÁLISE POR ELEMENTOS FINITOS}

\subsection{CONSIDERAÇÕES GERAIS}

No capítulo anterior obteve-se, através da teoria de estruturas em casca de revolução, o equacionamento das expressões teóricas específicas para o cálculo estrutural de cascas cilíndrica e cônica, com vinculação de borda engastada e articulada fixa

Neste capítulo utilizou-se, na análise dessas mesmas estruturas, o Método dos Elementos Finitos através do programa "comercial", bastante conhecido pelos engenheiros de cálculo estrutural, SAP90 (Structural Analysis Program) desenvolvido por Edward L. WILSON e Ashraf HABIBULLAH da Universidade da California, Berkeley

Esse programa, por ter sido utilizado por centenas de firmas de engenharia em todo o mundo, adquiriu grande reputação na família dos programas de computador para análise estrutural.

Optou-se pelo SAP90, uma das ferramentas de análise estrutural por elementos finitos disponível no momento, por ser suficiente para este estudo introdutório, feito de forma a obedecer as hipóteses fundamentais de "Kirchoff-Love" apresentadas nas considerações gerais do capítulo anterior (item 2.1), considerando teoria linear, efeitos de primeira ordem e carregamento estático.

Hoje, já encontra-se o SAP na sua mais nova versão, o SAP2000, atualizado para um ambiente mais amigável, com novos recursos e também para análises nãolineares das estruturas.

O Método dos Elementos Finitos (MEF), utilizado na análise de estruturas, atinge seus objetivos, considerando a estrutura dividida em partes finitas (elementos) 
ligadas entre sí por pontos nodais (nós) onde se supõe aplicadas as ações ou reações A composição desses elementos para constituir a estrutura dá lugar a um sistema de equações que são tratados matricialmente.

Para identificar e ordenar matricialmente as ações mecânicas e os deslocamentos existentes nos nós de uma estrutura integrada ou nas extremidades de um elemento, quando a estrutura é subdividida, utiliza-se um sistema de referência chamado "sistema de coordenadas".

O sistema de referência adotado que define direções e sentidos associados aos nós da estrutura integrada representa as "coordenadas globais". O sistema que define direções e sentidos associados às extremidades dos elementos da estrutura desmembrada representa as "coordenadas locais"

Os "vínculos" são os nós da estrutura escolhidos para satisfazer as condições de contorno que sustentam a mesma no espaço, restringindo o deslocamento do nó escolhido nas coordenadas globais desejadas para satisfazer essas condições. Normalmente os vínculos são chamados de apoios, que podem ser engastes, articulações, apoios móveis ou fixos, conforme a combinação de coordenadas bloqueadas.

O programa SAP90 necessita da definição dos itens citados anteriormente, e isso é feito através da digitação de uma entrada de dados com comandos específicos encontrados no manual do usuário que acompanha o programa.

Ao utilizar-se o MEF para a análise de uma estrutura, é importante a definição do tamanho dos elementos que compõem a casca. Por utilizar elementos "finitos" o MEF também é um método aproximado e quanto maior a experiência do projetista na geração da malha de elementos, maior é a sensibilidade do modelo na representação do comportamento da estrutura.

Para poder comparar as expressões teóricas do capítulo 2 e o MEF, chegouse, após várias tentativas, aos seguintes valores para altura dos elementos da malha :

- para a casca cilíndrica : malha de elementos com 5 [cm] de altura cada um.

- para a casca cônica : malha de elementos com $\frac{5}{\cos \theta r}[\mathrm{~cm}]$ de altura cada um, onde $\theta$ r é o ângulo da casca cônica com a horizontal. 
As malhas assim definidas foram suficientes para permitir a comparação dos resultados de deslocamentos, esforços solicitantes e tensões atuantes obtidos pelas duas ferramentas de cálculo

Para um melhor entendimento dos conceitos utilizados no MEF, e dos comandos do programa, descrever-se-á passo a passo a codificação dos dados de entrada feita para solução das estruturas deste trabalho, como será visto a seguir.

\subsection{CODIFICAÇ̃̃O PARA CASCA CILÍNDRICA}

Para a solução de estruturas por elementos finitos, existe a necessidade da definição previa das dimensões das mesmas, de forma que se possua todos os parâmetros exigidos na codificação da entrada de dados do programa.

Utilizou-se as dimensões de uma casca cilíndrica "típica" da área de processos da indústria sucro-alcooleira

Material da casca cilíndrica : Aço carbono ASTM A283C

Produto armazenado : adotado água

$\mathrm{E}=2100-$ modulo de elasticidade $\mathrm{em}\left[\mathrm{ton} / \mathrm{cm}^{2}\right.$ ]

$v=0,3$ - coeficiente de Poisson

$\gamma=1.10^{-6}$ - peso específico do produto armazenado em $\left[\mathrm{ton} / \mathrm{cm}^{3}\right]$

$\mathrm{D}=1200$ - diâmetro da superficie média da casca em [cm]

$\mathrm{R}=600$ - raio da superficie média da casca em $[\mathrm{cm}]$

$\mathrm{H}=1200$ - altura total da casca $\mathrm{em}[\mathrm{cm}]$

$\mathrm{h}=1200-$ altura da coluna de produto armazenado em $[\mathrm{cm}]$

$\mathrm{t}=1$ - espessura (constante) da casca em $[\mathrm{cm}]$

Z - eixo vertical medido a partir da borda vinculada coincidente com o eixo de rotação da casca

$\mathrm{X}$ - eixo horizontal pertencente ao plano do papel, medido a partir do início do eixo vertical " $Z$ "

Y - eixo horizontal que fura o plano do papel, medido a partir do início do eixo vertical "Z" 


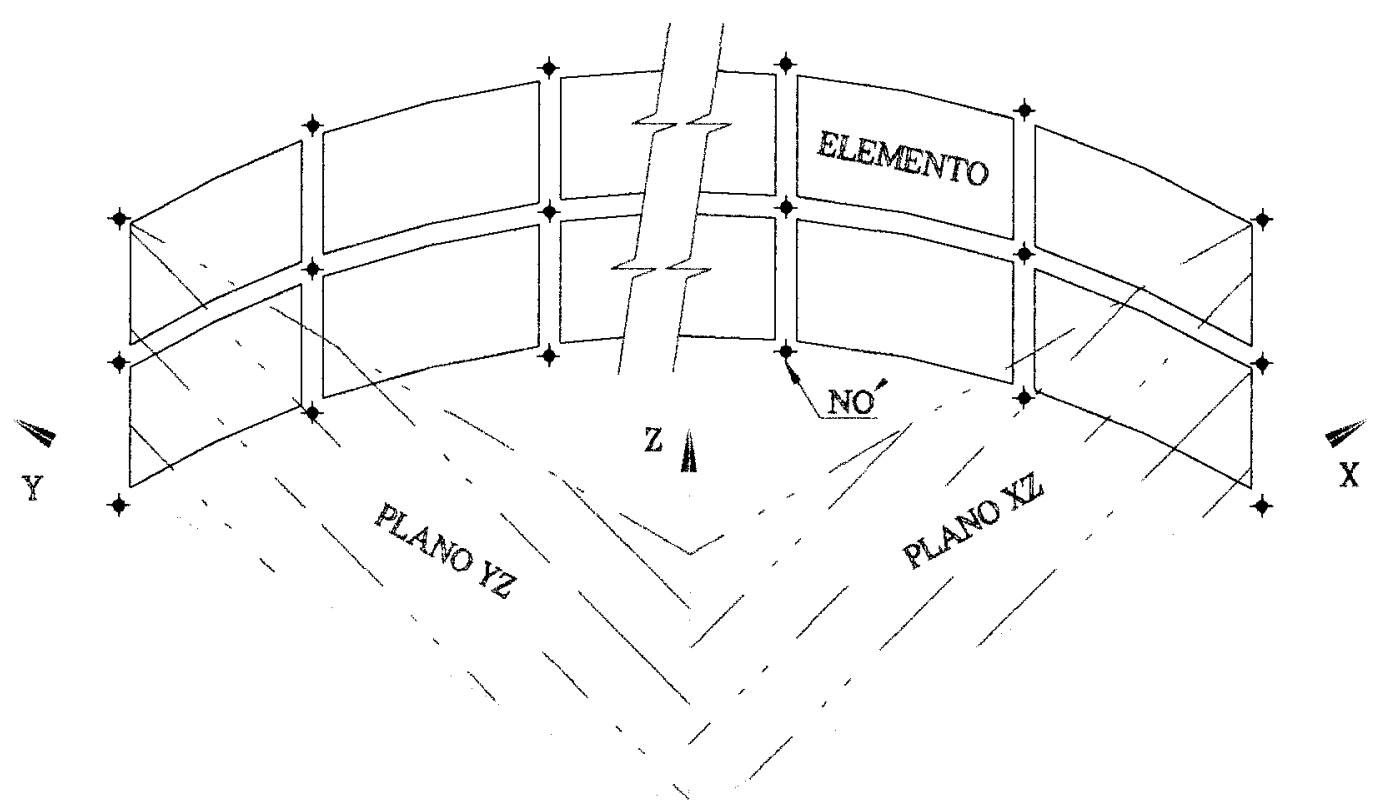

FIGURA 22 - Esquema geral para modelo da casca cilíndrica

O programa permitiu fazer a análise estrutural das cascas cilíndrica e cônica através da geração de apenas um de seus quadrantes por serem de revolução e possuírem carregamento simétrico em relação ao seu eixo (pressão hidrostática),

Os três quadrantes restantes foram simulados através de vinculações que não permitissem que os nós pertencentes aos planos de corte do quadrante estudado saíssem desses planos como pode-se ver adiante.

É importante observar, que a casca cilíndrica não foi gerada na sua altura total de $\mathrm{H}=1200[\mathrm{~cm}]$, mesmo tendo sido carregada com uma coluna de água de $\mathrm{h}=1200$ [cm]. Isto se deveu ao fato das expressões téoricas aproximadas, desenvolvidas no capítulo 2, terem fornecido resultados praticamente idênticos aos obtidos pelo MEF para alturas da casca maiores que " $\lambda$ " (da expressão 4 encontra-se 119,73 [cm]). A análise da casca cilíndrica foi limitada, portanto, até uma altura de aproximadamente "2. $\lambda$ " (adotou-se $240[\mathrm{~cm}]$ )

De posse dessas informações poder-se-á então apresentar a codificação de entrada no programa. 


\subsubsection{ARQUIVO PARA ENTRADA DE DADOS}

A entrada de dados será dividida em blocos para facilidade de compreensão :

- $\mathbf{1}^{0}$ BLOCO : Título da estrutura em estudo

Casca cilíndrica $\mathrm{D}=1200 \times \mathrm{h}=1200$ - Produto : Água

C Vinculação da borda inferior : Engastada ou apoiada fixa

- $\mathbf{2}^{\circ}$ BLOCO : Definição do número de carregamentos

SYSTEM

$\mathrm{L}=1$

Considerou-se apenas a ação da pressão hidrostática da água, portanto somente um carregamento, " $\mathrm{L}=1$ ". O pêso próprio da casca cilíndrica foi desprezado para permitir a comparação dos resultados obtidos pelo MEF com os das expressões teóricas do capítulo 2 .

- $3^{0}$ BLOCO : Geração dos pontos nodais da estrutura JOINTS

C Eixo de rotação

\begin{tabular}{|c|c|c|c|}
\hline 1 & $X=0$ & $\mathrm{Y}=0$ & $Z=-10$ \\
\hline 1000 & & & $Z=250$ \\
\hline
\end{tabular}

Esses nós devem ser gerados para simular o eixo de rotação da casca cilíndrica. São os nós que permitem utilizar o recurso de geração cilíndrica do programa.

C Nós da casca cilíndrica

\begin{tabular}{|c|c|c|c|c|}
\hline 2 & $X=600$ & $Y=0$ & $Z=0$ & $A=1,1000,2,10,1,9$ \\
\hline & & & $Z=5$ & $A=1,1000,13,10,1,9$ \\
\hline & & & $Z=10$ & $\mathrm{~A}=1,1000,24,10,1,9$ \\
\hline
\end{tabular}




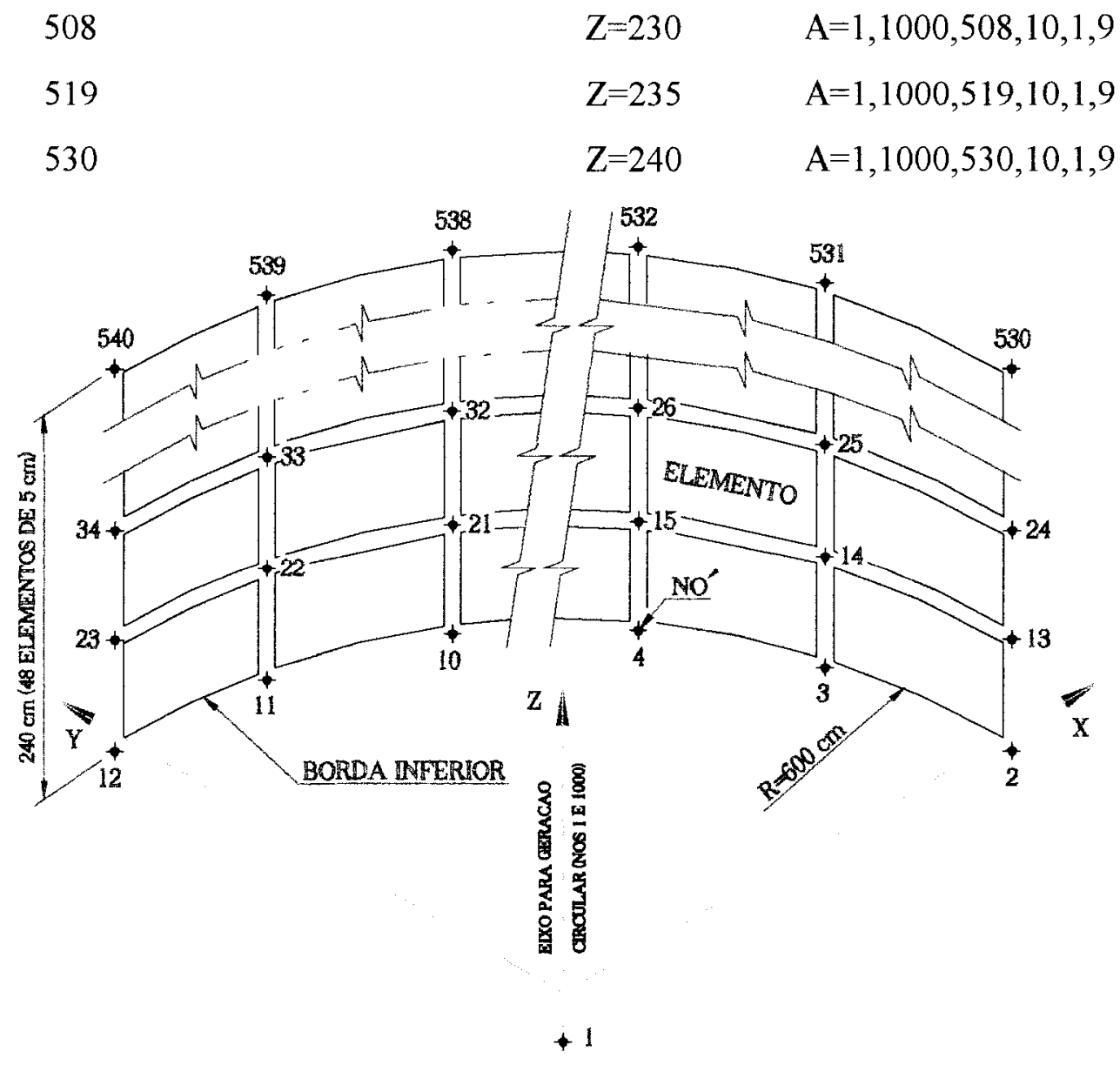

FIGURA 23 - Esquema da geração dos nós

Os nós de 2 a 530 são os nós da casca cilíndrica propriamente dita. As coordenadas dos nós no sistema global são definidas pelos valores de $\mathrm{X}, \mathrm{Y}$ e $\mathrm{Z}$, onde o valor de $X$ representa o raio da superfície média da casca $(600 \mathrm{~cm})$.

$\mathrm{A}=\mathrm{c}_{1}, \mathrm{c}_{2}, \mathrm{c}_{3}, \mathrm{n}_{\mathrm{c}}, \mathrm{i}_{\mathrm{c}}, \mathrm{a}$ é o comando para geração circular e significa :

$c_{1}$ - nó inicial que define o eixo de rotação simulado (1)

$c_{2}$ - nó final que define o eixo de rotação simulado (1000)

$c_{3}$ - nó inicial da geração (2 a 530)

$\mathrm{n}_{\mathrm{c}}$ - número de nós adicionais a serem gerados $(10)$

$\mathrm{i}_{\mathrm{c}}$ - incremento para a numeração dos nós (1)

a - ângulo de incremento da geração em graus (9)

Com isso, por exemplo, para $\mathrm{A}=1,1000,2,10,1,9$ foram gerandos os primeiros 11 nós da casca cilíndrica na coordenada global $Z=0$. 
- $4^{0}$ BLOCO : Vinculação dos nós da estrutura

\section{RESTRAINTS}

C Nós do eixo de rotação

$11000999 \mathrm{R}=1,1,1,1,1,1$

Os nós de 1 a 1000, com incremento de 999 , representam a simulação do eixo de rotação da casca, e como não farão parte da estrutura, estão com todos os seus graus de liberdade restringidos $(\mathrm{R}=1,1,1,1,1,1)$.

A vinculação é simulada pelo comando $R=r_{u x}, r_{u y}, r_{u z}, r_{r x}, r_{r y}, r_{r z}$, onde :

$r_{u x}, r_{u y}$ e $r_{u z}$ - são respectivamente translações nos eixos $X, Y$ e $Z$ globais

$r_{r x}, r_{r y}$ e $r_{r z}$ - são respectivamente as rotações em torno dos eixos $X, Y$ e Z globais

O valor " 1 " significa a restrição do movimento no eixo desejado e o valor "0" significa a liberação do seu movimento.

C Nós da casca cilíncrica

$$
2530 \quad 11 \quad \mathrm{R}=0,1,0,1,0,1
$$

Os nós de 2 a 530, com incremento de 11, podem se deslocar em relação ao eixo $X$, não podem em relação a $Y$ e podem em relação a $Z$. Não podem girar em torno do eixo $X$, podem em relação a $\mathrm{Y}$ e não podem em relação a $Z$. Esta vinculação os impede de sairem do plano $\mathrm{XZ}$, simulando os quadrantes faltantes da casca, que como já foi dito não precisam ser gerados para estruturas de revolução com carregamento simétrico em relação ao seu eixo.

$12540 \quad 11 \mathrm{R}=1,0,0,0,1,1$

Os nós de 12 a 540, com incremento de 11 , não podem se deslocar em relação ao eixo $X$, podem em relação a $\mathrm{Y}$ e a $\mathrm{Z}$. Podem girar em torno do eixo $\mathrm{X}$, não podem em relação a Y e a Z. Esta vinculação os impede de sairem do plano YZ, também simulando os quadrantes faltantes da casca como anteriormente para os nós de 2 a 530.

$$
2121 \mathrm{R}=1,1,1,1,1,1
$$

As condições de contorno consideradas neste trabalho, já definidas no capítulo 2, são aqui representadas : os nós de 2 a 12 , com incremento de 1 , compõem a borda 
inferior da casca cilíndrica, que será vinculada por um engaste $(R=1,1,1,1,1,1)$ e posteriormente por um apoio articulado fixo $(R=1,1,1,0,0,0)$.

- $5^{\circ}$ BLOCO : Geração dos elementos da estrutura

\section{SHELL}

$\mathrm{NM}=1 \quad \mathrm{O}=0 \quad \mathrm{P}=-1$

"NM" define o número de tipos de materiais que compõem a estrutura, no caso, apenas um : aço carbono.
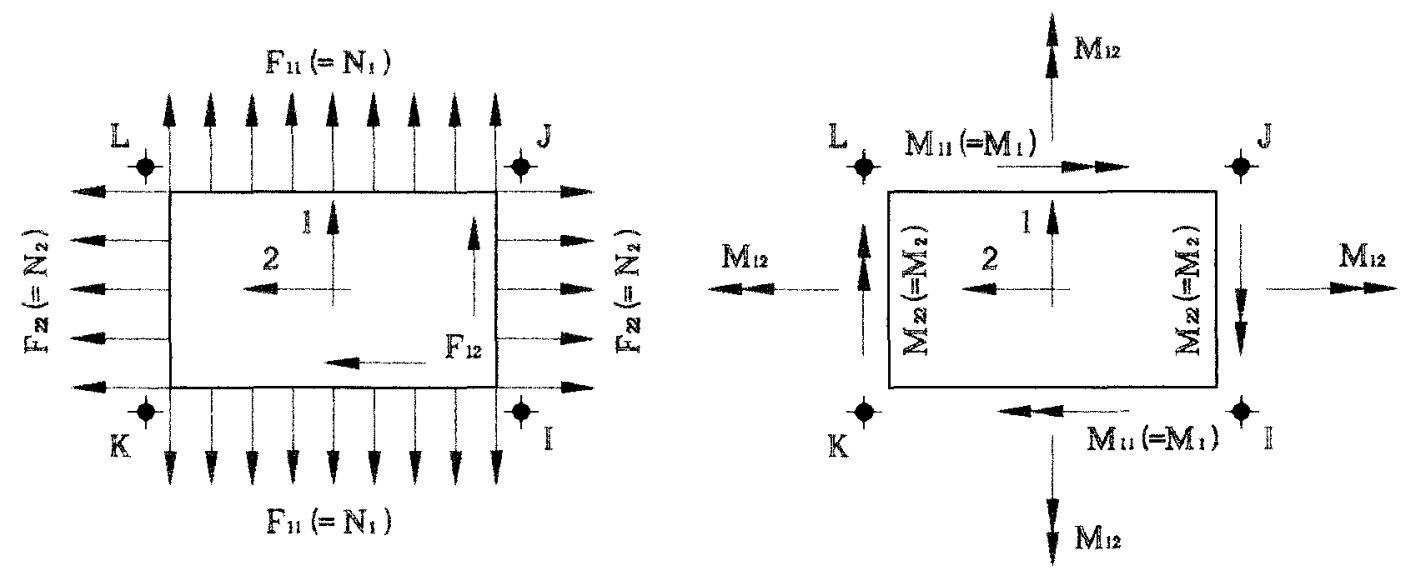

FIGURA 24 - Esquema dos esforços fornecidos na saída de dados para opção "O=0" ("O" igual a zero)

A letra "O" define como será a saída de dados após o processamento do programa. Para "O=0" ("O" igual a zero) o programa fornecerá as forças e momentos solicitantes em cada nó do elemento. Para " $\mathrm{O}=1$ " fornecerá a saída de dados com as tensões atuantes nos nós das duas faces do elemento, superior e inferior, ou para a casca cilíndrica, internamente e externamente a mesma.

"P" define os multiplicadores para carregamentos de pressão. Com "P=-1" fica definido que a pressão hidrostática do produto armazenado se dirige de dentro para fora da casca cilíndrica (pressão interna).

$$
1 \quad \mathrm{E}=2100 \quad \mathrm{U}=0.3
$$

Esta linha define as propriedades do material "1" (aço), como o módulo de elasticidade "E" e o coeficiente de Poisson, simbolizado por "U". 


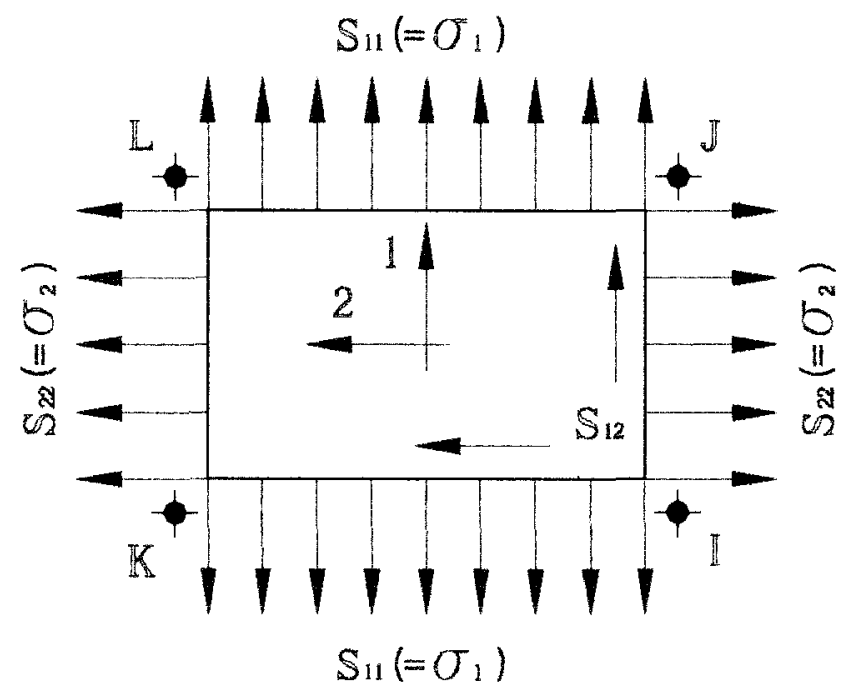

FIGURA 25 - Esquema dos esforços fornecidos na saída de dados para opção " $\mathrm{O}=1$ " para as faces interna (top) e externa (bot) do elemento

$$
1 \mathrm{JQ}=2,13,3,14 \quad \text { ETYPE }=0 \quad \mathrm{M}=1 \quad \mathrm{TH}=1,1 \quad \mathrm{LP}=0 \quad \mathrm{G}=48,10
$$

A linha acima significa que o elemento número "1" é criado pelo comando $J Q=j_{l}, j_{J}, j_{K}, j_{L}$, onde $j_{l}, j_{j}, j_{K}, j_{L}$ são os números dos nós que compõem o elemento, nas suas respectivas extremidades $\mathrm{I}, \mathrm{J}, \mathrm{K}$ e L

O comando "ETYPE" define, se igual a "0", comportamento de membrana e solicitações de flexão, se igual a "1", apenas comportamento de membrana, e, se igual a "2", apenas solicitações de flexão na casca. Considerou-se "ETYPE=0".

"M" define o tipo do material do elemento da casca, no caso material "1".

A espessura, $t=1 \mathrm{~cm}$ da casca, deve ser especificada, para comportamento de membrana e para solicitações de flexão, através do comando " $\mathrm{TH}=\mathrm{t}_{\mathrm{h} 1}, \mathrm{t}_{\mathrm{h} 2}$ ", sendo $\mathrm{t}_{\mathrm{h} 1}$ espessura para membrana e $t_{\mathrm{h} 2}$ para flexão

Os eixos locais dos elementos, eixos "1, 2 e 3", são considerados como partindo do centro do elemento, e seguem a regra da mão direita para sua definição. $\mathrm{O}$ vetor V3 que representa o eixo local "3", tem sempre sua direção normal ao plano do elemento da casca. "LP=0" indica que o vetor V1, que representa o eixo "1", vai do ponto central do lado I-K do elemento para o ponto central do seu lado J-L. Com o produto vetorial de V3 por V1 define-se então a direção e sentido do vetor V2, correspondente ao eixo "2". E assim, direções e sentidos dos eixos locais do elemento, são obtidas. 


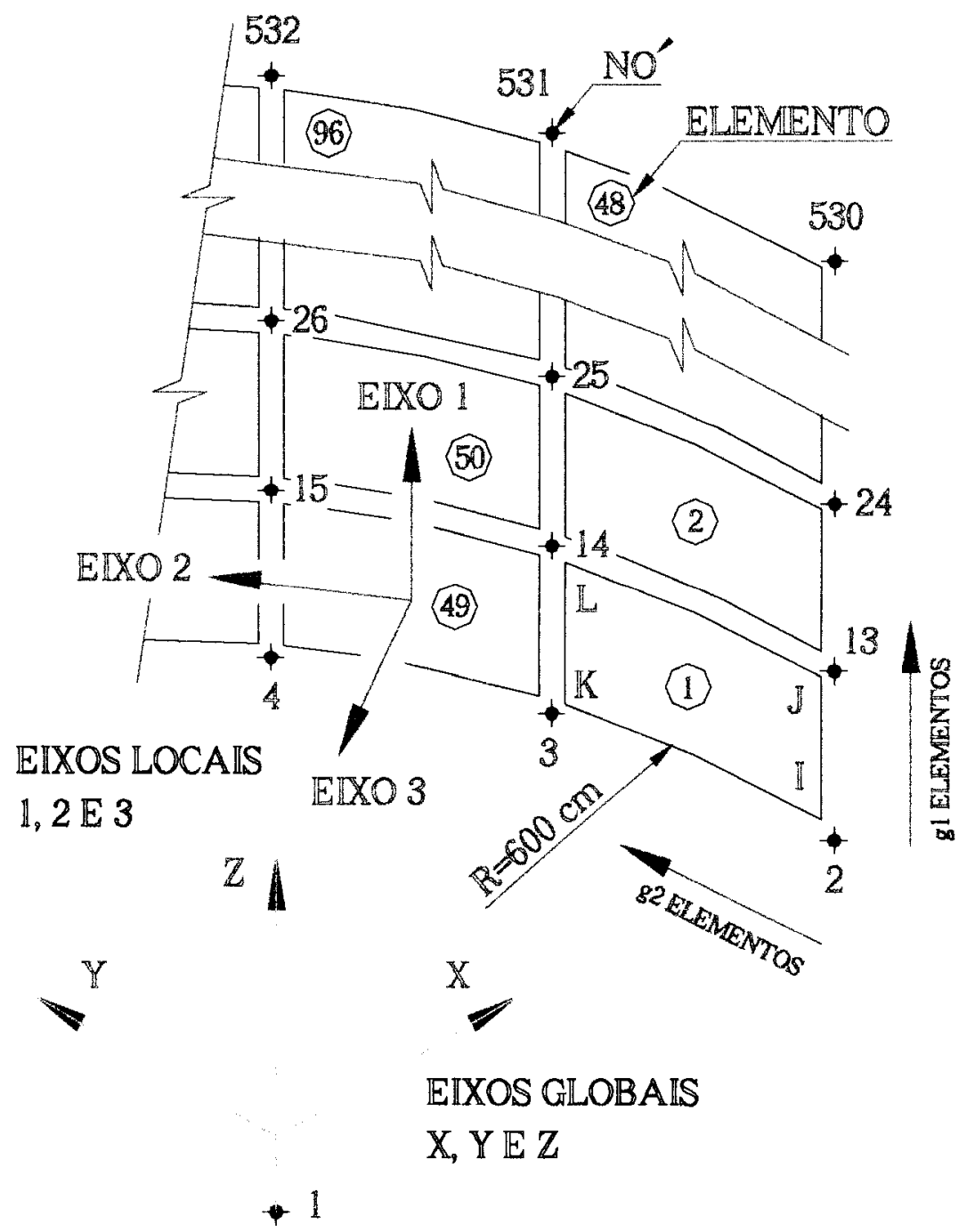

FIGURA 26 - Esquema da geração dos elementos

Para geração de elementos do tipo "SHELL" utiliza-se o comando " $\mathrm{G}=\mathrm{g}_{1}, \mathrm{~g}_{2}$ ", sendo $g_{1}$ e $g_{2}$ parâmetros que criam uma malha bi-dimensional com $g_{1}$ elementos na direção I-J do elemento gerador, e $\mathrm{g}_{2}$ elementos na direção $\mathrm{I}-\mathrm{K}$. Com " $\mathrm{G}=48,10$ ", foram criados 48 elementos na direção I-J (na direção do meridiano do quadrante cilíndrico ou sentido positivo do eixo global Z) e 10 elementos na I-K (na direção do paralelo do quadrante cilíndrico).

- $6^{0}$ BLOCO : Ação dos carregamentos na estrutura POTENTIAL

$2540 \quad 1 \quad \mathrm{~W}=1 \mathrm{E}-06,1200$ 
A casca cilíndrica está sendo carregada, do nó 2 ao 540 , com incremento de 1 , com pressão hidrostática causada por uma coluna de água de peso específico $\gamma=$ $1.10^{-6}\left[\mathrm{ton} / \mathrm{cm}^{3}\right]$ e altura de $1200[\mathrm{~cm}]$, ou seja "W=1E-06,1200".

Fica assim definida a entrada de dados com os comandos necessários para que o programa SAP90 possa resolver a casca cilindrica de revolução, sob a ação de pressão hidrostática, vinculada continuamente na sua borda inferior primeiramente por um engastamento $(\mathrm{R}=1,1,1,1,1,1)$ e depois por apoio articulado fixo $(\mathrm{R}=1,1,1,0,0,0)$.

\subsection{CODIFICAÇÃO PARA CASCA CÔNICA}

A partir das dimensões da casca cilíndrica foi utilizado para a casca cônica :

Material da casca cilíndrica : Aço carbono ASTM A283C

Produto armazenado : adotado água

$\mathrm{E}=2100-$ modulo de elasticidade em $\left[\mathrm{ton} / \mathrm{cm}^{2}\right]$

$v=0,3$ - coeficiente de Poisson

$\gamma=1.10^{-6}$ - peso específico do produto armazenado em [ton $\left./ \mathrm{cm}^{3}\right]$

$\alpha r=30,45$ e 60 - ângulo de metade do bico da casca cônica com a vertical em [graus] (codificou-se para três diferentes inclinações da casca)

$\theta r=(90-\alpha r)=60,45$ e 30 - ângulo de metade do bico da casca cônica com a horizontal em [graus] (codificou-se para três diferentes inclinações da casca)

$\mathrm{D}=1200$ - diâmetro da superficie média da borda superior da casca cônica $\mathrm{em}[\mathrm{cm}]$

$r=\frac{D}{2}=600-$ raio da superficie média da borda superior da casca cônica $\mathrm{em}[\mathrm{cm}]$

$\mathrm{R}=\frac{\mathrm{D}}{2 \cdot \cos (\alpha r)}-$ raio projetado da superfície média da borda superior da casca cônica em [cm] (variável com $\alpha \mathrm{r}$ ) 


$$
\begin{gathered}
H=\frac{D}{2 \cdot \tan (\alpha r)}-\text { altura total da casca cônica em }[\mathrm{cm}](\text { variável com } \alpha \mathrm{r}) \\
\mathrm{h}=1200-\text { altura da coluna de produto armazenado acima da casca } \\
\text { cônica em }[\mathrm{cm}]
\end{gathered}
$$

$\mathrm{h}+\mathrm{H}$ - altura total da coluna de produto armazenado em [cm] (variável com $\alpha r)$

$\mathrm{t}=1$ - espessura (costante) da casca cônica em $[\mathrm{cm}]$

$Z$ - eixo vertical medido a partir do bico da casca cônica coincidente com o seu eixo de rotação

$X$ - eixo horizontal pertencente ao plano do papel, medido a partir do início do eixo vertical " $Z$ "

Y - eixo horizontal que fura o plano do papel, medido a partir do início do eixo vertical " $Z$ "

A casca cônica foi gerada na sua altura total $\mathrm{H}$ e carregada com uma coluna de água de $h+H$.

\subsubsection{ARQUIVO PARA ENTRADA DE DADOS}

Os blocos da entrada de dados são semelhantes aos apresentados para a casca cilíndrica, portanto, serão comentados apenas os que possuírem características de aplicação específicas para a casca cônica.

- 1 $^{\mathrm{o}}$ BLOCO : Título da estrutura em estudo (exemplo para $\theta \mathrm{r}=30$ [graus])

Casca cônica 30 graus com horizontal $\mathrm{D}=1200$ - Produto : Água

C Vinculação da borda superior : Engastada ou apoiada fixa 
- $2^{\circ}$ BLOCO : Definição do número de carregamentos

SYSTEM

$\mathrm{L}=1$

- $\mathbf{3}^{0}$ BLOCO : Geração dos pontos nodais da estrutura

\section{JOINTS}

C Eixo de rotação

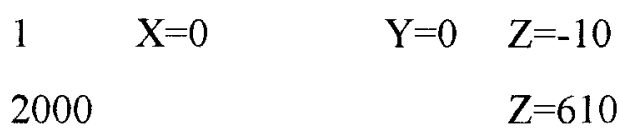

C Nós da casca cônica

$$
\begin{aligned}
& 2 \quad \mathrm{X}=0 \quad \mathrm{Y}=0 \quad \mathrm{Z}=0 \\
& 3 \quad \mathrm{X}=5 \quad \mathrm{Z}=5 * 0.57735027 \quad \mathrm{~A}=1,2000,3,10,1,9 \\
& 14 \quad X=10 \quad Z=10 * 0.57735027 \quad A=1,2000,14,10,1,9
\end{aligned}
$$

$1290 \quad X=590 \quad Z=590 * 0.57735027 \quad A=1,2000,1290,10,1,9$

$1301 \mathrm{X}=595 \quad \mathrm{Z}=595 * 0.57735027 \quad \mathrm{~A}=1,2000,1301,10,1,9$

$1312 X=600 \quad Z=600 * 0.57735027 \quad A=1,2000,1312,10,1,9$

O valor 0,57735027 multiplicado pelas coordenadas $X$ (de 5 a 600), corresponde à tangente do ângulo $\theta \mathrm{r}$, nesse exemplo tangente de 30 [graus].

$-4^{0}$ BLOCO : Vinculação dos nós da estrutura

\section{RESTRAINTS}

C Nós do eixo de rotação

$12000 \quad 1999 \quad \mathrm{R}=1,1,1,1,1,1$

C Nós da casca cônica

2

$$
\mathrm{R}=1,1,0,1,1,1
$$

Este nó define o bico da casca cônica, pertencendo aos dois planos $\mathrm{XZ}$ e $\mathrm{YZ}$, não podendo, portanto, deslocar em relação aos eixos $\mathrm{X}$ e $\mathrm{Y}$ mas podendo deslocar em relação a $Z$. Não pode girar em torno dos três eixos. 


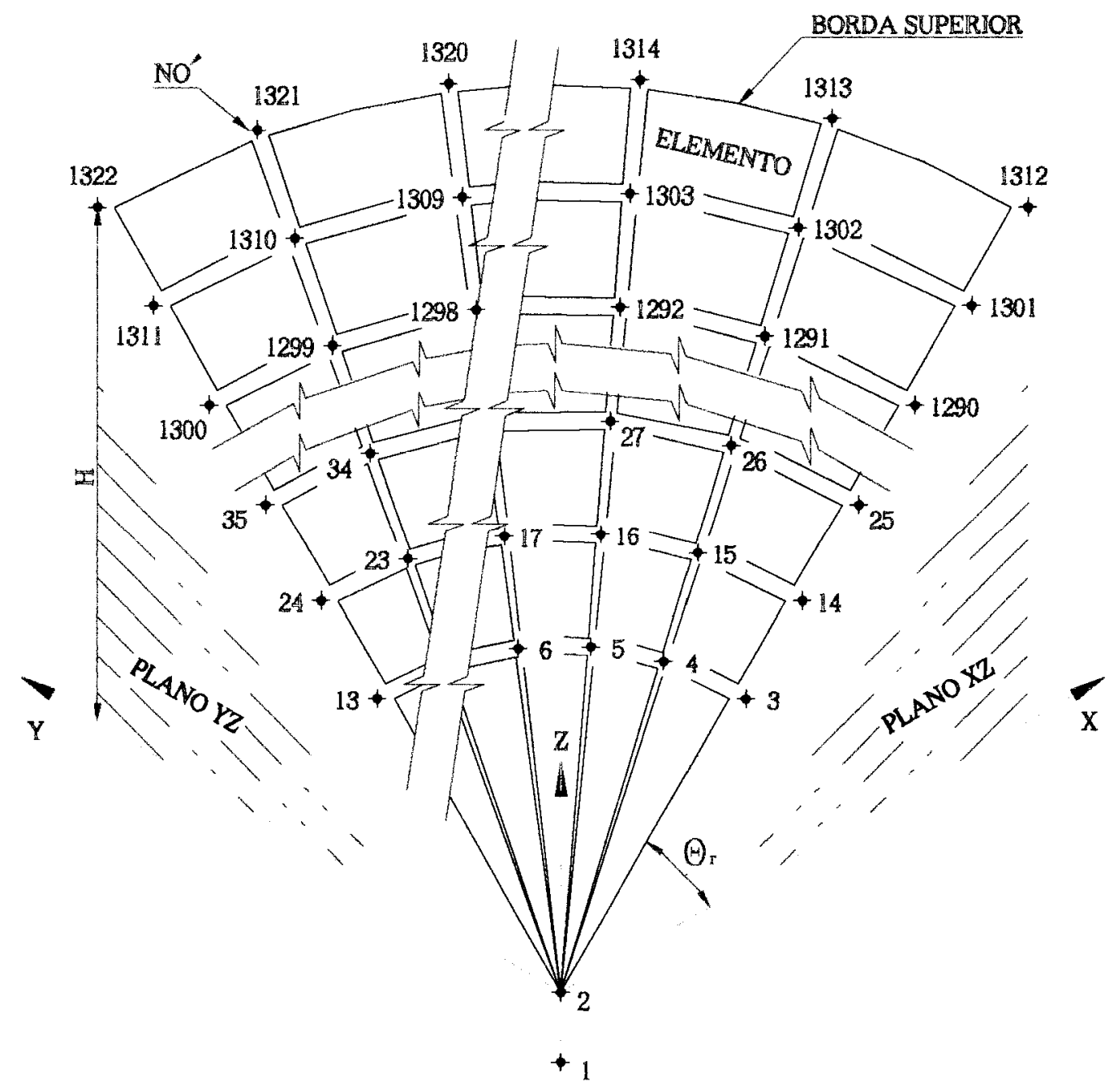

FIGURA 27 - Esquema da geração dos nós

$3 \quad 131211 \quad \mathrm{R}=0,1,0,1,0,1$

Esta vinculação impede os nós de 3 a 1312 de sairem do plano $\mathrm{XZ}$, simulando os quadrantes faltantes da casca.

$13 \quad 1322 \quad 11 \quad \mathrm{R}=1,0,0,0,1,1$

Esta impede os nós de 13 a 1322 de sairem do plano YZ, também simulando os quadrantes faltantes da casca.

$$
13121322 \quad 1 \quad \mathrm{R}=1,1,1,1,1,1
$$

Os nós de 1312 a 1322, com incremento de 1, compõem a borda superior da casca cônica, que será vinculada por um engaste $(R=1,1,1,1,1,1)$ e posteriormente por um apoio articulado fixo $(R=1,1,1,0,0,0)$. 
- $5^{0}$ BLOCO : Geração dos elementos da estrutura

SHELL

$$
\begin{aligned}
& \text { NM=1 } \quad \mathrm{O}=0 \quad \mathrm{P}=1 \\
& 1 \quad \mathrm{E}=2100 \quad \mathrm{U}=0.3 \\
& \text { C Casca cônica }
\end{aligned}
$$

$$
1 \mathrm{JQ}=1312,1301,1313,1302 \quad \text { ETYPE }=0 \quad \mathrm{M}=1 \quad \mathrm{TH}=1,1 \quad \mathrm{LP}=0 \quad \mathrm{G}=119,10
$$

Como na casca cilíndrica, os eixos locais dos elementos "1, 2 e 3", partem do centro do elemento, e seguem a regra da mão direita para sua definição. O vetor V3 tem sempre sua direção normal ao plano do elemento da casca. "LP=0" indica que V1 vai do ponto central do lado I-K do elemento para o ponto central do seu lado J-L. Com o produto vetorial de V3 por V1 define-se a direção e o sentido de V2.

Observar que, enquanto para "LP=0", V3 se dirigia de fora para dentro na casca cilíndrica, na casca cônica se dirige de dentro para fora. Portanto, em função do modo como a casca cônica foi gerada, fica definido que a pressão hidrostática do

\begin{tabular}{|c|c|c|c|c|}
\hline 1191 & $\mathrm{JQ}=3,2,4$ & ETYPE $=0$ & $M=1$ & $\mathrm{TH}=1,1$ \\
\hline 1192 & $\mathrm{JQ}=4,2,5$ & $\mathrm{ETYPE}=0$ & $M=1$ & $\mathrm{TH}=1,1$ \\
\hline - & & & $\cdot$ & - \\
\hline • & & & - & - \\
\hline - & & & - & - \\
\hline 1199 & $\mathrm{JQ}=11,2,12$ & $\mathrm{ETYPE}=0$ & $M=1$ & $\mathrm{TH}=1,1$ \\
\hline 1200 & $\mathrm{JQ}=12,2,13$ & $\mathrm{ETYPE}=0$ & $M=1$ & $\mathrm{TH}=1,1$ \\
\hline
\end{tabular}
produto armazenado se dirige de dentro para fora da casca (pressão interna) somente com "P=1", ou seja no sentido positivo do eixo V3.

C Fechamento do bico da casca cônica

Para o fechamento do bico da casca cônica utilizou-se elementos do tipo "SHELL" triangulares, criados pelo comando $J Q=\mathrm{j}_{\mathrm{r}}, \mathrm{j}_{\mathrm{J}}, \mathrm{j}_{\mathrm{K}}$, onde $\mathrm{j}_{\mathrm{L}} \mathrm{j}_{\mathrm{j}}, \mathrm{j}_{\mathrm{K}}$ são os números dos nós que compõem o elemento, nas suas respectivas extremidades I, J e K. Os eixos locais dos elementos "1, 2 e 3" seguem a regra da mão direita e são assim definidos: V3 tem sempre sua direção normal ao plano do elemento da casca, V1 vai do ponto central do lado J-K do triângulo para a sua extremidade I e o produto vetorial de V3 por V1 define a direção e o sentido de V2. 


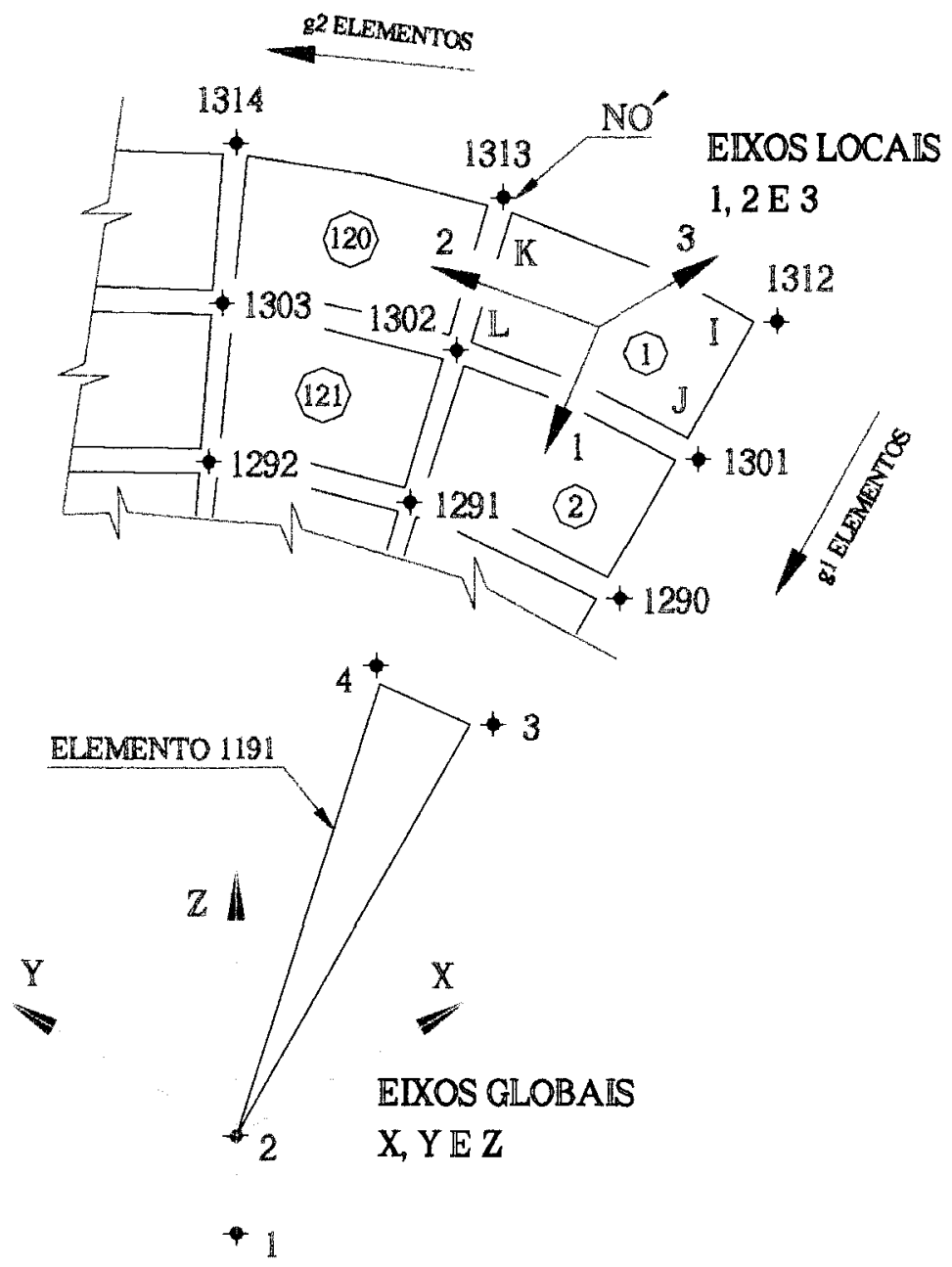

FIGURA 28 - Esquema da geração dos elementos

- $6^{0}$ BLOCO : Ação dos carregamentos na estrutura POTENTIAL

$213221 \mathrm{~W}=1 \mathrm{E}-06,1546.4102$

A casca cônica está sendo carregada, do nó 2 ao 1322 , com incremento de 1 , com pressão hidrostática causada por uma coluna de água de peso específico $\gamma=$ $1.10^{-6}\left[\mathrm{ton} / \mathrm{cm}^{3}\right]$ e altura de $[1200+(600 \cdot \tan \theta \mathrm{r})]$. Com $\theta \mathrm{r}=30$ [graus $]$ obtem-se $1546,4102[\mathrm{~cm}]$

Esta definida a entrada de dados com os comandos necessários para que o programa SAP90 possa resolver a casca cônica de revolução, sob a ação de pressão hidrostática, vinculada continuamente na sua borda superior primeiramente por um engastamento $(\mathrm{R}=1,1,1,1,1,1)$ e depois por apoio articulado fixo $(\mathrm{R}=1,1,1,0,0,0)$ 


\section{ESTRUTURA "TÍPICA" ANALISADA - COMENTÁRIOS GERAIS}

Relembrando o objetivo de utilizar a teoria de estruturas em casca de revolução e programa de análise de estruturas por elementos finitos no cálculo de cascas cilíndrica e cônica de um equipamento com dimensões "típicas" utilizadas na área de processos da indústria sucro-alcooleira, apresenta-se as suas características gerais adotadas

- Descrição : Reservatório metálico elevado com corpo cilíndrico e fundo e teto cônicos sustentado por estrutura metálica.

- Material do reservatório : Aço Carbono ASTM A283C

Módulo de elasticidade $\mathrm{E}=2100\left[\mathrm{ton} / \mathrm{cm}^{2}\right]$

Coeficiênte de Poisson $v=0,3$

Tensão de ruptura (ASME) 3,87 [ton $\left./ \mathrm{cm}^{2}\right]$

Tensão de escoamento (ASME) 2,11 [ton $\left./ \mathrm{cm}^{2}\right]$

Peso específico $7,85\left[\right.$ ton $\left./ \mathrm{m}^{3}\right]$

- Produto armazenado

: Água

Peso específico $1,0\left[\mathrm{ton} / \mathrm{m}^{3}\right]$

- Diâmetro do corpo cilíndrico

$1200,0[\mathrm{~cm}]$

- Altura do corpo cilíndrico $1200,0[\mathrm{~cm}]$

- Espessura do corpo cilíndrico (constante) $1,0[\mathrm{~cm}]$

- Inclinação do fundo cônico com a horizontal (ângulo $\theta r$ )

$$
\begin{array}{ll}
-1^{\circ} \text { modelo } & : 30 \text { [graus] } \\
-2^{\circ} \text { modelo } & : 45 \text { [graus] } \\
-3^{\circ} \text { modelo } & : 60 \text { [graus] }
\end{array}
$$


- Altura do fundo cônico

$-1^{\circ}$ modelo

: $346,4[\mathrm{~cm}]$

$-2^{\circ}$ modelo

$600,0[\mathrm{~cm}]$

$-3^{\circ}$ modelo

$1039,2[\mathrm{~cm}]$

- Espessura do fundo cônico (constante)

$: 1,0[\mathrm{~cm}]$

- Inclinação do teto cônico com a horizontal

: 15 [graus]

- Altura do teto cônico

$160,0[\mathrm{~cm}]$

- Espessura do teto cônico (constante)

$: 0,8[\mathrm{~cm}]$

- Volume de produto armazenado $\quad-1^{\circ}$ modelo

$1500\left[\mathrm{~m}^{3}\right]$

$-2^{\circ}$ modelo

$1600\left[\mathrm{~m}^{3}\right]$

$-3^{\circ}$ modelo

$1750\left[\mathrm{~m}^{3}\right]$

- Peso do produto armazenado

$-1^{\circ}$ modelo

$: 1500$ [ton]

$-2^{\circ}$ modelo

1600 [ton]

$-3^{\circ}$ modelo

1750 [ton]

- Peso próprio estimado somente para o reservatório

- Teto cônico

7,4 [ton]

- Corpo cilíndrico

: 36,0 [ton]

- Fundo cônico

$-1^{\circ}$ modelo

$: 10,3$ [ton]

$-2^{\circ}$ modelo

13,0 [ton]

$-3^{\circ}$ modelo

$18,0[$ ton $]$

- Peso próprio total

$-1^{\circ}$ modelo

53,7 [ton]

$-2^{\circ}$ modelo

56,4 [ton]

$-3^{0}$ modelo

61,4 [ton]

- Ambiente de trabalho

: aberto ao tempo

Foi considerado, portanto, um reservatório com três ângulos diferentes para o seu fundo cônico com a finalidade de testar as expressões teóricas aproximadas.

Observar que o ângulo de 60 graus com a horizontal, na prática, dificilmente seria utilizado no projeto de um reservatório para produto líquido, visto que estes não necessitam de ângulos acentuados para o seu escoamento, e também por esta inclinação do fundo exigir altura elevada para sua estrutura de sustentação. 


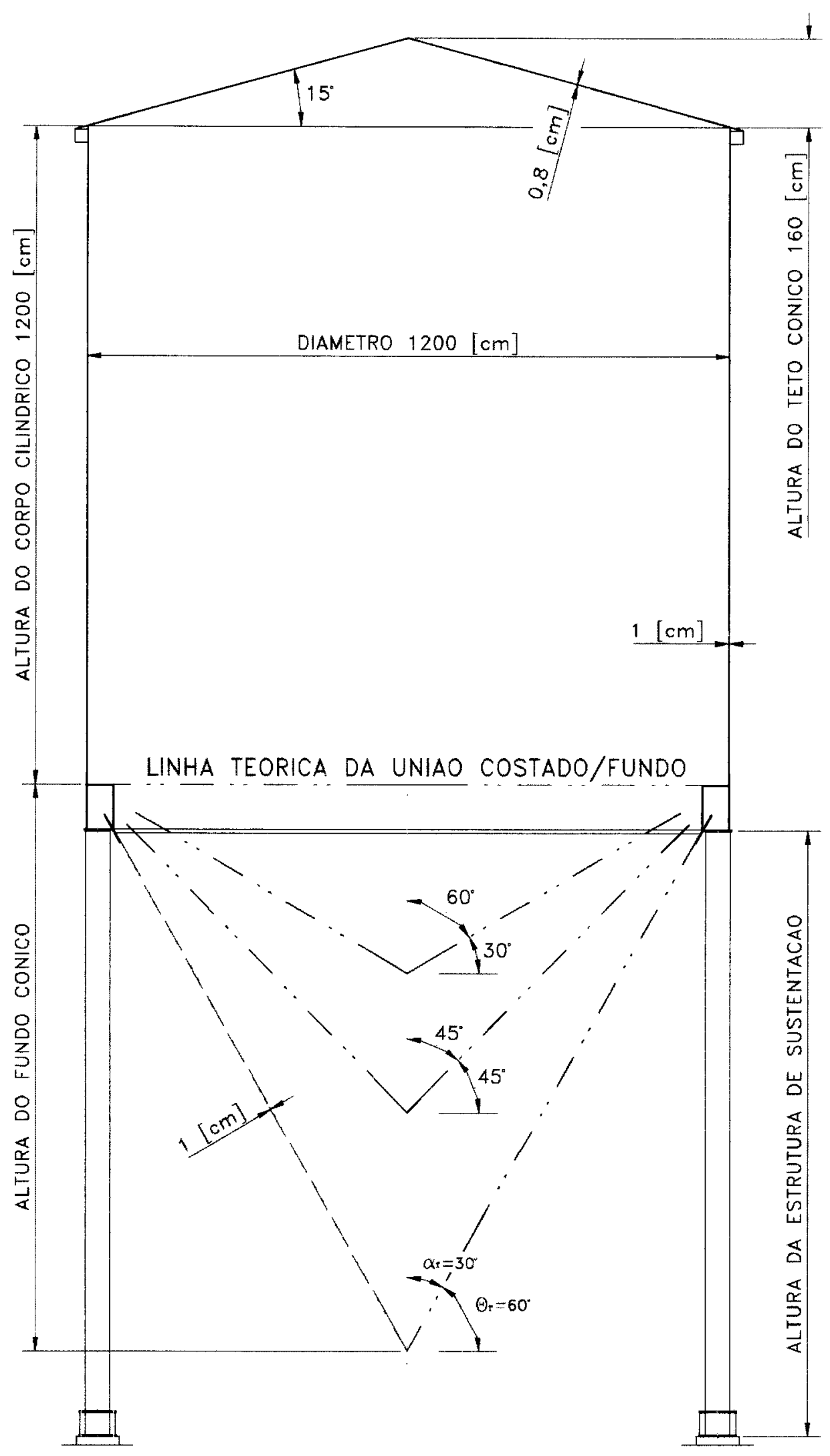

FIGURA 29 - Esquema do reservatório metálico elevado analisado com fundo cônico a 30,45 e 60 [graus] com a horizontal 
Os carregamentos de peso próprio do equipamento, sobrecarga no teto, ação do vento e ações dinâmicas não foram considerados neste trabalho.

$\mathrm{O}$ peso próprio estimado para o reservatório, pelo fato de representar aproximadamente $3,5 \%$ do peso total do mesmo cheio de produto, foi desconsiderado em favor da agilização do desenvolvimento matemático das equações teóricas. Sua consideração seria preciosismo, visto que as hipóteses fundamentais e de projeto simulam o comportamento real da estrutura de forma aproximada.

Se considerada sobrecarga de $60\left[\mathrm{kgf} / \mathrm{m}^{2}\right]$ no teto cônico, conforme recomenda a Norma Brasileira "NB 89/1978 - Tanques soldados para armazenamento de petróleo e derivados" (1978a), a tensão de compressão atuante ao longo do meridiano da casca cilíndrica de diâmetro $\mathrm{D}=1200,0[\mathrm{~cm}]$ e espessura $\mathrm{t}=1,0[\mathrm{~cm}]$ atinge o seu valor máximo na borda inferior da mesma e vale

$$
\sigma 1 \mathrm{~s}=-\frac{\mathrm{p} \cdot \mathrm{D}}{4 \cdot 10^{7} \cdot \mathrm{t}}=-0,0018\left[\mathrm{ton} / \mathrm{cm}^{2}\right] \text { que é desprezível }
$$

O efeito da ação de arrasto do vento na estrutura global, para o reservatório cheio de produto, é pouco significativo em termos de tensões que gera no corpo cilíndrico do mesmo. Utilizando a Norma Brasileira "NBR 6123/1988 - Forças devidas ao vento em edificações" (1988) fez-se as seguintes considerações rápidas

- Altura da estrutura de sustentação do reservatório $: 1200,0[\mathrm{~cm}]$

- Altura do reservatório acima da estrutura de sustentação : 1360,0 [cm]

- Altura total até o topo do teto do reservatório $2560,0[\mathrm{~cm}]$

- Velocidade básica do vento $\mathrm{V}_{\mathrm{o}}=40[\mathrm{~m} / \mathrm{s}]$

- Fator topográfico (terreno plano, fracamente acidentado) : $S_{1}=1,0$

- Fator rugosidade do terreno (Categoria III / Classe B) variável com a cota $Z$ em $[\mathrm{m}]$ acima do terreno : $\mathrm{S}_{23 \mathrm{~B}}(Z)$

- Fator estatístico (terreno plano, fracamente acidentado) $\quad: S_{3}=1,0$

- Velocidade característica do vento variável com a $\operatorname{cota} Z$ em [m] acima do terreno $\mathrm{Vk}_{3 \mathrm{~B}}(\mathrm{Z})[\mathrm{m} / \mathrm{s}]$

- Pressão dinâmica do vento variável com a $\operatorname{cota} \mathrm{Z} \mathrm{em} \mathrm{[m]} \mathrm{acima} \mathrm{do} \mathrm{terreno}$ : $\mathrm{q}_{3 \mathrm{~B}}(\mathrm{Z})\left[\mathrm{kgf} / \mathrm{m}^{2}\right]$ 
- Coeficiênte de arrasto (NBR TABELA $10 /$ acabamento liso)

- Pressão efetiva do vento variável com a cota $Z$ em [m] acima do terreno : $\mathrm{p}_{3 \mathrm{~B}}(\mathrm{Z})\left[\mathrm{kgf} / \mathrm{m}^{2}\right]$

- Forças de arrasto atuantes em 4 pontos a altura $\mathrm{Z}_{\mathrm{f}}(\mathrm{Z})$ em $[\mathrm{m}]$ do costado $: F_{3 B}(Z)[\mathrm{kgf}]$

\begin{tabular}{|c|r|r|r|r|}
\hline $\begin{array}{c}Z \\
{[\mathrm{~m}]}\end{array}$ & $\mathrm{S}_{23 \mathrm{~B}}(\mathrm{Z})$ & $\begin{array}{c}\mathrm{Vk}_{3 \mathrm{~B}}(\mathrm{Z}) \\
{[\mathrm{m} / \mathrm{s}]}\end{array}$ & $\begin{array}{c}\mathrm{q}_{3 \mathrm{~B}}(\mathrm{Z}) \\
{\left[\mathrm{kgf} / \mathrm{m}^{2}\right]}\end{array}$ & $\begin{array}{c}\mathrm{p}_{3 \mathrm{~B}}(\mathrm{Z}) \\
{\left[\mathrm{kgf} / \mathrm{m}^{2}\right]}\end{array}$ \\
\hline 5,12 & 0,86 & 34 & 74 & 37 \\
\hline 10,24 & 0,92 & 37 & 85 & 43 \\
\hline 15,36 & 0,96 & 39 & 93 & 46 \\
\hline 20,48 & 0,99 & 40 & 99 & 49 \\
\hline 25,60 & 1,02 & 41 & 103 & 52 \\
\hline
\end{tabular}

\begin{tabular}{|r|r|}
\hline $\begin{array}{c}\mathrm{z}_{\mathrm{f}}(\mathrm{Z}) \\
{[\mathrm{m}]}\end{array}$ & $\begin{array}{c}\mathrm{F}_{3 \mathrm{~B}}(\mathrm{Z}) \\
{[\mathrm{kgf}]}\end{array}$ \\
\hline 1,5 & 1663 \\
\hline 4,5 & 1728 \\
\hline 7,5 & 1785 \\
\hline 10,5 & 1836 \\
\hline
\end{tabular}

- Momento na posição da união costado/fundo para forças de arrasto atuantes no costado : $\mathrm{Mv}=4293,5$ [ton. $\mathrm{cm}]$

Com este valor de momento agindo na base do corpo cilíndrico pode-se encontrar de forma "simplista" a tensão de flexão máxima atuante no meridiano da casca cilindrica

$$
\sigma l v=+/-\frac{4 \cdot M v}{\pi \cdot t \cdot D^{2}}=+/-0,0038\left[\text { ton } / \mathrm{cm}^{2}\right] \text { que também é desprezivel. }
$$

É importante observar que a ação localizada do vento na casca, principalmente quando esta se encontra totalmente vazia, merece um estudo específico devido a sua complexidade, mas não faz parte do escopo deste trabalho.

Ações dinâmicas não foram consideradas pois os processos químicos que normalmente se utilizam destes equipamentos são na sua maioria praticamente estáticos (fermentação, decantação, etc).

Esta rápida análise das ações atuantes no reservatório, foi feita com o objetivo de justificar a opção pelo desenvolvimento do cálculo estrutural, tanto para as equações teóricas como para o método dos elementos finitos, apenas para a ação do carregamento do produto armazenado até a altura do topo do corpo cilíndrico. 


\section{CALCULANDO A ESTRUTURA "TÍPICA"}

\subsection{CONSIDERAÇÕES GERAIS}

Partindo dos dados apresentados no capítulo anterior fez-se o cálculo das cascas de revolução cilíndrica e cônica através das equações teóricas e do método dos elementos finitos (MEF).

Foi considerado como sendo importante para este trabalho calcular os seguintes parâmetros, tanto para a casca cilíndrica como para a cônica :

- comprimento ao longo do meridiano da casca necessário para o amortecimento das tensões de flexão causadas pela "perturbação de borda" em $[\mathrm{cm}]$ :

$\begin{array}{ll}\text { - cilíndrica } & : \lambda \\ \text { - cônica } & : \lambda_{\text {cone }}\end{array}$

- deslocamentos radiais ao longo do meridiano da superficie média da casca $\mathrm{em}[\mathrm{cm}]$ :

$\begin{array}{ll}\text { - cilindrica engastada } & : W e(z) \\ \text { - cilindrica apoiada fixa } & : W a(z) \\ \text { - cônica engastada } & : \xi \mathrm{e}(\mathrm{z}) \\ \text { - cônica apoiada fixa } & : \xi \mathrm{g}(\mathrm{z})\end{array}$

- rotações ao longo do meridiano da superficie média da casca em [radianos]:

- cilíndrica e cônica engastadas : : $\phi \mathrm{e}(\mathrm{z})$

- cilíndrica e cônica apoiada fixa $\quad: \phi a(z)$

- momentos solicitantes ao longo dos paralelos da superficie média da casca em $[$ ton $. \mathrm{cm} / \mathrm{cm}]$ : 
- cilíndrica e cônica engastadas $\quad:$ Mle(z)

- cilíndrica e cônica apoiada fixa $:$ Mla(z)

- momentos solicitantes ao longo dos meridianos da superficie média da casca em $[$ ton $\mathrm{cm} / \mathrm{cm}]$

- cilíndrica e cônica engastadas : : M2e(z)

- cilíndrica e cônica apoiada fixa : $\mathrm{M} 2 \mathrm{a}(\mathrm{z})$

- tensões atuantes ao longo dos meridianos da superficie interna da casca em $\left[\mathrm{ton} / \mathrm{cm}^{2}\right]$ :

- cilíndrica e cônica engastadas $\quad: \sigma$ lein(z)

- cilíndrica e cônica apoiada fixa : $\sigma$ lain(z)

- tensões atuantes ao longo dos meridianos da superficie externa da casca em [ton $\left./ \mathrm{cm}^{2}\right]$ :

- cilíndrica e cônica engastadas : $\sigma$ leex $(\mathrm{z})$

- cilíndrica e cônica apoiada fixa $: \sigma$ laex(z)

- tensões atuantes ao longo dos paralelos da superfície interna da casca em $\left[\mathrm{ton} / \mathrm{cm}^{2}\right]$ :

- cilíndrica e cônica engastadas $\quad: \sigma 2 \operatorname{ein}(\mathrm{z})$

- cilíndrica e cônica apoiada fixa : $\sigma 2 \operatorname{ain}(\mathrm{z})$

- tensões atuantes ao longo dos paralelos da superfície externa da casca em $\left[\mathrm{ton} / \mathrm{cm}^{2}\right]$ :

- cilíndrica e cônica engastadas : $: \sigma 2 \operatorname{eex}(\mathrm{z})$

- cilíndrica e cônica apoiada fixa : $\sigma 2 \mathrm{aex}(\mathrm{z})$

- tensões teóricas de membrana atuantes ao longo dos meridianos da superfície média da casca em [ton $\left./ \mathrm{cm}^{2}\right]$ :

- cilíndrica e cônica $\quad: \sigma \operatorname{lm}(\mathrm{z})$

- tensões teóricas de membrana atuantes ao longo dos paralelos da superficie média da casca em $\left[\right.$ ton $\left./ \mathrm{cm}^{2}\right]$ :

- cilíndrica e cônica $\quad: \sigma 2 \mathrm{~m}(\mathrm{z})$ 
Para maior agilidade no cálculo pelas equações teóricas, utilizou-se um programa de planilha eletrônica, no qual codificou-se as equações obtendo os valores dos parâmetros citados.

Pelo método dos elementos finitos, obteve-se do programa SAP90 os resultados dos parâmetros referentes aos deslocamentos radiais, rotações, momentos solicitantes e tensões atuantes para a ação da pressão hidrostática nas cascas.

Os resultados para momentos e tensões tiveram que ser trabalhados, pois para cada nó da estrutura desmembrada, ou seja, para cada nó dos elementos finitos da casca, são apresentados quatro valores do parâmetro (momento ou tensão). Para se obter o momento ou a tensão em um nó do elemento deve-se simplesmente fazer a média aritmética dos quatros valores apresentados pelo programa para aquele nó

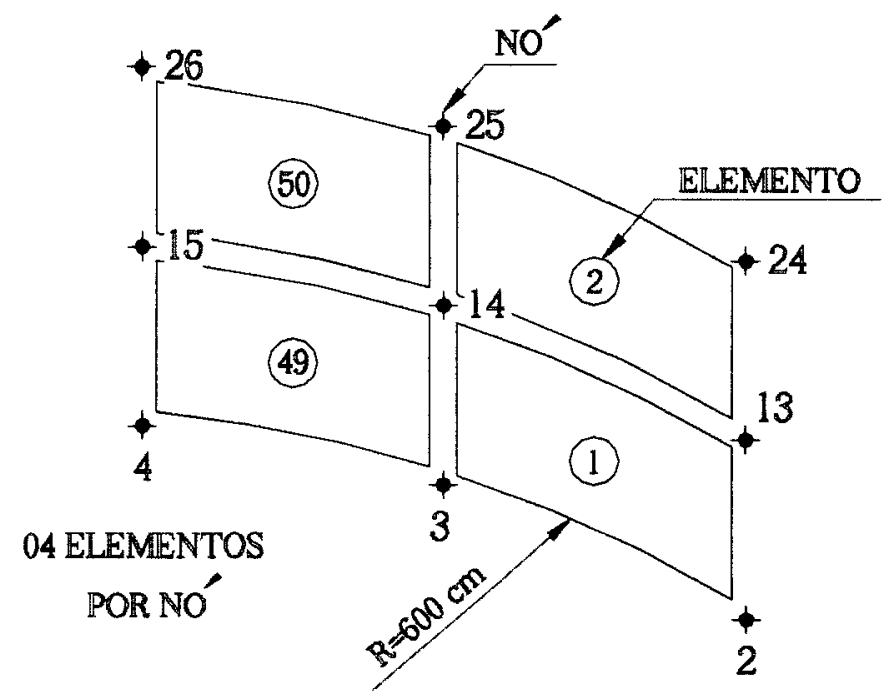

FIGURA 30 - Esquema para compreensão da existência de quatro parâmetros por nó

A casca cilíndrica foi calculada até uma altura de 240 [cm] e carregada com $1200[\mathrm{~cm}]$ de coluna de água, como já citado no capítulo 3

A casca cônica foi calculada com três diferentes ângulos de inclinação com a horizontal de 30, 45 e 60 graus. Portanto para casca cônica tem-se três modelos de estrutura calculada, carregadas com uma coluna de água de 1200 [cm] mais a altura do cone.

Os resultados obtidos através das equações teóricas aproximadas e por elementos finitos serão tabelados lado a lado para que possam ser observados 
comparativamente. Os valores foram tabelados em função da coordenada " $z$ " na direção do eixo vertical, medida para a casca cilíndrica a partir da borda vinculada e, para casca cônica a partir de seu bico, coincidente com o eixo de rotação da casca. Apesar do cálculo ter sido efetuado para toda extensão das cascas, as tabelas serão apresentadas até a coordenada " $\mathrm{z}$ " que corresponda aproximadamente ao comprimento $\lambda$ (ou $\lambda_{\text {cone }}$ ). Deste modo, visualiza-se a região das cascas influenciada pelas tensões de flexão causadas pela "perturbação de borda", além de deixar este capítulo menos cansativo ao leitor.

Os gráficos traçados com os resultados obtidos pelos dois métodos também serão apresentados lado a lado para permitir uma melhor visualização das curvas obtidas.

\subsection{CALCULANDO E OBTENDO RESUltados PARA A CASCA CILÍNDRICA}

Através das equações teóricas do capítulo 2, e com os dados abaixo :

\begin{tabular}{|c|c|c|c|c|c|c|c|}
\hline $\begin{array}{c}\mathrm{E} \\
{\left[\text { ton } / \mathrm{cm}^{2}\right]}\end{array}$ & $\begin{array}{c}v \\
(\mathrm{Aço})\end{array}$ & $\begin{array}{c}\gamma \\
{\left[\text { ton } / \mathrm{cm}^{3}\right]}\end{array}$ & $\begin{array}{c}\mathrm{D} \\
{[\mathrm{cm}]}\end{array}$ & $\begin{array}{c}\mathrm{R} \\
{[\mathrm{cm}]}\end{array}$ & $\begin{array}{c}\mathrm{H} \\
{[\mathrm{cm}]}\end{array}$ & $\begin{array}{c}\mathrm{h} \\
{[\mathrm{cm}]}\end{array}$ & $\begin{array}{c}\mathrm{t} \\
{[\mathrm{cm}]}\end{array}$ \\
\hline 2100 & 0,3 & $1,00 \mathrm{E}-06$ & 1200 & 600 & 1200 & 1200 & 1 \\
\hline
\end{tabular}

Encontram-se

\begin{tabular}{|c|c|c|c|c|c|c|}
\hline $\begin{array}{c}\beta \\
{\left[\text { ton } / \mathrm{cm}^{3}\right]}\end{array}$ & $\begin{array}{c}\mathrm{B} \\
{[\text { ton/cm] }}\end{array}$ & $\begin{array}{c}\alpha \\
{\left[\mathrm{cm}^{-1}\right]}\end{array}$ & $\begin{array}{c}\text { wh } \\
{\left[\mathrm{cm}^{2} / \text { ton }\right]}\end{array}$ & $\begin{array}{c}\text { wm } \\
{[\mathrm{cm} / \text { ton }]}\end{array}$ & $\begin{array}{c}\phi \mathrm{h} \\
{[\mathrm{cm} / \text { ton }]}\end{array}$ & $\begin{array}{c}\phi \mathrm{m} \\
{\left[\text { ton }^{-1}\right]}\end{array}$ \\
\hline 0,0058 & 192,3077 & 0,0525 & 17,9920 & 0,9442 & 0,9442 & 0,0991 \\
\hline
\end{tabular}

\begin{tabular}{|c|c|c|}
\hline $\begin{array}{c}\text { Mce } \\
\text { [ton } \mathrm{cm} / \mathrm{cm}]\end{array}$ & $\begin{array}{c}\text { Hce } \\
{[\text { ton } / \mathrm{cm}]}\end{array}$ & $\begin{array}{c}\text { Hca } \\
{[\text { ton } / \mathrm{cm}]}\end{array}$ \\
\hline 0,2144 & $-0,0227$ & $-0,0114$ \\
\hline
\end{tabular}


E para o comprimento ao longo do meridiano da casca necessário para o amortecimento das tensões de flexão causadas pela "perturbação de borda" medido a partir do vínculo :

$$
-\lambda=119,73[\mathrm{~cm}]
$$

Portanto a tabela de valores para a casca cilíndrica será apresentada até a coordenada " $\mathrm{z}$ " adotada como igual a $125[\mathrm{~cm}]$.

\subsubsection{CASCA CILÍNDRICA COM BORDA INFERIOR ENGASTADA}

Para os deslocamentos radiais e rotações ao longo do meridiano da superficie média da casca cilíndrica encontrou-se :

TABELA 1 - Valores dos deslocamentos radiais $\mathrm{We}(\mathrm{z})$ e rotações $\phi \mathrm{e}(\mathrm{z})$

\begin{tabular}{|c|r|r|r|}
\cline { 2 - 3 } \multicolumn{1}{c|}{} & \multicolumn{2}{c|}{\begin{tabular}{c}
\multicolumn{1}{c}{ We(z) } \\
{$[\mathrm{cm}]$}
\end{tabular}} & \multicolumn{1}{c}{} \\
\hline $\begin{array}{c}z \\
{[\mathrm{~cm}]}\end{array}$ & Teoria & $\begin{array}{c}\text { Elementos } \\
\text { Finitos }\end{array}$ & Diferença \\
\hline 0,00 & 0,0000 & 0,0000 & 0,0000 \\
\hline 5,00 & 0,0116 & 0,0115 & 0,0001 \\
\hline 10,00 & 0,0386 & 0,0383 & 0,0003 \\
\hline 15,00 & 0,0718 & 0,0713 & 0,0005 \\
\hline 20,00 & 0,1050 & 0,1044 & $\mathbf{0 , 0 0 0 6}$ \\
\hline 25,00 & 0,1345 & 0,1340 & 0,0006 \\
\hline 30,00 & 0,1588 & 0,1582 & 0,0006 \\
\hline 35,00 & 0,1772 & 0,1767 & 0,0005 \\
\hline 40,00 & 0,1901 & 0,1897 & 0,0004 \\
\hline 45,00 & 0,1984 & 0,1980 & 0,0004 \\
\hline 50,00 & 0,2028 & 0,2025 & 0,0003 \\
\hline 55,00 & 0,2045 & 0,2042 & 0,0003 \\
\hline 60,00 & 0,2043 & 0,2040 & 0,0003 \\
\hline 65,00 & 0,2029 & 0,2026 & 0,0003 \\
\hline 70,00 & 0,2008 & 0,2004 & 0,0004 \\
\hline 75,00 & 0,1985 & 0,1981 & 0,0004 \\
\hline 80,00 & 0,1962 & 0,1957 & 0,0005 \\
\hline 85,00 & 0,1940 & 0,1935 & 0,0005 \\
\hline 90,00 & 0,1921 & 0,1915 & 0,0005 \\
\hline 95,00 & 0,1904 & 0,1898 & 0,0006 \\
\hline 100,00 & 0,1889 & 0,1884 & 0,0006 \\
\hline
\end{tabular}

\begin{tabular}{|c|c|c|}
\hline \multicolumn{2}{|c|}{$\begin{array}{c}\phi \mathrm{e}(\mathrm{z}) \\
\text { [radianos] }\end{array}$} & \\
\hline Teoria & $\begin{array}{c}\text { Elementos } \\
\text { Finitos }\end{array}$ & Diferença \\
\hline 0,0000 & 0,0000 & 0,0000 \\
\hline 0,0042 & 0,0042 & 0,0000 \\
\hline 0,0063 & 0,0062 & 0,0000 \\
\hline 0,0068 & 0,0068 & 0,0000 \\
\hline 0,0064 & 0,0064 & 0,0000 \\
\hline 0,0054 & 0,0054 & 0,0000 \\
\hline 0,0043 & 0,0043 & 0,0000 \\
\hline 0,0031 & 0,0031 & 0,0000 \\
\hline 0,0021 & 0,0021 & 0,0000 \\
\hline 0,0012 & 0,0012 & 0,0000 \\
\hline 0,0006 & 0,0006 & 0,0000 \\
\hline 0,0001 & 0,0001 & 0,0000 \\
\hline$-0,0002$ & $-0,0002$ & 0,0000 \\
\hline$-0,0004$ & $-0,0004$ & 0,0000 \\
\hline$-0,0005$ & $-0,0005$ & 0,0000 \\
\hline$-0,0005$ & $-0,0005$ & 0,0000 \\
\hline$-0,0005$ & $-0,0005$ & 0,0000 \\
\hline$-0,0004$ & $-0,0004$ & 0,0000 \\
\hline$-0,0004$ & $-0,0004$ & 0,0000 \\
\hline$-0,0003$ & $-0,0003$ & 0,0000 \\
\hline$-0,0003$ & $-0,0003$ & 0,0000 \\
\hline
\end{tabular}




\begin{tabular}{|c|r|r|r|}
\cline { 2 - 3 } \multicolumn{1}{c|}{} & \multicolumn{2}{c|}{\begin{tabular}{c}
\multicolumn{1}{c}{} \\
$\mathrm{cm}(\mathrm{z})$
\end{tabular}} & \multicolumn{1}{c}{} \\
\hline $\begin{array}{c}\mathrm{z} \\
{[\mathrm{cm}]}\end{array}$ & Teoria & $\begin{array}{c}\text { Elementos } \\
\text { Finitos }\end{array}$ & Diferença \\
\hline 105,00 & 0,1877 & 0,1871 & 0,0006 \\
\hline 110,00 & 0,1866 & 0,1860 & 0,0006 \\
\hline 115,00 & 0,1856 & 0,1850 & 0,0006 \\
\hline 120,00 & 0,1848 & 0,1842 & 0,0006 \\
\hline 125,00 & 0,1839 & 0,1833 & 0,0006 \\
\hline
\end{tabular}

\begin{tabular}{|c|r|r|}
\hline \multicolumn{2}{|c|}{\begin{tabular}{c}
\multicolumn{1}{c}{$\phi(z)$} \\
radianos
\end{tabular}} & \\
\hline Teoria & $\begin{array}{c}\text { Elementos } \\
\text { Finitos }\end{array}$ & Diferença \\
\hline$-0,0002$ & $-0,0002$ & 0,0000 \\
\hline$-0,0002$ & $-0,0002$ & 0,0000 \\
\hline$-0,0002$ & $-0,0002$ & 0,0000 \\
\hline$-0,0002$ & $-0,0002$ & 0,0000 \\
\hline$-0,0002$ & $-0,0002$ & 0,0000 \\
\hline
\end{tabular}
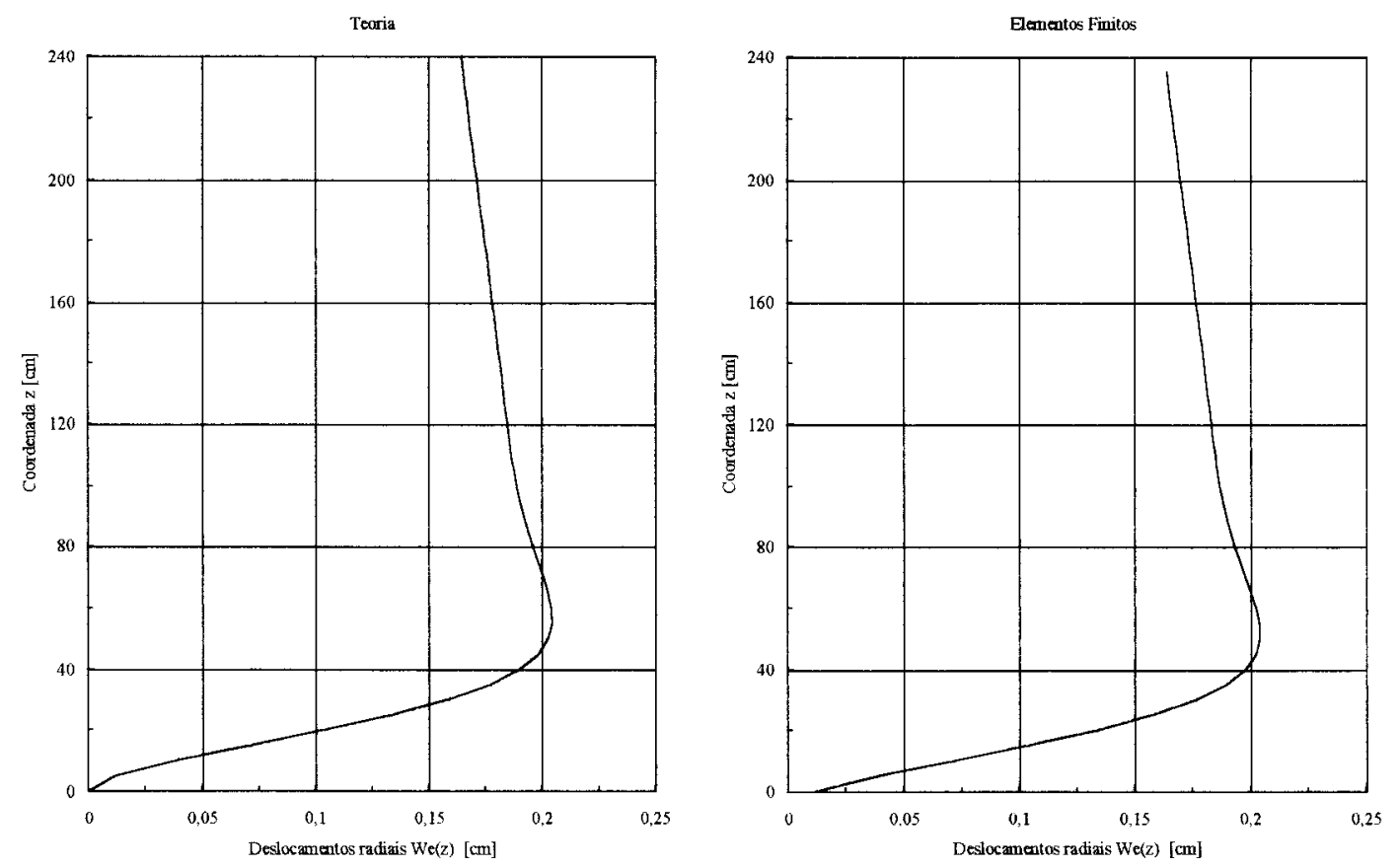

FIGURA 31 - Gráficos para os valores dos deslocamentos radiais $\mathrm{We}(\mathrm{z})$ ao longo do meridiano da superficie média da casca cilíndrica

A coluna "Diferença" da tabela apresenta os valores das diferenças absolutas entre os parâmetros obtidos pelas equações da teoria aproximada e os obtidos pelo cálculo através do método dos elementos finitos. 

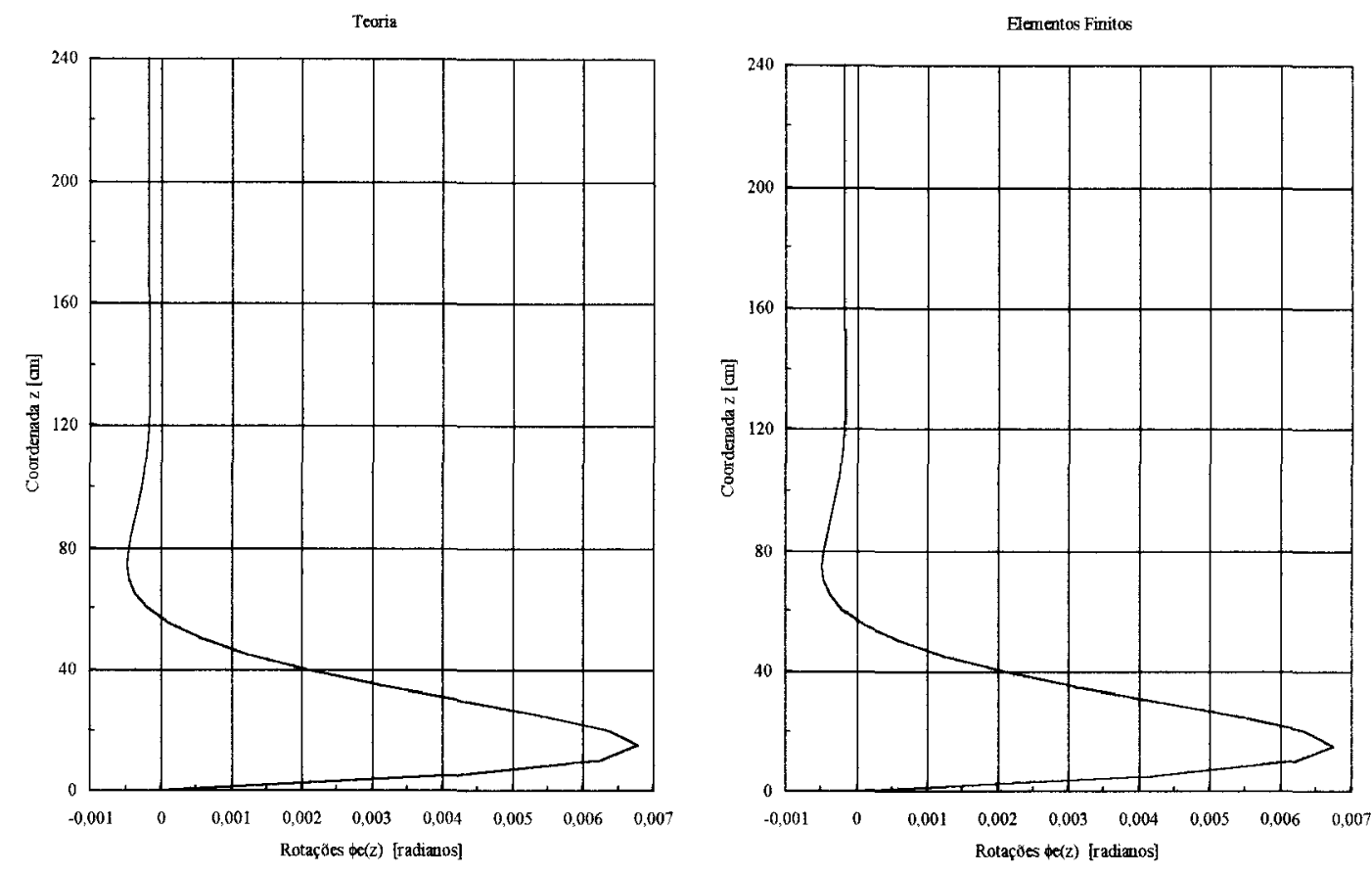

FIGURA 32 - Gráficos para os valores das rotações $\phi \mathrm{e}(\mathrm{z})$ ao longo do meridiano da superficie média da casca cilíndrica

Os momentos solicitantes ao longo dos paralelos e dos meridianos da superficie média da casca cilíndrica e suas correspondentes diferenças absolutas foram

TABELA 2 - Valores dos momentos ao longo dos paralelos $\mathrm{M} 1 \mathrm{e}(\mathrm{z})$ e dos meridianos M2e(z)

\begin{tabular}{|c|c|c|c|}
\hline & \multicolumn{2}{|c|}{$\begin{array}{c}\mathrm{Mle}(\mathrm{z}) \\
{[\text { ton. } \mathrm{cm} / \mathrm{cm}]}\end{array}$} & \\
\hline $\begin{array}{c}\mathrm{z} \\
{[\mathrm{cm}]}\end{array}$ & Teoria & $\begin{array}{c}\text { Elementos } \\
\text { Finitos }\end{array}$ & Diferença \\
\hline 0,00 & $-0,2144$ & $-0,2092$ & 0,0053 \\
\hline 5,00 & $-0,1158$ & $-0,1125$ & 0,0033 \\
\hline 10,00 & $-0,0452$ & $-0,0434$ & 0,0018 \\
\hline 15,00 & 0,0014 & 0,0023 & 0,0010 \\
\hline 20,00 & 0,0288 & 0,0292 & 0,0005 \\
\hline 25,00 & 0,0419 & 0,0422 & 0,0003 \\
\hline 30,00 & 0,0453 & 0,0454 & 0,0002 \\
\hline 35,00 & 0,0425 & 0,0426 & 0,0001 \\
\hline 40,00 & 0,0363 & 0,0364 & 0,0001 \\
\hline
\end{tabular}

\begin{tabular}{|r|r|r|}
\hline \multicolumn{2}{|c|}{$\begin{array}{r}\text { M2e(z) } \\
\text { [ton.cm/cm] }\end{array}$} & \\
\cline { 1 - 2 } Teoria & $\begin{array}{c}\text { Elementos } \\
\text { Finitos }\end{array}$ & Diferença \\
\hline$-0,0643$ & $-0,0627$ & $\mathbf{0 , 0 0 1 6}$ \\
\hline$-0,0347$ & $-0,0338$ & 0,0010 \\
\hline$-0,0136$ & $-0,0130$ & 0,0005 \\
\hline 0,0004 & 0,0007 & 0,0003 \\
\hline 0,0086 & 0,0088 & 0,0001 \\
\hline 0,0126 & 0,0127 & 0,0001 \\
\hline 0,0136 & 0,0136 & 0,0000 \\
\hline 0,0127 & 0,0128 & 0,0000 \\
\hline 0,0109 & 0,0109 & 0,0000 \\
\hline
\end{tabular}




\begin{tabular}{|c|c|c|c|}
\cline { 2 - 3 } \multicolumn{1}{c|}{} & \multicolumn{2}{c}{$\begin{array}{c}\text { Mle(z) } \\
\text { [ton.cm/cm] }\end{array}$} & \multicolumn{1}{c}{} \\
\hline $\begin{array}{c}z \\
{[\mathrm{~cm}]}\end{array}$ & Teoria & $\begin{array}{c}\text { Elementos } \\
\text { Finitos }\end{array}$ & Diferença \\
\hline 45,00 & 0,0288 & 0,0289 & 0,0001 \\
\hline 50,00 & 0,0213 & 0,0214 & 0,0001 \\
\hline 55,00 & 0,0146 & 0,0147 & 0,0000 \\
\hline 60,00 & 0,0091 & 0,0091 & 0,0000 \\
\hline 65,00 & 0,0049 & 0,0049 & 0,0000 \\
\hline 70,00 & 0,0019 & 0,0018 & 0,0001 \\
\hline 75,00 & $-0,0001$ & $-0,0002$ & 0,0001 \\
\hline 80,00 & $-0,0013$ & $-0,0013$ & 0,0001 \\
\hline 85,00 & $-0,0018$ & $-0,0019$ & 0,0001 \\
\hline 90,00 & $-0,0020$ & $-0,0020$ & 0,0001 \\
\hline 95,00 & $-0,0018$ & $-0,0019$ & 0,0001 \\
\hline 100,00 & $-0,0016$ & $-0,0016$ & 0,0001 \\
\hline 105,00 & $-0,0012$ & $-0,0013$ & 0,0000 \\
\hline 110,00 & $-0,0009$ & $-0,0009$ & 0,0000 \\
\hline 115,00 & $-0,0006$ & $-0,0006$ & 0,0000 \\
\hline 120,00 & $-0,0004$ & $-0,0004$ & 0,0000 \\
\hline 125,00 & $-0,0002$ & $-0,0002$ & 0,0000 \\
\hline & & & \\
\hline
\end{tabular}

\begin{tabular}{|r|r|r|}
\hline \multicolumn{2}{|c|}{$\begin{array}{r}\text { M2e(z) } \\
\text { ton.cm/cm] }\end{array}$} & \\
\cline { 1 - 2 } Teoria & $\begin{array}{c}\text { Elementos } \\
\text { Finitos }\end{array}$ & Diferença \\
\hline 0,0086 & 0,0087 & 0,0000 \\
\hline 0,0064 & 0,0064 & 0,0000 \\
\hline 0,0044 & 0,0044 & 0,0000 \\
\hline 0,0027 & 0,0027 & 0,0000 \\
\hline 0,0015 & 0,0015 & 0,0000 \\
\hline 0,0006 & 0,0005 & 0,0000 \\
\hline 0,0000 & $-0,0001$ & 0,0000 \\
\hline$-0,0004$ & $-0,0004$ & 0,0000 \\
\hline$-0,0005$ & $-0,0006$ & 0,0000 \\
\hline$-0,0006$ & $-0,0006$ & 0,0000 \\
\hline$-0,0005$ & $-0,0006$ & 0,0000 \\
\hline$-0,0005$ & $-0,0005$ & 0,0000 \\
\hline$-0,0004$ & $-0,0004$ & 0,0000 \\
\hline$-0,0003$ & $-0,0003$ & 0,0000 \\
\hline$-0,0002$ & $-0,0002$ & 0,0000 \\
\hline$-0,0001$ & $-0,0001$ & 0,0000 \\
\hline$-0,0001$ & $-0,0001$ & 0,0000 \\
\hline
\end{tabular}

Tearia

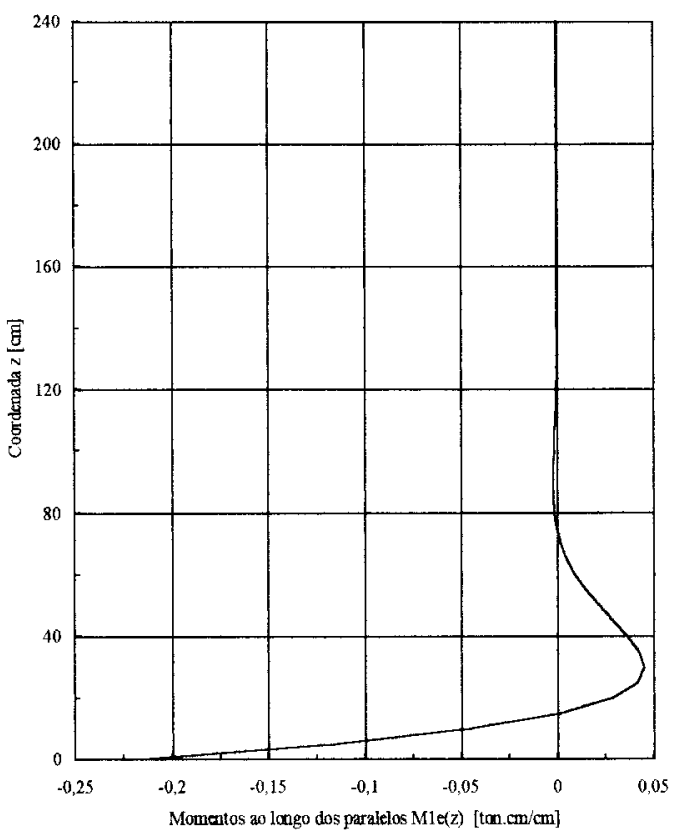

Elementos Finitos

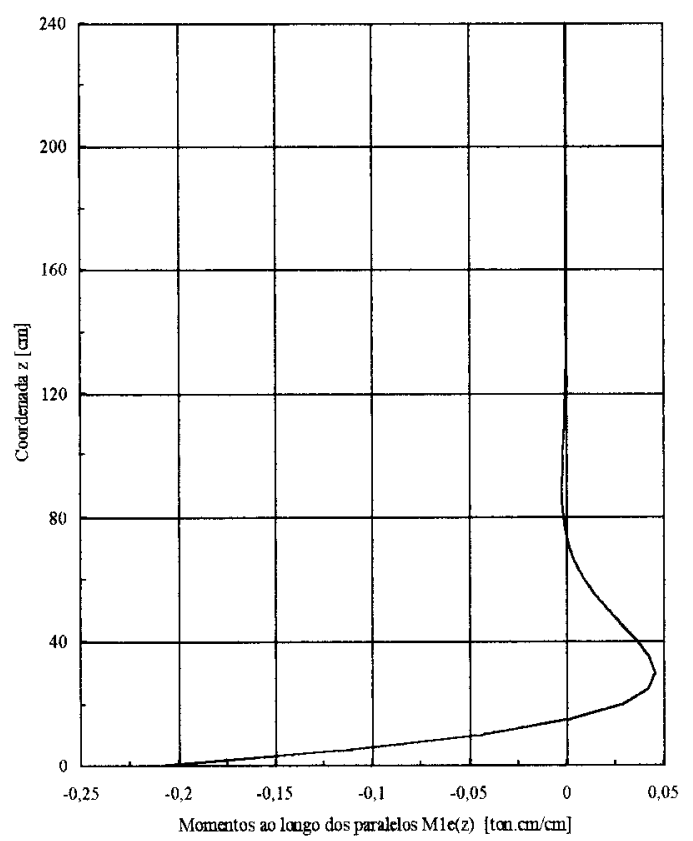

FIGURA 33 - Gráficos para os valores dos momentos ao longo dos paralelos Mle(z) da superficie média da casca cilindrica 

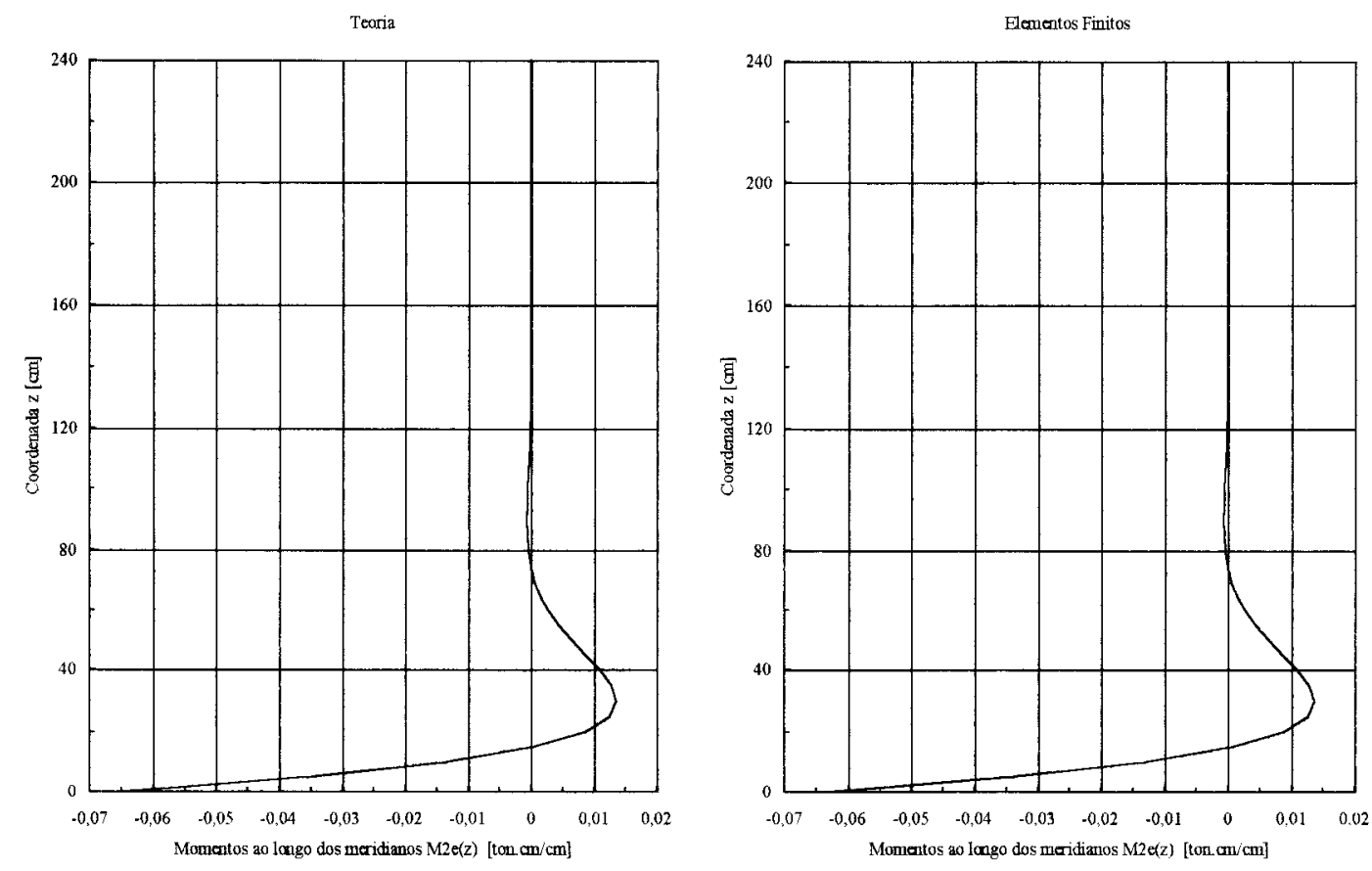

FIGURA 34 - Gráficos para os valores dos momentos ao longo dos meridianos M2e(z) da superficie média da casca cilíndrica

Para as tensões atuantes ao longo dos meridianos da superficie interna e externa da casca cilíndrica obteve-se

TABELA 3 - Valores para as tensões ao longo dos meridianos da superficie interna $\sigma$ lein(z) e externa $\sigma$ leex(z) da casca

\begin{tabular}{|c|r|r|r|}
\cline { 2 - 3 } \multicolumn{1}{c|}{} & \multicolumn{2}{c|}{\begin{tabular}{c}
\multicolumn{1}{c}{$\sigma$ ein $(\mathrm{z})$} \\
{$\left[\right.$ ton/ $\left.\mathrm{cm}^{2}\right]$}
\end{tabular}} & \multicolumn{1}{c|}{} \\
\hline $\begin{array}{c}\mathrm{z} \\
{[\mathrm{cm}]}\end{array}$ & Teoria & $\begin{array}{c}\text { Elementos } \\
\text { Finitos }\end{array}$ & Diferença \\
\hline 0,00 & 1,2865 & 1,2482 & $\mathbf{0 , 0 3 8 3}$ \\
\hline 5,00 & 0,6949 & 0,6708 & 0,0241 \\
\hline 10,00 & 0,2713 & 0,2585 & 0,0127 \\
\hline 15,00 & $-0,0081$ & $-0,0139$ & 0,0058 \\
\hline 20,00 & $-0,1726$ & $-0,1745$ & 0,0019 \\
\hline 25,00 & $-0,2516$ & $-0,2516$ & 0,0000 \\
\hline 30,00 & $-0,2717$ & $-0,2710$ & 0,0008 \\
\hline 35,00 & $-0,2549$ & $-0,2540$ & 0,0009 \\
\hline 40,00 & $-0,2179$ & $-0,2171$ & 0,0007 \\
\hline 45,00 & $-0,1729$ & $-0,1724$ & 0,0006 \\
\hline
\end{tabular}

\begin{tabular}{|r|r|r|}
\hline \multicolumn{2}{|c|}{$\begin{array}{r}\sigma l e e x(z) \\
{\left[\text { ton/cm }{ }^{2}\right]}\end{array}$} & \\
\cline { 1 - 2 } Teoria & $\begin{array}{c}\text { Elementos } \\
\text { Finitos }\end{array}$ & Diferença \\
\hline$-1,2865$ & $-1,2616$ & $\mathbf{0 , 0 2 4 9}$ \\
\hline$-0,6949$ & $-0,6796$ & 0,0153 \\
\hline$-0,2713$ & $-0,2621$ & 0,0092 \\
\hline 0,0081 & 0,0138 & 0,0057 \\
\hline 0,1726 & 0,1765 & 0,0039 \\
\hline 0,2516 & 0,2547 & 0,0030 \\
\hline 0,2717 & 0,2743 & 0,0026 \\
\hline 0,2549 & 0,2572 & 0,0023 \\
\hline 0,2179 & 0,2199 & 0,0020 \\
\hline 0,1729 & 0,1745 & 0,0016 \\
\hline
\end{tabular}




\begin{tabular}{|c|c|c|c|c|c|c|}
\hline \multirow[b]{2}{*}{$\begin{array}{c}\mathrm{z} \\
{[\mathrm{cm}]}\end{array}$} & \multicolumn{2}{|c|}{$\begin{array}{c}\sigma l \operatorname{ein}(\mathrm{z}) \\
{\left[\operatorname{ton} / \mathrm{cm}^{2}\right]}\end{array}$} & \multirow[b]{2}{*}{ Diferença } & \multicolumn{2}{|c|}{$\begin{array}{c}\sigma l e e x(z) \\
{\left[\text { ton } / \mathrm{cm}^{2}\right]}\end{array}$} & \multirow[b]{2}{*}{ Diferença } \\
\hline & Teoria & $\begin{array}{c}\text { Elementos } \\
\text { Finitos }\end{array}$ & & Teoria & $\begin{array}{c}\text { Elementos } \\
\text { Finitos }\end{array}$ & \\
\hline 50,00 & $-0,1280$ & $-0,1276$ & 0,0004 & 0,1280 & 0,1292 & 0,0012 \\
\hline 55,00 & $-0,0879$ & $-0,0875$ & 0,0004 & 0,0879 & 0,0886 & 0,0008 \\
\hline 60,00 & $-0,0548$ & $-0,0545$ & 0,0004 & 0,0548 & 0,0552 & 0,0004 \\
\hline 65,00 & $-0,0295$ & $-0,0291$ & 0,0004 & 0,0295 & 0,0295 & 0,0000 \\
\hline 70,00 & $-0,0113$ & $-0,0109$ & 0,0004 & 0,0113 & 0,0111 & 0,0003 \\
\hline 75,00 & 0,0006 & 0,0010 & 0,0004 & $-0,0006$ & $-0,0010$ & 0,0004 \\
\hline 80,00 & 0,0076 & 0,0080 & 0,0004 & $-0,0076$ & $-0,0081$ & 0,0005 \\
\hline 85,00 & 0,0109 & 0,0114 & 0,0004 & $-0,0109$ & $-0,0115$ & 0,0006 \\
\hline 90,00 & 0,0117 & 0,0121 & 0,0004 & $-0,0117$ & $-0,0123$ & 0,0005 \\
\hline 95,00 & 0,0110 & 0,0113 & 0,0003 & $-0,0110$ & $-0,0115$ & 0,0005 \\
\hline 100,00 & 0,0094 & 0,0096 & 0,0003 & $-0,0094$ & $-0,0098$ & 0,0004 \\
\hline 105,00 & 0,0074 & 0,0076 & 0,0002 & $-0,0074$ & $-0,0077$ & 0,0003 \\
\hline 110,00 & 0,0055 & 0,0056 & 0,0001 & $-0,0055$ & $-0,0057$ & 0,0002 \\
\hline 115,00 & 0,0038 & 0,0038 & 0,0001 & $-0,0038$ & $-0,0039$ & 0,0001 \\
\hline 120,00 & 0,0023 & 0,0024 & 0,0000 & $-0,0023$ & $-0,0024$ & 0,0001 \\
\hline 125,00 & 0,0012 & 0,0013 & 0,0000 & $-0,0012$ & $-0,0013$ & 0,0000 \\
\hline
\end{tabular}
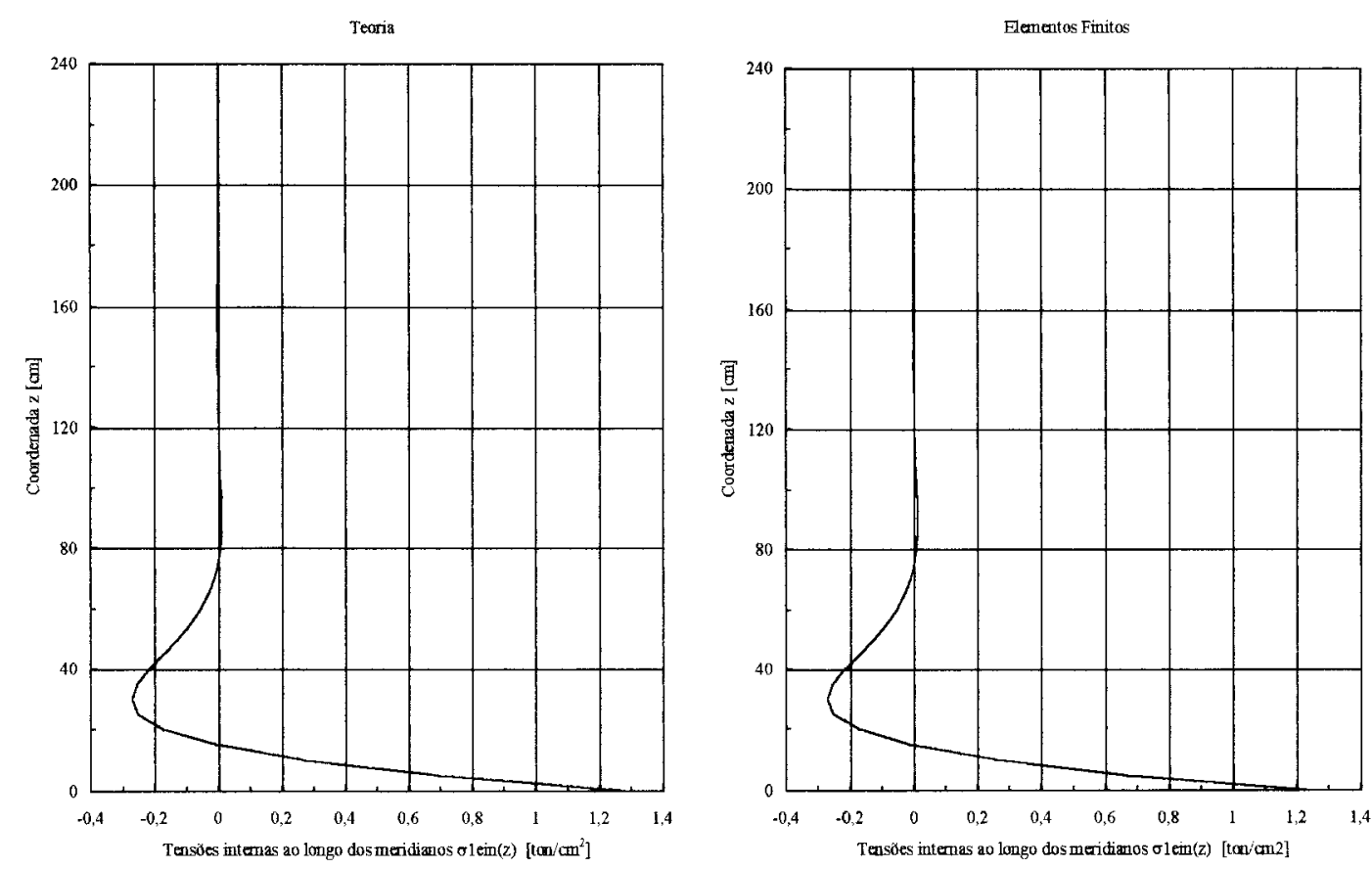

FIGURA 35 - Gráficos para os valores das tensões ao longo dos meridianos da superficie interna $\sigma$ lein $(z)$ da casca cilíndrica 

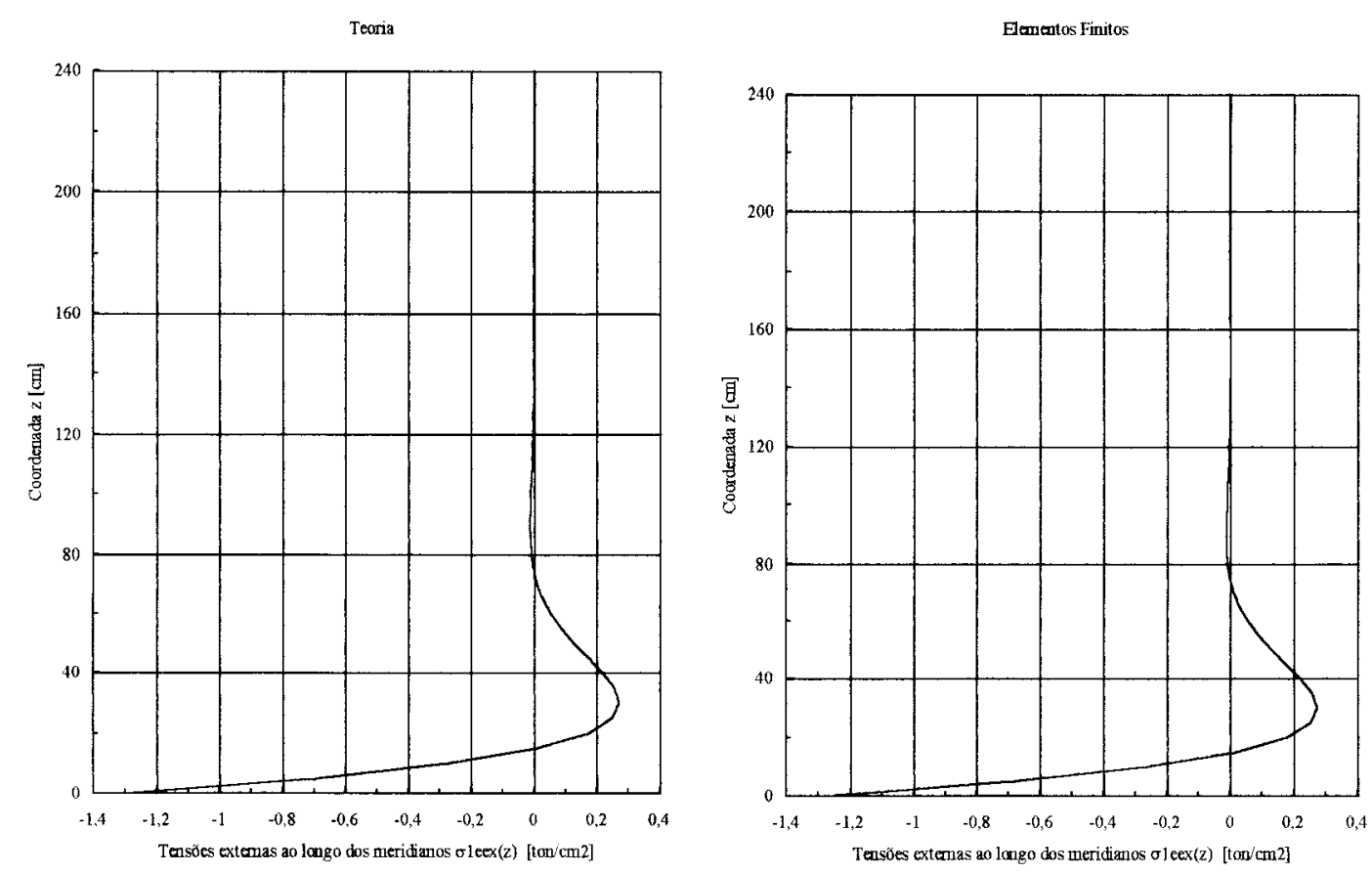

FIGURA 36 - Gráficos para os valores das tensões ao longo dos meridianos da superfície externa $\sigma$ leex $(\mathrm{z})$ da casca cilíndrica

E finalmente para as tensões atuantes ao longo dos paralelos da superficie interna e externa da casca cilíndrica encontrou-se :

TABELA 4 - Valores para as tensões ao longo dos paralelos da superficie interna $\sigma 2 \operatorname{ein}(\mathrm{z})$ e externa $\sigma 2 \operatorname{eex}(\mathrm{z})$ da casca

\begin{tabular}{|c|r|r|r|}
\cline { 2 - 3 } \multicolumn{1}{c|}{} & \multicolumn{2}{c|}{\begin{tabular}{c}
\multicolumn{1}{c}{} \\
[ton/ $\left./ \mathrm{cm}^{2}\right]$
\end{tabular}} & \multicolumn{1}{c}{} \\
\hline $\begin{array}{c}\mathrm{z} \\
{[\mathrm{cm}]}\end{array}$ & Teoria & $\begin{array}{r}\text { Elementos } \\
\text { Finitos }\end{array}$ & Diferença \\
\hline 0,00 & 0,3860 & 0,3745 & $\mathbf{0 , 0 1 1 5}$ \\
\hline 5,00 & 0,2492 & 0,2416 & 0,0076 \\
\hline 10,00 & 0,2166 & 0,2117 & 0,0049 \\
\hline 15,00 & 0,2488 & 0,2454 & 0,0034 \\
\hline 20,00 & 0,3156 & 0,3130 & 0,0026 \\
\hline 25,00 & 0,3954 & 0,3934 & 0,0021 \\
\hline 30,00 & 0,4742 & 0,4725 & 0,0017 \\
\hline 35,00 & 0,5437 & 0,5423 & 0,0014 \\
\hline 40,00 & 0,6001 & 0,5989 & 0,0012 \\
\hline 45,00 & 0,6424 & 0,6413 & 0,0011 \\
\hline
\end{tabular}

\begin{tabular}{|r|r|r|}
\hline \multicolumn{2}{|c|}{$\begin{array}{c}\sigma 2 \mathrm{eex}(\mathrm{z}) \\
{[\text { ton/cm }}\end{array}$} & \\
\cline { 1 - 2 } Teoria & $\begin{array}{c}\text { Elementos } \\
\text { Finitos }\end{array}$ & Diferença \\
\hline$-0,3860$ & $-0,3785$ & $\mathbf{0 , 0 0 7 5}$ \\
\hline$-0,1677$ & $-0,1635$ & 0,0042 \\
\hline 0,0539 & 0,0555 & 0,0017 \\
\hline 0,2537 & 0,2537 & 0,0000 \\
\hline 0,4191 & 0,4183 & 0,0008 \\
\hline 0,5464 & 0,5452 & 0,0012 \\
\hline 0,6373 & 0,6361 & 0,0012 \\
\hline 0,6967 & 0,6957 & 0,0010 \\
\hline 0,7308 & 0,7300 & 0,0008 \\
\hline 0,7461 & 0,7454 & 0,0007 \\
\hline
\end{tabular}




\begin{tabular}{|c|r|r|r|}
\cline { 2 - 3 } \multicolumn{1}{c|}{} & \multicolumn{2}{c|}{$\left.\begin{array}{c}\multicolumn{1}{c}{} \\
{[\text { ton }}\end{array} \mathrm{cm}^{2}\right]$} & \multicolumn{1}{c}{} \\
\hline $\begin{array}{c}z \\
{[\mathrm{~cm}]}\end{array}$ & Teoria & $\begin{array}{c}\text { Elementos } \\
\text { Finitos }\end{array}$ & Diferença \\
\hline 50,00 & 0,6715 & 0,6706 & 0,0010 \\
\hline 55,00 & 0,6895 & 0,6886 & 0,0009 \\
\hline 60,00 & 0,6987 & 0,6977 & 0,0010 \\
\hline 65,00 & 0,7013 & 0,7002 & 0,0011 \\
\hline 70,00 & 0,6995 & 0,6983 & 0,0012 \\
\hline 75,00 & 0,6949 & 0,6936 & 0,0014 \\
\hline 80,00 & 0,6889 & 0,6874 & 0,0015 \\
\hline 85,00 & 0,6823 & 0,6806 & 0,0017 \\
\hline 90,00 & 0,6758 & 0,6740 & 0,0018 \\
\hline 95,00 & 0,6696 & 0,6677 & 0,0019 \\
\hline 100,00 & 0,6641 & 0,6621 & 0,0020 \\
\hline 105,00 & 0,6591 & 0,6571 & 0,0020 \\
\hline 110,00 & 0,6548 & 0,6527 & 0,0021 \\
\hline 115,00 & 0,6509 & 0,6488 & 0,0021 \\
\hline 120,00 & 0,6474 & 0,6453 & 0,0021 \\
\hline 125,00 & 0,6441 & 0,6421 & 0,0021 \\
\hline
\end{tabular}

\begin{tabular}{|c|c|c|}
\hline \multicolumn{2}{|c|}{$\begin{array}{l}\sigma 2 \operatorname{eex}(\mathrm{z}) \\
{\left[\text { ton } / \mathrm{cm}^{2}\right]}\end{array}$} & \\
\hline Teoria & $\begin{array}{c}\text { Elementos } \\
\text { Finitos }\end{array}$ & Diferença \\
\hline 0,7483 & 0,7476 & 0,0007 \\
\hline 0,7422 & 0,7414 & 0,0008 \\
\hline 0,7316 & 0,7306 & 0,0010 \\
\hline 0,7190 & 0,7178 & 0,0012 \\
\hline 0,7063 & 0,7049 & 0,0014 \\
\hline 0,6945 & 0,6929 & 0,0016 \\
\hline 0,6843 & 0,6825 & 0,0018 \\
\hline 0,6757 & 0,6738 & 0,0020 \\
\hline 0,6687 & 0,6667 & 0,0021 \\
\hline 0,6630 & 0,6609 & 0,0021 \\
\hline 0,6585 & 0,6563 & 0,0022 \\
\hline 0,6547 & 0,6525 & 0,0022 \\
\hline 0,6515 & 0,6493 & 0,0022 \\
\hline 0,6486 & 0,6465 & 0,0021 \\
\hline 0,6460 & 0,6438 & 0,0021 \\
\hline 0,6434 & 0,6413 & 0,0021 \\
\hline
\end{tabular}
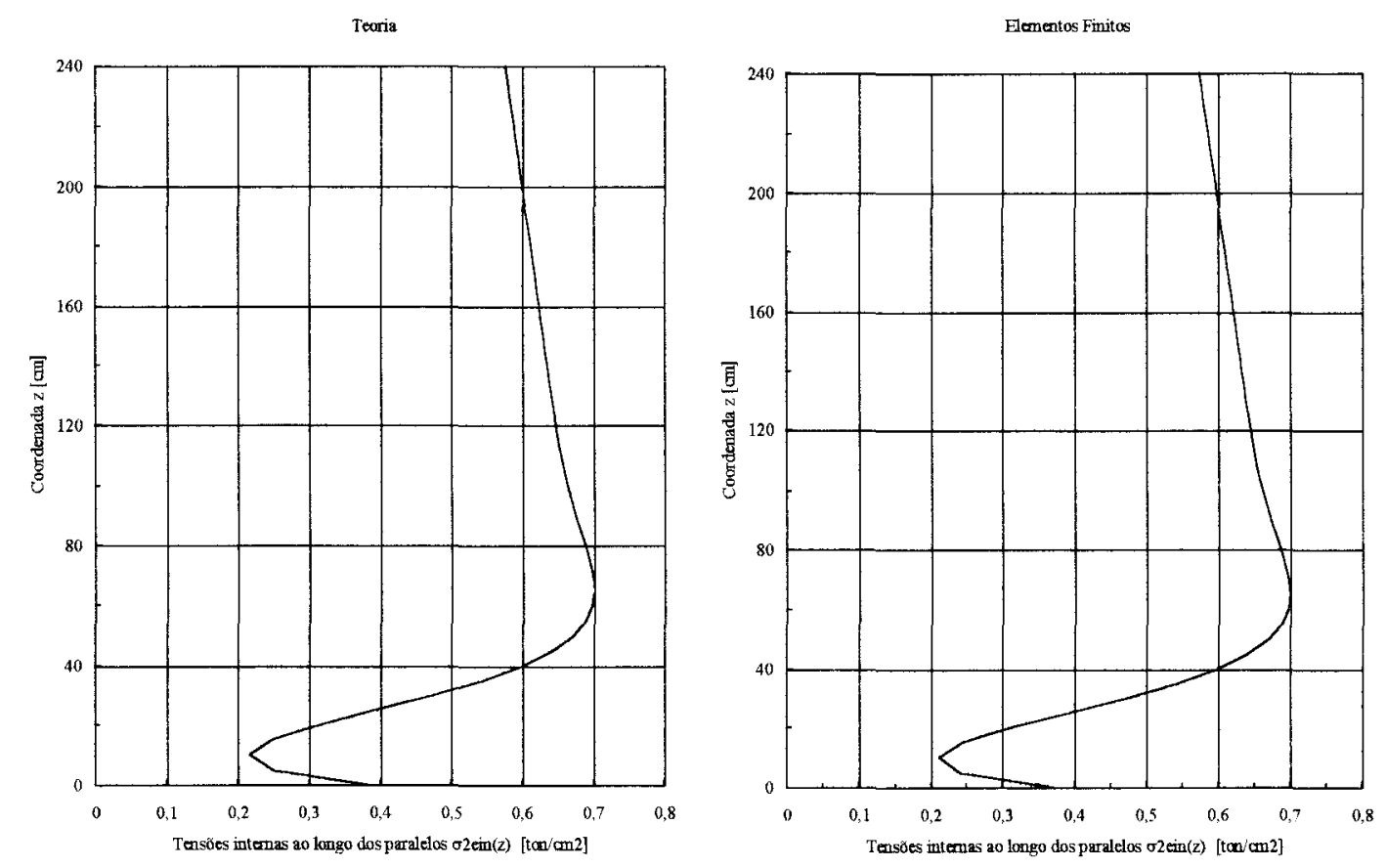

FIGURA 37 - Gráficos para os valores das tensões ao longo dos paralelos da superficie interna $\sigma 2 \operatorname{ein}(\mathrm{z})$ da casca cilíndrica 

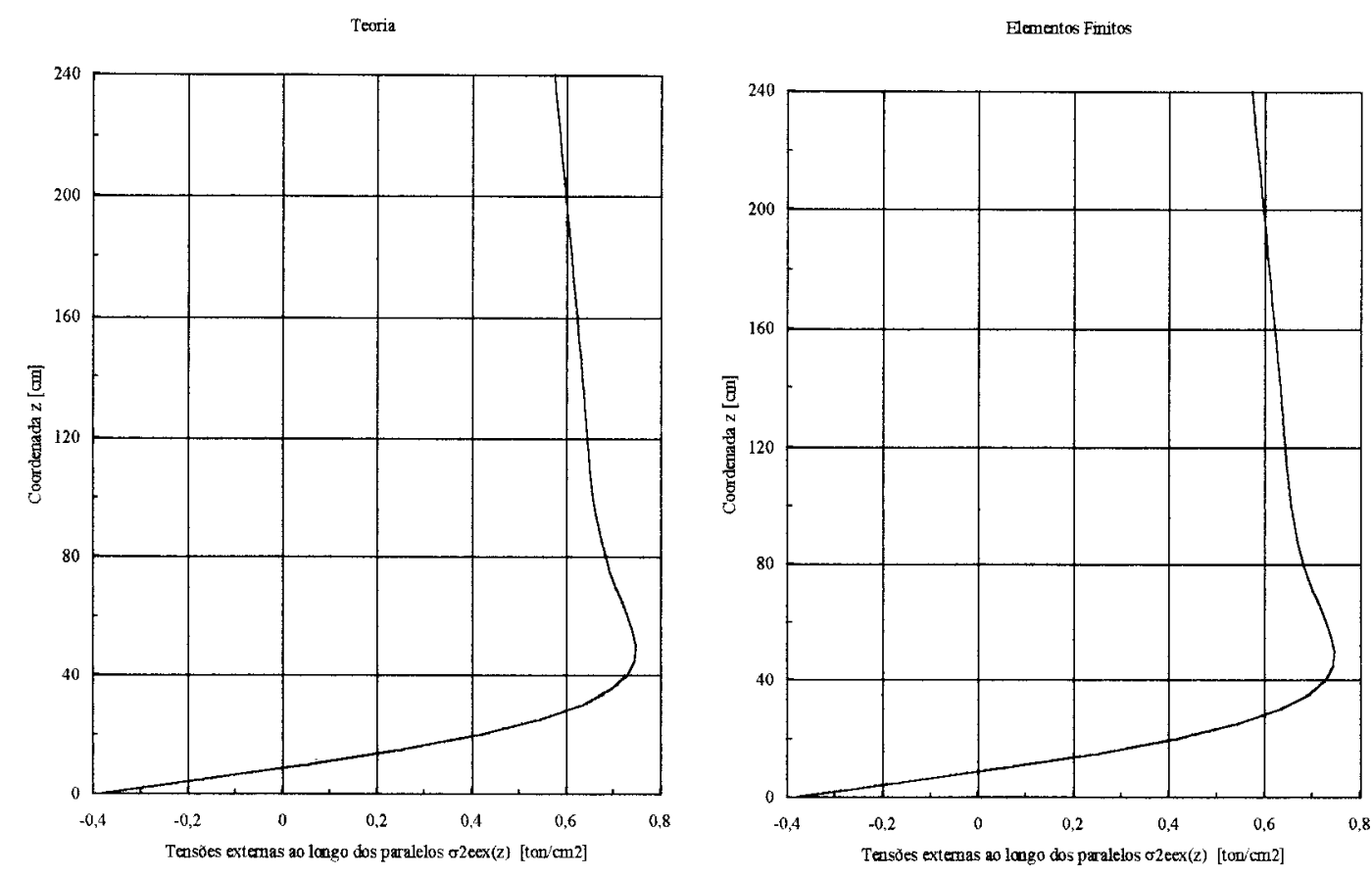

FIGURA 38 - Gráficos para os valores das tensões ao longo dos paralelos da superfície externa $\sigma 2 \operatorname{eex}(\mathrm{z})$ da casca cilíndrica

Apenas para comparação são apresentados abaixo os valores encontrados para as tensões de membrana atuantes ao longo dos meridianos e dos paralelos da superficie média da casca cilíndrica :

TABELA 5 - Valores para as tensões teóricas de membrana ao longo dos meridianos $\sigma \operatorname{lm}(\mathrm{z})$ e paralelos $\sigma 2 \mathrm{~m}(\mathrm{z})$ da superficie média da casca

\begin{tabular}{|c|r|r|}
\hline $\begin{array}{c}\mathrm{z} \\
{[\mathrm{cm}]}\end{array}$ & $\begin{array}{c}\sigma 1 \mathrm{~m}(\mathrm{z}) \\
{\left[\mathrm{ton} / \mathrm{cm}^{2}\right]}\end{array}$ & $\begin{array}{c}\sigma 2 \mathrm{~m}(\mathrm{z}) \\
{\left[\mathrm{ton} / \mathrm{cm}^{2}\right]}\end{array}$ \\
\hline 0,00 & 0,0000 & 0,7200 \\
\hline 5,00 & 0,0000 & 0,7170 \\
\hline 10,00 & 0,0000 & 0,7140 \\
\hline 15,00 & 0,0000 & 0,7110 \\
\hline 20,00 & 0,0000 & 0,7080 \\
\hline 25,00 & 0,0000 & 0,7050 \\
\hline 30,00 & 0,0000 & 0,7020 \\
\hline 35,00 & 0,0000 & 0,6990 \\
\hline 40,00 & 0,0000 & 0,6960 \\
\hline 45,00 & 0,0000 & 0,6930 \\
\hline
\end{tabular}

\begin{tabular}{|c|r|r|}
\hline $\begin{array}{c}\mathrm{z} \\
{[\mathrm{cm}]}\end{array}$ & $\begin{array}{c}\sigma 1 \mathrm{~m}(\mathrm{z}) \\
{\left[\text { ton } / \mathrm{cm}^{2}\right]}\end{array}$ & $\begin{array}{c}\sigma 2 \mathrm{~m}(\mathrm{z}) \\
{\left[\text { ton } / \mathrm{cm}^{2}\right]}\end{array}$ \\
\hline 65,00 & 0,0000 & 0,6810 \\
\hline 70,00 & 0,0000 & 0,6780 \\
\hline 75,00 & 0,0000 & 0,6750 \\
\hline 80,00 & 0,0000 & 0,6720 \\
\hline 85,00 & 0,0000 & 0,6690 \\
\hline 90,00 & 0,0000 & 0,6660 \\
\hline 95,00 & 0,0000 & 0,6630 \\
\hline 100,00 & 0,0000 & 0,6600 \\
\hline 105,00 & 0,0000 & 0,6570 \\
\hline 110,00 & 0,0000 & 0,6540 \\
\hline
\end{tabular}




\begin{tabular}{|c|r|r|}
\hline $\begin{array}{c}\mathrm{z} \\
{[\mathrm{cm}]}\end{array}$ & $\begin{array}{c}\sigma 1 \mathrm{~m}(\mathrm{z}) \\
{\left[\text { ton } / \mathrm{cm}^{2}\right]}\end{array}$ & $\begin{array}{c}\sigma 2 \mathrm{~m}(\mathrm{z}) \\
{\left[\text { ton } / \mathrm{cm}^{2}\right]}\end{array}$ \\
\hline 50,00 & 0,0000 & 0,6900 \\
\hline 55,00 & 0,0000 & 0,6870 \\
\hline 60,00 & 0,0000 & 0,6840 \\
\hline
\end{tabular}

\begin{tabular}{|c|r|r|}
\hline $\begin{array}{c}\mathrm{z} \\
{[\mathrm{cm}]}\end{array}$ & $\begin{array}{c}\sigma 1 \mathrm{~m}(\mathrm{z}) \\
{\left[\text { ton } / \mathrm{cm}^{2}\right]}\end{array}$ & $\begin{array}{c}\sigma 2 \mathrm{~m}(\mathrm{z}) \\
{\left[\text { ton } / \mathrm{cm}^{2}\right]}\end{array}$ \\
\hline 115,00 & 0,0000 & 0,6510 \\
\hline 120,00 & 0,0000 & 0,6480 \\
\hline 125,00 & 0,0000 & 0,6450 \\
\hline
\end{tabular}

Pode-se ver abaixo no gráfico com as tensões teóricas ao longo dos paralelos da casca cilíndrica, que as mesmas possuem seus valores praticamente coincidêntes acima da região de $\mathrm{z}=\lambda=119,73[\mathrm{~cm}]$.

Teoria

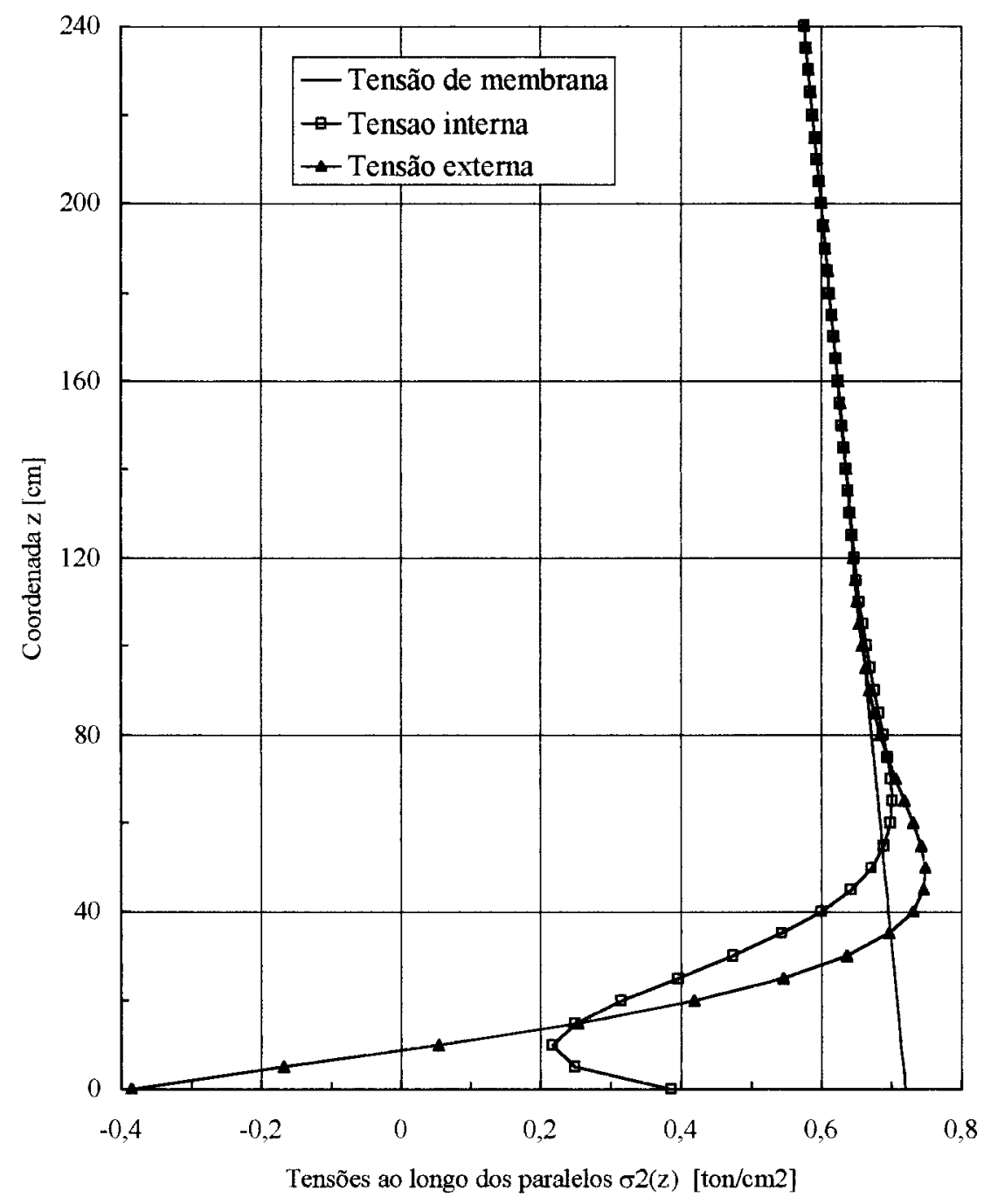

FIGURA 39 - Gráfico comparativo para os valores das tensões ao longo dos paralelos da casca cilíndrica : $\sigma 2 \mathrm{~m}(\mathrm{z}), \sigma 2 \operatorname{ein}(\mathrm{z})$ e $\sigma 2 \operatorname{eex}(\mathrm{z})$ 


\subsubsection{CASCA CILÍNDRICA COM BORDA INFERIOR APOIADA} FIXA

Para os deslocamentos radiais e rotações ao longo do meridiano da superfície média da casca cilíndrica encontrou-se :

TABELA 6 - Valores dos deslocamentos radiais $\mathrm{Wa}(\mathrm{z})$ e rotações $\phi \mathrm{a}(\mathrm{z})$

\begin{tabular}{|c|r|r|r|}
\cline { 2 - 3 } \multicolumn{1}{c|}{} & \multicolumn{2}{c|}{ Wa(z) } & \multicolumn{1}{c}{$[\mathrm{cm}]$} \\
\hline $\begin{array}{c}\mathrm{z} \\
{[\mathrm{cm}]}\end{array}$ & Teoria & $\begin{array}{c}\text { Elementos } \\
\text { Finitos }\end{array}$ & Diferença \\
\hline 0,00 & 0,0000 & 0,0000 & 0,0000 \\
\hline 5,00 & 0,0520 & 0,0515 & 0,0005 \\
\hline 10,00 & 0,0987 & 0,0979 & 0,0008 \\
\hline 15,00 & 0,1371 & 0,1362 & $\mathbf{0 , 0 0 0 9}$ \\
\hline 20,00 & 0,1664 & 0,1656 & 0,0008 \\
\hline 25,00 & 0,1872 & 0,1866 & 0,0006 \\
\hline 30,00 & 0,2007 & 0,2002 & 0,0005 \\
\hline 35,00 & 0,2083 & 0,2079 & 0,0004 \\
\hline 40,00 & 0,2116 & 0,2112 & 0,0004 \\
\hline 45,00 & 0,2118 & 0,2115 & 0,0003 \\
\hline 50,00 & 0,2101 & 0,2098 & 0,0003 \\
\hline 55,00 & 0,2074 & 0,2071 & 0,0003 \\
\hline 60,00 & 0,2043 & 0,2039 & 0,0004 \\
\hline 65,00 & 0,2011 & 0,2007 & 0,0004 \\
\hline 70,00 & 0,1982 & 0,1978 & 0,0004 \\
\hline 75,00 & 0,1957 & 0,1952 & 0,0005 \\
\hline 80,00 & 0,1935 & 0,1930 & 0,0005 \\
\hline 85,00 & 0,1917 & 0,1912 & 0,0005 \\
\hline 90,00 & 0,1903 & 0,1897 & 0,0006 \\
\hline 95,00 & 0,1890 & 0,1884 & 0,0006 \\
\hline 100,00 & 0,1880 & 0,1874 & 0,0006 \\
\hline 105,00 & 0,1871 & 0,1865 & 0,0006 \\
\hline 110,00 & 0,1863 & 0,1857 & 0,0006 \\
\hline 115,00 & 0,1855 & 0,1849 & 0,0006 \\
\hline 120,00 & 0,1848 & 0,1842 & 0,0006 \\
\hline 125,00 & 0,1840 & 0,1834 & 0,0006 \\
\hline & & & \\
\hline
\end{tabular}

\begin{tabular}{|c|c|c|}
\hline \multicolumn{2}{|c|}{$\begin{array}{r} \\
\text { [radianos] }\end{array}$} & \multicolumn{1}{c}{} \\
\cline { 1 - 2 } Teoria & $\begin{array}{c}\text { Elementos } \\
\text { Finitos }\end{array}$ & Diferença \\
\hline 0,0106 & 0,0105 & $\mathbf{0 , 0 0 0 1}$ \\
\hline 0,0100 & 0,0099 & 0,0001 \\
\hline 0,0086 & 0,0085 & 0,0000 \\
\hline 0,0068 & 0,0068 & 0,0000 \\
\hline 0,0050 & 0,0050 & 0,0000 \\
\hline 0,0034 & 0,0034 & 0,0000 \\
\hline 0,0021 & 0,0021 & 0,0000 \\
\hline 0,0010 & 0,0011 & 0,0000 \\
\hline 0,0003 & 0,0003 & 0,0000 \\
\hline$-0,0002$ & $-0,0002$ & 0,0000 \\
\hline$-0,0005$ & $-0,0005$ & 0,0000 \\
\hline$-0,0006$ & $-0,0006$ & 0,0000 \\
\hline$-0,0006$ & $-0,0006$ & 0,0000 \\
\hline$-0,0006$ & $-0,0006$ & 0,0000 \\
\hline$-0,0005$ & $-0,0006$ & 0,0000 \\
\hline$-0,0005$ & $-0,0005$ & 0,0000 \\
\hline$-0,0004$ & $-0,0004$ & 0,0000 \\
\hline$-0,0003$ & $-0,0003$ & 0,0000 \\
\hline$-0,0003$ & $-0,0003$ & 0,0000 \\
\hline$-0,0002$ & $-0,0002$ & 0,0000 \\
\hline$-0,0002$ & $-0,0002$ & 0,0000 \\
\hline$-0,0002$ & $-0,0002$ & 0,0000 \\
\hline$-0,0002$ & $-0,0002$ & 0,0000 \\
\hline$-0,0002$ & $-0,0002$ & 0,0000 \\
\hline$-0,0002$ & $-0,0002$ & 0,0000 \\
\hline$-0,0002$ & $-0,0002$ & 0,0000 \\
\hline & & \\
\cline { 1 - 1 } & &
\end{tabular}



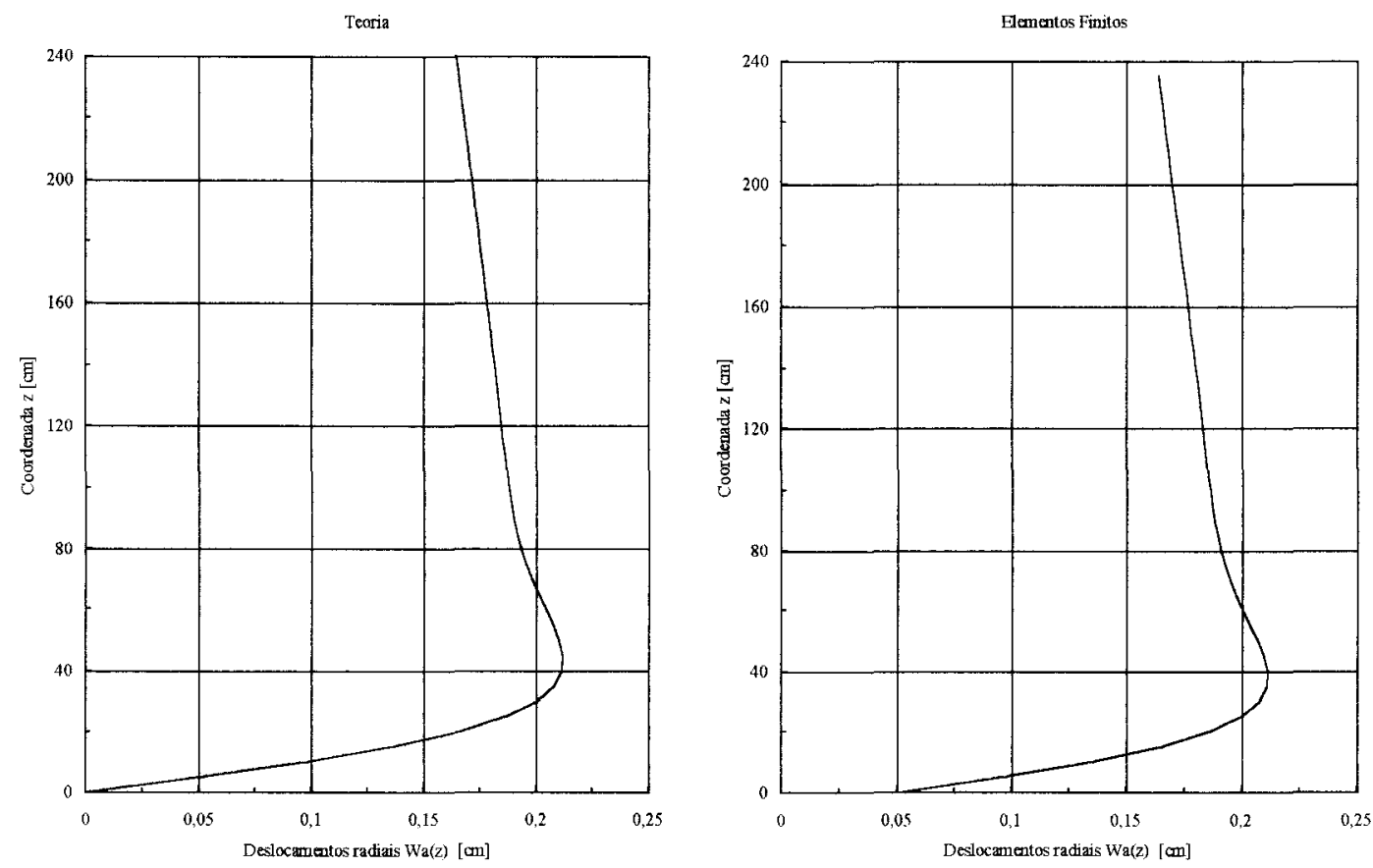

FIGURA 40 - Gráficos para os valores dos deslocamentos radiais Wa(z) ao longo do meridiano da superficie média da casca cilíndrica
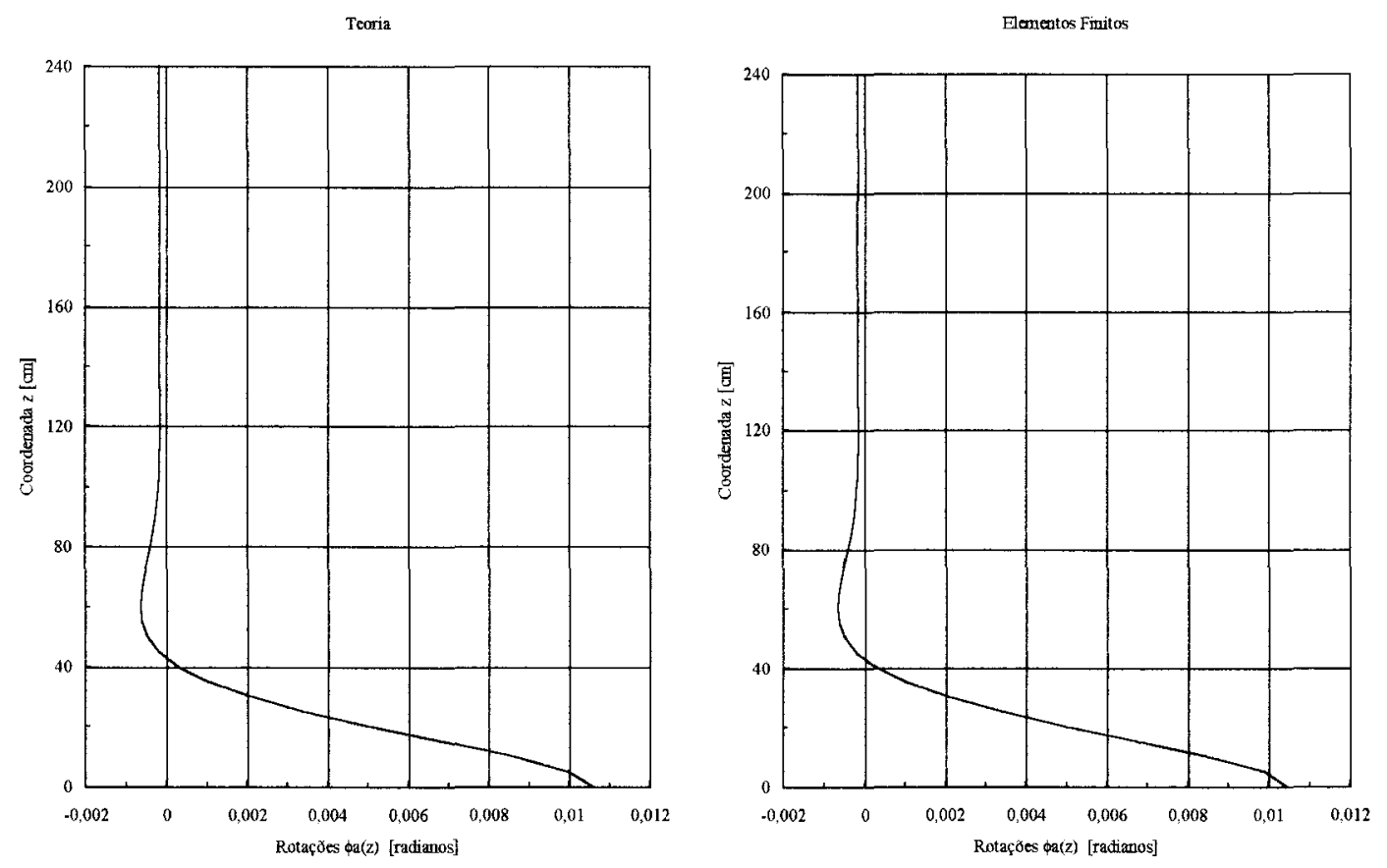

FIGURA 41 - Gráficos para os valores das rotações $\phi \mathrm{a}(\mathrm{z})$ ao longo do meridiano da superficie média da casca cilíndrica 
Os momentos solicitantes ao longo dos paralelos e dos meridianos da superficie média da casca cilíndrica e suas correspondentes diferenças absolutas foram

TABELA 7 - Valores dos momentos ao longo dos paralelos $\mathrm{M} 1 \mathrm{a}(\mathrm{z})$ e dos meridianos M2a(z)

\begin{tabular}{|c|c|c|c|c|c|c|}
\hline \multirow[b]{2}{*}{$\begin{array}{c}\mathrm{z} \\
{[\mathrm{cm}]}\end{array}$} & \multicolumn{2}{|c|}{$\begin{array}{c}\mathrm{Mla}(\mathrm{z}) \\
{[\text { ton } \mathrm{cm} / \mathrm{cm}]}\end{array}$} & \multirow[b]{2}{*}{ Diferença } & \multicolumn{2}{|c|}{$\begin{array}{c}\mathrm{M} 2 \mathrm{a}(\mathrm{z}) \\
{[\text { ton } \mathrm{cm} / \mathrm{cm} \text { ] }}\end{array}$} & \multirow[b]{2}{*}{ Diferença } \\
\hline & Teoria & $\begin{array}{c}\text { Elementos } \\
\text { Finitos }\end{array}$ & & Teoria & $\begin{array}{l}\text { Elementos } \\
\text { Finitos }\end{array}$ & \\
\hline 0,00 & 0,0000 & $-0,0001$ & 0,0001 & 0,0000 & 0,0000 & 0,0000 \\
\hline 5,00 & 0,0435 & 0,0430 & 0,0004 & 0,0130 & 0,0129 & 0,0001 \\
\hline 10,00 & 0,0646 & 0,0641 & 0,0005 & 0,0194 & 0,0192 & (0,0002 \\
\hline 15,00 & 0,0702 & 0,0699 & 0,0004 & 0,0211 & 0,0210 & 0,0001 \\
\hline 20,00 & 0,0662 & 0,0659 & 0,0002 & 0,0198 & 0,0198 & 0,0001 \\
\hline 25,00 & 0,0567 & 0,0567 & 0,0001 & 0,0170 & 0,0170 & 0,0000 \\
\hline 30,00 & 0,0451 & 0,0452 & 0,0000 & 0,0135 & 0,0136 & 0,0000 \\
\hline 35,00 & 0,0335 & 0,0336 & 0,0001 & 0,0100 & 0,0101 & 0,0000 \\
\hline 40,00 & 0,0231 & 0,0232 & 0,0001 & 0,0069 & 0,0070 & 0,0000 \\
\hline 45,00 & 0,0144 & 0,0145 & 0,0001 & 0,0043 & 0,0044 & 0,0000 \\
\hline 50,00 & 0,0078 & 0,0079 & 0,0000 & 0,0023 & 0,0024 & 0,0000 \\
\hline 55,00 & 0,0031 & 0,0031 & 0,0000 & 0,0009 & 0,0009 & 0,0000 \\
\hline 60,00 & $-0,0001$ & $-0,0001$ & 0,0000 & 0,0000 & 0,0000 & 0,0000 \\
\hline 65,00 & $-0,0019$ & $-0,0020$ & & $-0,0006$ & $-0,0006$ & 0,0000 \\
\hline 70,00 & $-0,0028$ & $-0,0029$ & 0,0001 & $-0,0008$ & $-0,0009$ & 0,0000 \\
\hline 75,00 & $-0,0030$ & $-0,0031$ & 0,0001 & $-0,0009$ & $-0,0009$ & 0,0000 \\
\hline 80,00 & $-0,0029$ & $-0,0029$ & 0,0001 & $-0,0009$ & $-0,0009$ & 0,0000 \\
\hline 85,00 & $-0,0024$ & $-0,0025$ & 0,0001 & $-0,0007$ & $-0,0008$ & 0,0000 \\
\hline 90,00 & $-0,0019$ & $-0,0020$ & 0,0001 & $-0,0006$ & $-0,0006$ & 0,0000 \\
\hline 95,00 & $-0,0014$ & $-0,0015$ & 0,0000 & $-0,0004$ & $-0,0004$ & 0,0000 \\
\hline 100,00 & $-0,0010$ & $-0,0010$ & 0,0000 & $-0,0003$ & $-0,0003$ & 0,0000 \\
\hline 105,00 & $-0,0006$ & $-0,0006$ & 0,0000 & $-0,0002$ & $-0,0002$ & 0,0000 \\
\hline 110,00 & $-0,0003$ & $-0,0003$ & 0,0000 & $-0,0001$ & $-0,0001$ & 0,0000 \\
\hline 115,00 & $-0,0001$ & $-0,0001$ & 0,0000 & 0,0000 & 0,0000 & 0,0000 \\
\hline 120,00 & 0,0000 & 0,0000 & 0,0000 & 0,0000 & 0,0000 & 0,0000 \\
\hline 125,00 & 0,0001 & 0,0001 & 0,0000 & 0,0000 & 0,0000 & 0,0000 \\
\hline
\end{tabular}


Teoria

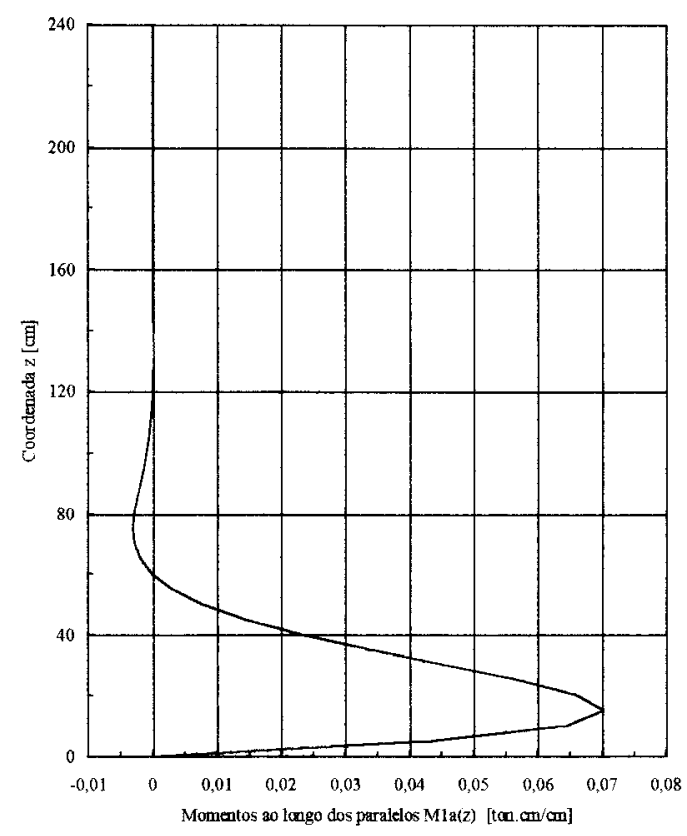

Elementos Finitos

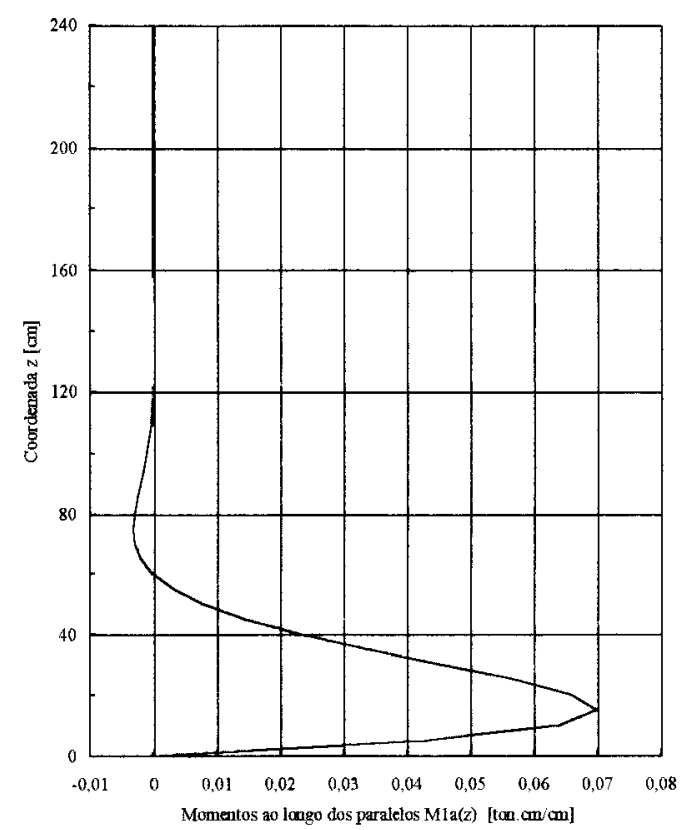

FIGURA 42 - Gráficos para os valores dos momentos ao longo dos paralelos M1a(z) da superfície média da casca cilíndrica

Tentia

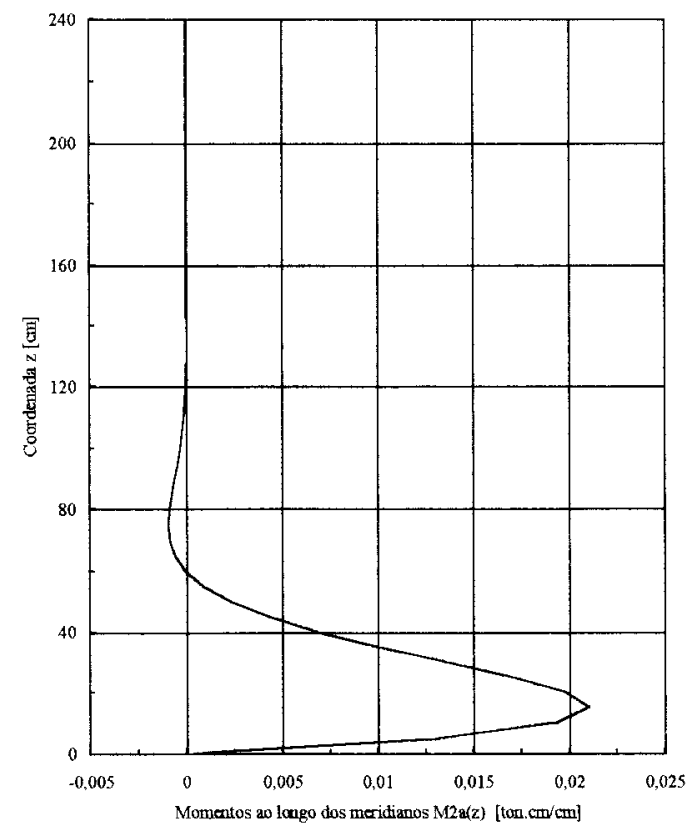

Elementos Fimitos

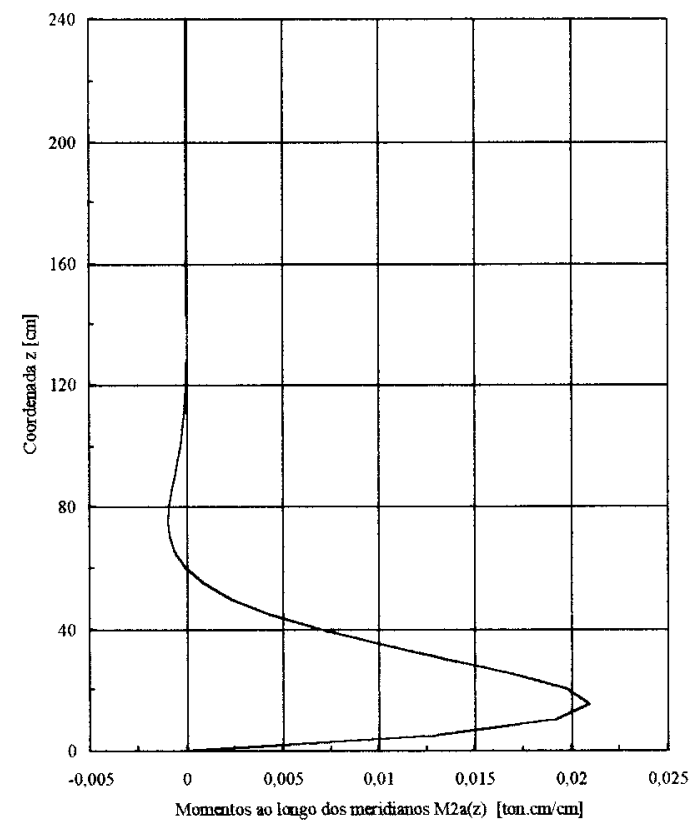

FIGURA 43 - Gráficos para os valores dos momentos ao longo dos meridianos M2a(z) da superficie média da casca cilíndrica 
Para as tensões atuantes ao longo dos meridianos da superfície interna e externa da casca cilíndrica obteve-se :

TABELA 8 - Valores para as tensões ao longo dos meridianos da superficie interna $\sigma$ lain(z) e externa $\sigma$ laex(z) da casca

\begin{tabular}{|c|c|c|c|}
\hline \multirow[b]{2}{*}{$\begin{array}{c}\mathrm{z} \\
{[\mathrm{cm}]}\end{array}$} & \multicolumn{2}{|c|}{$\begin{array}{l}\text { olain }(\mathrm{z}) \\
{\left[\text { ton } / \mathrm{cm}^{2}\right]}\end{array}$} & \multirow[b]{2}{*}{ Diferença } \\
\hline & Teoria & \begin{tabular}{|c|} 
Elementos \\
Finitos
\end{tabular} & \\
\hline 0,00 & 0,0000 & $-0,0289$ & 0,0289 \\
\hline 5,00 & $-0,2608$ & $-0,2568$ & 0,0040 \\
\hline 10,00 & $-0,3875$ & $-0,3823$ & 0,0053 \\
\hline 15,00 & $-0,4215$ & $-0,4166$ & 0,0048 \\
\hline 20,00 & $-0,3969$ & $-0,3932$ & 0,0037 \\
\hline 25,00 & $-0,3403$ & $-0,3379$ & 0,0024 \\
\hline 30,00 & $-0,2708$ & $-0,2694$ & 0,0014 \\
\hline 35,00 & $-0,2010$ & $-0,2003$ & 0,0007 \\
\hline 40,00 & $-0,1384$ & $-0,1381$ & 0,0003 \\
\hline 45,00 & $-0,0867$ & $-0,0866$ & 0,0001 \\
\hline 50,00 & $-0,0469$ & $-0,0468$ & 0,0001 \\
\hline 55,00 & $-0,0184$ & $-0,0182$ & 0,0002 \\
\hline 60,00 & 0,0004 & 0,0007 & 0,0003 \\
\hline 65,00 & 0,0115 & 0,0119 & 0,0004 \\
\hline 70,00 & 0,0168 & 0,0173 & 0,0004 \\
\hline 75,00 & 0,0182 & 0,0187 & 0,0004 \\
\hline 80,00 & 0,0171 & 0,0175 & 0,0004 \\
\hline 85,00 & 0,0146 & 0,0150 & 0,0004 \\
\hline 90,00 & 0,0116 & 0,0119 & 0,0003 \\
\hline 95,00 & 0,0086 & 0,0088 & 0,0002 \\
\hline 100,00 & 0,0059 & 0,0061 & 0,0002 \\
\hline 105,00 & 0,0037 & 0,0038 & 0,0001 \\
\hline 110,00 & 0,0020 & 0,0020 & 0,0000 \\
\hline 115,00 & 0,0008 & 0,0008 & 0,0000 \\
\hline 120,00 & 0,0000 & $-0,0001$ & 0,0000 \\
\hline 125,00 & $-0,0005$ & $-0,0005$ & 0,0000 \\
\hline
\end{tabular}

\begin{tabular}{|c|c|c|}
\hline \multicolumn{2}{|c|}{$\begin{array}{l}\text { olaex }(\mathrm{z}) \\
{\left[\text { ton } / \mathrm{cm}^{2}\right]}\end{array}$} & \\
\hline Teoria & \begin{tabular}{|l} 
Elementos \\
Finitos
\end{tabular} & Diferença \\
\hline 0,0000 & $-0,0305$ & 0,0305 \\
\hline 0,2608 & 0,2598 & 0,0011 \\
\hline 0,3875 & 0,3869 & 0,0006 \\
\hline 0,4215 & 0,4218 & 0,0003 \\
\hline 0,3969 & 0,3981 & 0,0012 \\
\hline 0,3403 & 0,3421 & 0,0018 \\
\hline 0,2708 & 0,2728 & 0,0020 \\
\hline 0,2010 & 0,2029 & 0,0019 \\
\hline 0,1384 & 0,1399 & 0,0015 \\
\hline 0,0867 & 0,0877 & 0,0010 \\
\hline 0,0469 & 0,0474 & 0,0005 \\
\hline 0,0184 & 0,0185 & 0,0001 \\
\hline$-0,0004$ & $-0,0007$ & 0,0003 \\
\hline$-0,0115$ & $-0,0120$ & 0,0005 \\
\hline$-0,0168$ & $-0,0175$ & 0,0006 \\
\hline$-0,0182$ & $-0,0189$ & 0,0007 \\
\hline$-0,0171$ & $-0,0177$ & 0,0006 \\
\hline$-0,0146$ & $-0,0152$ & 0,0006 \\
\hline$-0,0116$ & $-0,0121$ & 0,0005 \\
\hline$-0,0086$ & $-0,0090$ & 0,0003 \\
\hline$-0,0059$ & $-0,0062$ & 0,0002 \\
\hline$-0,0037$ & $-0,0038$ & 0,0001 \\
\hline$-0,0020$ & $-0,0021$ & 0,0001 \\
\hline$-0,0008$ & $-0,0008$ & 0,0000 \\
\hline 0,0000 & 0,0001 & 0,0000 \\
\hline 0,0005 & 0,0006 & 0,0000 \\
\hline
\end{tabular}



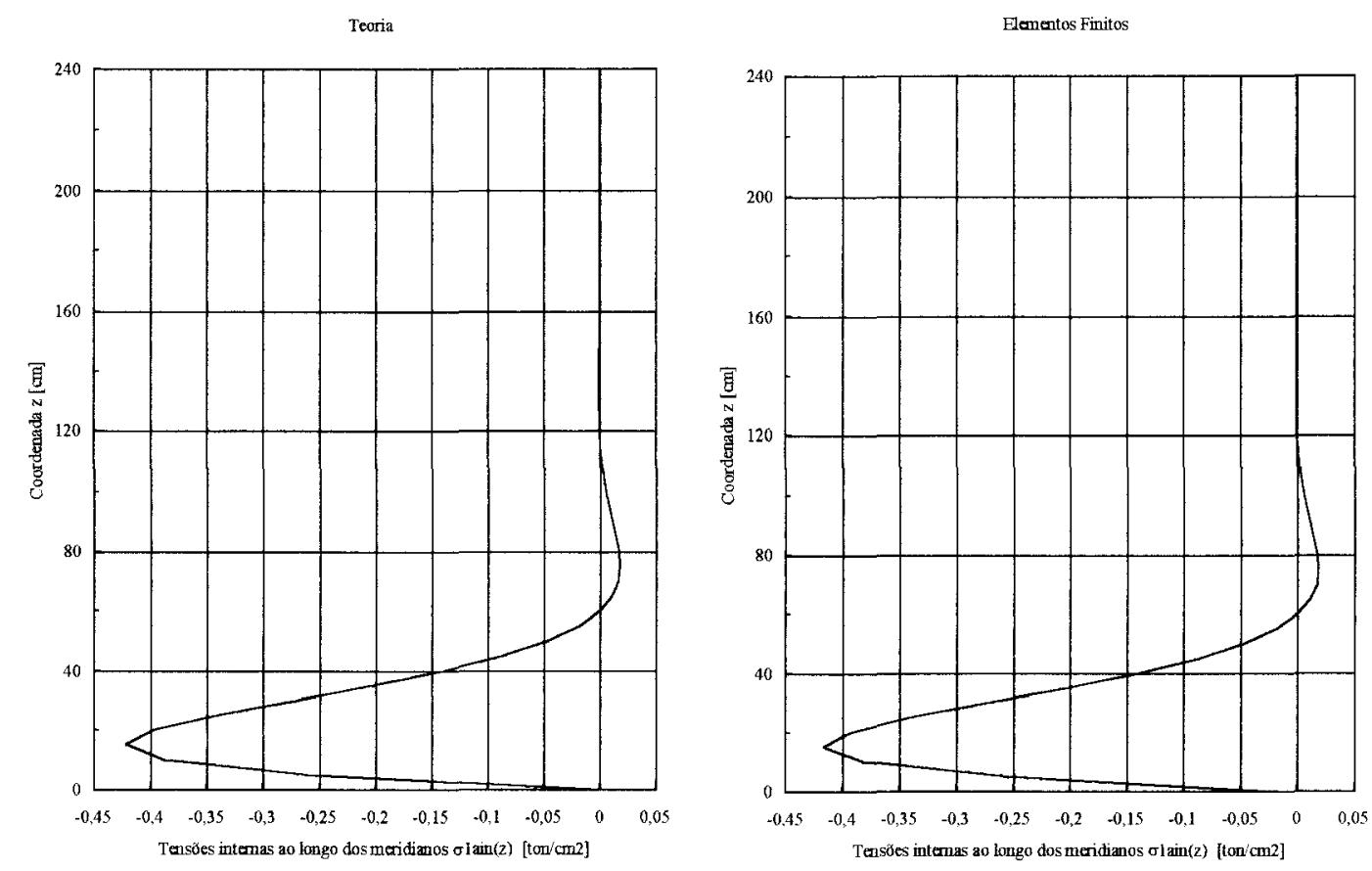

FIGURA 44 - Gráficos para os valores das tensões ao longo dos meridianos da superficie interna $\sigma$ lain(z) da casca cilíndrica
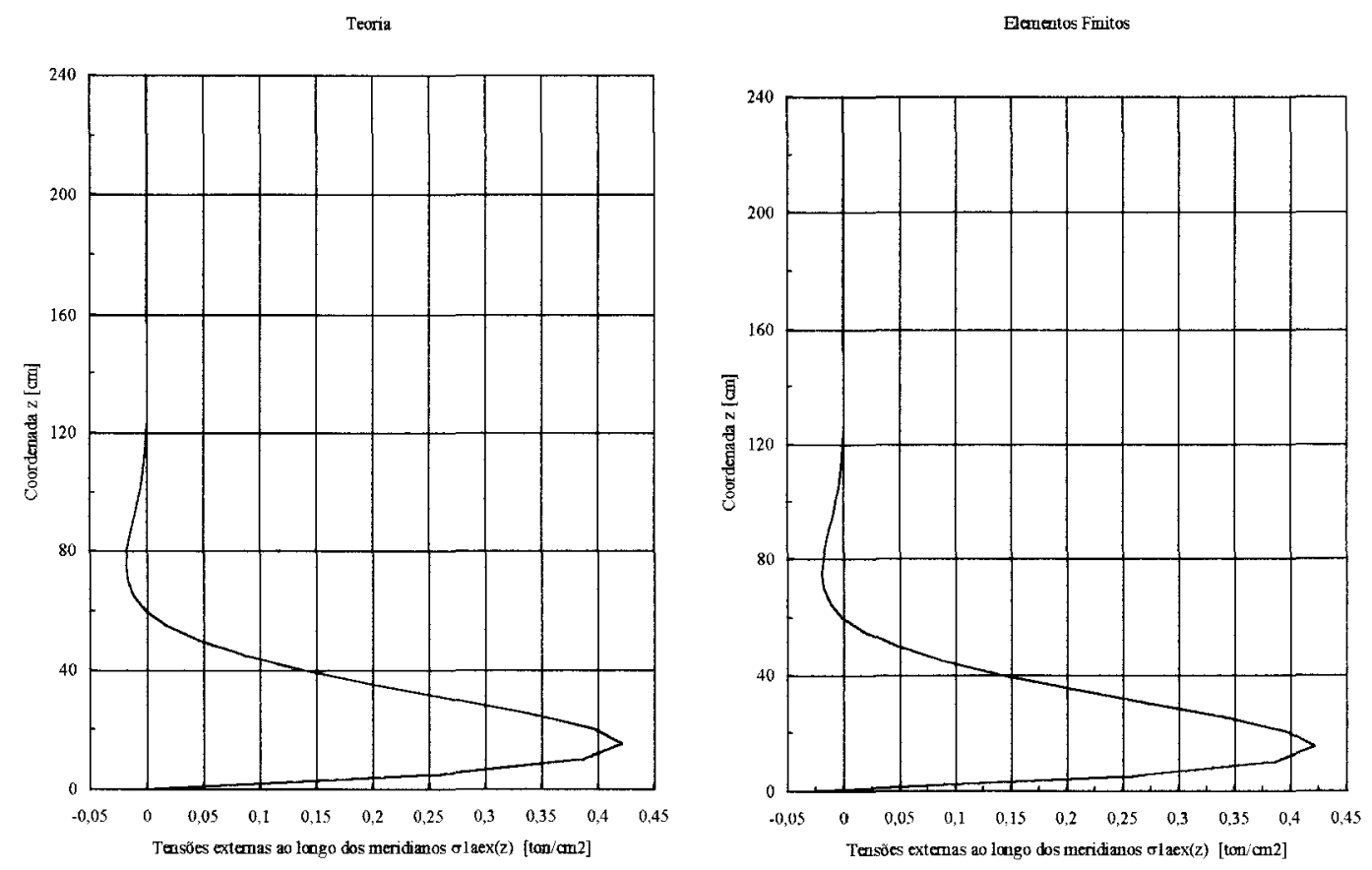

FIGURA 45 - Gráficos para os valores das tensões ao longo dos meridianos da superficie externa $\sigma 1 \mathrm{aex}(\mathrm{z})$ da casca cilíndrica 
E finalmente para as tensões atuantes ao longo dos paralelos da superficie interna e externa da casca cilíndrica encontrou-se :

TABELA 9 - Valores para as tensões ao longo dos paralelos da superfície interna $\sigma 2$ ain $(z)$ e externa $\sigma 2 \mathrm{aex}(\mathrm{z})$ da casca

\begin{tabular}{|c|c|c|c|}
\cline { 2 - 3 } \multicolumn{1}{c|}{} & \multicolumn{2}{c|}{\begin{tabular}{c}
\multicolumn{1}{c}{} \\
[ton
\end{tabular} $\mathrm{cm}^{2}$ ] } & \multicolumn{1}{c}{} \\
\hline $\begin{array}{c}z \\
{[\mathrm{~cm}]}\end{array}$ & Teoria & $\begin{array}{c}\text { Elementos } \\
\text { Finitos }\end{array}$ & Diferença \\
\hline 0,00 & 0,0000 & $-0,0087$ & $\mathbf{0 , 0 0 8 7}$ \\
\hline 5,00 & 0,1039 & 0,1032 & 0,0007 \\
\hline 10,00 & 0,2290 & 0,2278 & 0,0012 \\
\hline 15,00 & 0,3532 & 0,3517 & 0,0016 \\
\hline 20,00 & 0,4634 & 0,4618 & 0,0017 \\
\hline 25,00 & 0,5533 & 0,5517 & 0,0016 \\
\hline 30,00 & 0,6213 & 0,6199 & 0,0014 \\
\hline 35,00 & 0,6688 & 0,6677 & 0,0012 \\
\hline 40,00 & 0,6990 & 0,6979 & 0,0010 \\
\hline 45,00 & 0,7152 & 0,7143 & 0,0010 \\
\hline 50,00 & 0,7213 & 0,7203 & 0,0010 \\
\hline 55,00 & 0,7203 & 0,7193 & 0,0010 \\
\hline 60,00 & 0,7150 & 0,7139 & 0,0011 \\
\hline 65,00 & 0,7074 & 0,7060 & 0,0013 \\
\hline 70,00 & 0,6988 & 0,6973 & 0,0015 \\
\hline 75,00 & 0,6903 & 0,6887 & 0,0016 \\
\hline 80,00 & 0,6825 & 0,6807 & 0,0018 \\
\hline 85,00 & 0,6755 & 0,6735 & 0,0019 \\
\hline 90,00 & 0,6694 & 0,6674 & 0,0020 \\
\hline 95,00 & 0,6643 & 0,6622 & 0,0021 \\
\hline 100,00 & 0,6598 & 0,6577 & 0,0021 \\
\hline 105,00 & 0,6560 & 0,6539 & 0,0021 \\
\hline 110,00 & 0,6526 & 0,6505 & 0,0021 \\
\hline 115,00 & 0,6496 & 0,6475 & 0,0021 \\
\hline 120,00 & 0,6467 & 0,6446 & 0,0021 \\
\hline 125,00 & 0,6439 & 0,6418 & 0,0021 \\
\hline & & & \\
\hline
\end{tabular}

\begin{tabular}{|c|c|c|}
\hline \multicolumn{2}{|c|}{$\begin{array}{l}\sigma 2 \mathrm{aex}(\mathrm{z}) \\
{\left[\text { ton } / \mathrm{cm}^{2}\right]}\end{array}$} & \\
\hline Teoria & \begin{tabular}{|c|}
$\begin{array}{c}\text { Elementos } \\
\text { Finitos }\end{array}$ \\
\end{tabular} & Diferença \\
\hline 0,0000 & $-0,0092$ & 0,0092 \\
\hline 0,2604 & 0,2582 & 0,0022 \\
\hline 0,4616 & 0,4586 & 0,0030 \\
\hline 0,6061 & 0,6032 & 0,0029 \\
\hline 0,7015 & 0,6992 & 0,0024 \\
\hline 0,7575 & 0,7557 & 0,0018 \\
\hline 0,7838 & 0,7826 & 0,0012 \\
\hline 0,7894 & 0,7886 & 0,0008 \\
\hline 0,7820 & 0,7813 & 0,0007 \\
\hline 0,7673 & 0,7666 & 0,0007 \\
\hline 0,7495 & 0,7486 & 0,0008 \\
\hline 0,7314 & 0,7303 & 0,0011 \\
\hline 0,7148 & 0,7135 & 0,0013 \\
\hline 0,7005 & 0,6989 & 0,0016 \\
\hline 0,6887 & 0,6869 & 0,0018 \\
\hline 0,6794 & 0,6774 & 0,0020 \\
\hline 0,6722 & 0,6701 & 0,0021 \\
\hline 0,6667 & 0,6645 & 0,0022 \\
\hline 0,6624 & 0,6602 & 0,0022 \\
\hline 0,6591 & 0,6568 & 0,0022 \\
\hline 0,6563 & 0,6541 & 0,0022 \\
\hline 0,6538 & 0,6516 & 0,0022 \\
\hline 0,6514 & 0,6493 & 0,0022 \\
\hline 0,6491 & 0,6470 & 0,0021 \\
\hline 0,6467 & 0,6446 & 0,0021 \\
\hline 0,6442 & 0,6421 & 0,0020 \\
\hline
\end{tabular}



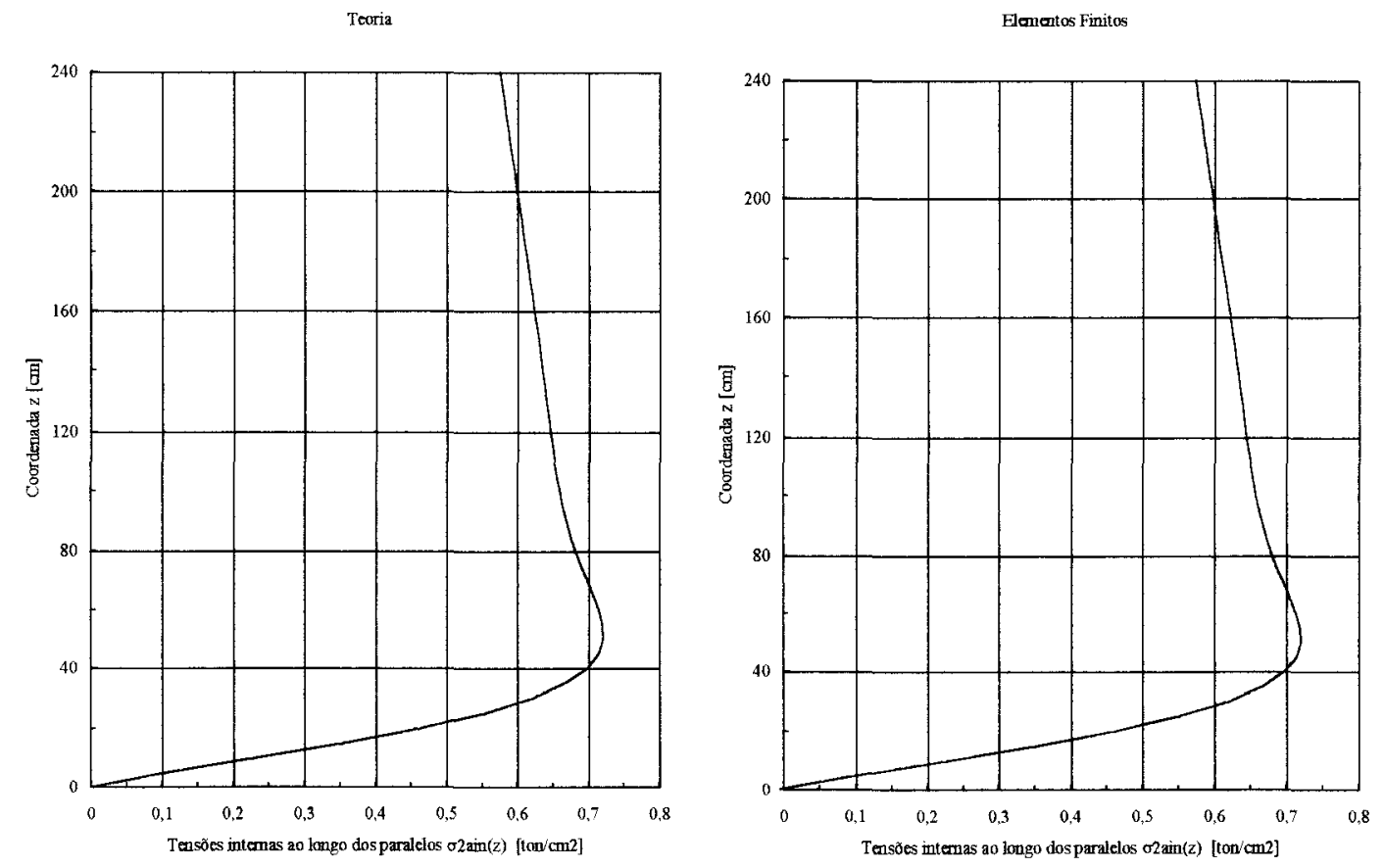

FIGURA 46 - Gráficos para os valores das tensões ao longo dos paralelos da superficie interna $\sigma 2$ ain(z) da casca cilíndrica
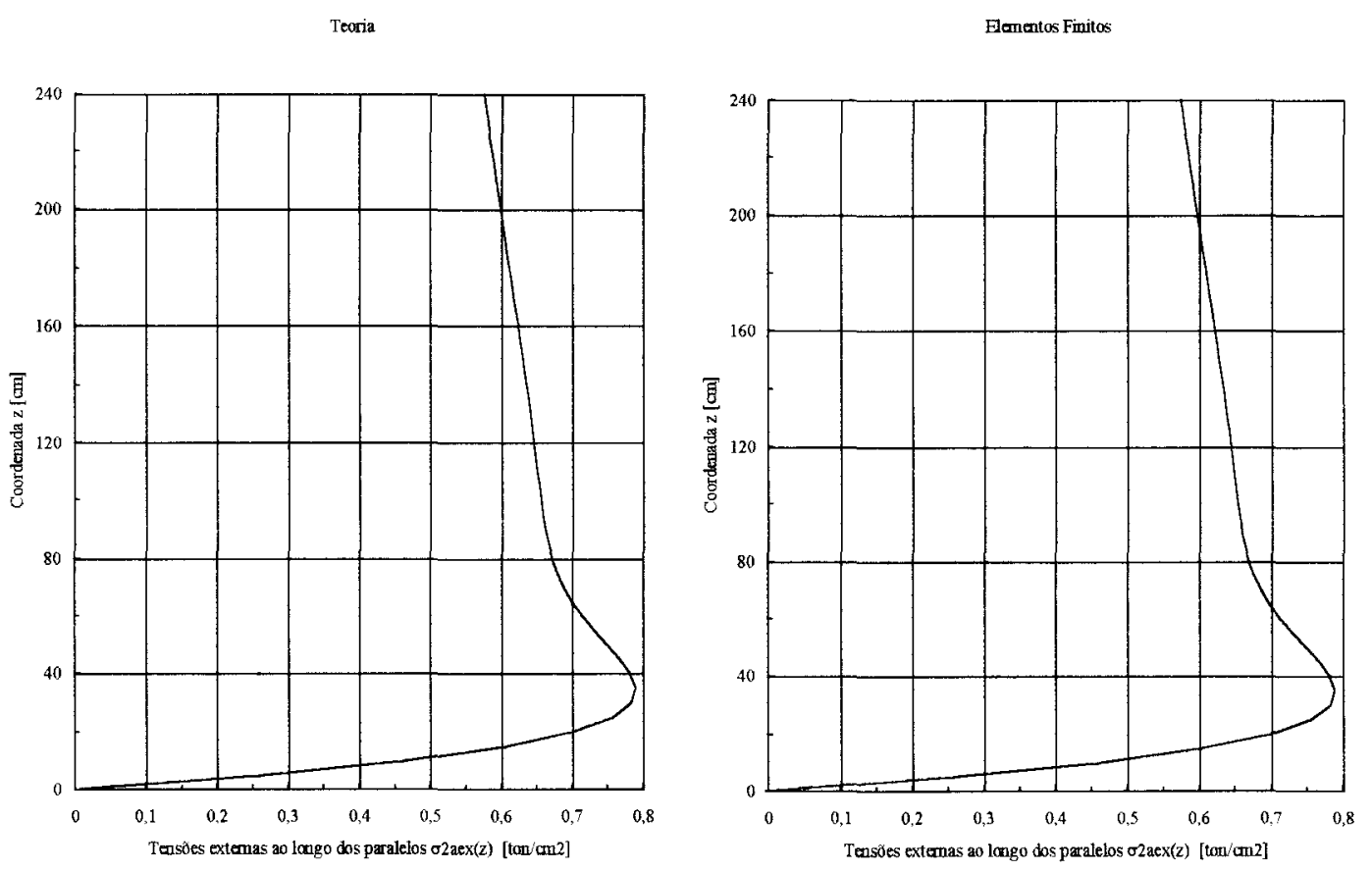

FIGURA 47 - Gráficos para os valores das tensões ao longo dos paralelos da superficie externa $\sigma 2 \mathrm{aex}(\mathrm{z})$ da casca cilíndrica 
E para comparação apresenta-se abaixo o gráfico com as tensões teóricas ao longo dos paralelos da casca cilíndrica que, como já visto para borda engastada, possuem seus valores praticamente coincidêntes acima da região de $\mathrm{Z}=\lambda=119,73$ $[\mathrm{cm}]$.

Teoria

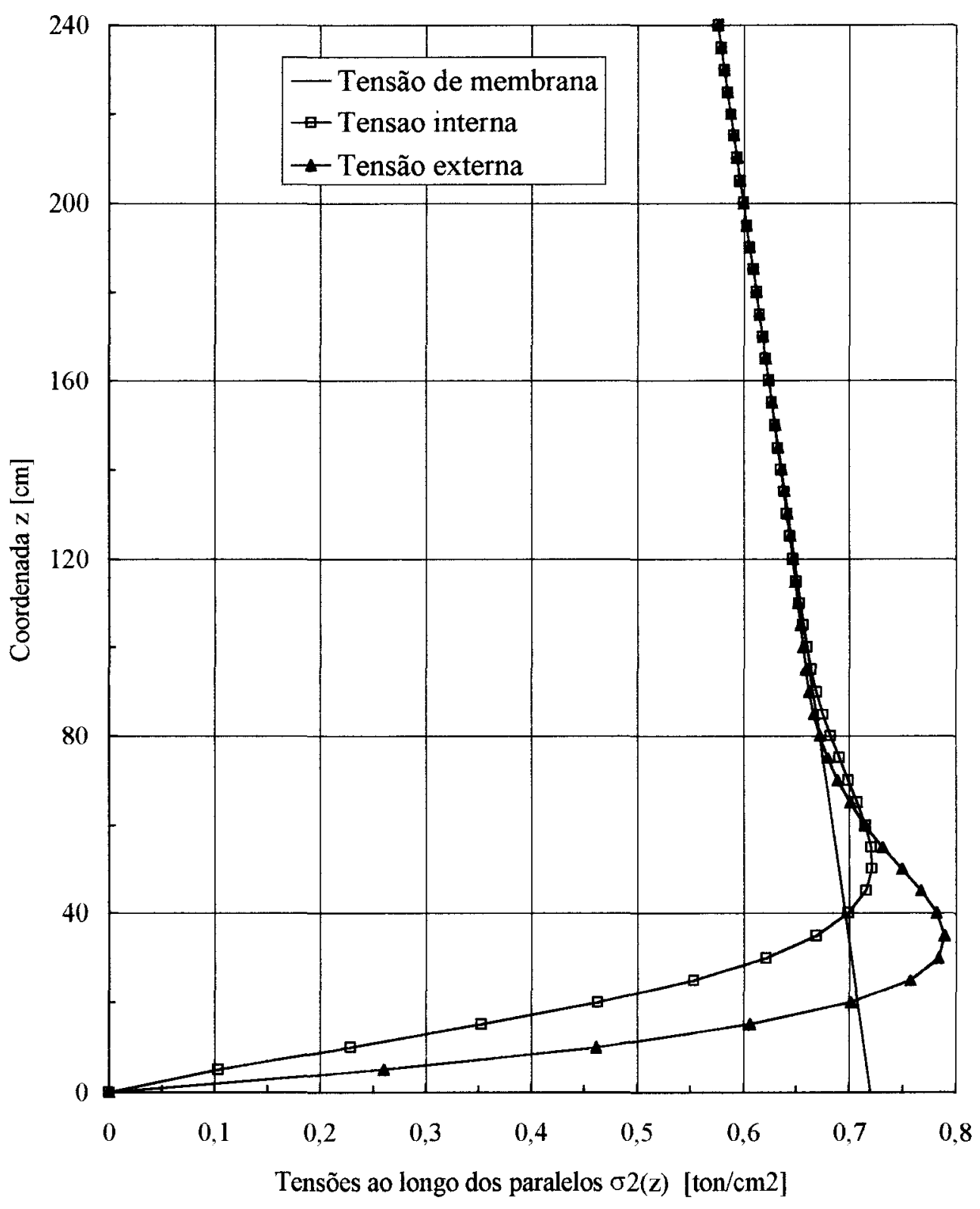

FIGURA 48 - Gráfico comparativo para os valores das tensões ao longo dos paralelos da casca cilíndrica : $\sigma 2 \mathrm{~m}(\mathrm{z}), \sigma 2 \mathrm{ain}(\mathrm{z})$ e $\sigma 2 \mathrm{aex}(\mathrm{z})$ 


\subsection{CALCULANDO E OBTENDO RESULTADOS PARA A CASCA CÔNICA INCLINAÇÃO $\theta \mathbf{r}=3^{\circ}$ COM A HORIZONTAL}

Através das equações teóricas do capítulo 2 para casca cônica, e com os dados abaixo :

\begin{tabular}{|c|c|c|c|c|c|c|}
\hline $\begin{array}{c}\mathrm{E} \\
{\left[\text { ton } / \mathrm{cm}^{2}\right]}\end{array}$ & $\begin{array}{c}v \\
(\text { Aço })\end{array}$ & $\begin{array}{c}\gamma \\
{\left[\text { ton } / \mathrm{cm}^{3}\right]}\end{array}$ & $\begin{array}{c}\theta \mathrm{r} \\
\text { [graus] }\end{array}$ & $\begin{array}{c}\alpha \mathrm{r} \\
\text { [graus] }\end{array}$ & $\begin{array}{c}\mathrm{D} \\
{[\mathrm{cm}]}\end{array}$ & $\begin{array}{c}\mathrm{r} \\
{[\mathrm{cm}]}\end{array}$ \\
\hline 2100 & 0,3 & $1,00 \mathrm{E}-06$ & 30 & 60 & 1200 & 600 \\
\hline
\end{tabular}

\begin{tabular}{|c|c|c|c|}
\hline $\begin{array}{c}\mathrm{R} \\
{[\mathrm{cm}]}\end{array}$ & $\begin{array}{c}\mathrm{H} \\
{[\mathrm{cm}]}\end{array}$ & $\begin{array}{c}\mathrm{h} \\
{[\mathrm{cm}]}\end{array}$ & $\begin{array}{c}\mathrm{t} \\
{[\mathrm{cm}]}\end{array}$ \\
\hline 1200 & 346,41 & 1200 & 1 \\
\hline
\end{tabular}

Encontram-se :

\begin{tabular}{|c|c|c|c|c|c|c|}
\hline $\begin{array}{c}\beta \\
{\left[\text { ton } / \mathrm{cm}^{3}\right]}\end{array}$ & $\begin{array}{c}\mathrm{B} \\
{[\text { ton } / \mathrm{cm}]}\end{array}$ & $\begin{array}{c}\alpha \\
{\left[\mathrm{cm}^{-1}\right]}\end{array}$ & $\begin{array}{c}\xi \mathrm{h} \\
{\left[\mathrm{cm}^{2} / \text { ton }\right]}\end{array}$ & $\begin{array}{c}\xi \mathrm{m} \\
{[\mathrm{cm} / \text { ton }]}\end{array}$ & $\begin{array}{c}\phi \mathrm{h} \\
{[\mathrm{cm} / \text { ton }]}\end{array}$ & $\begin{array}{c}\phi \mathrm{m} \\
{\left[\text { ton }^{-1}\right]}\end{array}$ \\
\hline 0,0015 & 192,3077 & 0,0371 & 12,7222 & 0,9442 & 0,9442 & 0,1401 \\
\hline
\end{tabular}

\begin{tabular}{|c|c|c|}
\hline $\begin{array}{c}\text { Mce } \\
{[\text { ton } \mathrm{cm} / \mathrm{cm}]}\end{array}$ & $\begin{array}{c}\text { Hce } \\
{[\text { ton } / \mathrm{cm}]}\end{array}$ & $\begin{array}{c}\text { Hca } \\
{[\text { ton } / \mathrm{cm}]}\end{array}$ \\
\hline 0,3444 & $-0,0526$ & $-0,0270$ \\
\hline
\end{tabular}

E para o comprimento ao longo do meridiano da casca necessário para o amortecimento das tensões de flexão causadas pela "perturbação de borda" medido a partir do vínculo :

$$
-\lambda_{\text {cone }}=170,46[\mathrm{~cm}]
$$

Na vertical chamou-se este valor de $\lambda z$ que é igual a $\lambda_{\text {cone. }} \operatorname{sen} \theta r=85,23[\mathrm{~cm}]$, e portanto a tabela de valores para a casca cônica deveria ser apresentada das 
coordenadas " $\mathrm{z}$ " igual a $\mathrm{H}-\lambda z=261,18$ até $\mathrm{H}=346,41$. Adotou-se de 259,81 até $346,41[\mathrm{~cm}]$.

\subsubsection{CASCA CÔNICA COM BORDA SUPERIOR ENGASTADA}

Para os deslocamentos radiais e rotações ao longo do meridiano da superfície média da casca cônica encontrou-se

TABELA 10 - Valores dos deslocamentos radiais $\xi \mathrm{e}(\mathrm{z})$ e rotações $\phi \mathrm{e}(\mathrm{z})$

\begin{tabular}{|c|c|c|c|c|c|c|}
\hline \multirow[b]{2}{*}{$\begin{array}{c}\mathrm{z} \\
{[\mathrm{cm}]}\end{array}$} & \multicolumn{2}{|c|}{$\begin{array}{l}\xi \mathrm{e}(\mathrm{z}) \\
{[\mathrm{cm}]}\end{array}$} & \multirow[b]{2}{*}{ Diferença } & \multicolumn{2}{|c|}{$\begin{array}{c}\phi \mathrm{e}(\mathrm{z}) \\
\text { [radianos] }\end{array}$} & \multirow[b]{2}{*}{ Diferença } \\
\hline & Teoria & $\begin{array}{c}\text { Elementos } \\
\text { Finitos }\end{array}$ & & Teoria & $\begin{array}{c}\text { Elementos } \\
\text { Finitos }\end{array}$ & \\
\hline 346,41 & 0,0000 & 0,0000 & 0,0000 & 0,0000 & 0,0000 & 0,0000 \\
\hline 343,52 & 0,0120 & 0,0116 & 0,0004 & $-0,0082$ & $-0,0084$ & 0,0002 \\
\hline 340,64 & 0,0424 & 0,0420 & 0,0004 & $-0,0129$ & $-0,0132$ & 0,0003 \\
\hline 337,75 & 0,0821 & 0,0823 & 0,0002 & $-0,0149$ & $-0,0152$ & 0,0003 \\
\hline 334,86 & 0,1247 & 0,1255 & 0,0008 & $-0,0149$ & $-0,0153$ & 0,0004 \\
\hline 331,98 & 0,1656 & 0,1671 & 0,0015 & $-0,0138$ & $-0,0141$ & 0,0003 \\
\hline 329,09 & 0,2020 & 0,2041 & 0,0021 & $-0,0119$ & $-0,0121$ & 0,0002 \\
\hline 326,20 & 0,2325 & 0,2349 & 0,0024 & $-0,0097$ & $-0,0098$ & 0,0001 \\
\hline 323,32 & 0,2565 & 0,2589 & 0,0024 & $-0,0075$ & $-0,0074$ & 0,0001 \\
\hline 320,43 & 0,2742 & 0,2762 & 0,0020 & $-0,0054$ & $-0,0052$ & 0,0002 \\
\hline 317,54 & 0,2861 & 0,2875 & 0,0014 & $-0,0037$ & $-0,0032$ & 0,0005 \\
\hline 314,66 & 0,2930 & 0,2937 & 0,0007 & $-0,0019$ & $-0,0016$ & 0,0003 \\
\hline 311,77 & 0,2958 & 0,2957 & 0,0001 & $-0,0006$ & $-0,0003$ & 0,0003 \\
\hline 308,88 & 0,2954 & 0,2945 & 0,0009 & 0,0003 & 0,0006 & 0,0003 \\
\hline 306,00 & 0,2926 & 0,2910 & 0,0016 & 0,0010 & 0,0013 & 0,0003 \\
\hline 303,11 & 0,2882 & 0,2858 & 0,0024 & 0,0015 & 0,0017 & 0,0002 \\
\hline 300,22 & 0,2826 & 0,2798 & 0,0028 & 0,0018 & 0,0019 & 0,0001 \\
\hline 297,34 & 0,2764 & 0,2733 & 0,0031 & 0,0020 & 0,0020 & 0,0000 \\
\hline 294,45 & 0,2700 & 0,2666 & 0,0034 & 0,0020 & 0,0020 & 0,0000 \\
\hline 291,56 & 0,2635 & 0,2601 & 0,0034 & 0,0020 & 0,0020 & 0,0000 \\
\hline 288,68 & 0,2571 & 0,2539 & 0,0032 & 0,0019 & 0,0019 & 0,0000 \\
\hline 285,79 & 0,2511 & 0,2480 & 0,0031 & 0,0018 & 0,0017 & 0,0001 \\
\hline 282,90 & 0,2453 & 0,2425 & 0,0028 & 0,0017 & 0,0016 & 0,0001 \\
\hline 280,01 & 0,2398 & 0,2372 & 0,0026 & 0,0016 & 0,0015 & 0,0001 \\
\hline 277,13 & 0,2346 & 0,2324 & 0,0023 & 0,0015 & 0,0014 & 0,0001 \\
\hline 274,24 & 0,2297 & 0,2277 & 0,0020 & 0,0014 & 0,0013 & 0,0001 \\
\hline 271,35 & 0,2250 & 0,2233 & 0,0017 & 0,0014 & 0,0013 & 0,0001 \\
\hline
\end{tabular}




\begin{tabular}{|c|r|r|r|}
\cline { 2 - 3 } \multicolumn{1}{c|}{} & \multicolumn{2}{c|}{\begin{tabular}{c}
\multicolumn{1}{c}{$(\mathrm{e}(\mathrm{z})$} \\
{$[\mathrm{cm}]$}
\end{tabular}} & \multicolumn{1}{c|}{} \\
\hline $\begin{array}{c}\mathrm{z} \\
{[\mathrm{cm}]}\end{array}$ & Teoria & $\begin{array}{c}\text { Elementos } \\
\text { Finitos }\end{array}$ & Diferença \\
\hline 268,47 & 0,2205 & 0,2190 & 0,0015 \\
\hline 265,58 & 0,2161 & 0,2148 & 0,0013 \\
\hline 262,69 & 0,2119 & 0,2107 & 0,0012 \\
\hline 259,81 & 0,2078 & 0,2067 & 0,0011 \\
\hline
\end{tabular}

\begin{tabular}{|c|c|r|}
\hline \multicolumn{2}{|c|}{\begin{tabular}{c}
\multicolumn{1}{c}{} \\
[radianos]
\end{tabular}} & \\
\hline Teoria & $\begin{array}{c}\text { Elementos } \\
\text { Finitos }\end{array}$ & Diferença \\
\hline 0,0013 & 0,0012 & 0,0001 \\
\hline 0,0013 & 0,0012 & 0,0001 \\
\hline 0,0012 & 0,0012 & 0,0000 \\
\hline 0,0012 & 0,0012 & 0,0000 \\
\hline
\end{tabular}

Teoria

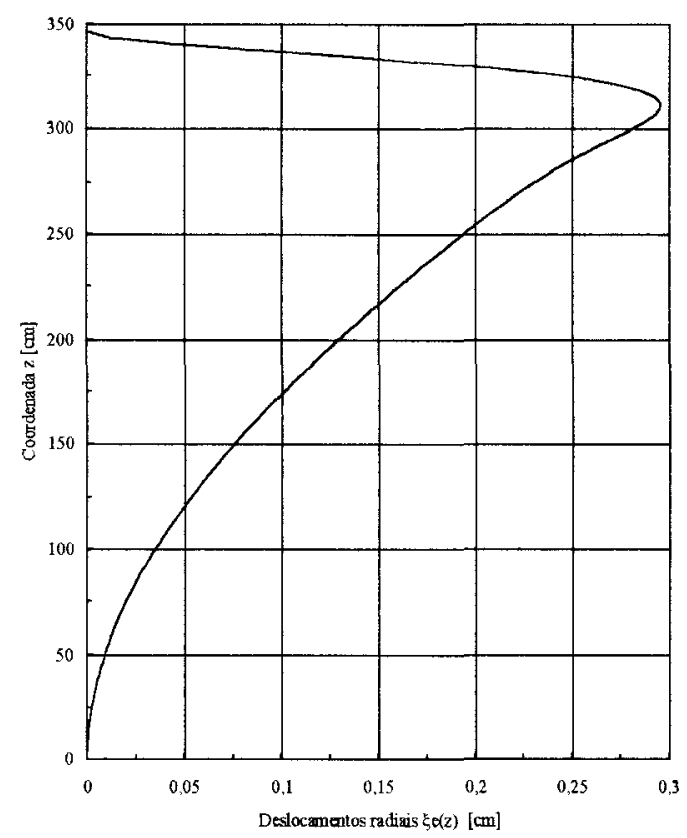

Elaneartos Finitos

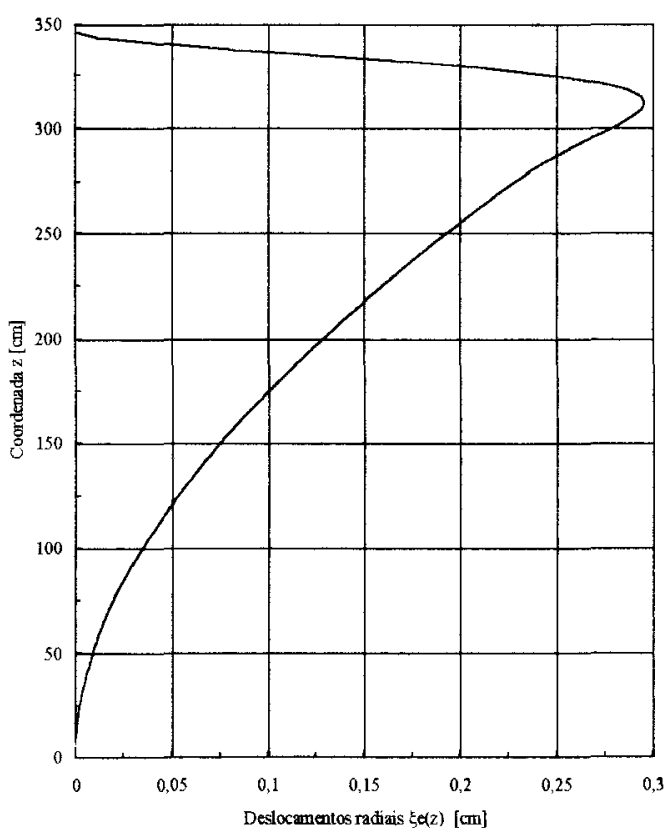

FIGURA 49 - Gráficos para os valores dos deslocamentos radiais $\xi \mathrm{e}(\mathrm{z})$ ao longo do meridiano da superficie média da casca cônica

Lembrando que a coluna "Diferença", da tabela, apresenta os valores das diferenças absolutas entre os parâmetros obtidos pelas equações da teoria aproximada e os obtidos pelo cálculo através do método dos elementos finitos. 

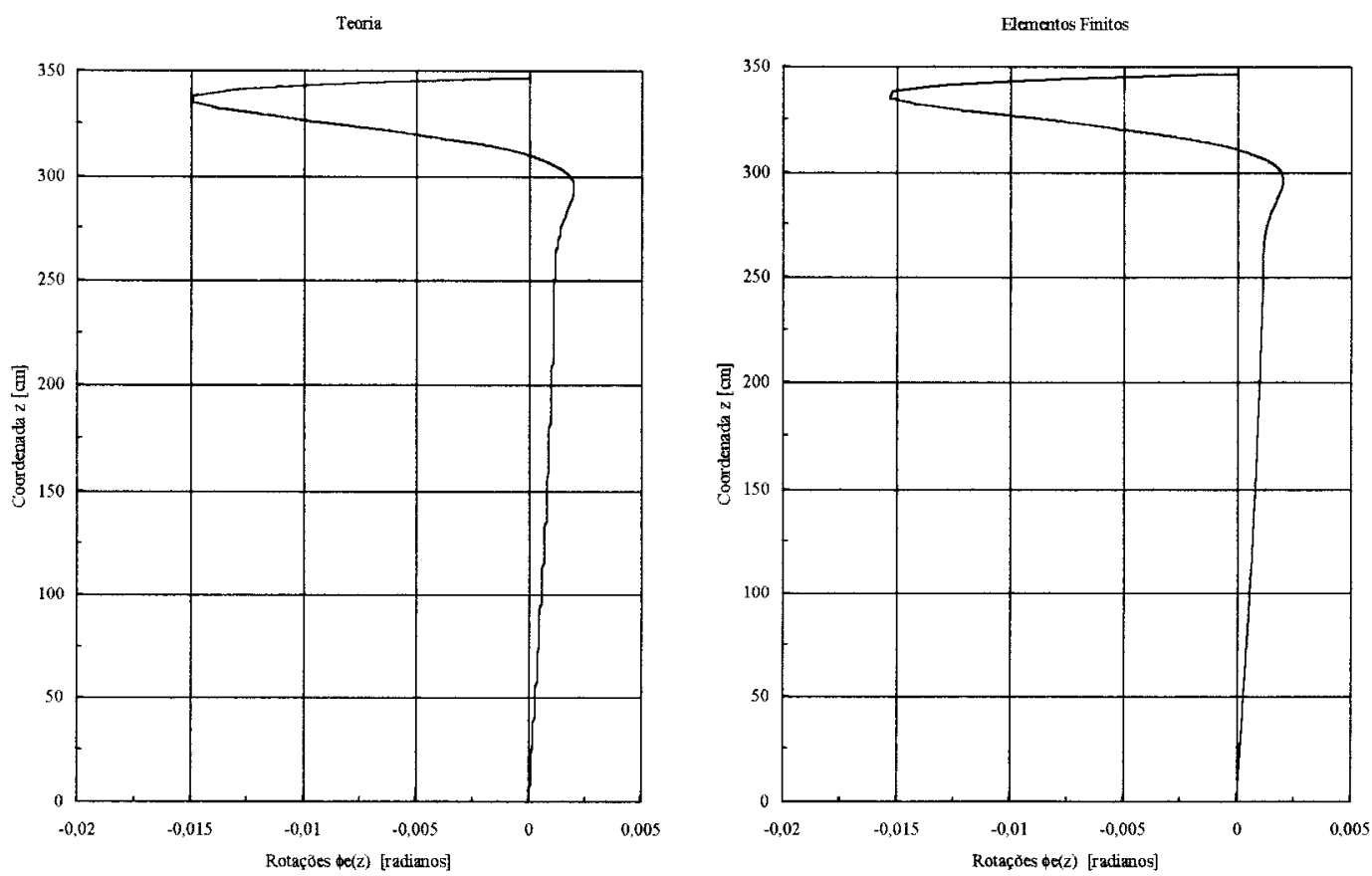

FIGURA 50 - Gráficos para os valores das rotações $\phi e(z)$ ao longo

do meridiano da superficie média da casca cônica

Os momentos solicitantes ao longo dos paralelos e dos meridianos da superficie média da casca cônica e suas correspondentes diferenças absolutas foram :

TABELA 11 - Valores dos momentos ao longo dos paralelos $\mathrm{M} 1 \mathrm{e}(\mathrm{z})$ e dos meridianos M2e(z)

\begin{tabular}{|c|r|r|r|}
\cline { 2 - 3 } \multicolumn{1}{c|}{} & \multicolumn{2}{c|}{\begin{tabular}{c}
\multicolumn{1}{c}{ Mle(z) } \\
[ton.cm/cm]
\end{tabular}} & \multicolumn{1}{c|}{} \\
\hline $\begin{array}{c}z \\
{[\mathrm{~cm}]}\end{array}$ & Teoria & $\begin{array}{c}\text { Elementos } \\
\text { Finitos }\end{array}$ & Diferença \\
\hline 346,41 & 0,3444 & 0,3471 & 0,0027 \\
\hline 343,52 & 0,2091 & 0,2107 & 0,0016 \\
\hline 340,64 & 0,1055 & 0,1054 & 0,0001 \\
\hline 337,75 & 0,0302 & 0,0281 & 0,0021 \\
\hline 334,86 & $-0,0211$ & $-0,0250$ & 0,0039 \\
\hline 331,98 & $-0,0530$ & $-0,0581$ & 0,0051 \\
\hline 329,09 & $-0,0698$ & $-0,0755$ & $\mathbf{0 , 0 0 5 6}$ \\
\hline 326,20 & $-0,0756$ & $-0,0811$ & 0,0055 \\
\hline 323,32 & $-0,0738$ & $-0,0785$ & 0,0047 \\
\hline 320,43 & $-0,0671$ & $-0,0707$ & 0,0036 \\
\hline
\end{tabular}

\begin{tabular}{|r|r|r|}
\hline \multicolumn{2}{|c|}{$\begin{array}{r}\text { M2e(z) } \\
\text { [ton.cm/cm] }\end{array}$} & \\
\cline { 1 - 2 } Teoria & $\begin{array}{c}\text { Elementos } \\
\text { Finitos }\end{array}$ & Diferença \\
\hline 0,1029 & 0,1041 & 0,0012 \\
\hline 0,0601 & 0,0611 & 0,0010 \\
\hline 0,0277 & 0,0283 & 0,0006 \\
\hline 0,0046 & 0,0045 & 0,0001 \\
\hline$-0,0109$ & $-0,0115$ & 0,0006 \\
\hline$-0,0201$ & $-0,0211$ & 0,0010 \\
\hline$-0,0246$ & $-0,0259$ & $\mathbf{0 , 0 0 1 3}$ \\
\hline$-0,0258$ & $-0,0269$ & 0,0011 \\
\hline$-0,0246$ & $-0,0256$ & 0,0010 \\
\hline$-0,0220$ & $-0,0226$ & 0,0006 \\
\hline
\end{tabular}




\begin{tabular}{|c|c|c|c|c|c|c|}
\hline \multirow[b]{2}{*}{$\begin{array}{c}\mathrm{z} \\
{[\mathrm{cm}]}\end{array}$} & \multicolumn{2}{|c|}{$\begin{array}{c}\mathrm{Mle}(\mathrm{z}) \\
\text { [ton } \mathrm{cm} / \mathrm{cm} \text { ] }\end{array}$} & \multirow[b]{2}{*}{ Diferença } & \multicolumn{2}{|c|}{$\begin{array}{c}\mathrm{M} 2 \mathrm{e}(\mathrm{z}) \\
{[\text { ton } \mathrm{cm} / \mathrm{cm}]}\end{array}$} & \multirow[b]{2}{*}{ Diferença } \\
\hline & Teoria & $\begin{array}{c}\text { Elementos } \\
\text { Finitos }\end{array}$ & & Teoria & $\begin{array}{c}\text { Elementos } \\
\text { Finitos }\end{array}$ & \\
\hline 317,54 & $-0,0578$ & $-0,0601$ & 0,0023 & $-0,0187$ & $-0,0189$ & 0,0002 \\
\hline 314,66 & $-0,0475$ & $-0,0485$ & 0,0010 & $-0,0151$ & $-0,0150$ & 0,0001 \\
\hline 311,77 & $-0,0372$ & $-0,0370$ & 0,0002 & $-0,0117$ & $-0,0112$ & 0,0005 \\
\hline 308,88 & $-0,0278$ & $-0,0266$ & 0,0012 & $-0,0086$ & $-0,0078$ & 0,0008 \\
\hline 306,00 & $-0,0196$ & $-0,0177$ & 0,0019 & $-0,0059$ & $-0,0049$ & 0,0010 \\
\hline 303,11 & $-0,0128$ & $-0,0104$ & 0,0024 & $-0,0038$ & $-0,0026$ & 0,0012 \\
\hline 300,22 & $-0,0074$ & $-0,0049$ & 0,0025 & $-0,0021$ & $-0,0009$ & 0,0012 \\
\hline 297,34 & $-0,0033$ & $-0,0009$ & 0,0024 & $-0,0008$ & 0,0003 & 0,0011 \\
\hline 294,45 & $-0,0005$ & 0,0018 & 0,0023 & 0,0001 & 0,0011 & 0,0010 \\
\hline 291,56 & 0,0015 & 0,0034 & 0,0019 & 0,0006 & 0,0016 & 0,0010 \\
\hline 288,68 & 0,0026 & 0,0042 & 0,0016 & 0,0010 & 0,0018 & 0,0008 \\
\hline 285,79 & 0,0031 & 0,0043 & 0,0012 & 0,0011 & 0,0018 & 0,0007 \\
\hline 282,90 & 0,0033 & 0,0041 & 0,0008 & 0,0011 & 0,0017 & 0,0006 \\
\hline 280,01 & 0,0031 & 0,0036 & 0,0005 & 0,0010 & 0,0016 & 0,0006 \\
\hline 277,13 & 0,0028 & 0,0030 & 0,0002 & 0,0009 & 0,0013 & 0,0004 \\
\hline 274,24 & 0,0024 & 0,0024 & 0,0000 & 0,0008 & 0,0011 & 0,0003 \\
\hline 271,35 & 0,0019 & 0,0019 & 0,0000 & 0,0006 & 0,0010 & 0,0004 \\
\hline 268,47 & 0,0015 & 0,0014 & 0,0001 & 0,0005 & 0,0008 & 0,0003 \\
\hline 265,58 & 0,0011 & 0,0010 & 0,0001 & 0,0003 & 0,0007 & 0,0004 \\
\hline 262,69 & 0,0007 & 0,0007 & 0,0000 & 0,0002 & 0,0006 & 0,0004 \\
\hline 259,81 & 0,0005 & 0,0005 & 0,0000 & 0,0001 & 0,0005 & 0,0004 \\
\hline
\end{tabular}



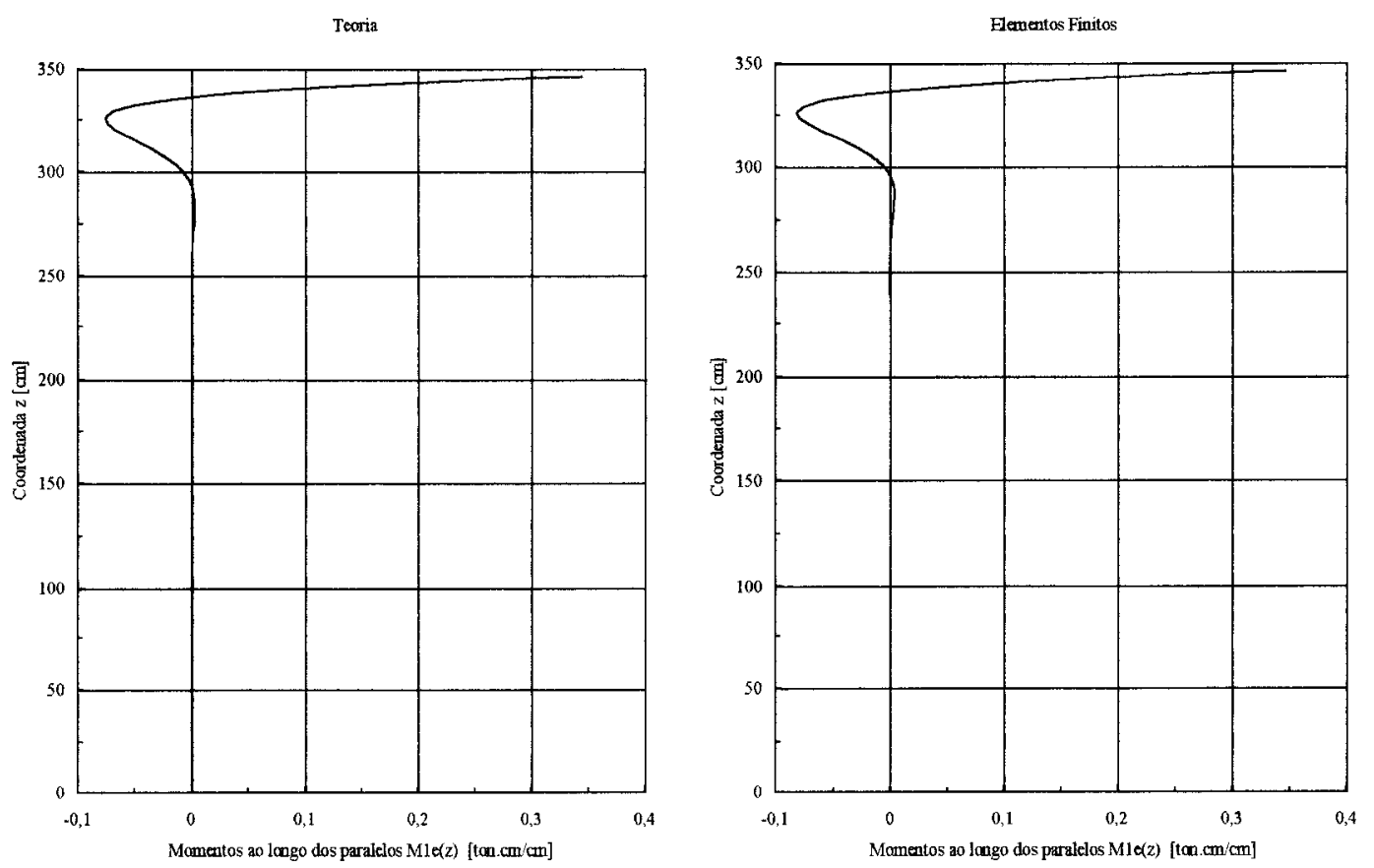

FIGURA 51 - Gráficos para os valores dos momentos ao longo dos paralelos $\mathrm{Mle}(\mathrm{z})$ da superficie média da casca cônica
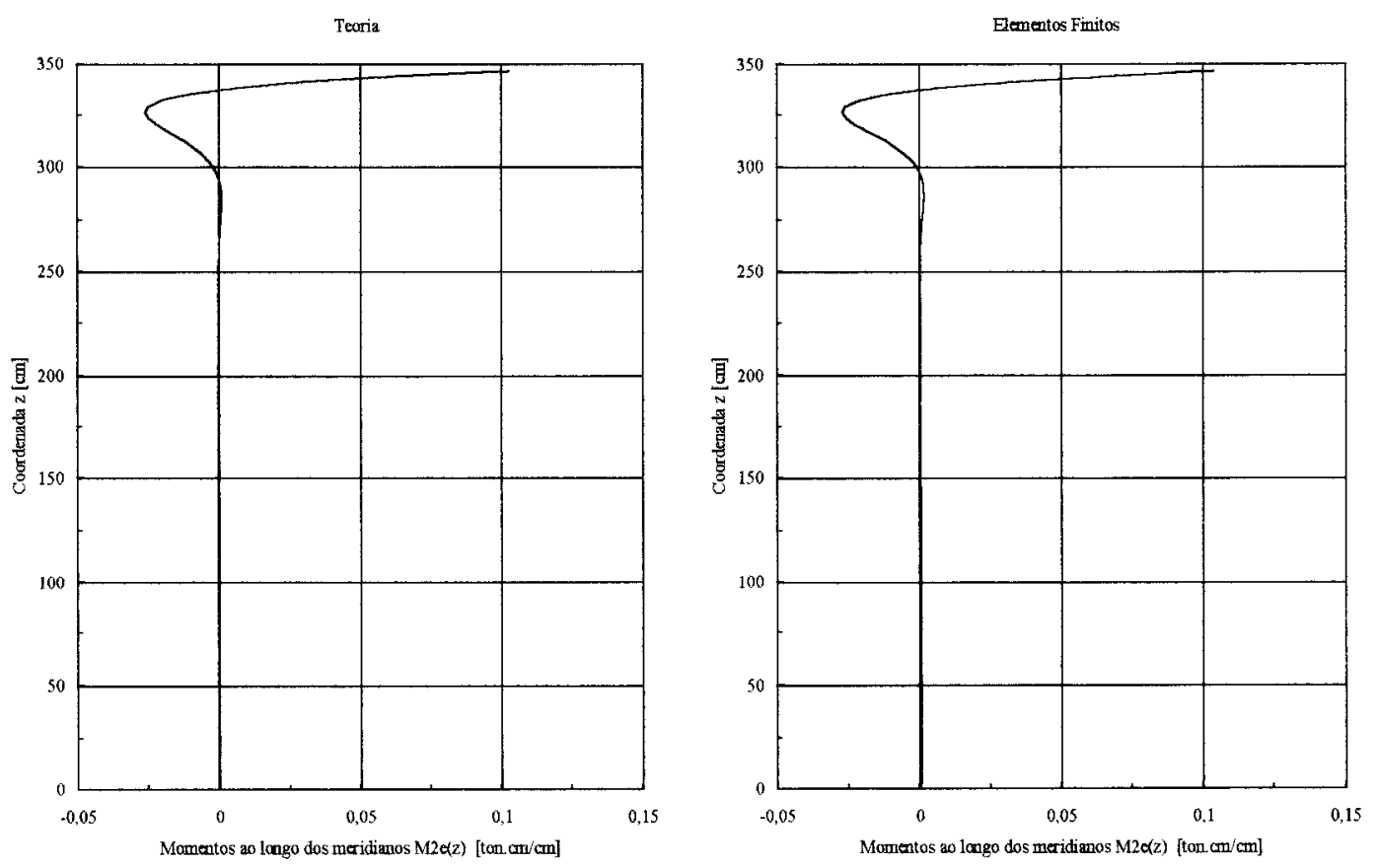

FIGURA 52 - Gráficos para os valores dos momentos ao longo dos meridianos $\mathrm{M} 2 \mathrm{e}(\mathrm{z})$ da superfície média da casca cônica 
Para as tensões atuantes ao longo dos meridianos da superficie interna e externa da casca cônica obteve-se :

TABELA 12 - Valores para as tensões ao longo dos meridianos da superficie interna $\sigma$ lein(z) e externa $\sigma$ leex(z) da casca

\begin{tabular}{|c|c|c|c|c|c|c|}
\hline \multirow[b]{2}{*}{$\begin{array}{c}\mathrm{z} \\
{[\mathrm{cm}]}\end{array}$} & \multicolumn{2}{|c|}{$\begin{array}{c}\sigma l \operatorname{ein}(\mathrm{z}) \\
{\left[\mathrm{ton} / \mathrm{cm}^{2}\right]}\end{array}$} & \multirow[b]{2}{*}{ Diferença } & \multicolumn{2}{|c|}{$\begin{array}{l}\sigma l \operatorname{eex}(\mathrm{z}) \\
{\left[\mathrm{ton} / \mathrm{cm}^{2}\right]}\end{array}$} & \multirow[b]{2}{*}{ Diferença } \\
\hline & Teoria & $\begin{array}{c}\text { Elementos } \\
\text { Finitos }\end{array}$ & & Teoria & $\begin{array}{c}\text { Elementos } \\
\text { Finitos }\end{array}$ & \\
\hline 346,41 & $-1,3226$ & $-1,3483$ & 0,0257 & 2,8101 & 2,8168 & 0,0067 \\
\hline 343,52 & $-0,5067$ & $-0,5270$ & 0,0203 & 2,0030 & 2,0017 & 0,0013 \\
\hline 340,64 & 0,1186 & 0,1109 & 0,0077 & 1,3849 & 1,3762 & 0,0088 \\
\hline 337,75 & 0,5729 & 0,5796 & 0,0067 & 0,9354 & 0,9166 & 0,0188 \\
\hline 334,86 & 0,8818 & 0,9010 & 0,0192 & 0,6286 & 0,6007 & 0,0279 \\
\hline 331,98 & 1,0726 & 1,1004 & 0,0278 & 0,4370 & 0,4032 & 0,0339 \\
\hline 329,09 & 1,1720 & 1,2037 & 0,0317 & 0,3344 & 0,2983 & 0,0361 \\
\hline 326,20 & 1,2040 & 1,2352 & 0,0312 & 0,2968 & 0,2624 & 0,0344 \\
\hline 323,32 & 1,1893 & 1,2164 & 0,0271 & 0,3040 & 0,2743 & 0,0297 \\
\hline 320,43 & 1,1449 & 1,1653 & 0,0203 & 0,3395 & 0,3164 & 0,0231 \\
\hline 317,54 & 1,0840 & 1,0964 & 0,0123 & 0,3902 & 0,3749 & 0,0153 \\
\hline 314,66 & 1,0164 & 1,0207 & 0,0042 & 0,4468 & 0,4391 & 0,0077 \\
\hline 311,77 & 0,9490 & 0,9458 & 0,0032 & 0,5026 & 0,5017 & 0,0009 \\
\hline 308,88 & 0,8864 & 0,8771 & 0,0093 & 0,5532 & 0,5579 & 0,0047 \\
\hline 306,00 & 0,8311 & 0,8172 & 0,0139 & 0,5964 & 0,6050 & 0,0086 \\
\hline 303,11 & 0,7842 & 0,7674 & 0,0168 & 0,6310 & 0,6420 & 0,0110 \\
\hline 300,22 & 0,7458 & 0,7278 & 0,0180 & 0,6571 & 0,6690 & 0,0119 \\
\hline 297,34 & 0,7154 & 0,6975 & 0,0179 & 0,6753 & 0,6868 & 0,0115 \\
\hline 294,45 & 0,6920 & 0,6752 & 0,0168 & 0,6865 & 0,6966 & 0,0101 \\
\hline 291,56 & 0,6745 & 0,6595 & 0,0150 & 0,6920 & 0,7001 & 0,0081 \\
\hline 288,68 & 0,6616 & 0,6489 & 0,0127 & 0,6928 & 0,6987 & 0,0059 \\
\hline 285,79 & 0,6524 & 0,6420 & 0,0104 & 0,6901 & 0,6938 & 0,0037 \\
\hline 282,90 & 0,6457 & 0,6375 & 0,0082 & 0,6849 & 0,6864 & 0,0015 \\
\hline 280,01 & 0,6407 & 0,6344 & 0,0063 & 0,6780 & 0,6777 & 0,0003 \\
\hline 277,13 & 0,6368 & 0,6321 & 0,0047 & 0,6701 & 0,6684 & 0,0017 \\
\hline 274,24 & 0,6335 & 0,6299 & 0,0036 & 0,6617 & 0,6589 & 0,0028 \\
\hline 271,35 & 0,6303 & 0,6274 & 0,0029 & 0,6531 & 0,6497 & 0,0034 \\
\hline 268,47 & 0,6269 & 0,6245 & 0,0024 & 0,6445 & 0,6409 & 0,0036 \\
\hline 265,58 & 0,6233 & 0,6210 & 0,0023 & 0,6362 & 0,6326 & 0,0036 \\
\hline 262,69 & 0,6194 & 0,6169 & 0,0025 & 0,6283 & 0,6249 & 0,0034 \\
\hline 259,81 & 0,6151 & 0,6122 & 0,0029 & 0,6207 & 0,6177 & 0,0030 \\
\hline
\end{tabular}



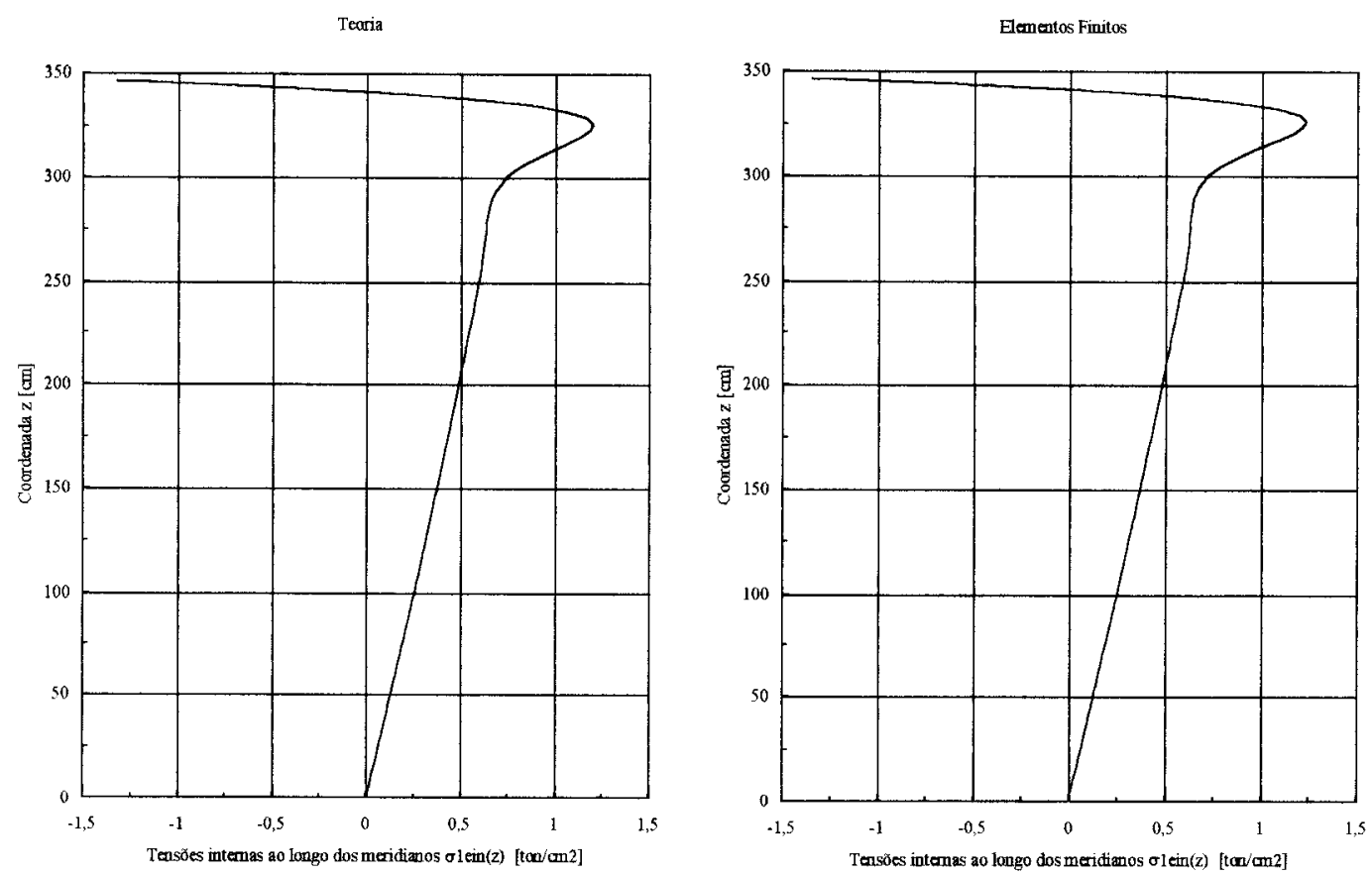

FIGURA 53 - Gráficos para os valores das tensões ao longo dos meridianos da superfície interna $\sigma$ lein(z) da casca cônica
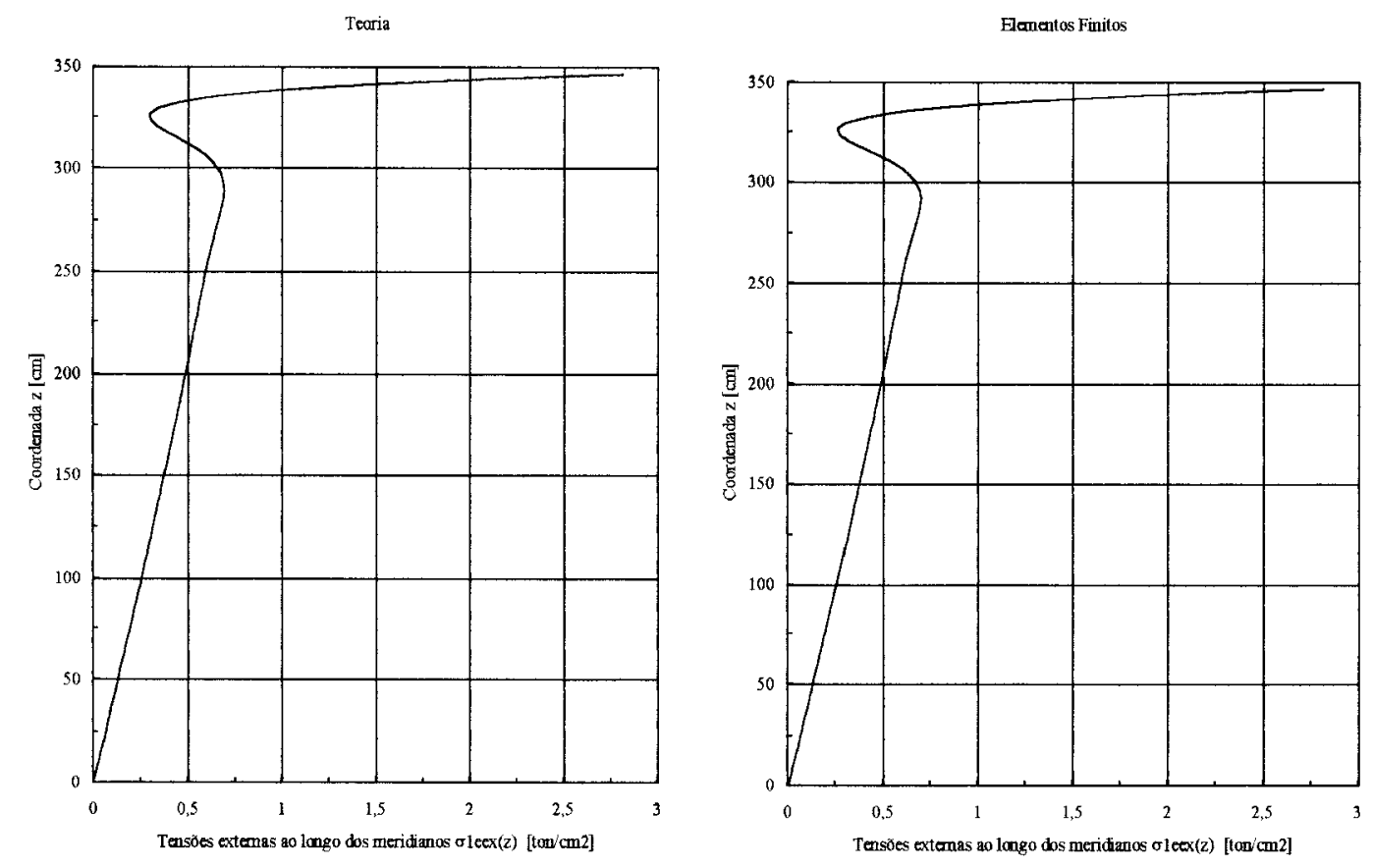

FIGURA 54 - Gráficos para os valores das tensões ao longo dos meridianos da superficie externa $\sigma$ leex $(\mathrm{z})$ da casca cônica 
E finalmente para as tensões atuantes ao longo dos paralelos da superficie interna e externa da casca cônica encontrou-se :

TABELA 13 - Valores para as tensões ao longo dos paralelos da superficie interna $\sigma 2 \operatorname{ein}(z)$ e externa $\sigma 2 \operatorname{eex}(z)$ da casca

\begin{tabular}{|c|c|c|c|c|c|c|}
\hline \multirow[b]{2}{*}{$\begin{array}{c}\mathrm{z} \\
{[\mathrm{cm}]}\end{array}$} & \multicolumn{2}{|c|}{$\begin{array}{c}\sigma 2 \operatorname{ein}(\mathrm{z}) \\
{\left[\mathrm{ton} / \mathrm{cm}^{2}\right]}\end{array}$} & \multirow[b]{2}{*}{ Diferença } & \multicolumn{2}{|c|}{$\begin{array}{l}\sigma 2 \operatorname{eex}(\mathrm{z}) \\
{\left[\mathrm{ton} / \mathrm{cm}^{2}\right]}\end{array}$} & \multirow[b]{2}{*}{ Diferença } \\
\hline & Teoria & $\begin{array}{c}\text { Elementos } \\
\text { Finitos }\end{array}$ & & Teoria & $\begin{array}{c}\text { Elementos } \\
\text { Finitos }\end{array}$ & \\
\hline 346,41 & $-0,3808$ & $-0,4045$ & 0,0237 & 0,8544 & 0,8450 & 0,0094 \\
\hline 343,52 & $-0,0733$ & $-0,1045$ & 0,0312 & 0,6476 & 0,6285 & 0,0191 \\
\hline 340,64 & 0,2355 & 0,2031 [ & 0,0324 & 0,5679 & 0,5421 & 0,0258 \\
\hline 337,75 & 0,5216 & 0,4928 & 0,0288 & 0,5763 & 0,5466 & 0,0297 \\
\hline 334,86 & 0,7710 & 0,7487 & 0,0223 & 0,6407 & 0,6107 & $\mathbf{0 , 0 3 0 0}$ \\
\hline 331,98 & 0,9774 & 0,9627 & 0,0147 & 0,7363 & 0,7090 & 0,0273 \\
\hline 329,09 & 1,1399 & 1,1324 & 0,0075 & 0,8443 & 0,8221 & 0,0222 \\
\hline 326,20 & 1,2609 & 1,2593 & 0,0016 & 0,9518 & 0,9359 & 0,0159 \\
\hline 323,32 & 1,3453 & 1,3477 & 0,0024 & 1,0503 & 1,0410 & 0,0094 \\
\hline 320,43 & 1,3990 & 1,4032 & 0,0042 & 1,1350 & 1,1315 & 0,0035 \\
\hline 317,54 & 1,4279 & 1,4322 & 0,0043 & 1,2037 & 1,2050 & 0,0013 \\
\hline 314,66 & 1,4379 & 1,4407 & 0,0028 & 1,2562 & 1,2608 & 0,0046 \\
\hline 311,77 & 1,4341 & 1,4344 & 0,0002 & 1,2936 & 1,3000 & 0,0064 \\
\hline 308,88 & 1,4209 & 1,4181 & 0,0028 & 1,3176 & 1,3245 & 0,0068 \\
\hline 306,00 & 1,4018 & 1,3959 & 0,0059 & 1,3305 & 1,3366 & 0,0061 \\
\hline 303,11 & 1,3797 & 1,3707 & 0,0090 & 1,3344 & 1,3390 & 0,0046 \\
\hline 300,22 & 1,3564 & 1,3449 & 0,0115 & 1,3315 & 1,3341 & 0,0026 \\
\hline 297,34 & 1,3334 & 1,3200 & 0,0134 & 1,3236 & 1,3240 & 0,0004 \\
\hline 294,45 & 1,3116 & 1,2969 & 0,0147 & 1,3124 & 1,3106 & 0,0018 \\
\hline 291,56 & 1,2915 & 1,2761 & 0,0155 & 1,2990 & 1,2953 & 0,0037 \\
\hline 288,68 & 1,2732 & 1,2577 & 0,0155 & 1,2846 & 1,2794 & 0,0053 \\
\hline 285,79 & 1,2567 & 1,2415 & 0,0152 & 1,2698 & 1,2634 & 0,0064 \\
\hline 282,90 & 1,2418 & 1,2274 & 0,0145 & 1,2551 & 1,2480 & 0,0071 \\
\hline 280,01 & 1,2284 & 1,2148 & 0,0136 & 1,2408 & 1,2334 & 0,0074 \\
\hline 277,13 & 1,2162 & 1,2035 & 0,0127 & 1,2271 & 1,2197 & 0,0074 \\
\hline 274,24 & 1,2049 & 1,1932 & 0,0118 & 1,2140 & 1,2069 & 0,0071 \\
\hline 271,35 & 1,1943 & 1,1834 & 0,0110 & 1,2015 & 1,1949 & 0,0066 \\
\hline 268,47 & 1,1842 & 1,1740 & 0,0102 & 1,1897 & 1,1836 & 0,0061 \\
\hline 265,58 & 1,1743 & 1,1647 & 0,0096 & 1,1783 & 1,1729 & 0,0054 \\
\hline 262,69 & 1,1646 & 1,1554 & 0,0093 & 1,1673 & 1,1625 & 0,0048 \\
\hline 259,81 & 1,1549 & 1,1460 & 0,0089 & 1,1566 & 1,1523 & 0,0043 \\
\hline
\end{tabular}



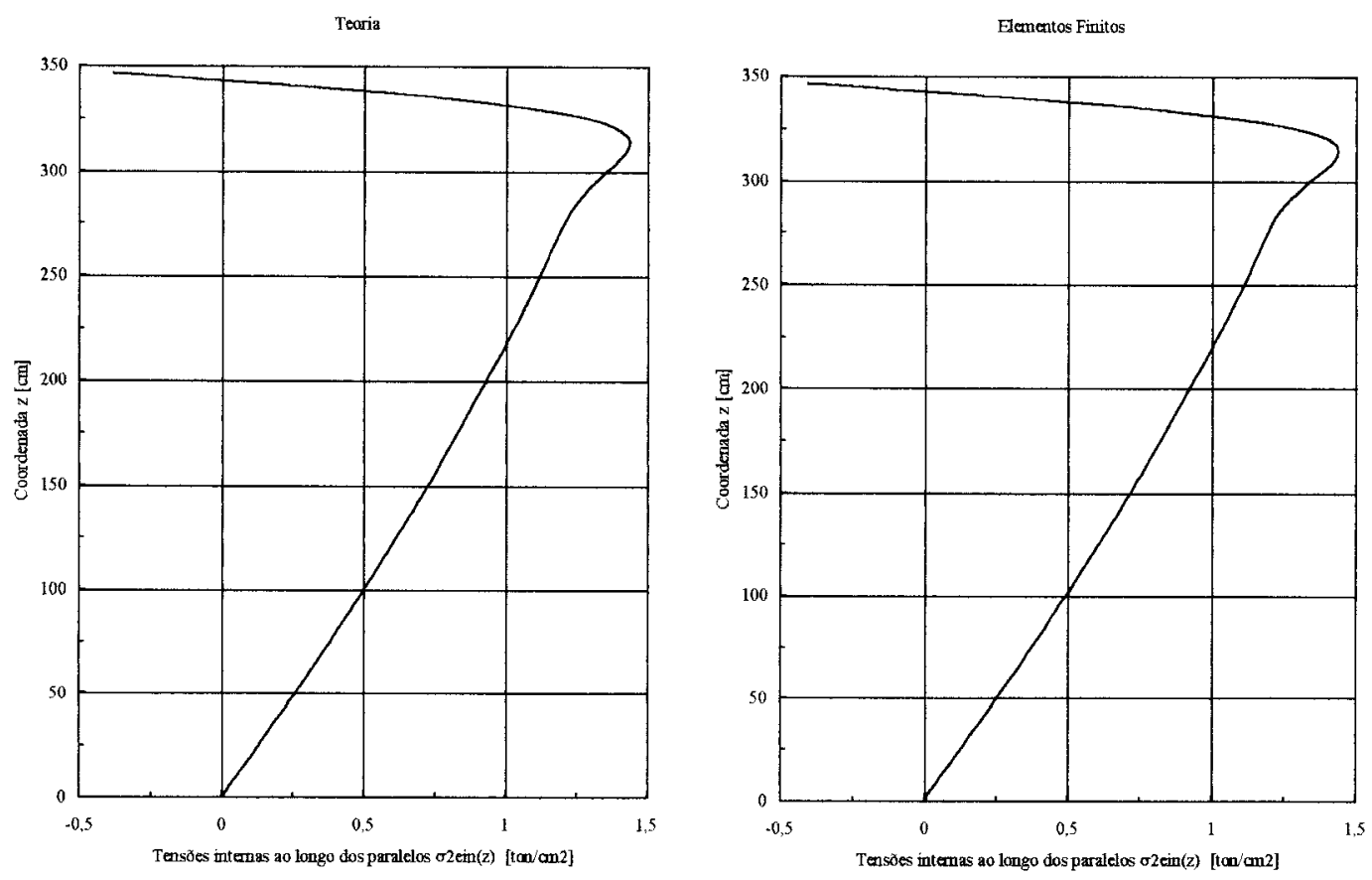

FIGURA 55 - Gráficos para os valores das tensões ao longo dos paralelos da superficie interna $\sigma 2 \operatorname{ein}(z)$ da casca cônica
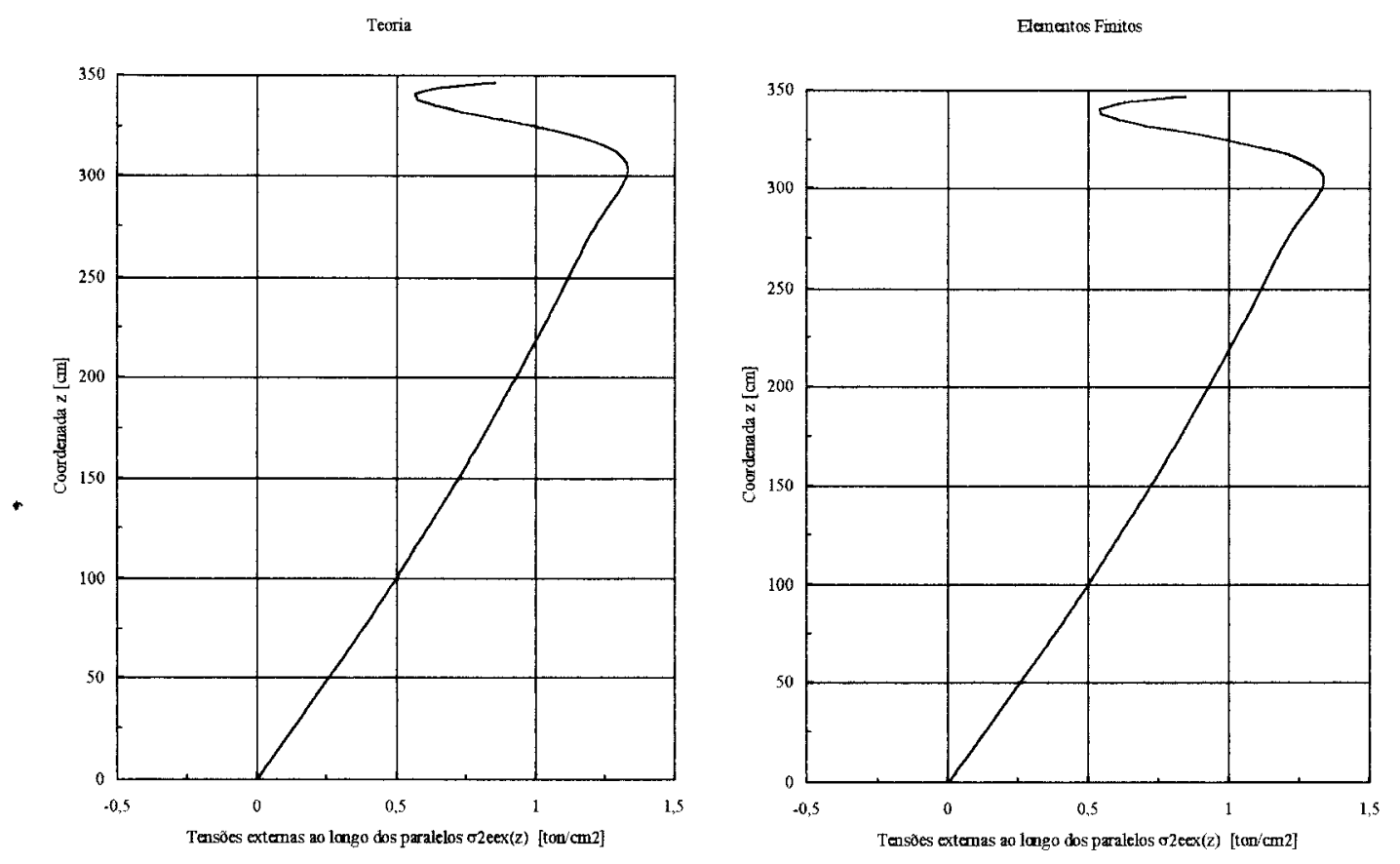

FIGURA 56 - Gráficos para os valores das tensões ao longo dos paralelos da superficie externa $\sigma 2 \operatorname{eex}(\mathrm{z})$ da casca cônica 
São apresentados abaixo os valores encontrados para as tensões de membrana atuantes ao longo dos meridianos e dos paralelos da superficie média da casca cônica para comparação :

TABELA 14 - Valores para as tensões teóricas de membrana ao longo dos meridianos $\sigma \operatorname{lm}(\mathrm{z})$ e paralelos $\sigma 2 \mathrm{~m}(\mathrm{z})$ da superficie média da casca

\begin{tabular}{|c|r|r|}
\hline $\begin{array}{c}\mathrm{z} \\
{[\mathrm{cm}]}\end{array}$ & $\begin{array}{c}\sigma 1 \mathrm{~m}(\mathrm{z}) \\
{\left[\text { ton } / \mathrm{cm}^{2}\right]}\end{array}$ & $\begin{array}{c}\sigma 2 \mathrm{~m}(\mathrm{z}) \\
{\left[\text { ton } / \mathrm{cm}^{2}\right]}\end{array}$ \\
\hline 346,41 & 0,7893 & 1,4400 \\
\hline 343,52 & 0,7838 & 1,4314 \\
\hline 340,64 & 0,7784 & 1,4228 \\
\hline 337,75 & 0,7729 & 1,4141 \\
\hline 334,86 & 0,7674 & 1,4054 \\
\hline 331,98 & 0,7619 & 1,3966 \\
\hline 329,09 & 0,7564 & 1,3877 \\
\hline 326,20 & 0,7509 & 1,3788 \\
\hline 323,32 & 0,7453 & 1,3699 \\
\hline 320,43 & 0,7397 & 1,3608 \\
\hline 317,54 & 0,7341 & 1,3518 \\
\hline 314,66 & 0,7285 & 1,3426 \\
\hline 311,77 & 0,7228 & 1,3334 \\
\hline 308,88 & 0,7172 & 1,3242 \\
\hline 306,00 & 0,7115 & 1,3148 \\
\hline 303,11 & 0,7058 & 1,3055 \\
\hline
\end{tabular}

\begin{tabular}{|r|r|r|}
\hline $\begin{array}{c}\mathrm{z} \\
{[\mathrm{cm}]}\end{array}$ & $\begin{array}{c}\sigma 1 \mathrm{~m}(\mathrm{z}) \\
{\left[\text { ton } / \mathrm{cm}^{2}\right]}\end{array}$ & $\begin{array}{c}\sigma 2 \mathrm{~m}(\mathrm{z}) \\
{\left[\text { ton } / \mathrm{cm}^{2}\right]}\end{array}$ \\
\hline 300,22 & 0,7001 & 1,2960 \\
\hline 297,34 & 0,6943 & 1,2865 \\
\hline 294,45 & 0,6886 & 1,2770 \\
\hline 291,56 & 0,6828 & 1,2674 \\
\hline 288,68 & 0,6770 & 1,2577 \\
\hline 285,79 & 0,6712 & 1,2480 \\
\hline 282,90 & 0,6653 & 1,2382 \\
\hline 280,01 & 0,6595 & 1,2284 \\
\hline 277,13 & 0,6536 & 1,2185 \\
\hline 274,24 & 0,6477 & 1,2086 \\
\hline 271,35 & 0,6418 & 1,1986 \\
\hline 268,47 & 0,6359 & 1,1885 \\
\hline 265,58 & 0,6299 & 1,1784 \\
\hline 262,69 & 0,6239 & 1,1682 \\
\hline 259,81 & 0,6179 & 1,1579 \\
\hline
\end{tabular}

Pode-se ver abaixo nos gráficos com as tensões teóricas ao longo dos meridianos e paralelos da casca cônica, que as mesmas possuem seus valores praticamente coincidêntes abaixo da região de $\mathrm{z}=\mathrm{H}-\lambda \mathrm{z}=261,18[\mathrm{~cm}]$. 
Teoria

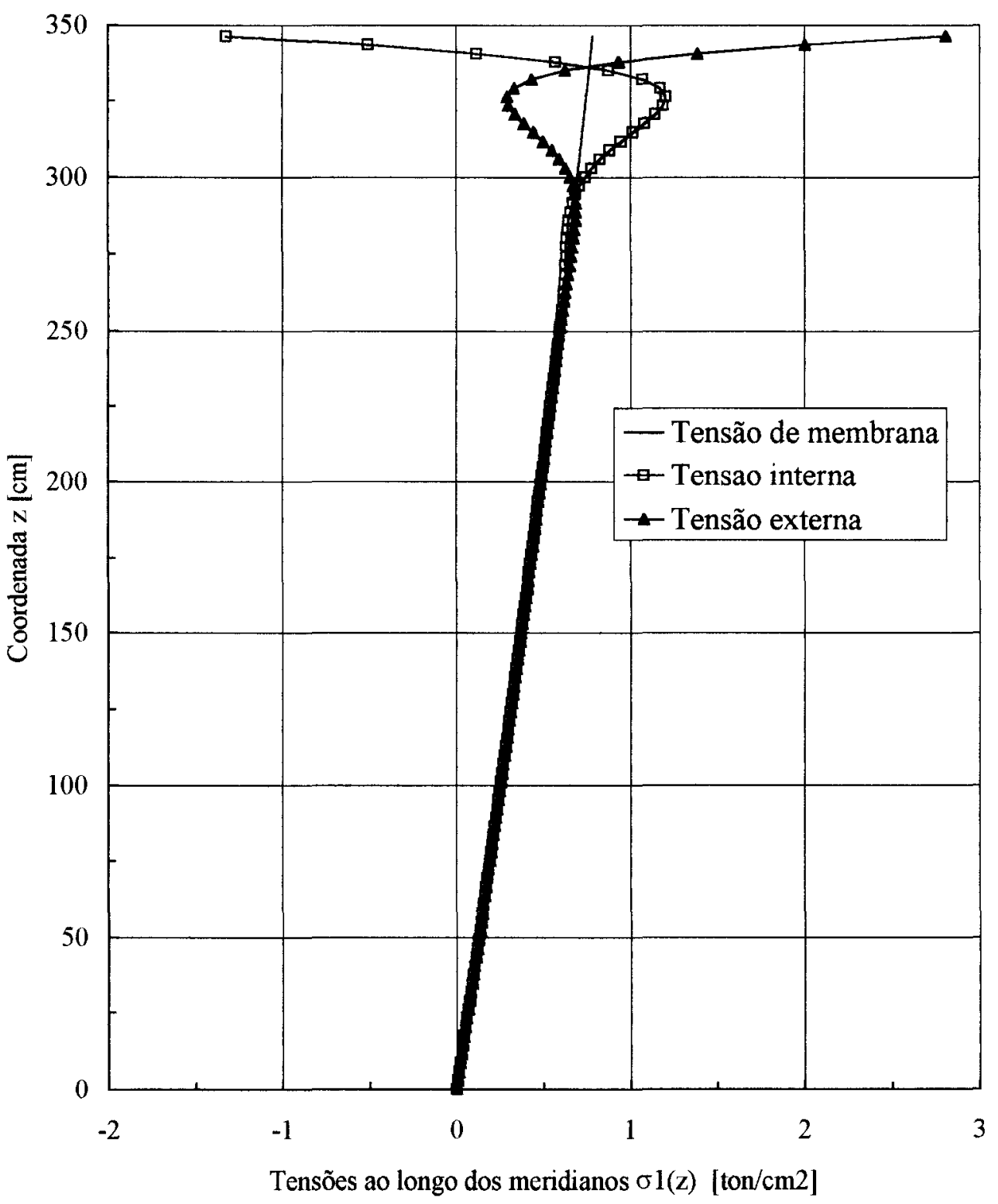

FIGURA 57 - Gráfico comparativo para os valores das tensões ao longo dos meridianos da casca cônica : $\sigma 1 \mathrm{~m}(\mathrm{z}), \sigma 1 \operatorname{ein}(\mathrm{z})$ e $\sigma \operatorname{leex}(\mathrm{z})$ 
Teoria

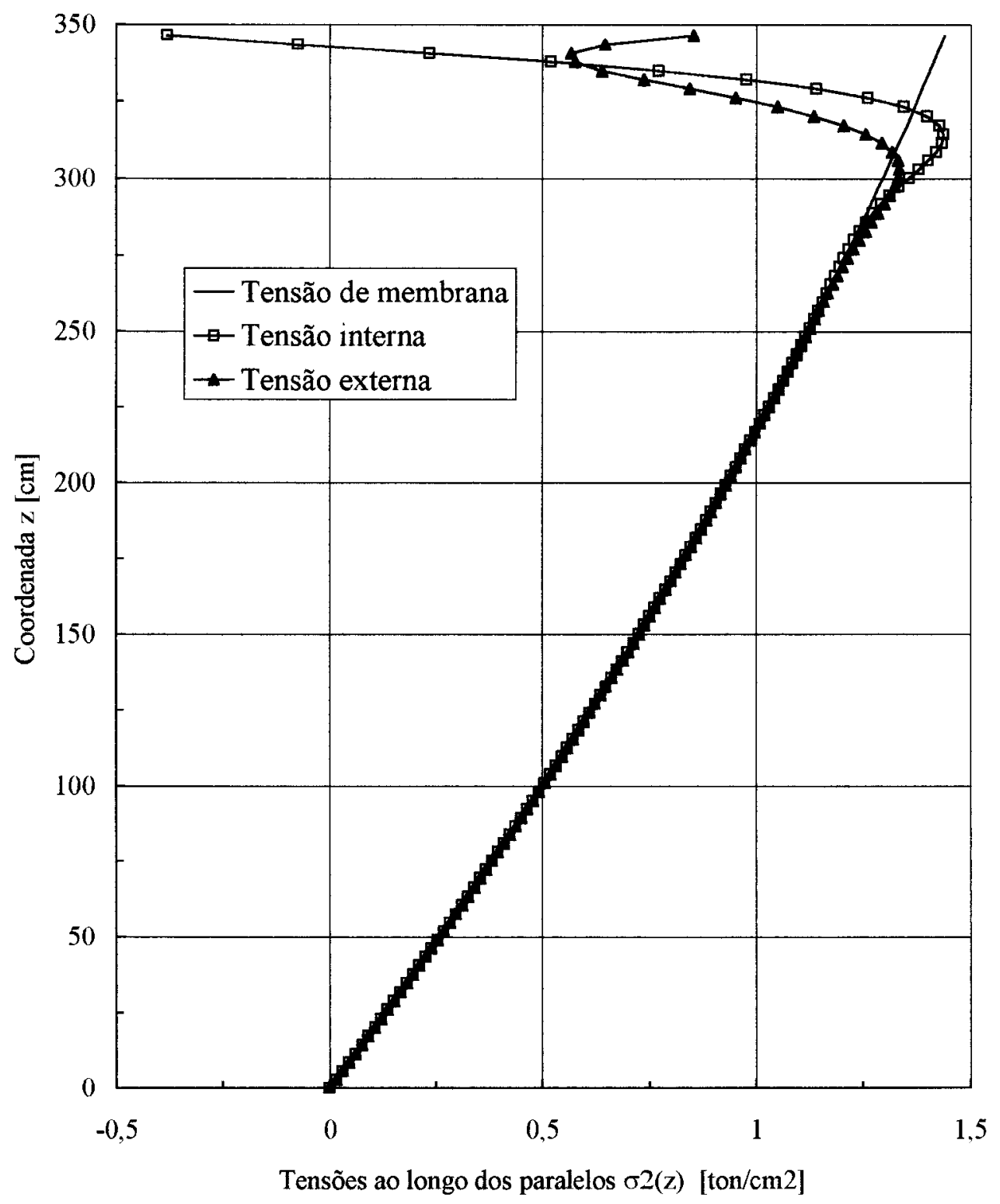

FIGURA 58 - Gráfico comparativo para os valores das tensões ao longo dos paralelos da casca cônica : $\sigma 2 \mathrm{~m}(\mathrm{z}), \sigma 2 \operatorname{ein}(\mathrm{z})$ e $\sigma 2 \operatorname{eex}(\mathrm{z})$ 


\subsubsection{CASCA CÔNICA COM BORDA SUPERIOR APOIADA FIXA}

Para os deslocamentos radiais e rotações ao longo do meridiano da superficie média da casca cônica encontrou-se :

TABELA 15 - Valores dos deslocamentos radiais $\xi \mathrm{a}(\mathrm{z})$ e rotações $\phi a(z)$

\begin{tabular}{|c|c|c|c|c|c|c|}
\hline \multirow[b]{2}{*}{$\begin{array}{c}\mathrm{z} \\
{[\mathrm{cm}]}\end{array}$} & \multicolumn{2}{|c|}{$\begin{array}{l}\xi \mathrm{a}(\mathrm{z}) \\
{[\mathrm{cm}]}\end{array}$} & \multirow[b]{2}{*}{ Diferença } & \multicolumn{2}{|c|}{$\begin{array}{c}\phi \mathrm{a}(\mathrm{z}) \\
{[\mathrm{radianos}]}\end{array}$} & \multirow[b]{2}{*}{ Diferença } \\
\hline & Teoria & \begin{tabular}{|c|c|} 
Elementos \\
Finitos
\end{tabular} & & Teoria & $\begin{array}{c}\text { Elementos } \\
\text { Finitos }\end{array}$ & \\
\hline 346,41 & 0,0000 & 0,0000 & 0,0000 & $-0,0241$ & $-0,0241$ & 0,0001 \\
\hline 343,52 & 0,0678 & 0,0674 & 0,0004 & $-0,0231$ & $-0,0232$ & 0,0001 \\
\hline 340,64 & 0,1304 & 0,1300 & 0,0004 & $-0,0207$ & $-0,0208$ & 0,0001 \\
\hline 337,75 & 0,1846 & 0,1845 & 0,0002 & $-0,0174$ & $-0,0175$ & 0,0001 \\
\hline 334,86 & 0,2290 & 0,2291 & 0,0000 & $-0,0139$ & $-0,0140$ & 0,0001 \\
\hline 331,98 & 0,2634 & 0,2635 & 0,0001 & $-0,0105$ & $-0,0105$ & 0,0000 \\
\hline 329,09 & 0,2883 & 0,2884 & 0,0001 & $-0,0074$ & $-0,0073$ & 0,0001 \\
\hline 326,20 & 0,3049 & 0,3047 & 0,0002 & $-0,0047$ & $-0,0046$ & 0,0001 \\
\hline 323,32 & 0,3145 & 0,3139 & 0,0006 & $-0,0025$ & $-0,0024$ & 0,0001 \\
\hline 320,43 & 0,3185 & 0,3173 & 0,0012 & $-0,0008$ & $-0,0006$ & 0,0002 \\
\hline 317,54 & 0,3182 & 0,3164 & 0,0018 & 0,0004 & 0,0007 & 0,0002 \\
\hline 314,66 & 0,3148 & 0,3124 & 0,0023 & 0,0013 & 0,0015 & 0,0002 \\
\hline 311,77 & 0,3092 & 0,3064 & 0,0028 & 0,0019 & 0,0021 & 0,0002 \\
\hline 308,88 & 0,3024 & 0,2992 & 0,0032 & 0,0022 & 0,0024 & 0,0001 \\
\hline 306,00 & 0,2949 & 0,2914 & 0,0035 & 0,0024 & 0,0025 & 0,0001 \\
\hline 303,11 & 0,2872 & $0,2836]$ & 0,0036 & 0,0024 & 0,0024 & 0,0000 \\
\hline 300,22 & 0,2796 & 0,2760 & 0,0036 & 0,0023 & 0,0023 & 0,0000 \\
\hline 297,34 & 0,2723 & 0,2689 & 0,0034 & 0,0022 & 0,0021 & 0,0001 \\
\hline 294,45 & 0,2655 & 0,2622 & 0,0032 & 0,0021 & 0,0020 & 0,0001 \\
\hline 291,56 & 0,2590 & 0,2561 & 0,0030 & 0,0019 & 0,0018 & 0,0001 \\
\hline 288,68 & 0,2531 & 0,2504 & 0,0026 & 0,0017 & 0,0016 & 0,0001 \\
\hline 285,79 & 0,2475 & 0,2452 & 0,0023 & 0,0016 & 0,0015 & 0,0001 \\
\hline 282,90 & 0,2423 & 0,2403 & 0,0020 & 0,0015 & 0,0014 & 0,0001 \\
\hline 280,01 & 0,2375 & 0,2357 & 0,0017 & 0,0014 & 0,0013 & 0,0001 \\
\hline 277,13 & 0,2329 & 0,2313 & 0,0015 & 0,0013 & 0,0012 & 0,0001 \\
\hline 274,24 & 0,2284 & 0,2271 & 0,0013 & 0,0013 & 0,0012 & 0,0001 \\
\hline 271,35 & 0,2242 & 0,2230 & 0,0012 & 0,0012 & 0,0012 & 0,0001 \\
\hline 268,47 & 0,2200 & 0,2189 & 0,0011 & 0,0012 & 0,0012 & 0,0000 \\
\hline 265,58 & 0,2159 & 0,2149 & 0,0010 & 0,0012 & 0,0012 & 0,0000 \\
\hline 262,69 & 0,2119 & 0,2109 & 0,0010 & 0,0012 & 0,0012 & 0,0000 \\
\hline 259,81 & 0,2079 & 0,2069 & 0,0010 & 0,0012 & 0,0012 & 0,0000 \\
\hline
\end{tabular}



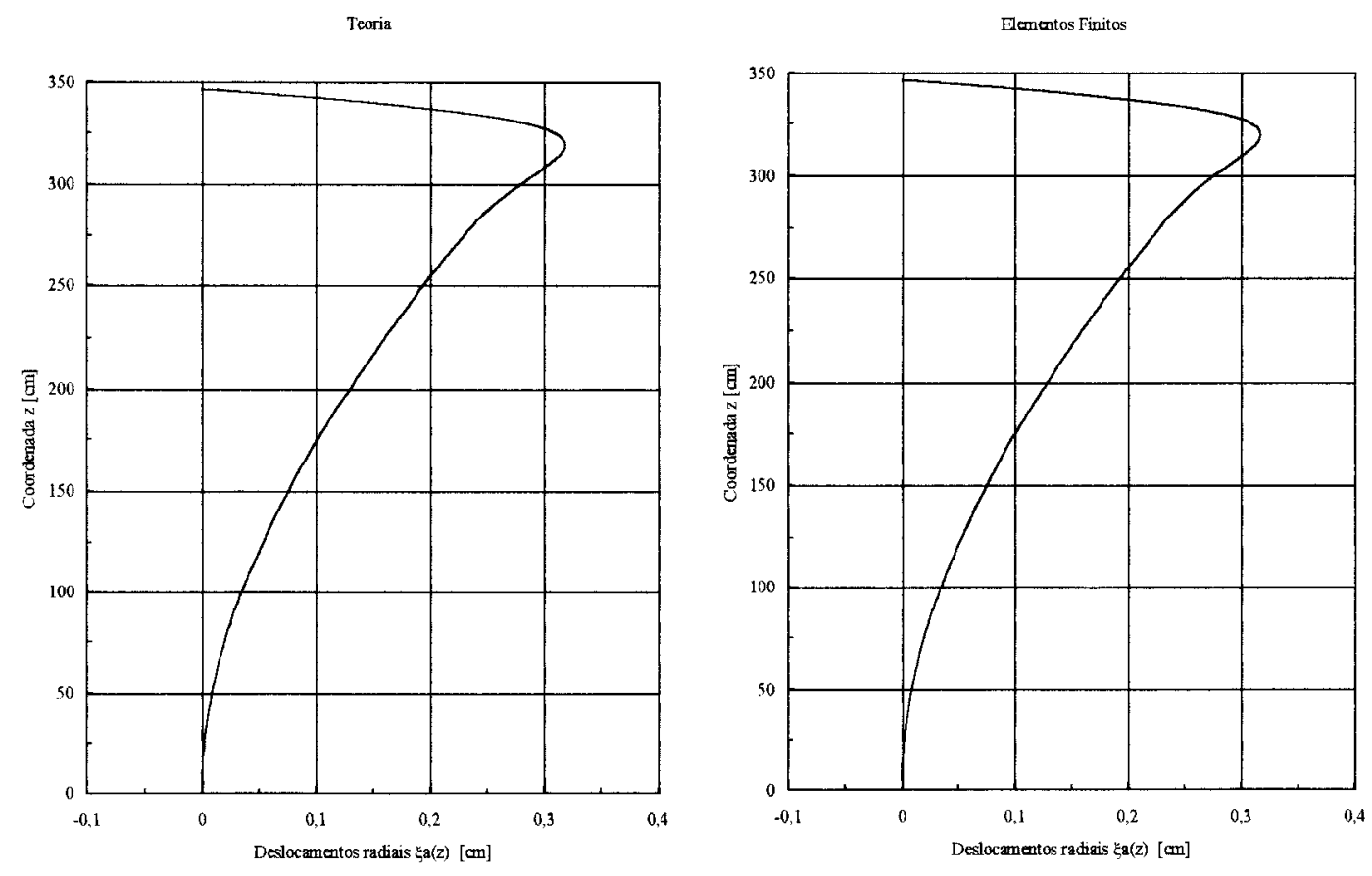

FIGURA 59 - Gráficos para os valores dos deslocamentos radiais $\xi \mathrm{a}(\mathrm{z})$ ao longo do meridiano da superfície média da casca cônica
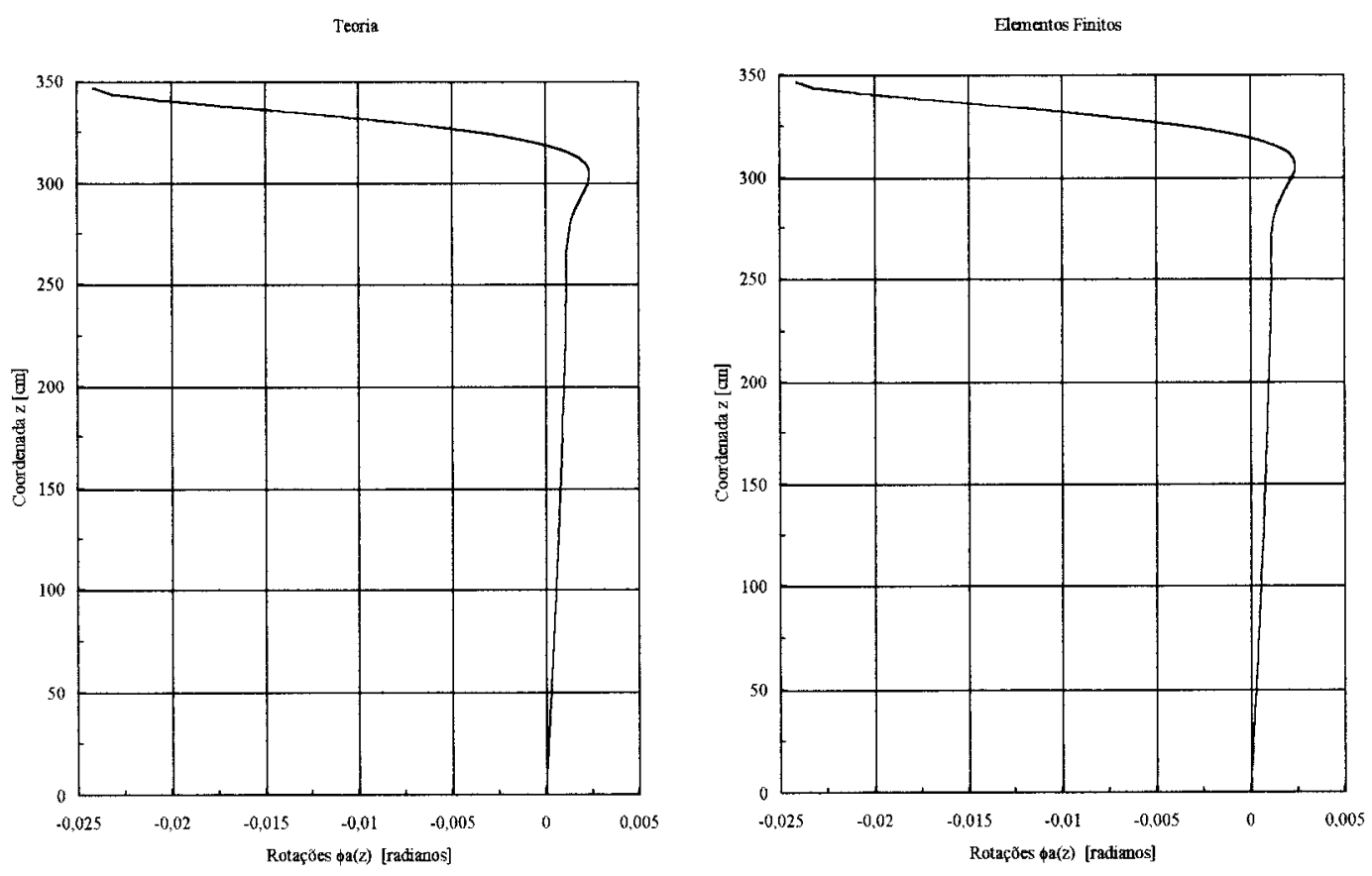

FIGURA 60 - Gráficos para os valores das rotações $\phi \mathrm{a}(\mathrm{z})$ ao longo do meridiano da superfície média da casca cônica 
Os momentos solicitantes ao longo dos paralelos e dos meridianos da superficie média da casca cônica e suas correspondentes diferenças absolutas foram

TABELA 16 - Valores dos momentos ao longo dos paralelos

$\mathrm{M} 1 \mathrm{a}(\mathrm{z})$ e dos meridianos M2a(z)

\begin{tabular}{|c|c|c|c|c|c|c|}
\hline \multirow[b]{2}{*}{$\begin{array}{c}\mathrm{z} \\
{[\mathrm{cm}]}\end{array}$} & \multicolumn{2}{|c|}{$\begin{array}{c}\mathrm{Mla}(\mathrm{z}) \\
{[\text { ton. } \mathrm{cm} / \mathrm{cm} \text { ] }}\end{array}$} & \multirow[b]{2}{*}{ Diferença } & \multicolumn{2}{|c|}{$\begin{array}{c}\mathrm{M} 2 \mathrm{a}(\mathrm{z}) \\
{[\text { ton } \mathrm{cm} / \mathrm{cm}]}\end{array}$} & \multirow[b]{2}{*}{ Diferença } \\
\hline & Teoria & $\begin{array}{c}\text { Elementos } \\
\text { Finitos }\end{array}$ & & Teoria & $\begin{array}{c}\text { Elementos } \\
\text { Finitos }\end{array}$ & \\
\hline 346,41 & 0,0000 & 0,0005 & 0,0005 & $-0,0071$ & $-0,0059$ & 0,0011 \\
\hline 343,52 & $-0,0625$ & $-0,0636$ & 0,0011 & $-0,0255$ & $-0,0250$ & 0,0006 \\
\hline 340,64 & $-0,0986$ & $-0,1009$ & 0,0023 & $-0,0357$ & $-0,0356$ & 0,0001 \\
\hline 337,75 & $-0,1148$ & $-0,1181$ & 0,0034 & $-0,0396$ & $-0,0400$ & 0,0003 \\
\hline 334,86 & $-0,1168$ & $-0,1207$ & 0,0039 & $-0,0393$ & $-0,0399$ & 0,0006 \\
\hline 331,98 & $-0,1095$ & $-0,1133$ & 0,0038 & $-0,0361$ & $-0,0368$ & 0,0006 \\
\hline 329,09 & $-0,0966$ & $-0,0998$ & 0,0032 & $-0,0314$ & $-0,0319$ & 0,0005 \\
\hline 326,20 & $-0,0811$ & $-0,0834$ & 0,0023 & $-0,0260$ & $-0,0263$ & 0,0002 \\
\hline 323,32 & $-0,0649$ & $-0,0662$ & 0,0013 & $-0,0206$ & $-0,0205$ & 0,0001 \\
\hline 320,43 & $-0,0496$ & $-0,0498$ & 0,0002 & $-0,0155$ & $-0,0151$ & 0,0004 \\
\hline 317,54 & $-0,0359$ & $-0,0352$ & 0,0007 & $-0,0110$ & $-0,0104$ & 0,0007 \\
\hline 314,66 & $-0,0244$ & $-0,0229$ & 0,0015 & $-0,0073$ & $-0,0064$ & 0,0009 \\
\hline 311,77 & $-0,0150$ & $-0,0130$ & 0,0020 & $-0,0044$ & $-0,0033$ & 0,0010 \\
\hline 308,88 & $-0,0078$ & $-0,0056$ & 0,0023 & $-0,0021$ & $-0,0010$ & 0,0011 \\
\hline 306,00 & $-0,0026$ & $-0,0003$ & 0,0023 & $-0,0005$ & 0,0006 & 0,0011 \\
\hline 303,11 & 0,0011 & 0,0032 & 0,0022 & 0,0006 & 0,0017 & 0,0010 \\
\hline 300,22 & 0,0033 & 0,0052 & 0,0019 & 0,0013 & 0,0022 & 0,0009 \\
\hline 297,34 & 0,0046 & 0,0061 & 0,0015 & 0,0016 & 0,0025 & 0,0008 \\
\hline 294,45 & 0,0050 & 0,0062 & 0,0012 & 0,0017 & 0,0024 & 0,0007 \\
\hline 291,56 & 0,0050 & 0,0058 & 0,0008 & 0,0017 & 0,0023 & 0,0006 \\
\hline 288,68 & 0,0046 & 0,0050 & 0,0005 & 0,0015 & 0,0020 & 0,0005 \\
\hline 285,79 & 0,0040 & 0,0042 & 0,0002 & 0,0013 & 0,0017 & 0,0004 \\
\hline 282,90 & 0,0033 & 0,0033 & 0,0000 & 0,0010 & 0,0014 & 0,0004 \\
\hline 280,01 & 0,0026 & 0,0025 & 0,0001 & 0,0008 & 0,0011 & 0,0003 \\
\hline 277,13 & 0,0019 & 0,0018 & 0,0002 & 0,0006 & 0,0009 & 0,0003 \\
\hline 274,24 & 0,0014 & 0,0012 & 0,0002 & 0,0004 & 0,0007 & 0,0003 \\
\hline 271,35 & 0,0009 & 0,0008 & 0,0001 & 0,0003 & 0,0006 & 0,0003 \\
\hline 268,47 & 0,0005 & 0,0005 & 0,0001 & 0,0002 & 0,0005 & 0,0004 \\
\hline 265,58 & 0,0003 & 0,0003 & 0,0000 & 0,0001 & 0,0005 & 0,0004 \\
\hline 262,69 & 0,0001 & 0,0002 & 0,0001 & 0,0000 & 0,0004 & 0,0004 \\
\hline 259,81 & $-0,0001$ & 0,0001 & 0,0002 & 0,0000 & 0,0004 & 0,0005 \\
\hline
\end{tabular}



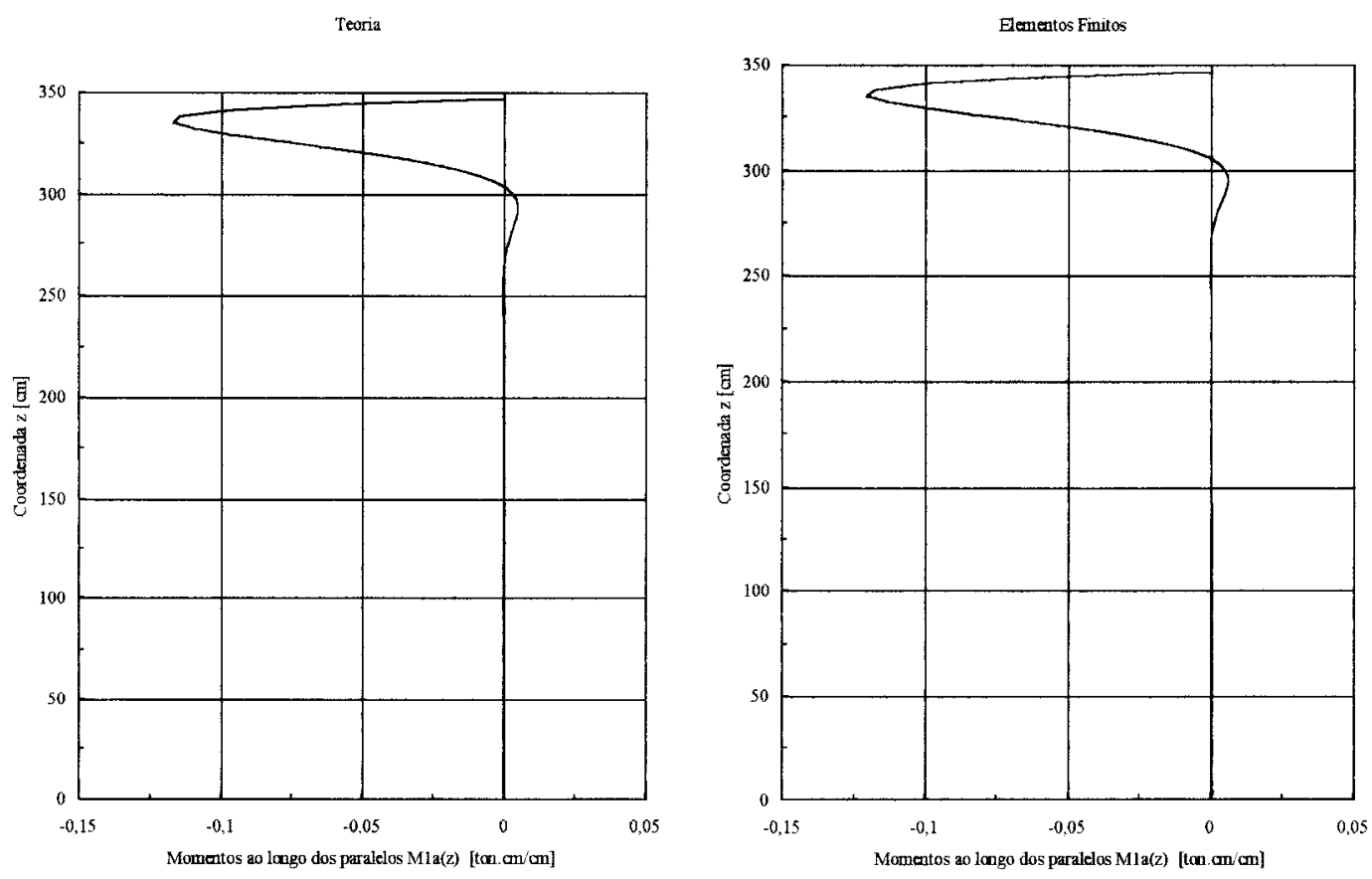

FIGURA 61 - Gráficos para os valores dos momentos ao longo dos paralelos Mla(z) da superfície média da casca cônica
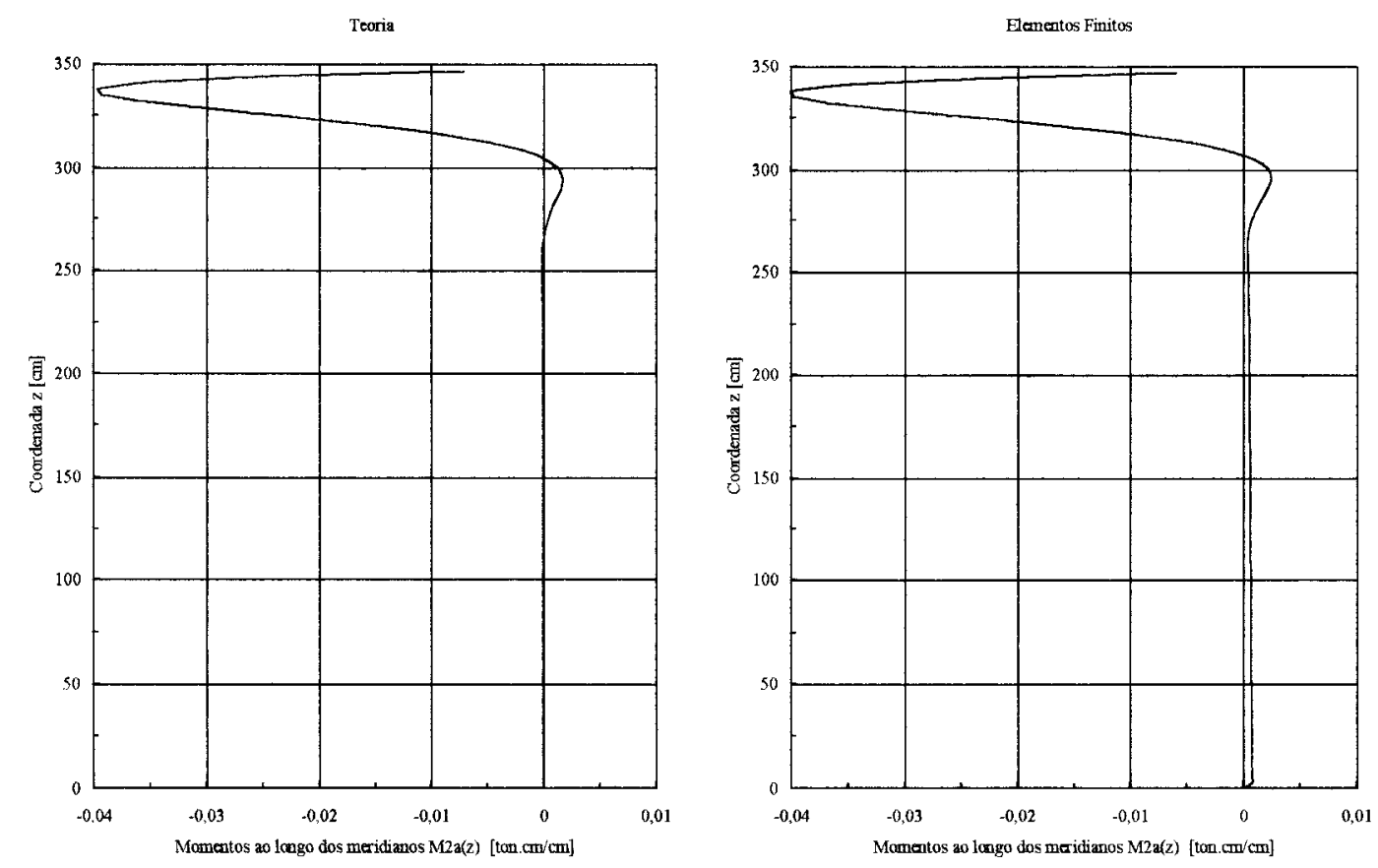

FIGURA 62 - Gráficos para os valores dos momentos ao longo dos meridianos 
M2a(z) da superfície média da casca cônica

Para as tensões atuantes ao longo dos meridianos da superficie interna e externa da casca cônica obteve-se :

TABELA 17 - Valores para as tensões ao longo dos meridianos da superficie interna $\sigma$ lain(z) e externa $\sigma$ laex(z) da casca

\begin{tabular}{|c|c|c|c|c|c|c|}
\hline \multirow[b]{2}{*}{$\begin{array}{c}\mathrm{z} \\
{[\mathrm{cm}]}\end{array}$} & \multicolumn{2}{|c|}{$\begin{array}{c}\sigma l \operatorname{ain}(\mathrm{z}) \\
{\left[\text { ton } / \mathrm{cm}^{2}\right]}\end{array}$} & \multirow[b]{2}{*}{ Diferença } & \multicolumn{2}{|c|}{$\begin{array}{l}\sigma l \mathrm{aex}(\mathrm{z}) \\
{\left[\operatorname{ton} / \mathrm{cm}^{2}\right]}\end{array}$} & \multirow[b]{2}{*}{ Diferença } \\
\hline & Teoria & $\begin{array}{c}\text { Elementos } \\
\text { Finitos }\end{array}$ & & Teoria & $\begin{array}{c}\text { Elementos } \\
\text { Finitos }\end{array}$ & \\
\hline 346,41 & 0,7659 & 0,7214 & 0,0445 & 0,7659 & 0,7269 & 0,0390 \\
\hline 343,52 & 1,1443 & 1,1471 & 0,0028 & 0,3945 & 0,3844 & 0,0101 \\
\hline 340,64 & 1,3622 & 1,3739 & 0,0117 & 0,1795 & 0,1631 & 0,0164 \\
\hline 337,75 & 1,4590 & 1,4776 & 0,0186 & 0,0819 & 0,0603 & 0,0216 \\
\hline 334,86 & 1,4693 & 1,4914 & 0,0221 & 0,0676 & 0,0434 & 0,0241 \\
\hline 331,98 & 1,4221 & 1,4440 & 0,0218 & 0,1081 & 0,0846 & 0,0235 \\
\hline 329,09 & 1,3405 & 1,3590 & 0,0185 & 0,1811 & 0,1609 & 0,0202 \\
\hline 326,20 & 1,2421 & 1,2550 & 0,0129 & 0,2693 & 0,2542 & 0,0151 \\
\hline 323,32 & 1,1396 & 1,1459 & 0,0063 & 0,3605 & 0,3514 & 0,0090 \\
\hline 320,43 & 1,0417 & 1,0412 & 0,0006 & 0,4464 & 0,4434 & 0,0030 \\
\hline 317,54 & 0,9536 & 0,9469 & 0,0066 & 0,5222 & 0,5245 & 0,0023 \\
\hline 314,66 & 0,8779 & 0,8664 & 0,0115 & 0,5853 & 0,5917 & 0,0064 \\
\hline 311,77 & 0,8156 & 0,8007 & 0,0148 & 0,6350 & 0,6442 & 0,0091 \\
\hline 308,88 & 0,7661 & 0,7494 & 0,0166 & 0,6720 & 0,6824 & 0,0104 \\
\hline 306,00 & 0,7282 & 0,7112 & 0,0171 & 0,6974 & 0,7078 & 0,0105 \\
\hline 303,11 & 0,7003 & 0,6840 & 0,0164 & 0,7130 & 0,7225 & 0,0095 \\
\hline 300,22 & 0,6806 & 0,6657 & 0,0148 & 0,7206 & 0,7284 & 0,0078 \\
\hline 297,34 & 0,6671 & 0,6543 & 0,0128 & 0,7220 & 0,7277 & 0,0057 \\
\hline 294,45 & 0,6583 & 0,6478 & 0,0105 & 0,7189 & 0,7223 & 0,0034 \\
\hline 291,56 & 0,6528 & 0,6446 & 0,0083 & 0,7126 & 0,7139 & 0,0013 \\
\hline 288,68 & 0,6494 & 0,6431 & 0,0063 & 0,7042 & 0,7036 & 0,0006 \\
\hline 285,79 & 0,6472 & 0,6426 & 0,0046 & 0,6947 & 0,6926 & 0,0021 \\
\hline 282,90 & 0,6455 & 0,6421 & 0,0034 & 0,6847 & 0,6816 & 0,0032 \\
\hline 280,01 & 0,6438 & 0,6412 & 0,0026 & 0,6747 & 0,6709 & 0,0039 \\
\hline 277,13 & 0,6418 & 0,6396 & 0,0022 & 0,6650 & 0,6609 & 0,0042 \\
\hline 274,24 & 0,6393 & 0,6372 & 0,0021 & 0,6558 & 0,6517 & 0,0041 \\
\hline 271,35 & 0,6362 & 0,6340 & 0,0022 & 0,6471 & 0,6432 & 0,0039 \\
\hline 268,47 & 0,6325 & 0,6300 & 0,0026 & 0,6390 & 0,6355 & 0,0034 \\
\hline 265,58 & 0,6283 & 0,6253 & 0,0030 & 0,6314 & 0,6285 & 0,0029 \\
\hline 262,69 & 0,6236 & 0,6201 & 0,0035 & 0,6242 & 0,6219 & 0,0023 \\
\hline 259,81 & 0,6184 & 0,6144 & 0,0040 & 0,6174 & 0,6156 & 0,0018 \\
\hline
\end{tabular}


Teoria

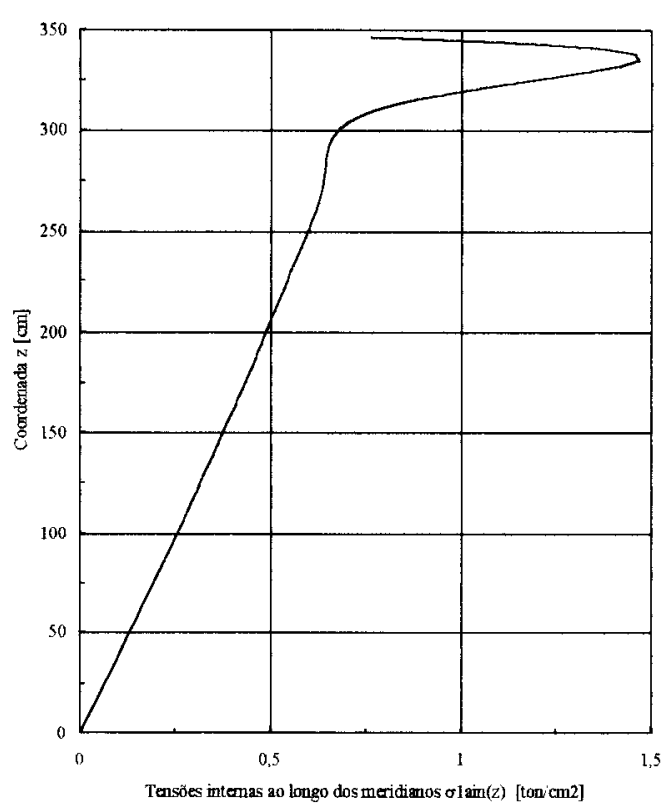

Elconentos Finitos

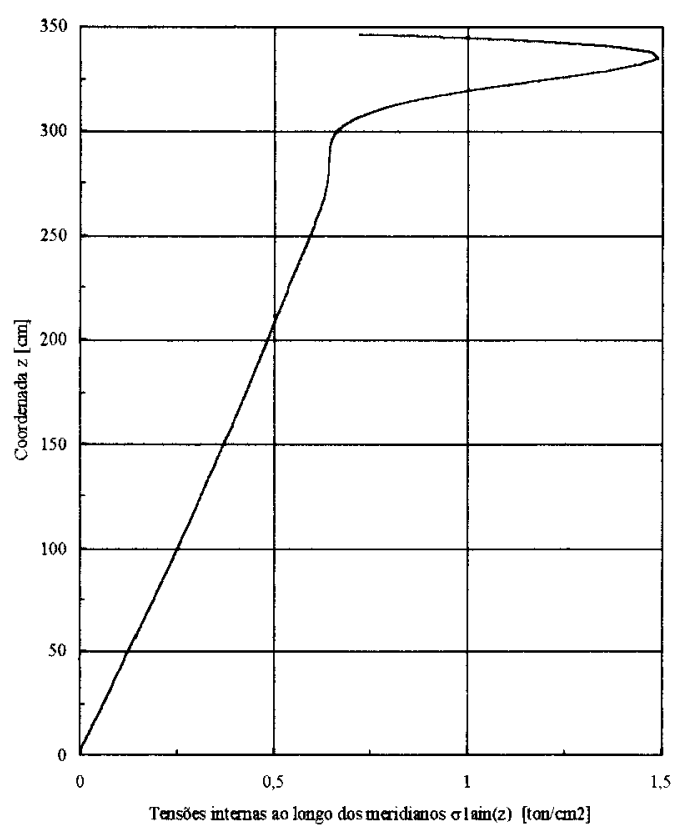

FIGURA 63 - Gráficos para os valores das tensões ao longo dos meridianos da superficie interna $\sigma$ lain(z) da casca cônica
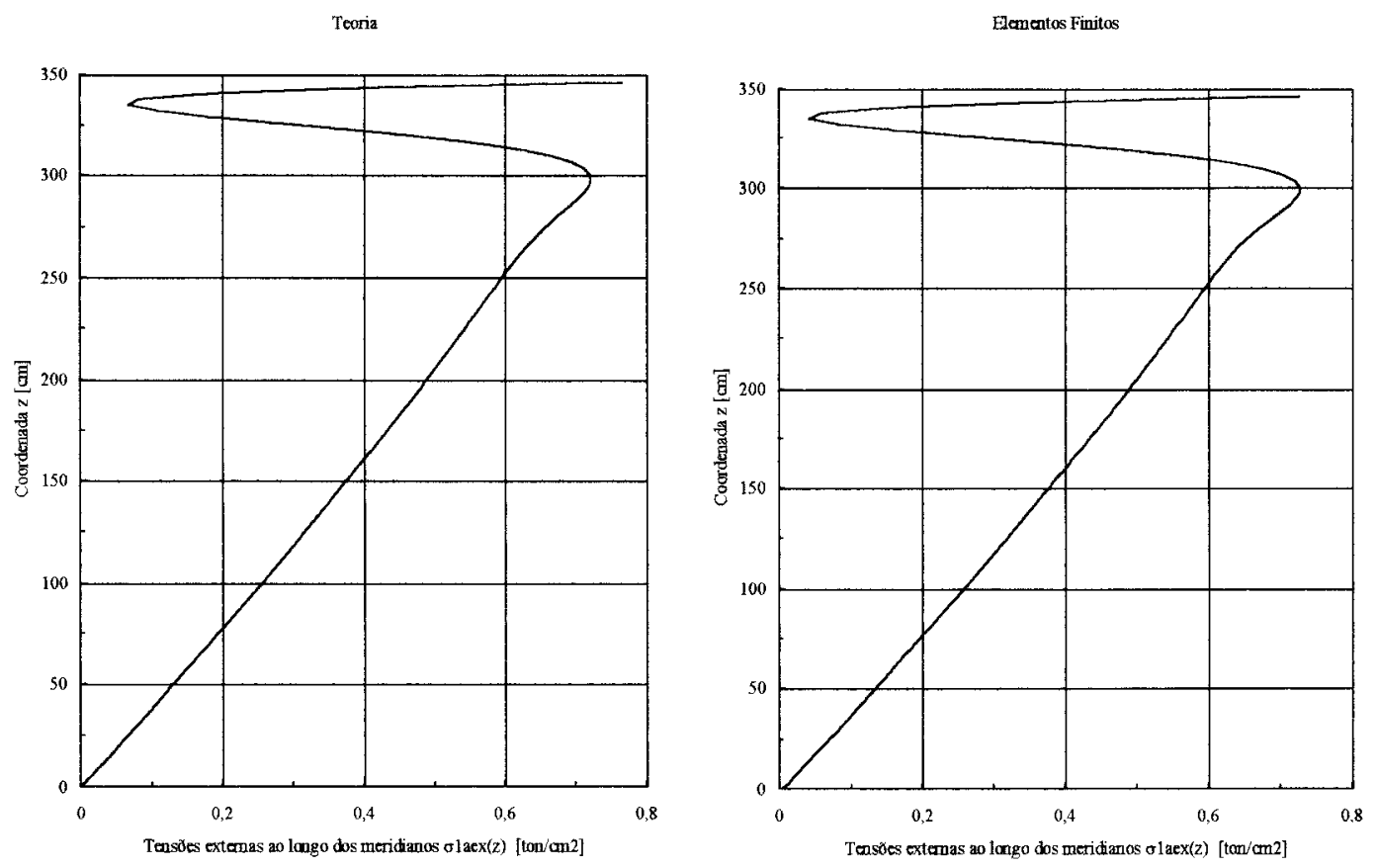

FIGURA 64 - Gráficos para os valores das tensões ao longo dos meridianos da superficie externa $\sigma$ laex(z) da casca cônica 
$\mathrm{E}$, para as tensões atuantes ao longo dos paralelos da superficie interna e externa da casca cônica, encontrou-se

TABELA 18 - Valores para as tensões ao longo dos paralelos da superfície interna $\sigma 2$ ain $(z)$ e externa $\sigma 2 a \operatorname{aex}(z)$ da casca

\begin{tabular}{|c|c|c|c|c|c|c|}
\hline \multirow[b]{2}{*}{$\begin{array}{c}\mathrm{z} \\
{[\mathrm{cm}]}\end{array}$} & \multicolumn{2}{|c|}{$\begin{array}{l}\sigma 2 \operatorname{ain}(\mathrm{z}) \\
{\left[\text { ton } / \mathrm{cm}^{2}\right]}\end{array}$} & \multirow[b]{2}{*}{ Diferença } & \multicolumn{2}{|c|}{$\begin{array}{l}\sigma 2 \mathrm{aex}(\mathrm{z}) \\
{\left[\mathrm{ton} / \mathrm{cm}^{2}\right]}\end{array}$} & \multirow[b]{2}{*}{ Diferença } \\
\hline & Teoria & $\begin{array}{c}\text { Elementos } \\
\text { Finitos } \\
\end{array}$ & & Teoria & \begin{tabular}{|c|} 
Elementos \\
Finitos \\
\end{tabular} & \\
\hline 346,41 & 0,2793 & 0,2529 & 0,0264 & 0,1943 & 0,1816 & 0,0127 \\
\hline 343,52 & 0,6357 & 0,6175 & 0,0182 & 0,3292 & 0,3179 & 0,0113 \\
\hline 340,64 & 0,9239 & 0,9070 & 0,0168 & 0,4957 & 0,4798 & 0,0159 \\
\hline 337,75 & 1,1455 & 1,1327 & 0,0128 & 0,6698 & 0,6530 & 0,0168 \\
\hline 334,86 & 1,3067 & 1,2987 & 0,0080 & 0,8353 & 0,8205 & 0,0148 \\
\hline 331,98 & 1,4159 & 1,4123 & 0,0037 & 0,9823 & 0,9712 & 0,0110 \\
\hline 329,09 & 1,4826 & 1,4819 & 0,0007 & 1,1056 & 1,0991 & 0,0065 \\
\hline 326,20 & 1,5158 & 1,5164 & 0,0006 & 1,2037 & 1,2014 & 0,0023 \\
\hline 323,32 & 1,5241 & 1,5246 & 0,0004 & 1,2774 & 1,2786 & 0,0012 \\
\hline 320,43 & 1,5150 & 1,5140 & 0,0010 & 1,3291 & 1,3327 & 0,0035 \\
\hline 317,54 & 1,4944 & 1,4912 & 0,0032 & 1,3620 & 1,3665 & 0,0045 \\
\hline 314,66 & 1,4671 & 1,4612 & 0,0059 & 1,3794 & 1,3839 & 0,0045 \\
\hline 311,77 & 1,4369 & 1,4283 & 0,0086 & 1,3847 & 1,3883 & 0,0036 \\
\hline 308,88 & 1,4063 & 1,3952 & 0,0111 & 1,3812 & 1,3832 & 0,0020 \\
\hline 306,00 & 1,3770 & 1,3640 & 0,0131 & 1,3713 & 1,3714 & 0,0000 \\
\hline 303,11 & 1,3500 & 1,3355 & 0,0145 & 1,3574 & 1,3554 & 0,0020 \\
\hline 300,22 & 1,3258 & 1,3106 & 0,0152 & 1,3412 & 1,3373 & 0,0039 \\
\hline 297,34 & 1,3045 & 1,2890 & 0,0155 & 1,3240 & 1,3185 & 0,0055 \\
\hline 294,45 & 1,2859 & 1,2707 & 0,0152 & 1,3066 & 1,3000 & 0,0066 \\
\hline 291,56 & 1,2697 & 1,2551 & 0,0147 & 1,2897 & 1,2823 & 0,0074 \\
\hline 288,68 & 1,2556 & 1,2418 & 0,0138 & 1,2736 & 1,2659 & 0,0078 \\
\hline 285,79 & 1,2432 & 1,2303 & 0,0129 & 1,2585 & 1,2508 & 0,0078 \\
\hline 282,90 & 1,2320 & 1,2200 & 0,0120 & 1,2445 & 1,2370 & 0,0075 \\
\hline 280,01 & 1,2217 & 1,2107 & 0,0110 & 1,2314 & 1,2244 & 0,0070 \\
\hline 277,13 & 1,2120 & 1,2017 & 0,0103 & 1,2192 & 1,2128 & 0,0064 \\
\hline 274,24 & 1,2026 & 1,1930 & 0,0096 & 1,2077 & 1,2019 & 0,0058 \\
\hline 271,35 & 1,1935 & 1,1843 & 0,0092 & 1,1967 & 1,1916 & 0,0052 \\
\hline 268,47 & 1,1843 & 1,1755 & 0,0089 & 1,1861 & 1,1817 & 0,0045 \\
\hline 265,58 & 1,1751 & 1,1664 & 0,0088 & 1,1759 & 1,1719 & 0,0040 \\
\hline 262,69 & 1,1658 & 1,1571 & 0,0087 & 1,1658 & 1,1623 & 0,0035 \\
\hline 259,81 & 1,1562 & 1,1476 & 0,0087 & 1,1558 & 1,1526 & 0,0032 \\
\hline
\end{tabular}



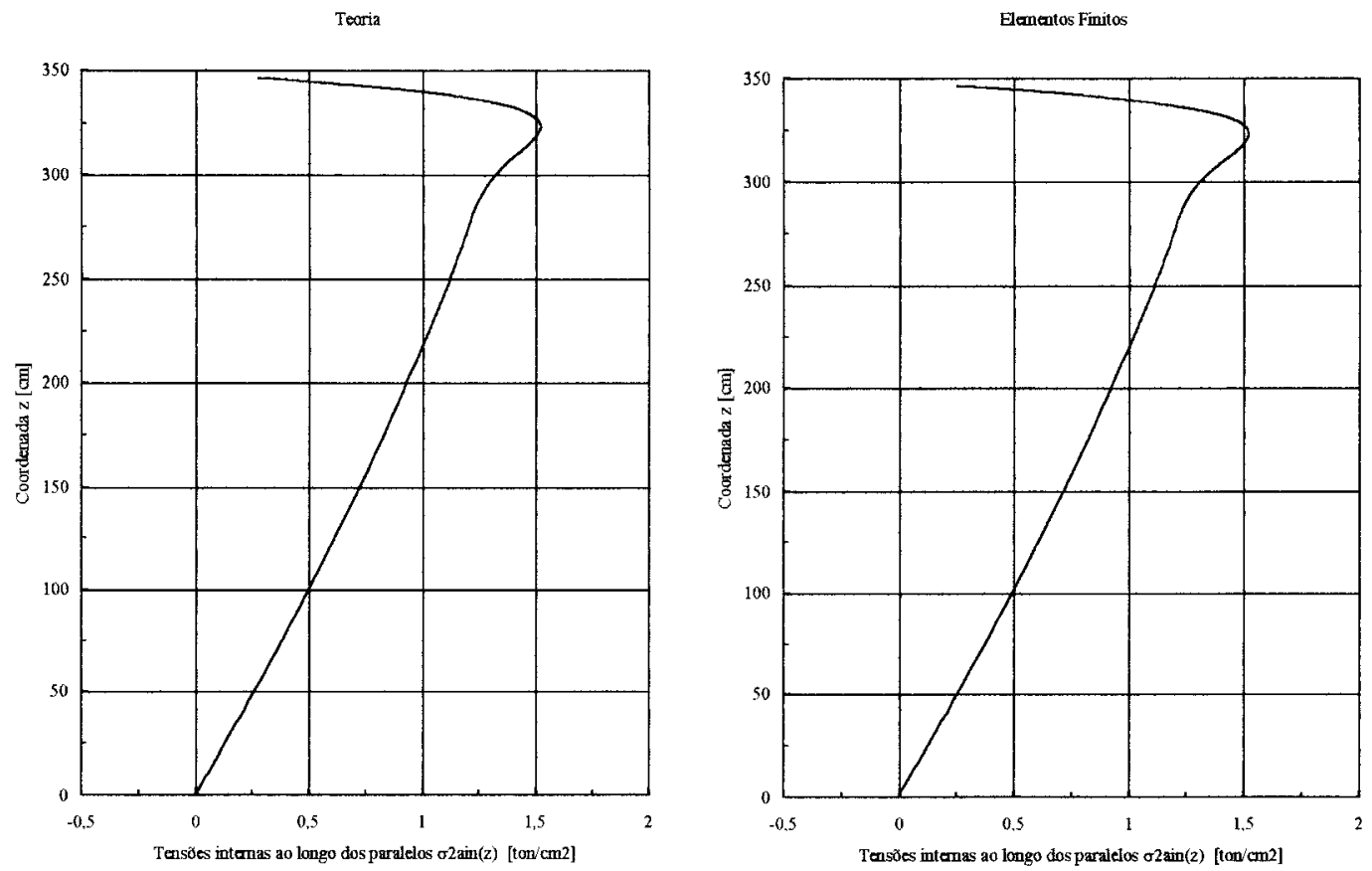

FIGURA 65 - Gráficos para os valores das tensões ao longo dos paralelos da superficie interna $\sigma 2 a i n(z)$ da casca cônica
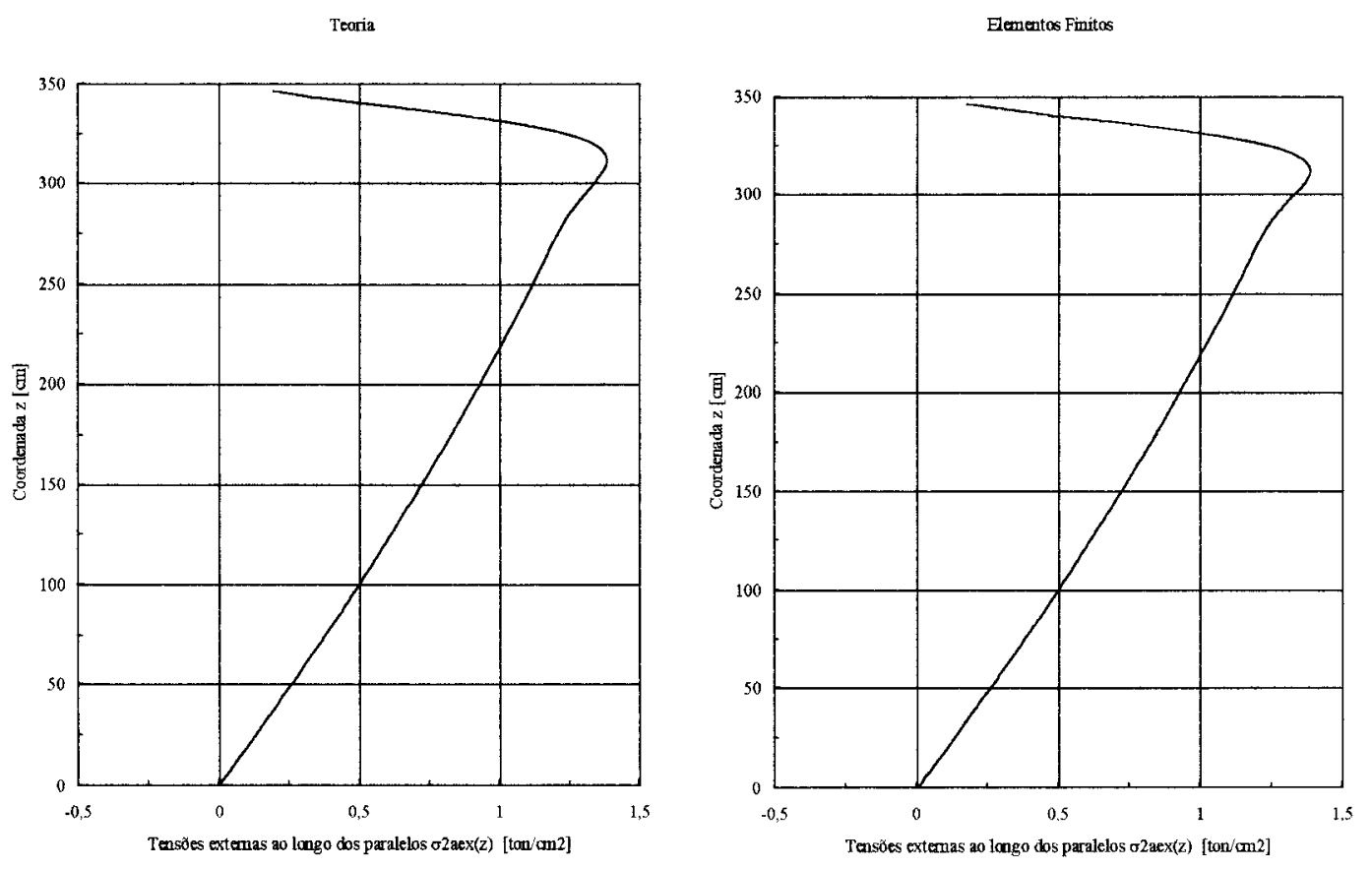

FIGURA 66 - Gráficos para os valores das tensões ao longo dos paralelos da superfície externa $\sigma 2 \mathrm{aex}(\mathrm{z})$ da casca cônica 
Observar, nos gráficos abaixo, que as tensões teóricas ao longo dos meridianos e paralelos da casca cônica, como para borda engastada, possuem seus valores praticamente coincidêntes com as tensões de membrana para a região abaixo de $z=H-\lambda z=261,18[\mathrm{~cm}]$.

Teoria

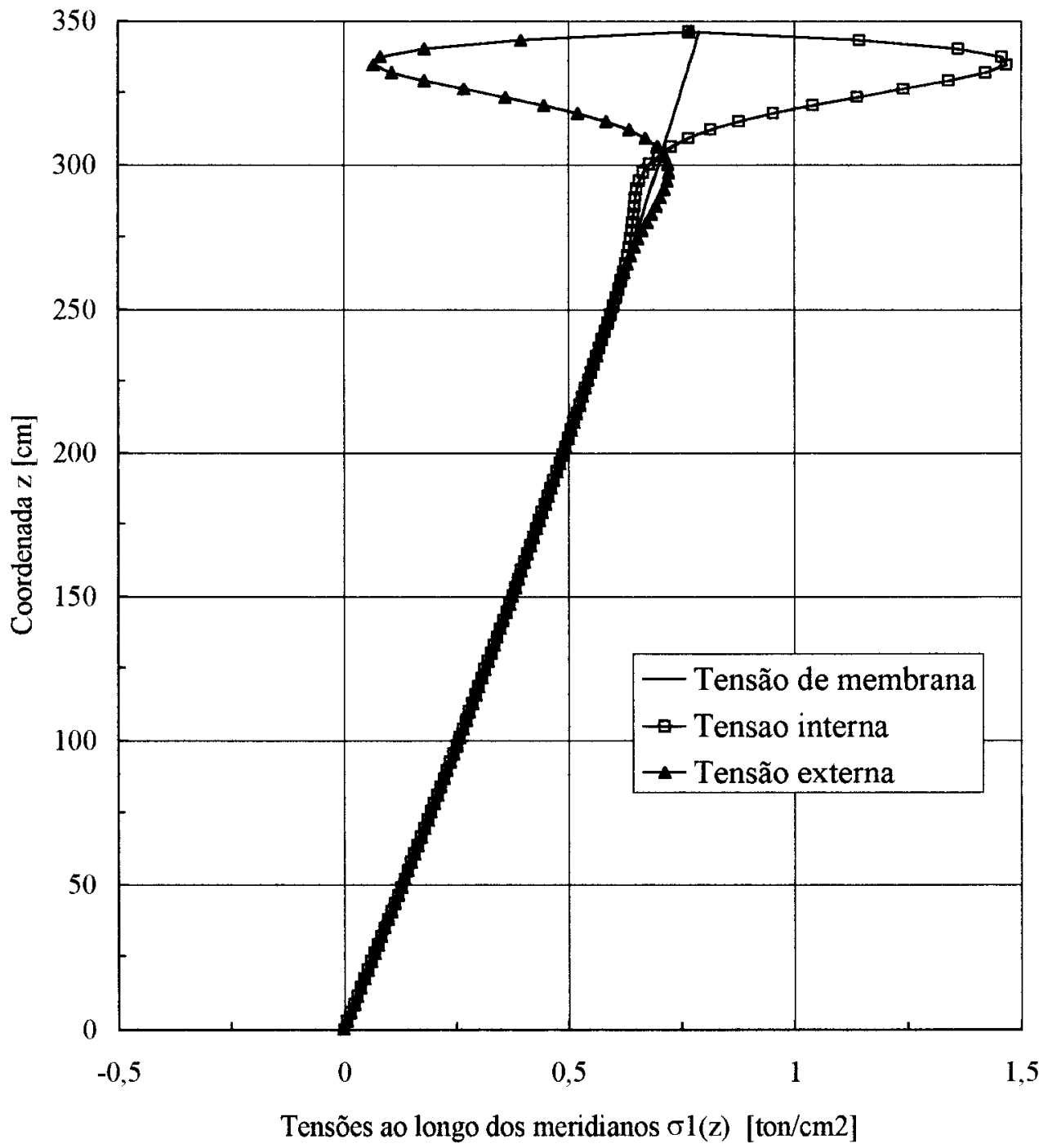

FIGURA 67 - Gráfico comparativo para os valores das tensões ao longo dos meridianos da casca cônica : $\sigma \operatorname{lm}(\mathrm{z}), \sigma \operatorname{lain}(\mathrm{z})$ e $\sigma \operatorname{laex}(\mathrm{z})$ 
Teoria

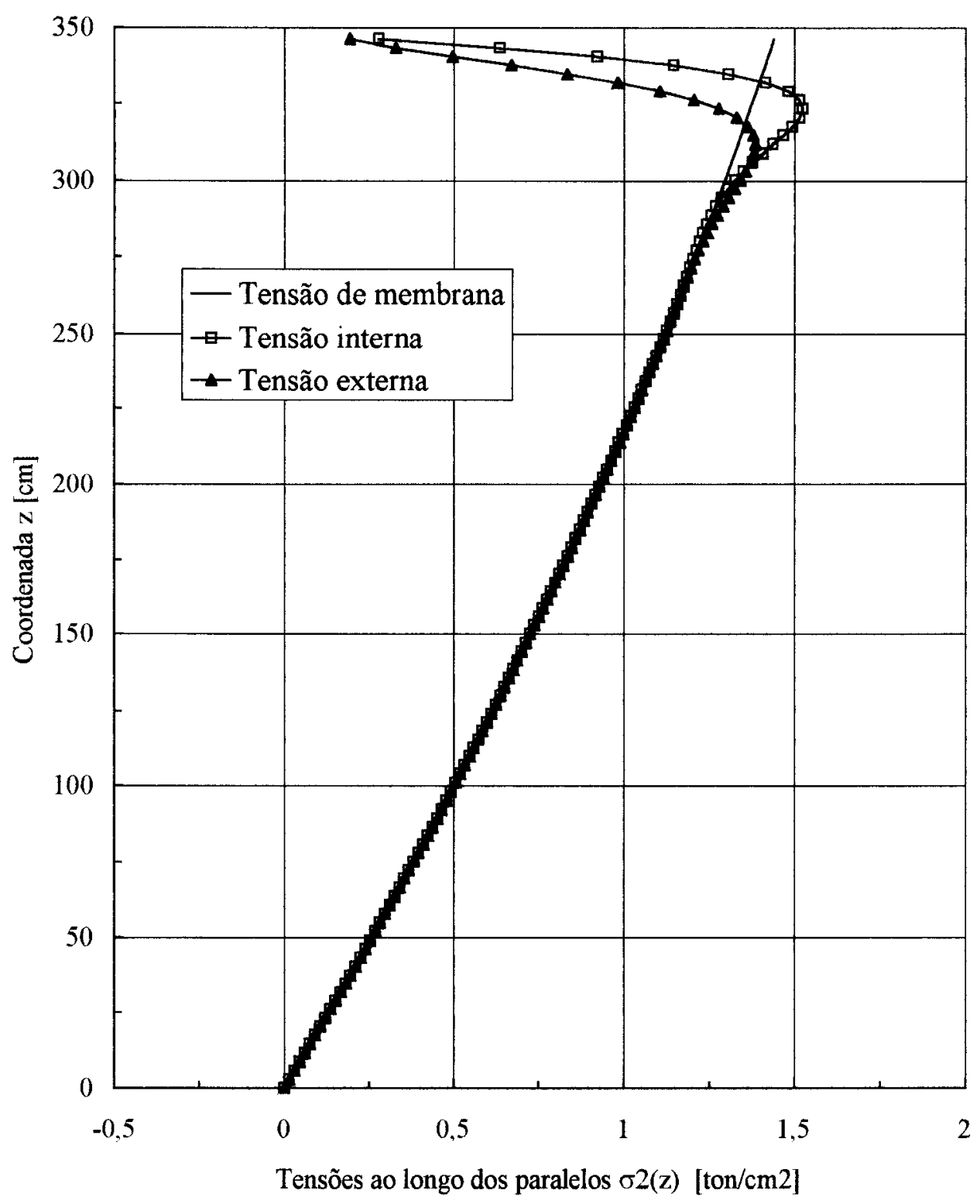

FIGURA 68 - Gráfico comparativo para os valores das tensões ao longo dos paralelos da casca cônica : $\sigma 2 \mathrm{~m}(\mathrm{z}), \sigma 2 \mathrm{ain}(\mathrm{z})$ e $\sigma 2 \mathrm{aex}(\mathrm{z})$ 


\subsection{CALCULANDO E OBTENDO RESULTADOS PARA A CASCA CÔNICA INCLINAÇÃO $\theta \mathrm{r}=4^{\circ}$ COM A HORIZONTAL}

Através das equações teóricas do capítulo 2 para casca cônica, e com os dados abaixo :

\begin{tabular}{|c|c|c|c|c|c|c|}
\hline $\begin{array}{c}\mathrm{E} \\
{\left[\text { ton } / \mathrm{cm}^{2}\right]}\end{array}$ & $\begin{array}{c}v \\
(\text { Aço) }\end{array}$ & $\begin{array}{c}\gamma \\
{\left[\text { ton } / \mathrm{cm}^{3}\right]}\end{array}$ & $\begin{array}{c}\theta \mathrm{r} \\
{[\text { graus] }}\end{array}$ & $\begin{array}{c}\alpha \mathrm{r} \\
\text { [graus] }]\end{array}$ & $\begin{array}{c}\mathrm{D} \\
{[\mathrm{cm}]}\end{array}$ & $\begin{array}{c}\mathrm{r} \\
{[\mathrm{cm}]}\end{array}$ \\
\hline 2100 & 0,3 & $1,00 \mathrm{E}-06$ & 45 & 45 & 1200 & 600 \\
\hline
\end{tabular}

\begin{tabular}{|c|c|c|c|}
\hline $\begin{array}{c}\mathrm{R} \\
{[\mathrm{cm}]}\end{array}$ & $\begin{array}{c}\mathrm{H} \\
{[\mathrm{cm}]}\end{array}$ & $\begin{array}{c}\mathrm{h} \\
{[\mathrm{cm}]}\end{array}$ & $\begin{array}{c}\mathrm{t} \\
{[\mathrm{cm}]}\end{array}$ \\
\hline 848,53 & 600 & 1200 & 1 \\
\hline
\end{tabular}

Encontram-se :

\begin{tabular}{|c|c|c|c|c|c|c|}
\hline $\begin{array}{c}\beta \\
{\left[\text { ton } / \mathrm{cm}^{3}\right]}\end{array}$ & $\begin{array}{c}\mathrm{B} \\
{[\text { ton } / \mathrm{cm}]}\end{array}$ & $\begin{array}{c}\alpha \\
{\left[\mathrm{cm}^{-1}\right]}\end{array}$ & $\begin{array}{c}\xi \mathrm{h} \\
{\left[\mathrm{cm}^{2} / \text { ton }\right]}\end{array}$ & $\begin{array}{c}\xi \mathrm{m} \\
{[\mathrm{cm} / \text { ton }]}\end{array}$ & $\begin{array}{c}\phi \mathrm{h} \\
{[\mathrm{cm} / \text { ton }]}\end{array}$ & $\begin{array}{c}\phi \mathrm{m} \\
{\left[\text { ton }^{-1}\right]}\end{array}$ \\
\hline 0,0029 & 192,3077 & 0,0441 & 15,1294 & 0,9442 & 0,9442 & 0,1178 \\
\hline
\end{tabular}

\begin{tabular}{|c|c|c|}
\hline $\begin{array}{c}\text { Mce } \\
{[\text { ton } . \mathrm{cm} / \mathrm{cm}]}\end{array}$ & $\begin{array}{c}\text { Hce } \\
{[\text { ton } / \mathrm{cm}]}\end{array}$ & $\begin{array}{c}\text { Hca } \\
{[\text { ton } / \mathrm{cm}]}\end{array}$ \\
\hline 0,2467 & $-0,0313$ & $-0,0159$ \\
\hline
\end{tabular}

E para o comprimento ao longo do meridiano da casca necessário para o amortecimento das tensões de flexão causadas pela "perturbação de borda" medido a partir do vínculo :

$$
-\lambda_{\text {cone }}=143,74[\mathrm{~cm}]
$$


$\mathrm{Na}$ vertical chamou-se este valor de $\lambda z$ que é igual a $\lambda_{\text {cone. }} \operatorname{sen} \theta \mathrm{r}=101,64$ [cm], e portanto a tabela de valores para a casca cônica deveria ser apresentada das coordenadas " $\mathrm{z}$ " igual a $\mathrm{H}-\lambda z=498,36$ até $H=600$. Adotou-se de 495 até 600 $[\mathrm{cm}]$.

\subsubsection{CASCA CÔNICA COM BORDA SUPERIOR ENGASTADA}

Para os deslocamentos radiais e rotações ao longo do meridiano da superficie média da casca cônica encontrou-se

TABELA 19 - Valores dos deslocamentos radiais $\xi \mathrm{e}(\mathrm{z})$ e rotações $\phi \mathrm{e}(\mathrm{z})$

\begin{tabular}{|c|c|c|c|}
\hline & \multicolumn{2}{|c|}{$\begin{array}{l}\xi \mathrm{e}(\mathrm{z}) \\
{[\mathrm{cm}]}\end{array}$} & \\
\hline $\begin{array}{c}\mathrm{z} \\
{[\mathrm{cm}]}\end{array}$ & Teoria & \begin{tabular}{c|} 
Elementos \\
Finitos \\
\end{tabular} & Diferença \\
\hline 600,00 & 0,0000 & 0,0000 & 0,0000 \\
\hline 595,00 & 0,0176 & 0,0173 & 0,0003 \\
\hline 590,00 & 0,0569 & 0,0568 & 0,0001 \\
\hline 585,00 & 0,1019 & 0,1024 & 0,0005 \\
\hline 580,00 & 0,1431 & 0,1441 & 0,0010 \\
\hline 575,00 & 0,1760 & 0,1773 & 0,0013 \\
\hline 570,00 & 0,1993 & 0,2006 & 0,0013 \\
\hline 565,00 & 0,2137 & 0,2147 & 0,0009 \\
\hline 560,00 & 0,2209 & 0,2213 & 0,0004 \\
\hline 555,00 & 0,2228 & 0,2226 & 0,0002 \\
\hline 550,00 & 0,2212 & 0,2204 & 0,0008 \\
\hline 545,00 & 0,2175 & 0,2162 & 0,0013 \\
\hline 540,00 & 0,2128 & 0,2112 & 0,0016 \\
\hline 535,00 & 0,2078 & 0,2060 & 0,0018 \\
\hline 530,00 & 0,2030 & 0,2012 & 0,0018 \\
\hline 525,00 & 0,1985 & 0,1968 & 0,0017 \\
\hline 520,00 & 0,1945 & 0,1929 & 0,0015 \\
\hline 515,00 & 0,1908 & 0,1894 & 0,0013 \\
\hline 510,00 & 0,1874 & 0,1863 & 0,0012 \\
\hline 505,00 & 0,1843 & 0,1833 & 0,0010 \\
\hline 500,00 & 0,1814 & 0,1805 & 0,0009 \\
\hline 495,00 & 0,1785 & 0,1777 & 0,0008 \\
\hline
\end{tabular}

\begin{tabular}{|c|c|c|}
\hline \multicolumn{2}{|c|}{$\begin{array}{c}\phi \mathrm{e}(\mathrm{z}) \\
\text { [radianos] }\end{array}$} & \multirow[b]{2}{*}{ Diferença } \\
\hline Teoria & $\begin{array}{c}\text { Elementos } \\
\text { Finitos } \\
\end{array}$ & \\
\hline 0,0000 & 0,0000 & 0,0000 \\
\hline$-0,0065$ & $-0,0066$ & 0,0001 \\
\hline$-0,0090$ & $-0,0091$ & 0,0001 \\
\hline$-0,0090$ & $-0,0091$ & 0,0002 \\
\hline$-0,0076$ & $-0,0077$ & 0,0001 \\
\hline$-0,0057$ & $-0,0058$ & 0,0000 \\
\hline$-0,0039$ & $-0,0038$ & 0,0000 \\
\hline$-0,0022$ & $-0,0021$ & 0,0001 \\
\hline$-0,0010$ & $-0,0009$ & 0,0001 \\
\hline$-0,0001$ & 0,0000 & 0,0001 \\
\hline 0,0004 & 0,0006 & 0,0001 \\
\hline 0,0007 & 0,0008 & 0,0001 \\
\hline 0,0009 & 0,0009 & 0,0000 \\
\hline 0,0009 & 0,0009 & 0,0000 \\
\hline 0,0008 & 0,0008 & 0,0000 \\
\hline 0,0007 & 0,0007 & 0,0000 \\
\hline 0,0007 & 0,0006 & 0,0000 \\
\hline 0,0006 & 0,0005 & 0,0000 \\
\hline 0,0005 & 0,0005 & 0,0000 \\
\hline 0,0005 & 0,0005 & 0,0000 \\
\hline 0,0005 & 0,0004 & 0,0000 \\
\hline 0,0004 & 0,0004 & 0,0000 \\
\hline
\end{tabular}


Teoria

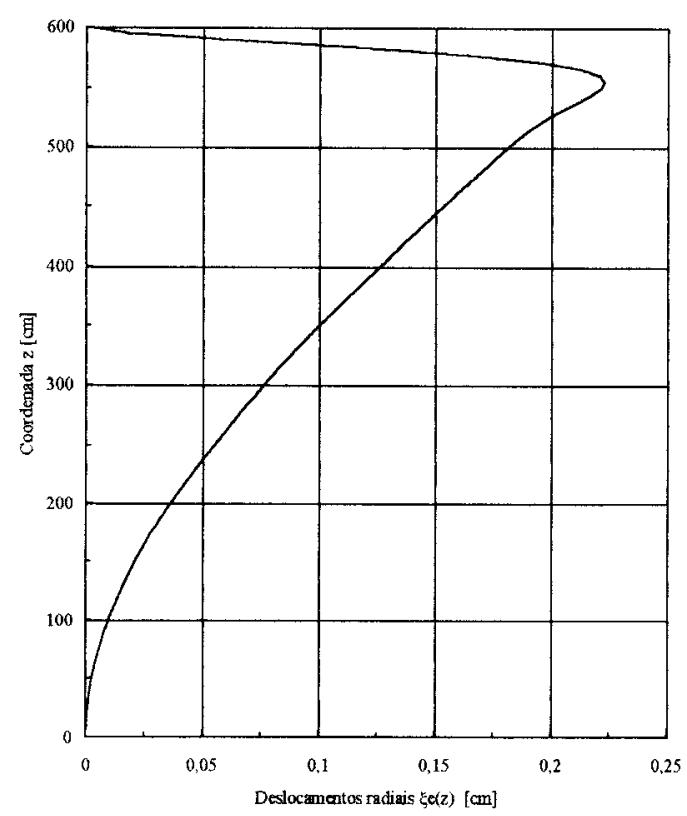

Elementos Finitos

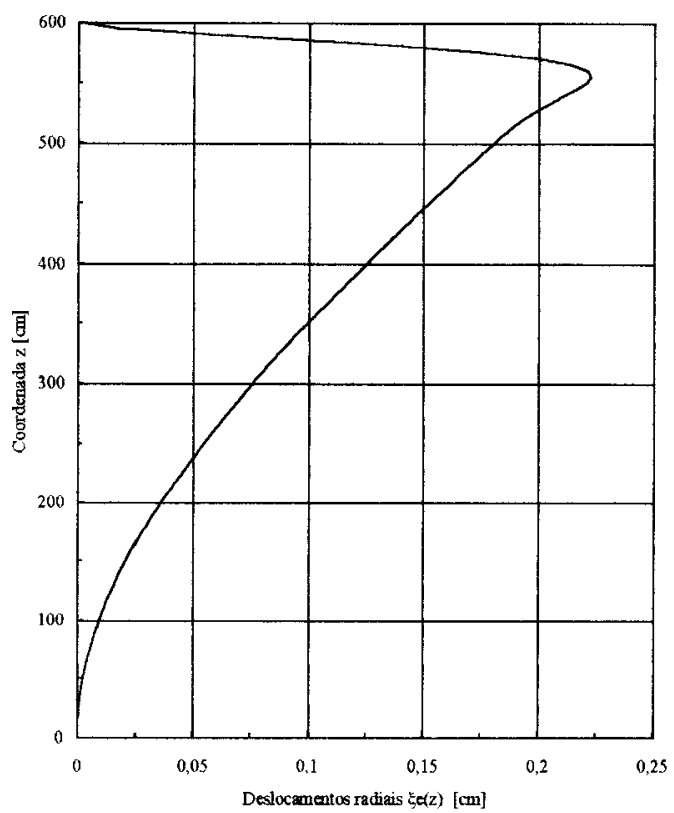

FIGURA 69 - Gráficos para os valores dos deslocamentos radiais $\xi \mathrm{e}(\mathrm{z})$ ao longo do meridiano da superficie média da casca cônica

Tearia

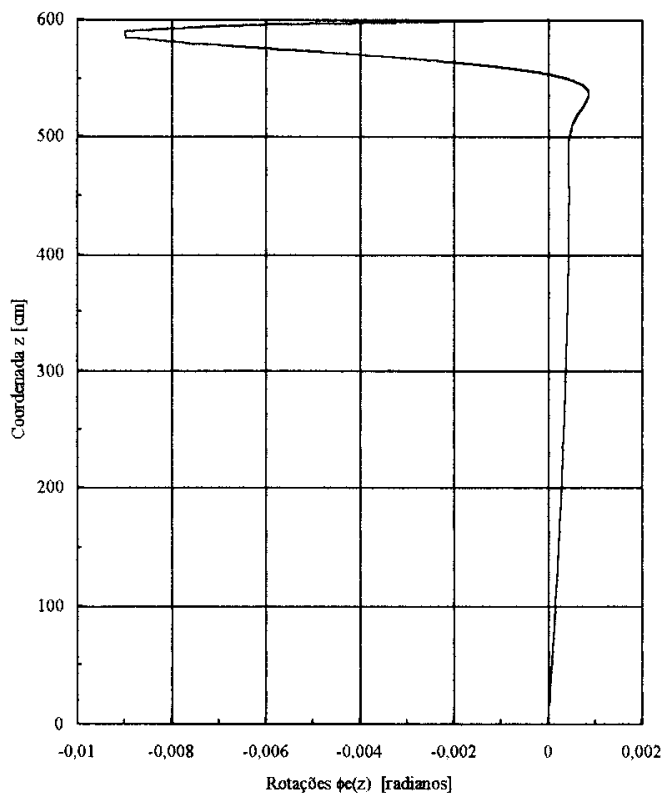

Elementos Finitos

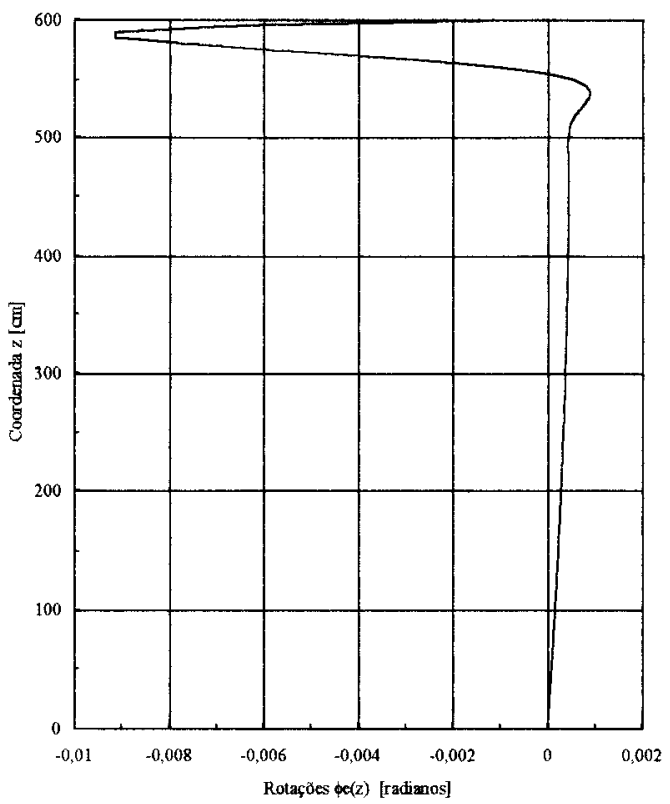

FIGURA 70 - Gráficos para os valores das rotações $\phi \mathrm{e}(\mathrm{z})$ ao longo do meridiano da superficie média da casca cônica 
Lembrando que a coluna "Diferença", da tabela, apresenta os valores das diferenças absolutas entre os parâmetros obtidos pelas equações da teoria aproximada e os obtidos pelo cálculo através do método dos elementos finitos.

Os momentos solicitantes ao longo dos paralelos e dos meridianos da superficie média da casca cônica e suas correspondentes diferenças absolutas foram :

TABELA 20 - Valores dos momentos ao longo dos paralelos $\mathrm{Mle}(\mathrm{z})$ e dos meridianos M2e(z)

\begin{tabular}{|c|r|r|r|}
\cline { 2 - 3 } \multicolumn{1}{c|}{} & \multicolumn{2}{c|}{$\begin{array}{c}\text { M1 e(z) } \\
\text { [ton.cm/cm] }\end{array}$} & \multicolumn{1}{c}{} \\
\hline $\begin{array}{c}z \\
{[\mathrm{~cm}]}\end{array}$ & Teoria & $\begin{array}{c}\text { Elementos } \\
\text { Finitos }\end{array}$ & Diferença \\
\hline 600,00 & 0,2467 & 0,2440 & 0,0027 \\
\hline 595,00 & 0,1147 & 0,1126 & 0,0021 \\
\hline 590,00 & 0,0277 & 0,0250 & 0,0027 \\
\hline 585,00 & $-0,0229$ & $-0,0264$ & 0,0035 \\
\hline 580,00 & $-0,0468$ & $-0,0505$ & $\mathbf{0 , 0 0 3 8}$ \\
\hline 575,00 & $-0,0529$ & $-0,0563$ & 0,0034 \\
\hline 570,00 & $-0,0486$ & $-0,0512$ & 0,0026 \\
\hline 565,00 & $-0,0394$ & $-0,0409$ & 0,0015 \\
\hline 560,00 & $-0,0288$ & $-0,0293$ & 0,0004 \\
\hline 555,00 & $-0,0191$ & $-0,0187$ & 0,0004 \\
\hline 550,00 & $-0,0111$ & $-0,0102$ & 0,0009 \\
\hline 545,00 & $-0,0053$ & $-0,0041$ & 0,0012 \\
\hline 540,00 & $-0,0014$ & $-0,0002$ & 0,0012 \\
\hline 535,00 & 0,0009 & 0,0019 & 0,0010 \\
\hline 530,00 & 0,0020 & 0,0027 & 0,0007 \\
\hline 525,00 & 0,0023 & 0,0027 & 0,0004 \\
\hline 520,00 & 0,0021 & 0,0023 & 0,0002 \\
\hline 515,00 & 0,0017 & 0,0017 & 0,0000 \\
\hline 510,00 & 0,0013 & 0,0011 & 0,0001 \\
\hline 505,00 & 0,0009 & 0,0007 & 0,0002 \\
\hline 500,00 & 0,0005 & 0,0003 & 0,0002 \\
\hline 495,00 & 0,0002 & 0,0001 & 0,0001 \\
\hline
\end{tabular}

\begin{tabular}{|r|r|r|}
\hline \multicolumn{2}{|c|}{$\begin{array}{r}\text { M2e(z) } \\
\text { [ton.cm/cm] }\end{array}$} & \\
\cline { 1 - 2 } Teoria & $\begin{array}{c}\text { Elementos } \\
\text { Finitos }\end{array}$ & Diferença \\
\hline 0,0739 & 0,0732 & 0,0007 \\
\hline 0,0328 & 0,0324 & 0,0004 \\
\hline 0,0062 & 0,0056 & 0,0006 \\
\hline$-0,0090$ & $-0,0098$ & 0,0008 \\
\hline$-0,0159$ & $-0,0168$ & $\mathbf{0 , 0 0 0 9}$ \\
\hline$-0,0173$ & $-0,0181$ & 0,0009 \\
\hline$-0,0156$ & $-0,0162$ & 0,0006 \\
\hline$-0,0124$ & $-0,0127$ & 0,0003 \\
\hline$-0,0090$ & $-0,0090$ & 0,0000 \\
\hline$-0,0058$ & $-0,0056$ & 0,0003 \\
\hline$-0,0033$ & $-0,0029$ & 0,0004 \\
\hline$-0,0015$ & $-0,0010$ & 0,0005 \\
\hline$-0,0003$ & 0,0001 & 0,0005 \\
\hline 0,0004 & 0,0008 & 0,0004 \\
\hline 0,0007 & 0,0010 & 0,0003 \\
\hline 0,0007 & 0,0010 & 0,0002 \\
\hline 0,0007 & 0,0008 & 0,0002 \\
\hline 0,0005 & 0,0006 & 0,0001 \\
\hline 0,0004 & 0,0005 & 0,0001 \\
\hline 0,0003 & 0,0003 & 0,0001 \\
\hline 0,0002 & 0,0002 & 0,0001 \\
\hline 0,0001 & 0,0001 & 0,0001 \\
\hline & & \\
\hline
\end{tabular}


Teoria

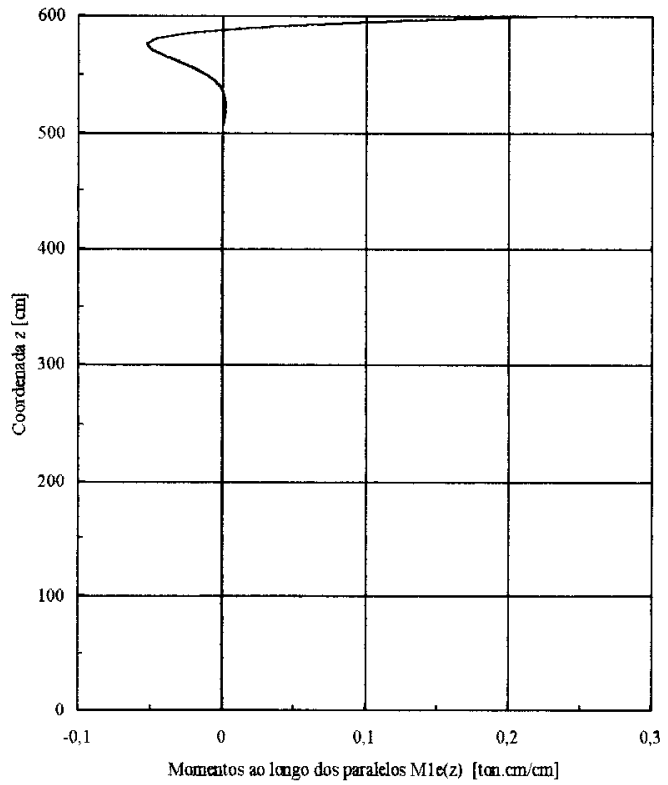

Elementos Finitos

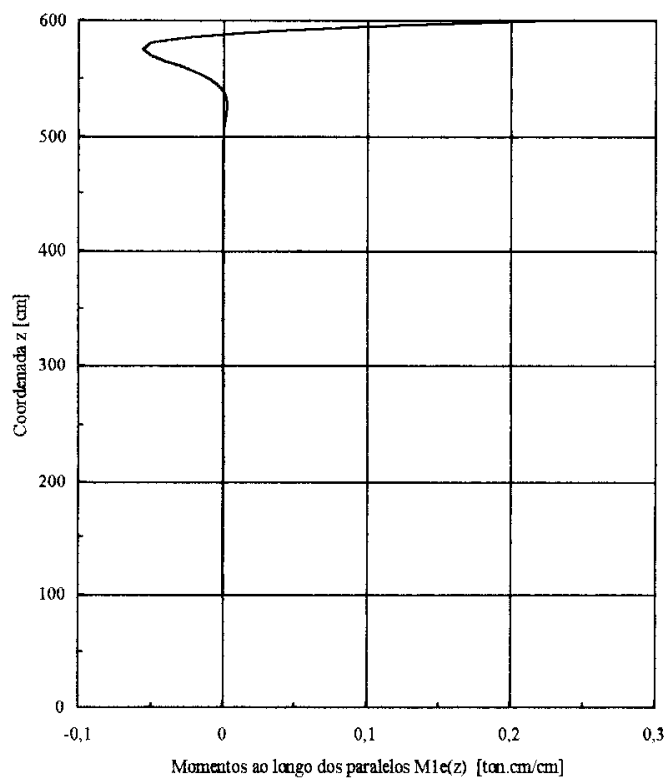

FIGURA 71 - Gráficos para os valores dos momentos ao longo dos paralelos Mle(z) da superficie média da casca cônica

Teoria

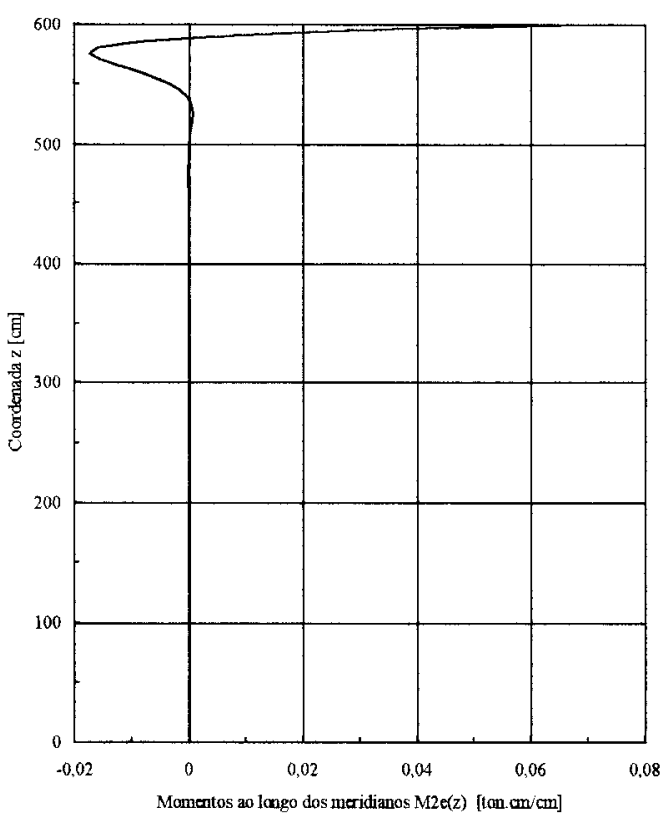

Elementos Finitos

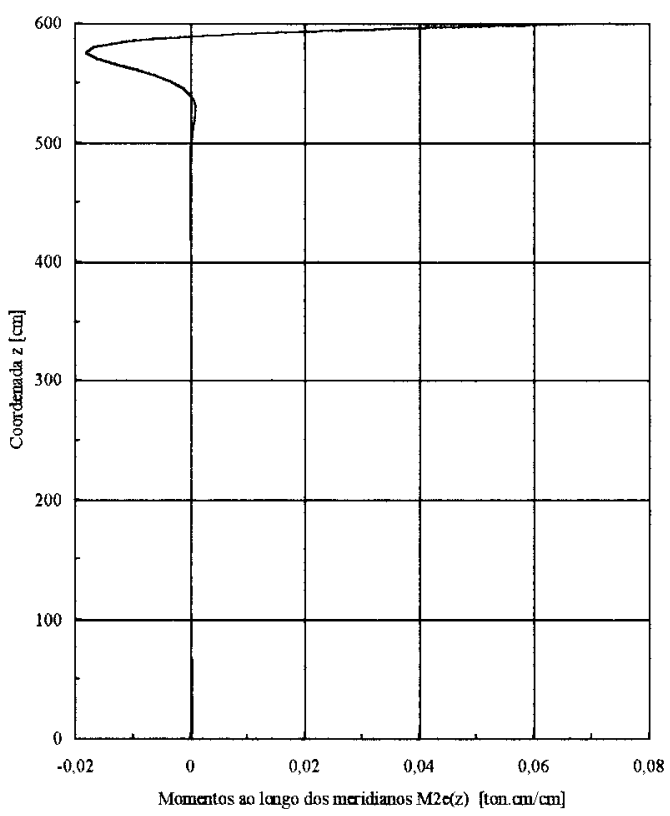

FIGURA 72 - Gráficos para os valores dos momentos ao longo dos meridianos M2e(z) da superficie média da casca cônica 
Para as tensões atuantes ao longo dos meridianos da superficie interna e externa da casca cônica obteve-se

TABELA 21 - Valores para as tensões ao longo dos meridianos da superficie interna $\sigma$ lein(z) e externa $\sigma$ leex(z) da casca

\begin{tabular}{|c|c|c|c|}
\hline & \multicolumn{2}{|c|}{$\begin{array}{c}\sigma l e i n(z) \\
{\left[\operatorname{ton} / \mathrm{cm}^{2}\right]}\end{array}$} & \multirow[b]{2}{*}{ Diferença } \\
\hline $\begin{array}{c}\mathrm{z} \\
{[\mathrm{cm}]}\end{array}$ & Teoria & $\begin{array}{c}\text { Elementos } \\
\text { Finitos }\end{array}$ & \\
\hline 600,00 & $-0,9081$ & $-0,9031$ & 0,0050 \\
\hline 595,00 & $-0,1132$ & $-0,1102$ & 0,0029 \\
\hline 590,00 & 0,4114 & 0,4226 & 0,0113 \\
\hline 585,00 & 0,7157 & 0,7346 & 0,0189 \\
\hline 580,00 & 0,8582 & 0,8806 & 0,0223 \\
\hline 575,00 & 0,8932 & 0,9140 & 0,0209 \\
\hline 570,00 & 0,8649 & 0,8807 & 0,0158 \\
\hline 565,00 & 0,8064 & 0,8153 & 0,0089 \\
\hline 560,00 & 0,7394 & 0,7414 & 0,0020 \\
\hline 555,00 & 0,6769 & 0,6733 & 0,0036 \\
\hline 550,00 & 0,6252 & 0,6178 & 0,0074 \\
\hline 545,00 & 0,5860 & 0,5769 & 0,0091 \\
\hline 540,00 & 0,5587 & 0,5494 & 0,0093 \\
\hline 535,00 & 0,5409 & 0,5327 & 0,0083 \\
\hline 530,00 & 0,5304 & 0,5237 & 0,0067 \\
\hline 525,00 & 0,5246 & 0,5196 & 0,0050 \\
\hline 520,00 & 0,5216 & 0,5182 & 0,0035 \\
\hline 515,00 & 0,5200 & 0,5177 & 0,0023 \\
\hline 510,00 & 0,5188 & 0,5172 & 0,0015 \\
\hline 505,00 & 0,5174 & 0,5162 & 0,0012 \\
\hline 500,00 & 0,5155 & 0,5144 & 0,0011 \\
\hline 495,00 & 0,5130 & 0,5117 & 0,0013 \\
\hline
\end{tabular}

\begin{tabular}{|c|c|c|}
\hline \multicolumn{2}{|c|}{$\begin{array}{l}\sigma l \operatorname{eex}(\mathrm{z}) \\
{\left[\mathrm{ton} / \mathrm{cm}^{2}\right]}\end{array}$} & \multirow[b]{2}{*}{ Diferença } \\
\hline Teoria & $\begin{array}{c}\text { Elementos } \\
\text { Finitos } \\
\end{array}$ & \\
\hline 2,0519 & 2,0246 & 0,0273 \\
\hline 1,2634 & 1,2414 & 0,0220 \\
\hline 0,7433 & 0,7223 & 0,0210 \\
\hline 0,4407 & 0,4180 & 0,0227 \\
\hline 0,2972 & 0,2743 & 0,0229 \\
\hline 0,2589 & 0,2387 & 0,0202 \\
\hline 0,2818 & 0,2667 & 0,0151 \\
\hline 0,3338 & 0,3248 & 0,0091 \\
\hline 0,3934 & 0,3901 & 0,0033 \\
\hline 0,4480 & 0,4492 & 0,0012 \\
\hline 0,4917 & 0,4957 & 0,0040 \\
\hline 0,5226 & 0,5277 & 0,0051 \\
\hline 0,5419 & 0,5466 & 0,0047 \\
\hline 0,5515 & 0,5550 & 0,0035 \\
\hline 0,5541 & 0,5559 & 0,0018 \\
\hline 0,5520 & 0,5521 & 0,0001 \\
\hline 0,5471 & 0,5457 & 0,0014 \\
\hline 0,5408 & 0,5383 & 0,0024 \\
\hline 0,5341 & 0,5310 & 0,0031 \\
\hline 0,5276 & 0,5243 & 0,0033 \\
\hline 0,5215 & 0,5182 & 0,0033 \\
\hline 0,5160 & 0,5129 & 0,0031 \\
\hline
\end{tabular}


Teoria

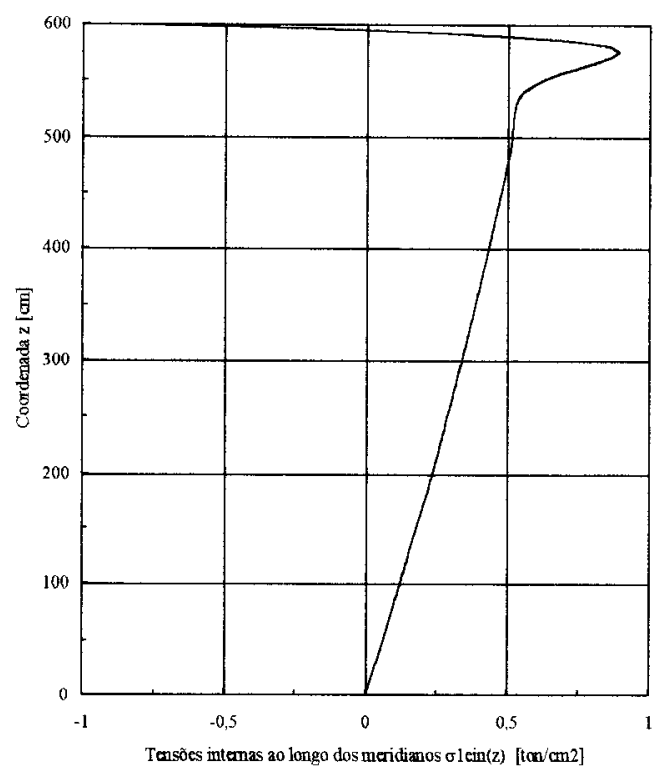

Eiementos Finitos

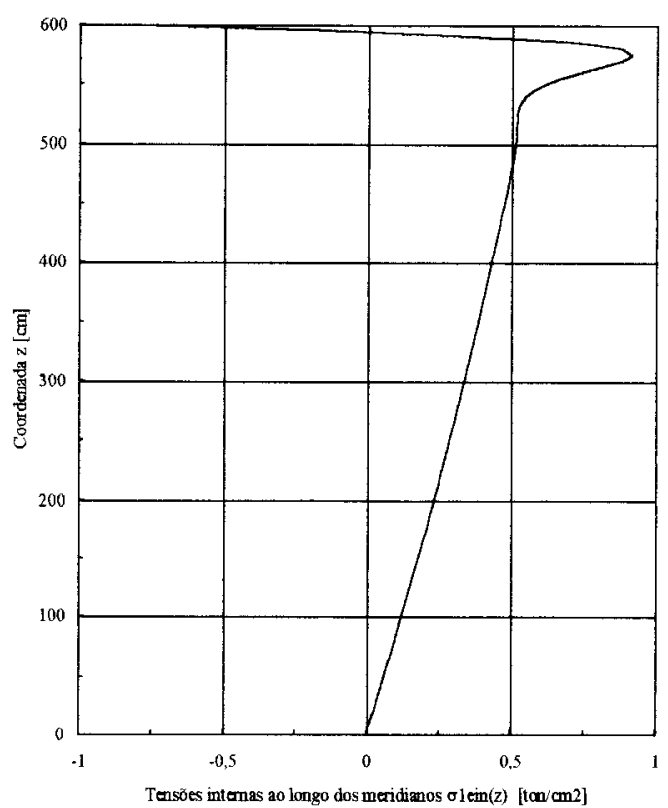

FIGURA 73 - Gráficos para os valores das tensões ao longo dos meridianos da superficie interna $\sigma$ lein( $(z)$ da casca cônica

Tearia

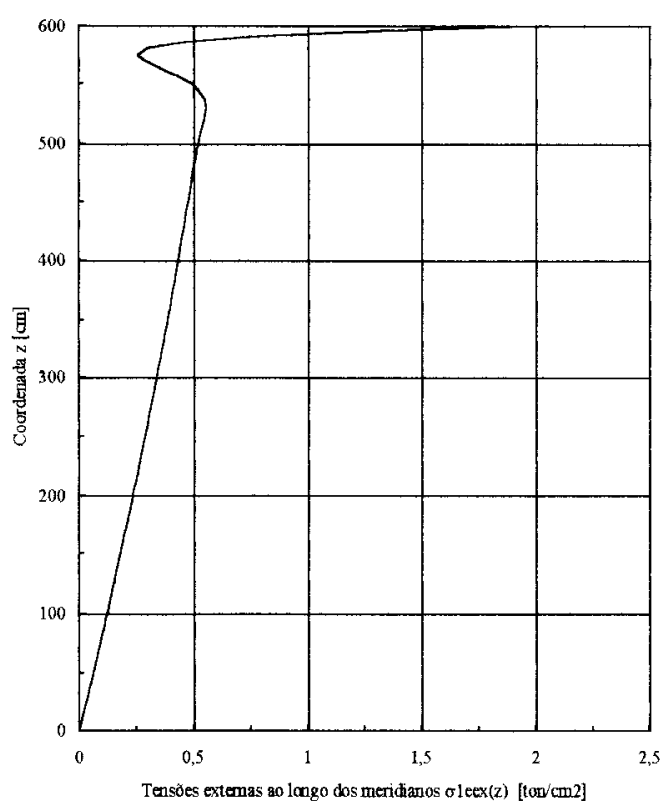

Elementos Finitos

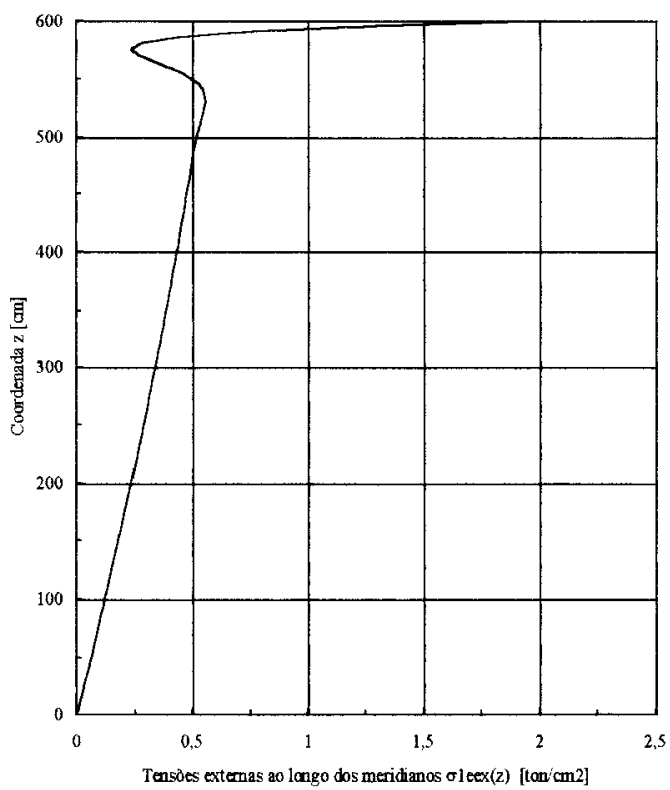

FIGURA 74 - Gráficos para os valores das tensões ao longo dos meridianos da superficie externa $\sigma$ leex $(\mathrm{z})$ da casca cônica 
E finalmente para as tensões atuantes ao longo dos paralelos da superficie interna e externa da casca cônica encontrou-se :

TABELA 22 - Valores para as tensões ao longo dos paralelos da superficie interna $\sigma 2 \operatorname{ein}(\mathrm{z})$ e externa $\sigma 2 \operatorname{eex}(\mathrm{z})$ da casca

\begin{tabular}{|c|c|c|c|}
\hline & \multicolumn{2}{|c|}{$\begin{array}{c}\sigma 2 \operatorname{ein}(\mathrm{z}) \\
{\left[\operatorname{ton} / \mathrm{cm}^{2}\right]}\end{array}$} & \multirow[b]{2}{*}{ Diferença } \\
\hline $\begin{array}{c}\mathrm{z} \\
{[\mathrm{cm}]}\end{array}$ & Teoria & $\begin{array}{c}\text { Elementos } \\
\text { Finitos }\end{array}$ & \\
\hline 600,00 & $-0,2652$ & $-0,2709$ & 0,0057 \\
\hline 595,00 & 0,0486 & 0,0361 & 0,0125 \\
\hline 590,00 & 0,3522 & 0,3406 & 0,0116 \\
\hline 585,00 & 0,6065 & 0,5995 & 0,0071 \\
\hline 580,00 & 0,7975 & 0,7958 & 0,0017 \\
\hline 575,00 & 0,9266 & 0,9291 & 0,0025 \\
\hline 570,00 & 1,0035 & 1,0081 & 0,0046 \\
\hline 565,00 & 1,0408 & 1,0453 & 0,0045 \\
\hline 560,00 & 1,0508 & 1,0536 & 0,0028 \\
\hline 555,00 & 1,0441 & 1,0442 & 0,0001 \\
\hline 550,00 & 1,0286 & 1,0260 & 0,0026 \\
\hline 545,00 & 1,0100 & 1,0050 & 0,0050 \\
\hline 540,00 & 0,9915 & 0,9848 & 0,0067 \\
\hline 535,00 & 0,9750 & 0,9673 & 0,0077 \\
\hline 530,00 & 0,9612 & 0,9532 & 0,0080 \\
\hline 525,00 & 0,9500 & 0,9422 & 0,0077 \\
\hline 520,00 & 0,9410 & 0,9337 & 0,0072 \\
\hline 515,00 & 0,9336 & 0,9270 & 0,0066 \\
\hline 510,00 & 0,9275 & 0,9215 & 0,0060 \\
\hline 505,00 & 0,9219 & 0,9165 & 0,0054 \\
\hline 500,00 & 0,9168 & 0,9117 & 0,0051 \\
\hline 495,00 & 0,9117 & 0,9068 & 0,0048 \\
\hline
\end{tabular}

\begin{tabular}{|c|c|c|}
\hline \multicolumn{2}{|c|}{$\begin{array}{c}\sigma 2 \text { eex(z) } \\
\text { [ton/cm }{ }^{2} \text { ] }\end{array}$} & \\
\cline { 1 - 2 } Teoria & $\begin{array}{c}\text { Elementos } \\
\text { Finitos }\end{array}$ & Diferença \\
\hline 0,6216 & 0,6074 & 0,0142 \\
\hline 0,4426 & 0,4252 & 0,0174 \\
\hline 0,4262 & 0,4076 & $\mathbf{0 , 0 1 8 6}$ \\
\hline 0,4984 & 0,4813 & 0,0171 \\
\hline 0,6072 & 0,5942 & 0,0130 \\
\hline 0,7194 & 0,7116 & 0,0079 \\
\hline 0,8168 & 0,8139 & 0,0029 \\
\hline 0,8917 & 0,8925 & 0,0008 \\
\hline 0,9431 & 0,9459 & 0,0028 \\
\hline 0,9739 & 0,9771 & 0,0032 \\
\hline 0,9885 & 0,9909 & 0,0023 \\
\hline 0,9917 & 0,9925 & 0,0007 \\
\hline 0,9876 & 0,9864 & 0,0011 \\
\hline 0,9793 & 0,9765 & 0,0028 \\
\hline 0,9693 & 0,9651 & 0,0041 \\
\hline 0,9589 & 0,9539 & 0,0050 \\
\hline 0,9491 & 0,9437 & 0,0054 \\
\hline 0,9402 & 0,9348 & 0,0054 \\
\hline 0,9322 & 0,9270 & 0,0052 \\
\hline 0,9251 & 0,9203 & 0,0048 \\
\hline 0,9186 & 0,9142 & 0,0044 \\
\hline 0,9125 & 0,9085 & 0,0040 \\
\hline & & \\
\hline & &
\end{tabular}



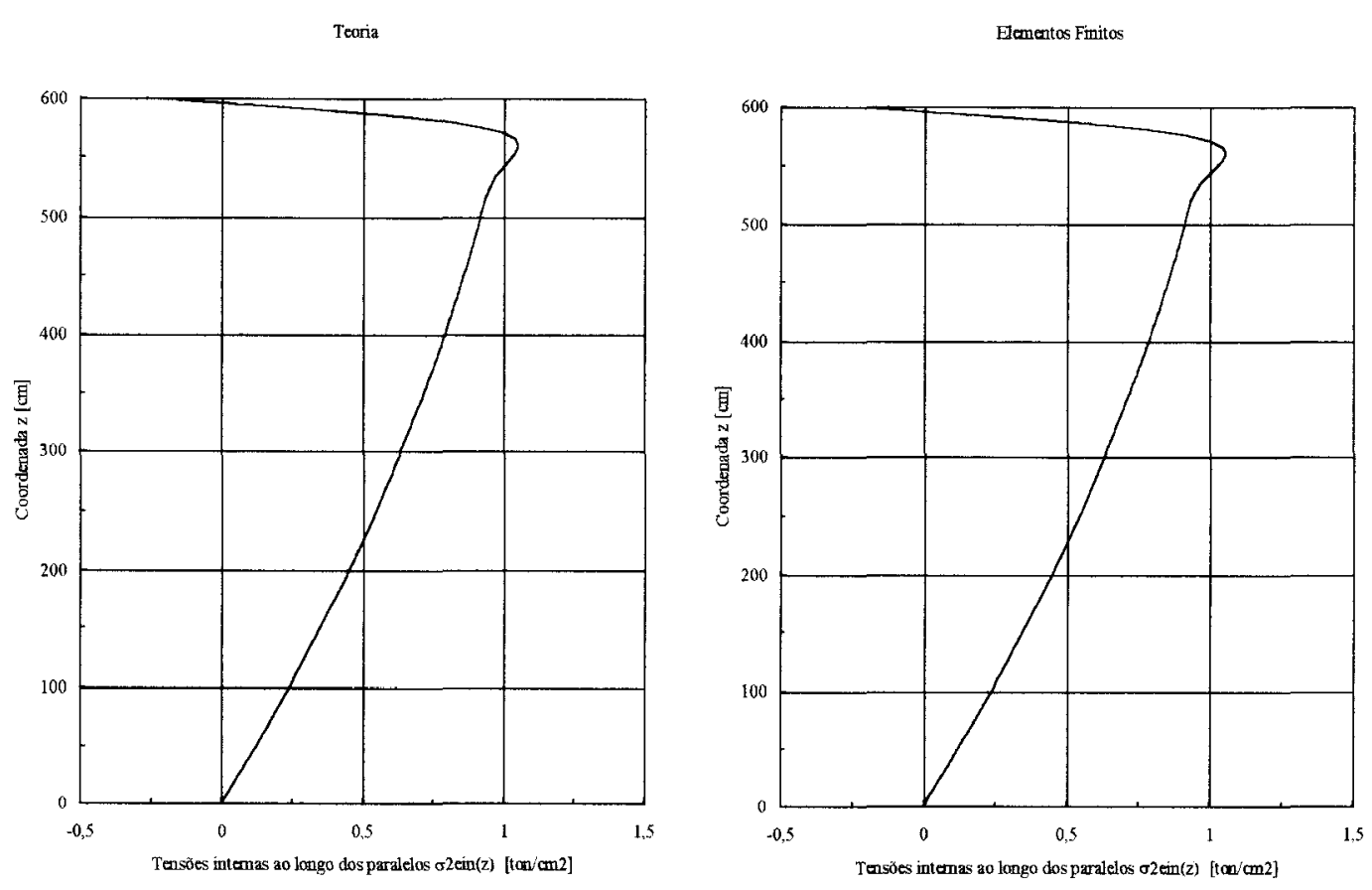

FIGURA 75 - Gráficos para os valores das tensões ao longo dos paralelos da superficie interna $\sigma 2$ ein(z) da casca cônica

Tearia

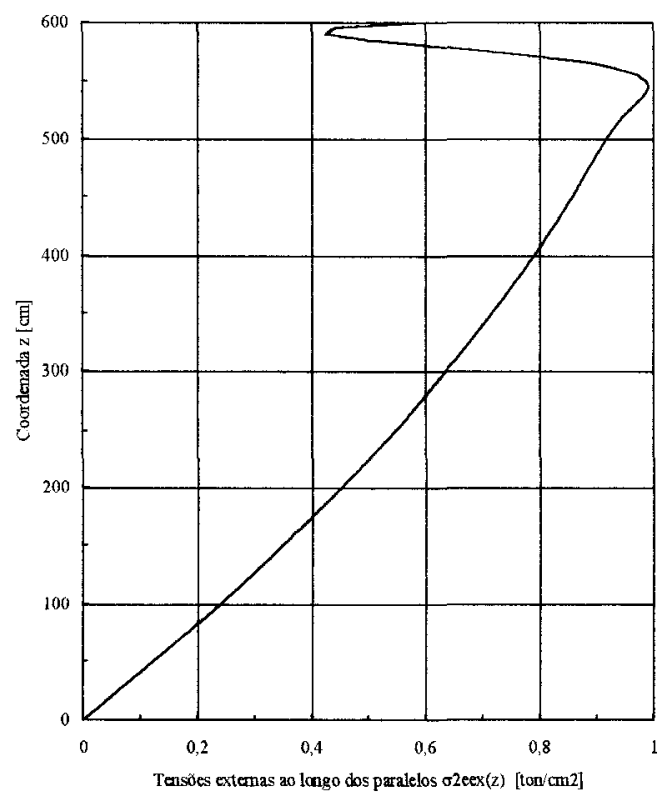

Elementos Finitos

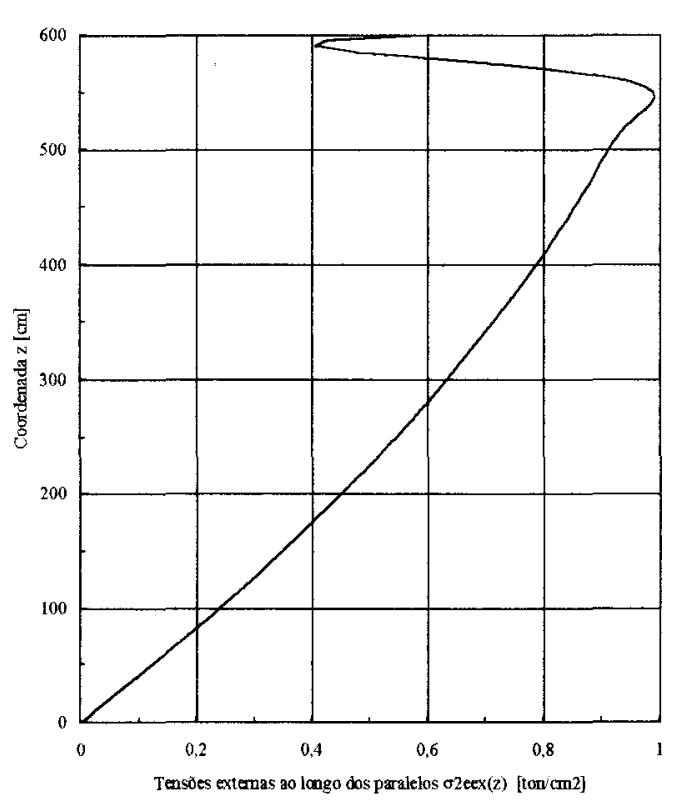

FIGURA 76 - Gráficos para os valores das tensões ao longo dos paralelos da superficie externa $\sigma 2 \operatorname{eex}(\mathrm{z})$ da casca cônica 
São apresentados abaixo os valores encontrados para as tensões de membrana atuantes ao longo dos meridianos e dos paralelos da superficie média da casca cônica para comparação :

TABELA 23 - Valores para as tensões teóricas de membrana ao longo dos meridianos $\sigma \operatorname{lm}(\mathrm{z})$ e paralelos $\sigma 2 \mathrm{~m}(\mathrm{z})$ da superficie média da casca

\begin{tabular}{|c|r|r|}
\hline $\begin{array}{c}\mathrm{z} \\
{[\mathrm{cm}]}\end{array}$ & $\begin{array}{c}\sigma 1 \mathrm{~m}(\mathrm{z}) \\
{\left[\text { ton } / \mathrm{cm}^{2}\right]}\end{array}$ & $\begin{array}{c}\sigma 2 \mathrm{~m}(\mathrm{z}) \\
{\left[\text { ton } / \mathrm{cm}^{2}\right]}\end{array}$ \\
\hline 600,00 & 0,5940 & 1,0182 \\
\hline 595,00 & 0,5904 & 1,0140 \\
\hline 590,00 & 0,5869 & 1,0096 \\
\hline 585,00 & 0,5833 & 1,0052 \\
\hline 580,00 & 0,5796 & 1,0007 \\
\hline 575,00 & 0,5760 & 0,9961 \\
\hline 570,00 & 0,5723 & 0,9915 \\
\hline 565,00 & 0,5686 & 0,9868 \\
\hline 560,00 & 0,5649 & 0,9820 \\
\hline 555,00 & 0,5612 & 0,9772 \\
\hline 550,00 & 0,5574 & 0,9723 \\
\hline
\end{tabular}

\begin{tabular}{|c|r|r|}
\hline $\begin{array}{c}\mathrm{z} \\
{[\mathrm{cm}]}\end{array}$ & $\begin{array}{c}\sigma 1 \mathrm{~m}(\mathrm{z}) \\
{\left[\text { ton } / \mathrm{cm}^{2}\right]}\end{array}$ & $\begin{array}{r}\sigma 2 \mathrm{~m}(\mathrm{z}) \\
{\left[\text { ton } / \mathrm{cm}^{2}\right]}\end{array}$ \\
\hline 545,00 & 0,5537 & 0,9673 \\
\hline 540,00 & 0,5498 & 0,9622 \\
\hline 535,00 & 0,5460 & 0,9571 \\
\hline 530,00 & 0,5422 & 0,9519 \\
\hline 525,00 & 0,5383 & 0,9466 \\
\hline 520,00 & 0,5344 & 0,9413 \\
\hline 515,00 & 0,5305 & 0,9359 \\
\hline 510,00 & 0,5265 & 0,9304 \\
\hline 505,00 & 0,5225 & 0,9249 \\
\hline 500,00 & 0,5185 & 0,9192 \\
\hline 495,00 & 0,5145 & 0,9135 \\
\hline
\end{tabular}

Pode-se ver abaixo nos gráficos com as tensões teóricas ao longo dos meridianos e paralelos da casca cônica, que as mesmas possuem seus valores praticamente coincidêntes abaixo da região de $\mathrm{z}=\mathrm{H}-\lambda \mathrm{z}=498,36[\mathrm{~cm}]$. 


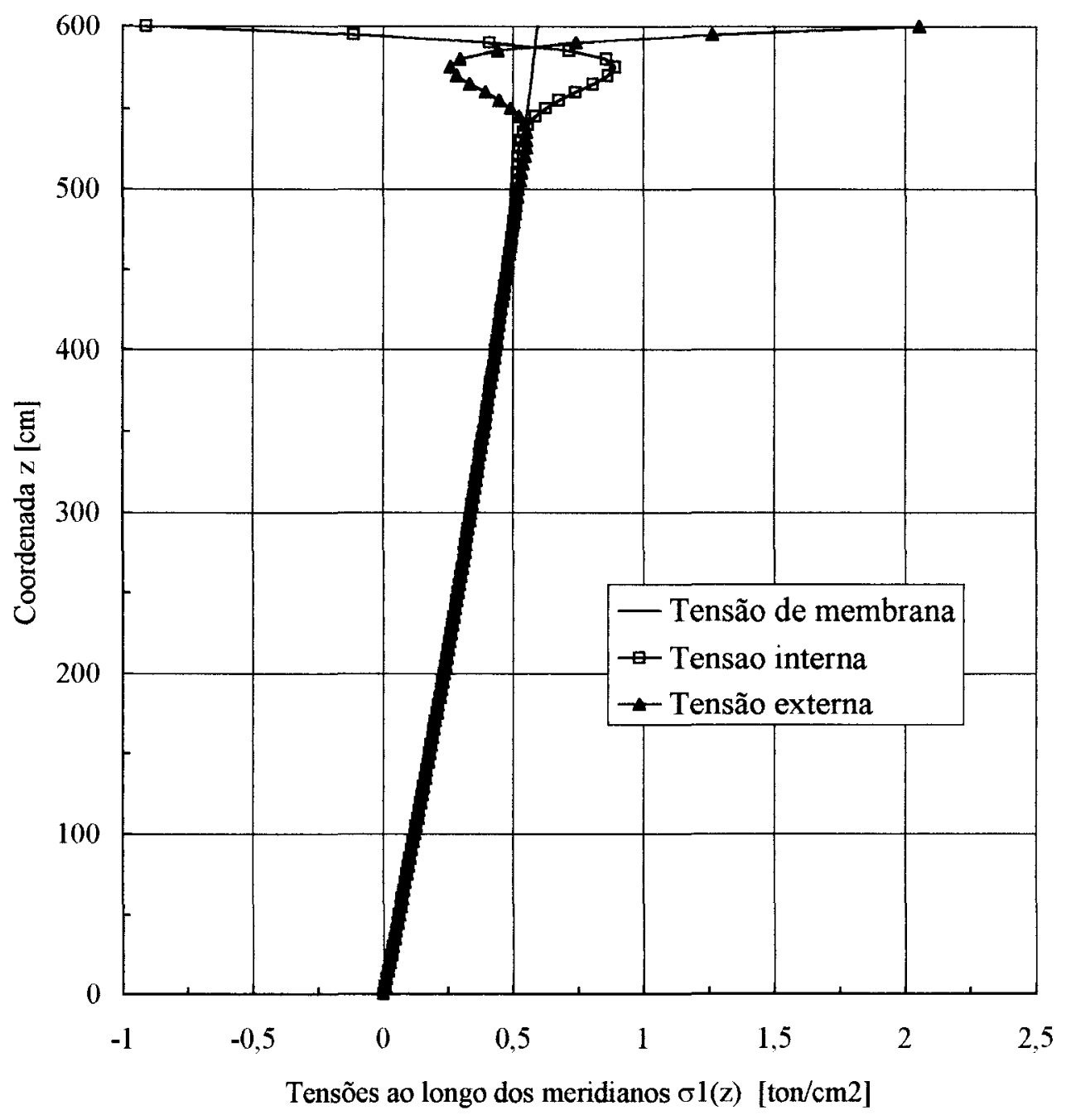

FIGURA 77 - Gráfico comparativo para os valores das tensões ao longo dos meridianos da casca cônica : $\sigma \operatorname{lm}(z), \sigma l \operatorname{ein}(z)$ e $\sigma l \operatorname{eex}(z)$ 
Teoria

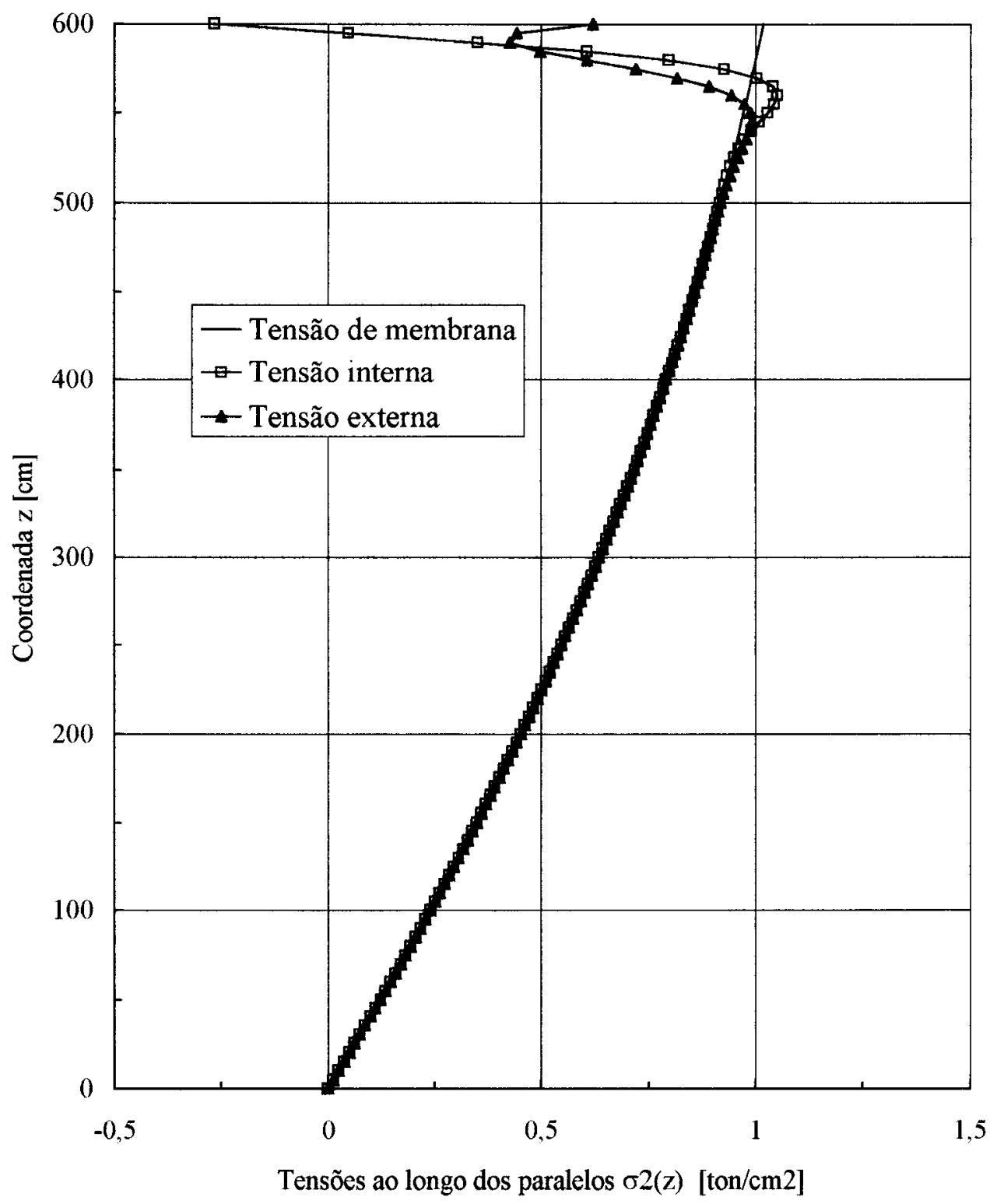

FIGURA 78 - Gráfico comparativo para os valores das tensões ao longo dos paralelos da casca cônica : $\sigma 2 \mathrm{~m}(\mathrm{z}), \sigma 2 \mathrm{ein}(\mathrm{z})$ e $\sigma 2 \operatorname{eex}(\mathrm{z})$ 


\subsubsection{CASCA CÔNICA COM BORDA SUPERIOR APOIADA FIXA}

Para os deslocamentos radiais e rotações ao longo do meridiano da superficie média da casca cônica encontrou-se :

TABELA 24 - Valores dos deslocamentos radiais $\xi_{\mathrm{a}}(\mathrm{z})$ e rotações $\phi \mathrm{a}(\mathrm{z})$

\begin{tabular}{|c|c|c|c|}
\hline & \multicolumn{2}{|c|}{$\begin{array}{l}\xi \mathrm{a}(\mathrm{z}) \\
{[\mathrm{cm}]}\end{array}$} & \\
\hline $\begin{array}{c}\mathrm{z} \\
{[\mathrm{cm}]}\end{array}$ & Teoria & \begin{tabular}{|c|} 
Elementos \\
Finitos
\end{tabular} & Diferença \\
\hline 600,00 & 0,0000 & 0,0000 & 0,0000 \\
\hline 595,00 & 0,0699 & 0,0693 & 0,0006 \\
\hline 590,00 & 0,1298 & 0,1293 & 0,0005 \\
\hline 585,00 & 0,1755 & 0,1752 & 0,0002 \\
\hline 580,00 & 0,2065 & 0,2065 & 0,0000 \\
\hline 575,00 & 0,2249 & 0,2248 & 0,0001 \\
\hline 570,00 & 0,2335 & 0,2332 & 0,0003 \\
\hline 565,00 & 0,2352 & 0,2344 & 0,0007 \\
\hline 560,00 & 0,2325 & 0,2313 & 0,0011 \\
\hline 555,00 & 0,2274 & 0,2259 & 0,0015 \\
\hline 550,00 & 0,2214 & 0,2197 & 0,0017 \\
\hline 545,00 & 0,2154 & 0,2135 & 0,0019 \\
\hline 540,00 & 0,2097 & 0,2079 & 0,0018 \\
\hline 535,00 & 0,2046 & 0,2029 & 0,0017 \\
\hline 530,00 & 0,2002 & 0,1987 & 0,0015 \\
\hline 525,00 & 0,1964 & 0,1950 & 0,0013 \\
\hline 520,00 & 0,1929 & 0,1918 & 0,0012 \\
\hline 515,00 & 0,1898 & 0,1888 & 0,0010 \\
\hline 510,00 & 0,1869 & 0,1860 & 0,0009 \\
\hline 505,00 & 0,1841 & 0,1833 & 0,0008 \\
\hline 500,00 & 0,1814 & 0,1806 & 0,0008 \\
\hline 495,00 & 0,1786 & 0,1779 & 0,0008 \\
\hline
\end{tabular}

\begin{tabular}{|c|c|c|}
\hline \multicolumn{2}{|c|}{$\begin{array}{c}\phi a(z) \\
\text { [radianos] }\end{array}$} & \multirow[b]{2}{*}{ Diferença } \\
\hline Teoria & $\begin{array}{c}\text { Elementos } \\
\text { Finitos } \\
\end{array}$ & \\
\hline$-0,0145$ & $-0,0144$ & 0,0001 \\
\hline$-0,0134$ & $-0,0134$ & 0,0000 \\
\hline$-0,0108$ & $-0,0108$ & 0,0001 \\
\hline$-0,0078$ & $-0,0078$ & 0,0001 \\
\hline$-0,0050$ & $-0,0050$ & 0,0000 \\
\hline$-0,0027$ & $-0,0027$ & 0,0000 \\
\hline$-0,0011$ & $-0,0010$ & 0,0001 \\
\hline 0,0000 & 0,0001 & 0,0001 \\
\hline 0,0007 & 0,0008 & 0,0001 \\
\hline 0,0010 & 0,0011 & 0,0001 \\
\hline 0,0011 & 0,0011 & 0,0000 \\
\hline 0,0011 & 0,0011 & 0,0000 \\
\hline 0,0010 & 0,0009 & 0,0000 \\
\hline 0,0008 & 0,0008 & 0,0000 \\
\hline 0,0007 & 0,0007 & 0,0000 \\
\hline 0,0006 & 0,0006 & 0,0000 \\
\hline 0,0005 & 0,0005 & 0,0000 \\
\hline 0,0005 & 0,0004 & 0,0000 \\
\hline 0,0005 & 0,0004 & 0,0000 \\
\hline 0,0004 & 0,0004 & 0,0000 \\
\hline 0,0004 & 0,0004 & 0,0000 \\
\hline 0,0004 & 0,0004 & 0,0000 \\
\hline
\end{tabular}



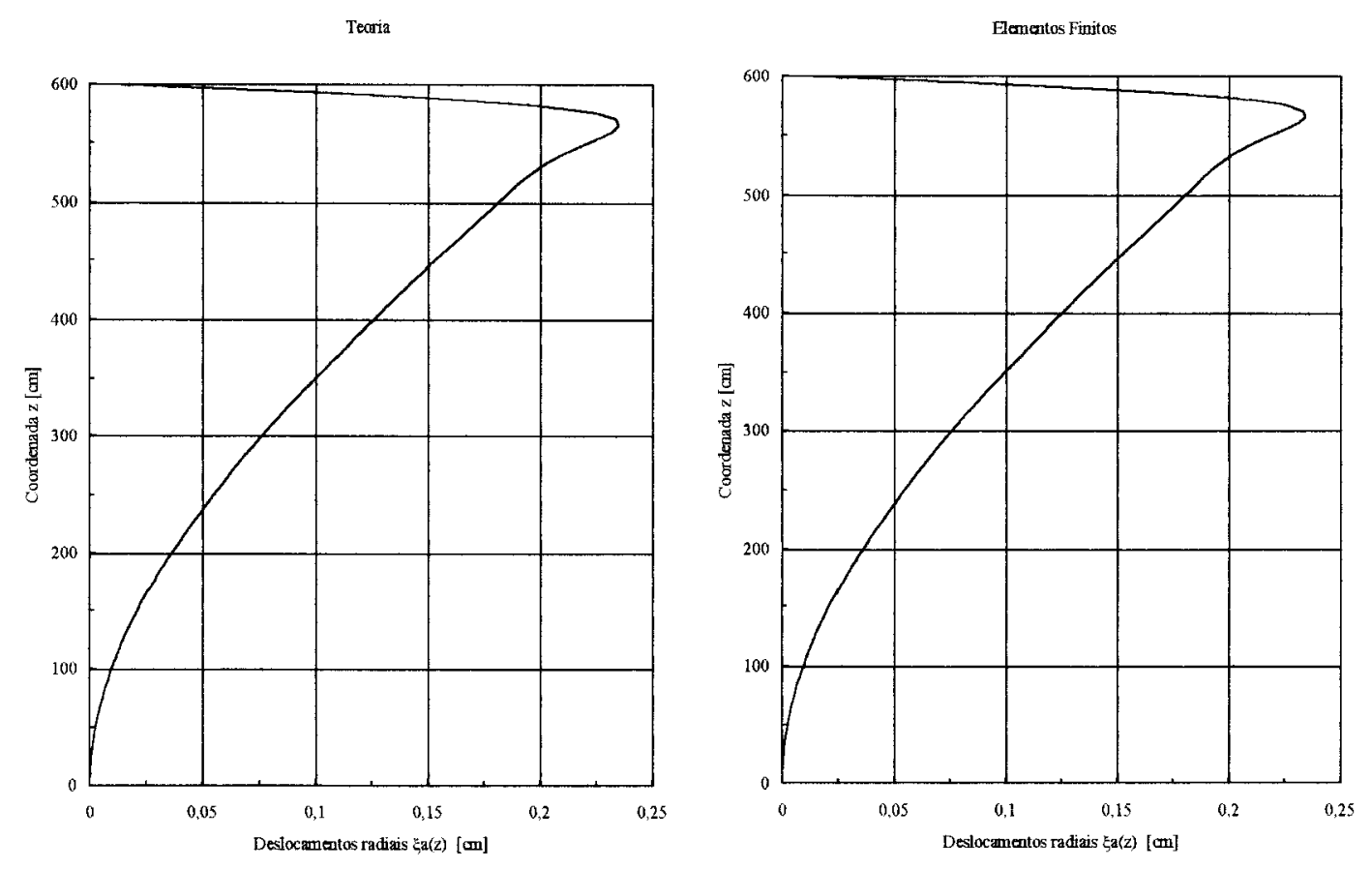

FIGURA 79 - Gráficos para os valores dos deslocamentos radiais $\xi \mathrm{a}(\mathrm{z})$ ao longo do meridiano da superfície média da casca cônica
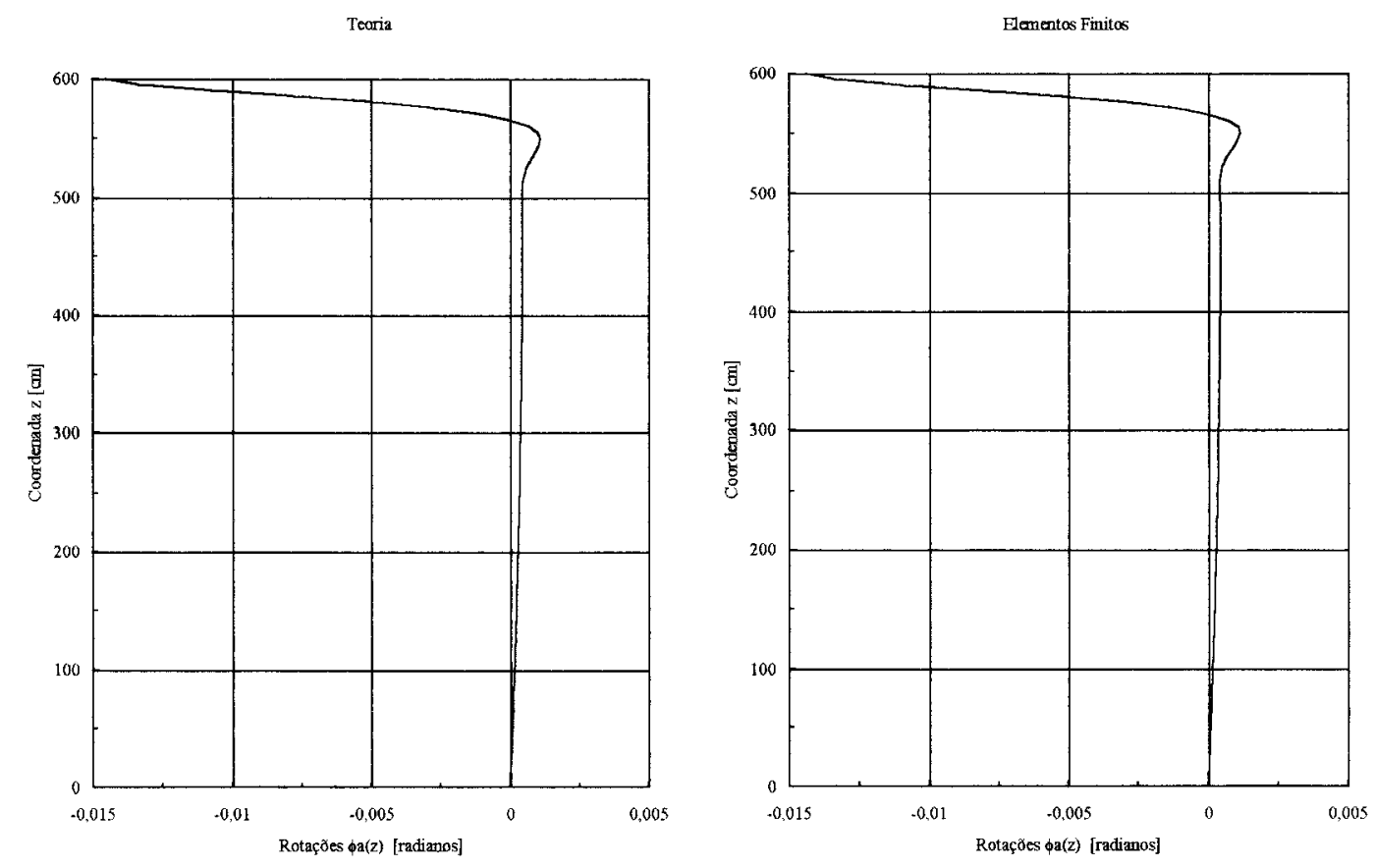

FIGURA 80 - Gráficos para os valores das rotações $\phi \mathrm{a}(\mathrm{z})$ ao longo do meridiano da superficie média da casca cônica 
Os momentos solicitantes ao longo dos paralelos e dos meridianos da superficie média da casca cônica e suas correspondentes diferenças absolutas foram :

TABELA 25 - Valores dos momentos ao longo dos paralelos

M1a(z) e dos meridianos M2a(z)

\begin{tabular}{|c|c|c|c|c|c|c|}
\hline & \multicolumn{2}{|c|}{$\begin{array}{c}\mathrm{M} 1 \mathrm{a}(\mathrm{z}) \\
{[\text { ton. } \mathrm{cm} / \mathrm{cm}]}\end{array}$} & & \multicolumn{2}{|c|}{$\begin{array}{c}\mathrm{M} 2 \mathrm{a}(\mathrm{z}) \\
{[\text { ton } \mathrm{cm} / \mathrm{cm}]}\end{array}$} & \\
\hline $\begin{array}{c}\mathrm{z} \\
{[\mathrm{cm}]}\end{array}$ & Teoria & $\begin{array}{c}\text { Elementos } \\
\text { Finitos } \\
\end{array}$ & Diferença & Teoria & $\begin{array}{c}\text { Elementos } \\
\text { Finitos }\end{array}$ & Diferença \\
\hline 600,00 & 0,0000 & 0,0003 & 0,0003 & $-0,0034$ & $-0,0029$ & 0,0005 \\
\hline 595,00 & $-0,0571$ & $-0,0581$ & 0,0010 & $-0,0203$ & $-0,0202$ & 0,0001 \\
\hline 590,00 & $-0,0796$ & $-0,0816$ & 0,0020 & $-0,0264$ & $-0,0267$ & 0,0003 \\
\hline 585,00 & $-0,0803$ & $-0,0827$ & 0,0024 & $-0,0259$ & $-0,0265$ & 0,0005 \\
\hline 580,00 & $-0,0692$ & $-0,0714$ & 0,0022 & $-0,0220$ & $-0,0225$ & 0,0005 \\
\hline 575,00 & $-0,0534$ & $-0,0549$ & 0,0015 & $-0,0167$ & $-0,0171$ & 0,0003 \\
\hline 570,00 & $-0,0373$ & $-0,0380$ & 0,0006 & $-0,0115$ & $-0,0116$ & 0,0001 \\
\hline 565,00 & $-0,0234$ & $-0,0232$ & 0,0002 & $-0,0071$ & $-0,0069$ & 0,0002 \\
\hline 560,00 & $-0,0126$ & $-0,0119$ & 0,0007 & $-0,0037$ & $-0,0034$ & 0,0003 \\
\hline 555,00 & $-0,0050$ & $-0,0040$ & 0,0010 & $-0,0014$ & $-0,0010$ & 0,0004 \\
\hline 550,00 & $-0,0002$ & 0,0008 & 0,0011 & 0,0001 & 0,0005 & 0,0004 \\
\hline 545,00 & 0,0024 & 0,0033 & 0,0009 & 0,0008 & 0,0012 & 0,0004 \\
\hline 540,00 & 0,0034 & 0,0041 & 0,0007 & 0,0011 & 0,0014 & 0,0003 \\
\hline 535,00 & 0,0035 & 0,0039 & 0,0004 & 0,0011 & 0,0014 & 0,0002 \\
\hline 530,00 & 0,0030 & 0,0032 & 0,0002 & 0,0010 & 0,0011 & 0,0001 \\
\hline 525,00 & 0,0024 & 0,0023 & 0,0000 & 0,0007 & 0,0008 & 0,0001 \\
\hline 520,00 & 0,0017 & 0,0015 & 0,0002 & 0,0005 & 0,0006 & 0,0001 \\
\hline 515,00 & 0,0010 & 0,0008 & 0,0002 & 0,0003 & 0,0004 & 0,0000 \\
\hline 510,00 & 0,0006 & 0,0004 & 0,0002 & 0,0002 & 0,0002 & 0,0000 \\
\hline 505,00 & 0,0002 & 0,0001 & 0,0002 & 0,0001 & 0,0001 & 0,0001 \\
\hline 500,00 & 0,0000 & $-0,0001$ & 0,0001 & 0,0000 & 0,0001 & 0,0001 \\
\hline 495,00 & $-0,0001$ & $-0,0002$ & 0,0001 & 0,0000 & 0,0001 & 0,0001 \\
\hline
\end{tabular}



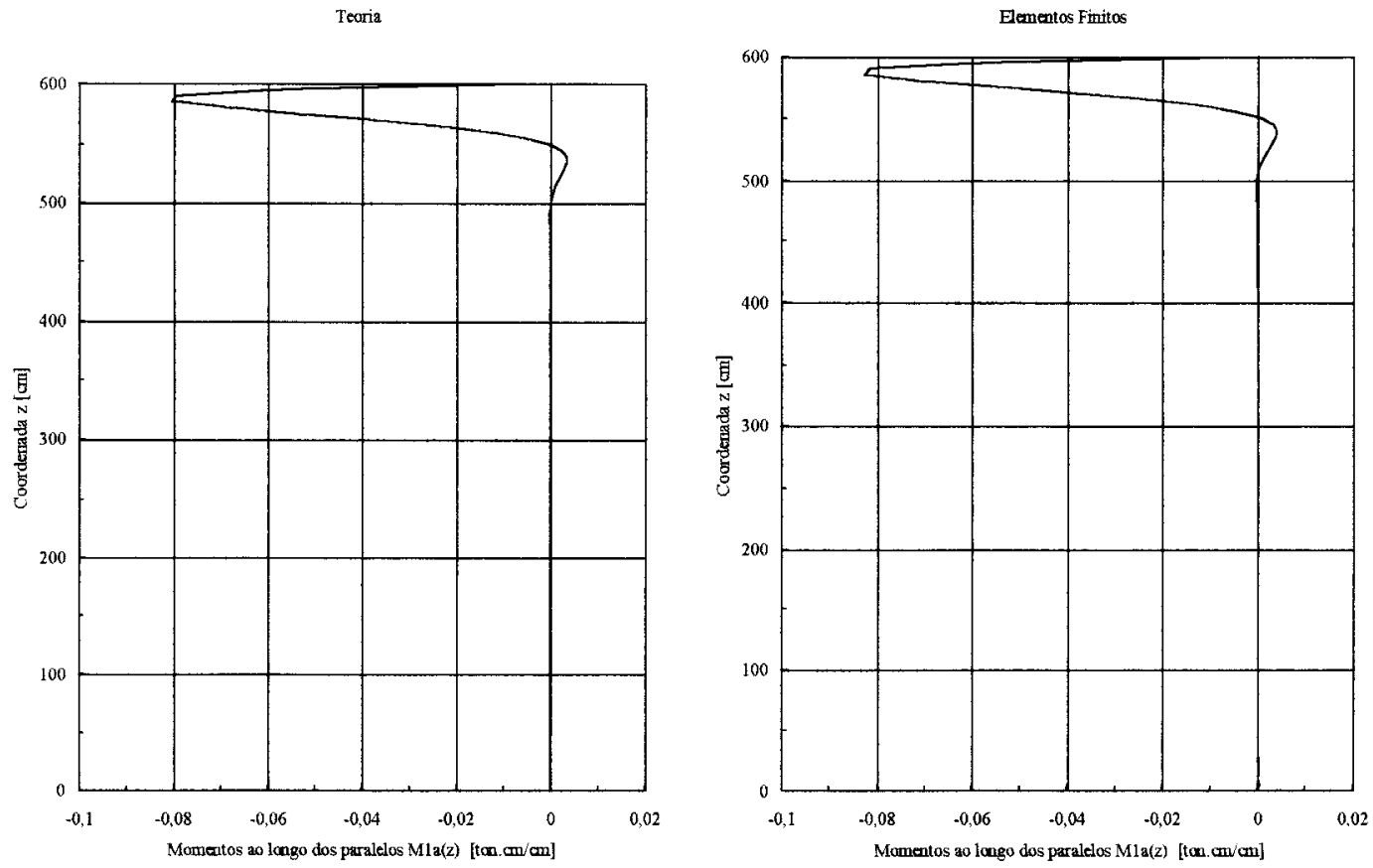

FIGURA 81 - Gráficos para os valores dos momentos ao longo dos paralelos Mla(z) da superficie média da casca cônica
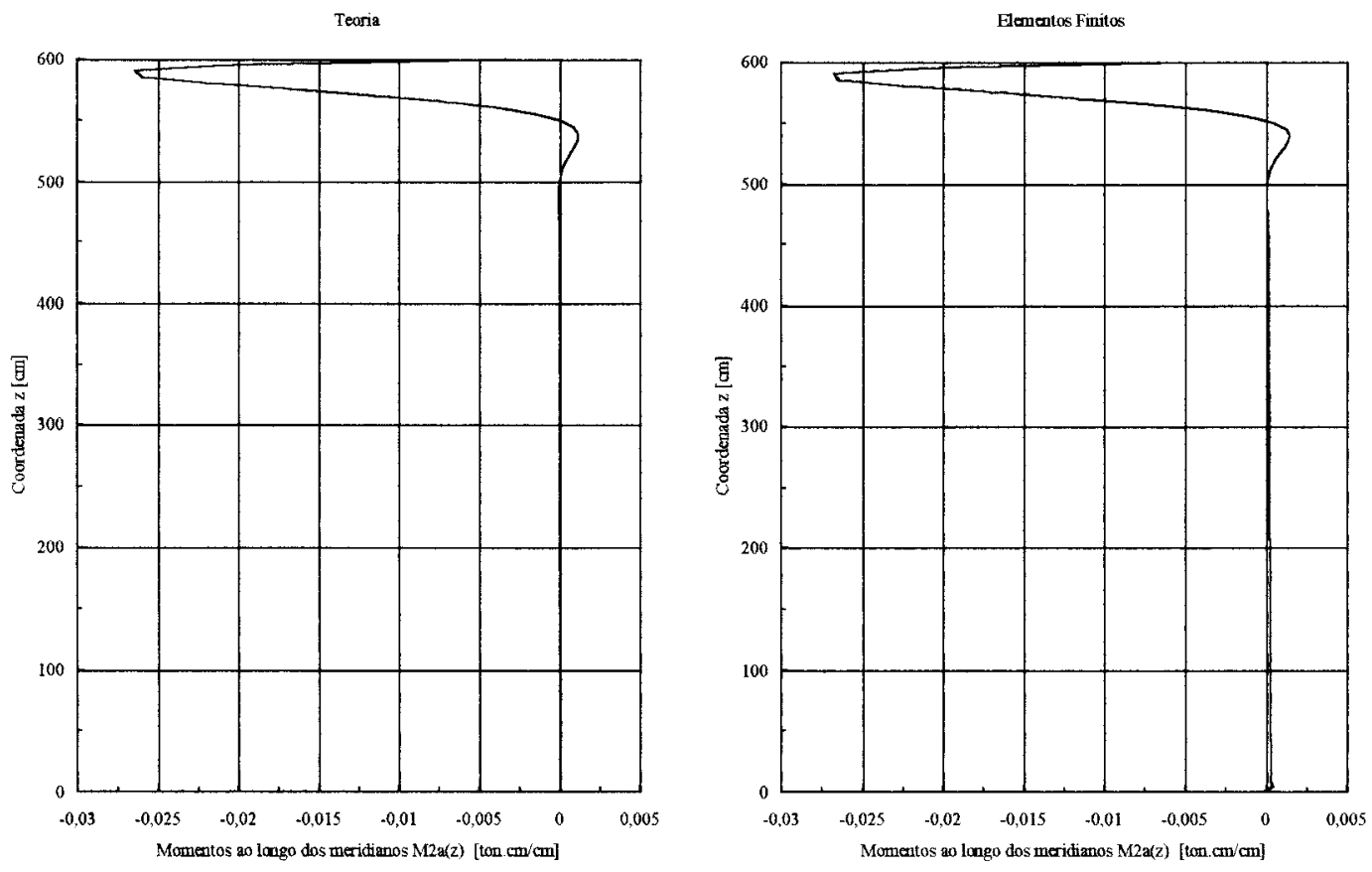

FIGURA 82 - Gráficos para os valores dos momentos ao longo dos meridianos $\mathrm{M} 2 \mathrm{a}(\mathrm{z})$ da superfície média da casca cônica 
Para as tensões atuantes ao longo dos meridianos da superficie interna e externa da casca cônica obteve-se :

TABELA 26 - Valores para as tensões ao longo dos meridianos da superficie interna $\sigma$ lain(z) e externa $\sigma$ laex(z) da casca

\begin{tabular}{|c|c|c|c|}
\cline { 2 - 3 } \multicolumn{1}{c|}{} & \multicolumn{2}{c|}{\begin{tabular}{c}
\multicolumn{1}{c}{} \\
\multicolumn{1}{c|}{$\left[\right.$ ton $\left./ \mathrm{cm}^{2}\right]$}
\end{tabular}} & \multicolumn{1}{c}{} \\
\hline $\begin{array}{c}z \\
{[\mathrm{~cm}]}\end{array}$ & Teoria & $\begin{array}{c}\text { Elementos } \\
\text { Finitos }\end{array}$ & Diferença \\
\hline 600,00 & 0,5828 & 0,5393 & $\mathbf{0 , 0 4 3 4}$ \\
\hline 595,00 & 0,9279 & 0,9331 & 0,0053 \\
\hline 590,00 & 1,0630 & 1,0759 & 0,0129 \\
\hline 585,00 & 1,0658 & 1,0819 & 0,0160 \\
\hline 580,00 & 0,9969 & 1,0113 & 0,0144 \\
\hline 575,00 & 0,8988 & 0,9084 & 0,0097 \\
\hline 570,00 & 0,7985 & 0,8022 & 0,0037 \\
\hline 565,00 & 0,7108 & 0,7089 & 0,0019 \\
\hline 560,00 & 0,6418 & 0,6358 & 0,0060 \\
\hline 555,00 & 0,5922 & 0,5839 & 0,0083 \\
\hline 550,00 & 0,5594 & 0,5505 & 0,0088 \\
\hline 545,00 & 0,5398 & 0,5316 & 0,0081 \\
\hline 540,00 & 0,5295 & 0,5228 & 0,0067 \\
\hline 535,00 & 0,5251 & 0,5200 & 0,0050 \\
\hline 530,00 & 0,5239 & 0,5204 & 0,0035 \\
\hline 525,00 & 0,5240 & 0,5218 & 0,0022 \\
\hline 520,00 & 0,5243 & 0,5229 & 0,0014 \\
\hline 515,00 & 0,5241 & 0,5230 & 0,0011 \\
\hline 510,00 & 0,5230 & 0,5220 & 0,0010 \\
\hline 505,00 & 0,5211 & 0,5199 & 0,0012 \\
\hline 500,00 & 0,5184 & 0,5169 & 0,0015 \\
\hline 495,00 & 0,5151 & 0,5133 & 0,0018 \\
\hline
\end{tabular}

\begin{tabular}{|c|c|c|}
\hline \multicolumn{2}{|c|}{$\begin{array}{l}\sigma l \mathrm{aex}(\mathrm{z}) \\
{\left[\mathrm{ton} / \mathrm{cm}^{2}\right]}\end{array}$} & \\
\hline Teoria & $\begin{array}{c}\text { Elementos } \\
\text { Finitos }\end{array}$ & Diferença \\
\hline 0,5828 & 0,5429 & $\mathbf{0 , 0 3 9 9}$ \\
\hline 0,2424 & 0,2359 & 0,0065 \\
\hline 0,1080 & 0,0972 & 0,0108 \\
\hline 0,1025 & 0,0896 & 0,0130 \\
\hline 0,1665 & 0,1545 & 0,0119 \\
\hline 0,2579 & 0,2494 & 0,0085 \\
\hline 0,3505 & 0,3464 & 0,0041 \\
\hline 0,4300 & 0,4300 & 0,0000 \\
\hline 0,4906 & 0,4935 & 0,0028 \\
\hline 0,5320 & 0,5361 & 0,0042 \\
\hline 0,5565 & 0,5606 & 0,0041 \\
\hline 0,5680 & 0,5711 & 0,0031 \\
\hline 0,5704 & 0,5719 & 0,0016 \\
\hline 0,5669 & 0,5668 & 0,0001 \\
\hline 0,5603 & 0,5587 & 0,0016 \\
\hline 0,5523 & 0,5497 & 0,0026 \\
\hline 0,5442 & 0,5410 & 0,0033 \\
\hline 0,5367 & 0,5331 & 0,0035 \\
\hline 0,5299 & 0,5264 & 0,0035 \\
\hline 0,5239 & 0,5207 & 0,0032 \\
\hline 0,5186 & 0,5158 & 0,0029 \\
\hline 0,5139 & 0,5114 & 0,0025 \\
\hline
\end{tabular}


Teoria

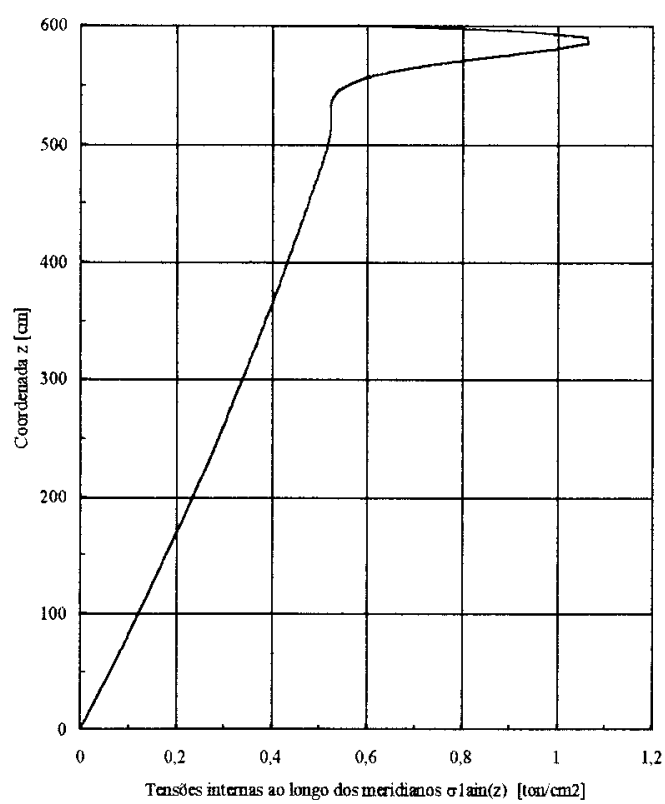

Flementos Finitos

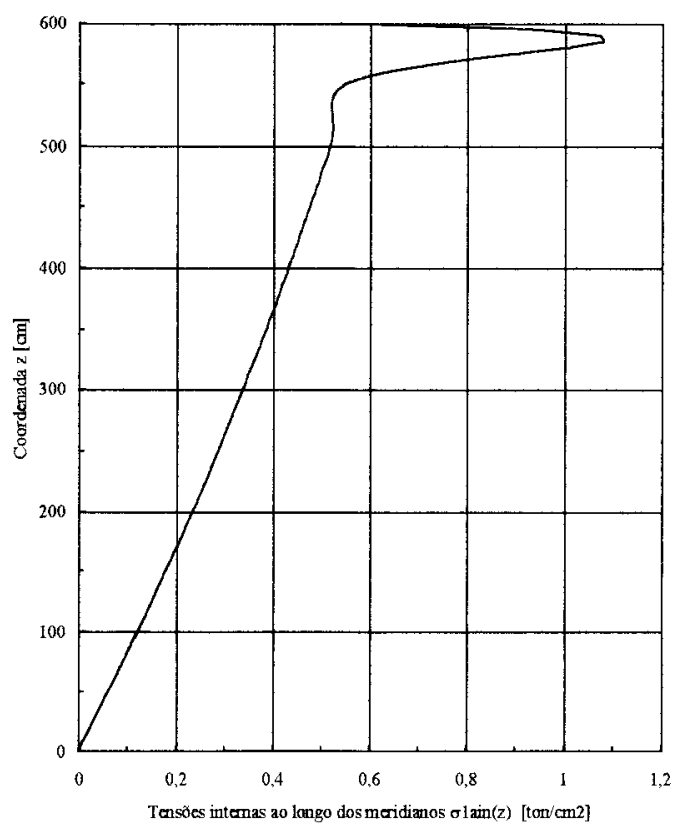

FIGURA 83 - Gráficos para os valores das tensões ao longo dos meridianos da superficie interna $\sigma$ lain(z) da casca cônica
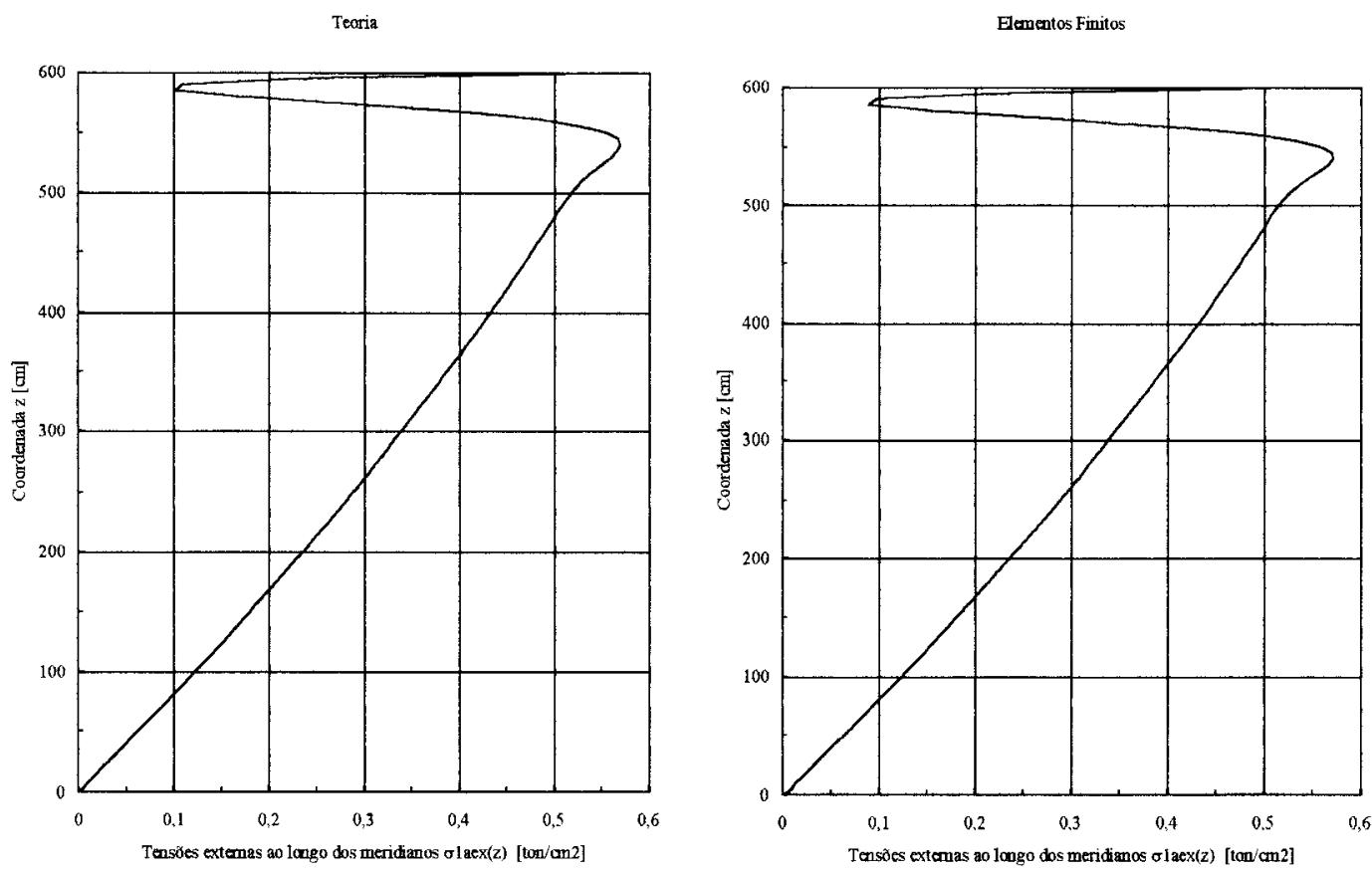

FIGURA 84 - Gráficos para os valores das tensões ao longo dos meridianos da superficie externa $\sigma$ laex(z) da casca cônica 
E, para as tensões atuantes ao longo dos paralelos da superficie interna e externa da casca cônica, encontrou-se :

TABELA 27 - Valores para as tensões ao longo dos paralelos da superficie interna $\sigma 2 \operatorname{ain}(z)$ e externa $\sigma 2 a e x(z)$ da casca

\begin{tabular}{|c|r|r|r|}
\cline { 2 - 3 } \multicolumn{1}{c|}{} & \multicolumn{2}{c|}{\begin{tabular}{c}
\multicolumn{1}{c}{} \\
[tonin(z)
\end{tabular}} & \multicolumn{1}{c}{$\left.\mathrm{cm}^{2}\right]$} \\
\hline $\begin{array}{c}z \\
{[\mathrm{~cm}]}\end{array}$ & Teoria & $\begin{array}{c}\text { Elementos } \\
\text { Finitos }\end{array}$ & Diferença \\
\hline 600,00 & 0,1986 & 0,1796 & $\mathbf{0 , 0 1 9 0}$ \\
\hline 595,00 & 0,5504 & 0,5413 & 0,0091 \\
\hline 590,00 & 0,8029 & 0,7967 & 0,0062 \\
\hline 585,00 & 0,9655 & 0,9635 & 0,0020 \\
\hline 580,00 & 1,0562 & 1,0574 & 0,0012 \\
\hline 575,00 & 1,0947 & 1,0972 & 0,0024 \\
\hline 570,00 & 1,0991 & 1,1010 & 0,0018 \\
\hline 565,00 & 1,0839 & 1,0839 & 0,0000 \\
\hline 560,00 & 1,0597 & 1,0573 & 0,0024 \\
\hline 555,00 & 1,0333 & 1,0286 & 0,0047 \\
\hline 550,00 & 1,0089 & 1,0025 & 0,0064 \\
\hline 545,00 & 0,9882 & 0,9807 & 0,0075 \\
\hline 540,00 & 0,9718 & 0,9639 & 0,0079 \\
\hline 535,00 & 0,9592 & 0,9515 & 0,0078 \\
\hline 530,00 & 0,9497 & 0,9424 & 0,0073 \\
\hline 525,00 & 0,9425 & 0,9358 & 0,0066 \\
\hline 520,00 & 0,9366 & 0,9307 & 0,0060 \\
\hline 515,00 & 0,9316 & 0,9262 & 0,0054 \\
\hline 510,00 & 0,9270 & 0,9220 & 0,0050 \\
\hline 505,00 & 0,9224 & 0,9176 & 0,0048 \\
\hline 500,00 & 0,9176 & 0,9129 & 0,0047 \\
\hline 495,00 & 0,9126 & 0,9079 & 0,0047 \\
\hline & & & \\
\hline
\end{tabular}

\begin{tabular}{|c|c|c|}
\hline \multicolumn{2}{|c|}{$\begin{array}{l}\sigma 2 \mathrm{aex}(\mathrm{z}) \\
{\left[\operatorname{ton} / \mathrm{cm}^{2}\right]}\end{array}$} & \\
\hline Teoria & $\begin{array}{c}\text { Elementos } \\
\text { Finitos }\end{array}$ & Diferença \\
\hline 0,1578 & 0,1451 & 0,0127 \\
\hline 0,3072 & 0,2988 & 0,0084 \\
\hline 0,4859 & 0,4758 & 0,0100 \\
\hline 0,6542 & 0,6460 & 0,0082 \\
\hline 0,7923 & 0,7875 & 0,0047 \\
\hline 0,8938 & 0,8925 & 0,0013 \\
\hline 0,9606 & 0,9616 & 0,0011 \\
\hline 0,9986 & 1,0006 & 0,0020 \\
\hline 1,0150 & 1,0167 & 0,0017 \\
\hline 1,0168 & 1,0172 & 0,0004 \\
\hline 1,0098 & 1,0086 & 0,0012 \\
\hline 0,9983 & 0,9955 & 0,0029 \\
\hline 0,9854 & 0,9812 & 0,0042 \\
\hline 0,9727 & 0,9677 & 0,0051 \\
\hline 0,9613 & 0,9558 & 0,0055 \\
\hline 0,9513 & 0,9458 & 0,0056 \\
\hline 0,9428 & 0,9375 & 0,0053 \\
\hline 0,9355 & 0,9305 & 0,0049 \\
\hline 0,9290 & 0,9245 & 0,0045 \\
\hline 0,9232 & 0,9190 & 0,0041 \\
\hline 0,9176 & 0,9138 & 0,0038 \\
\hline 0,9122 & 0,9086 & 0,0035 \\
\hline
\end{tabular}



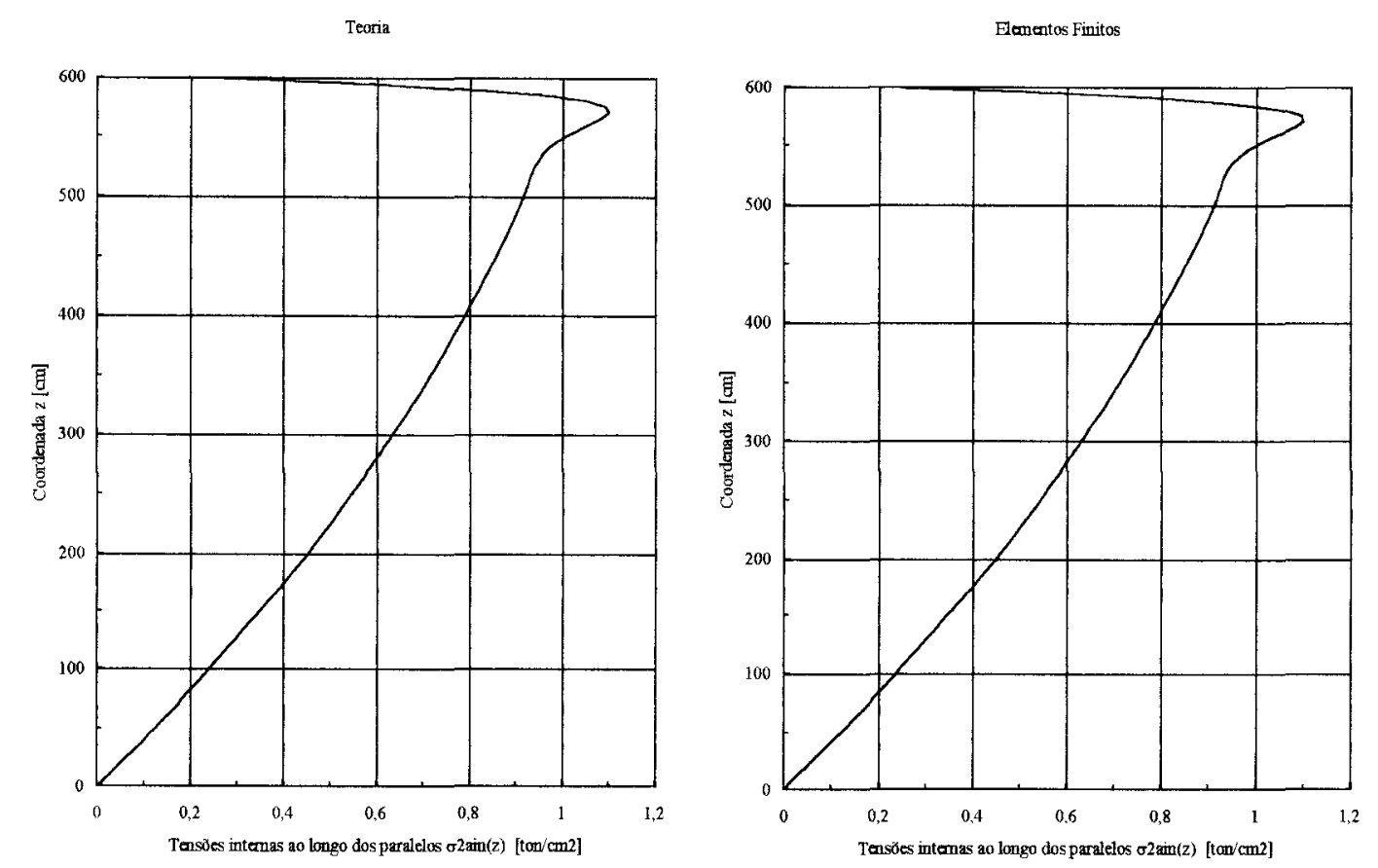

FIGURA 85 - Gráficos para os valores das tensões ao longo dos paralelos da superficie interna $\sigma 2$ ain( $(\mathrm{z})$ da casca cônica
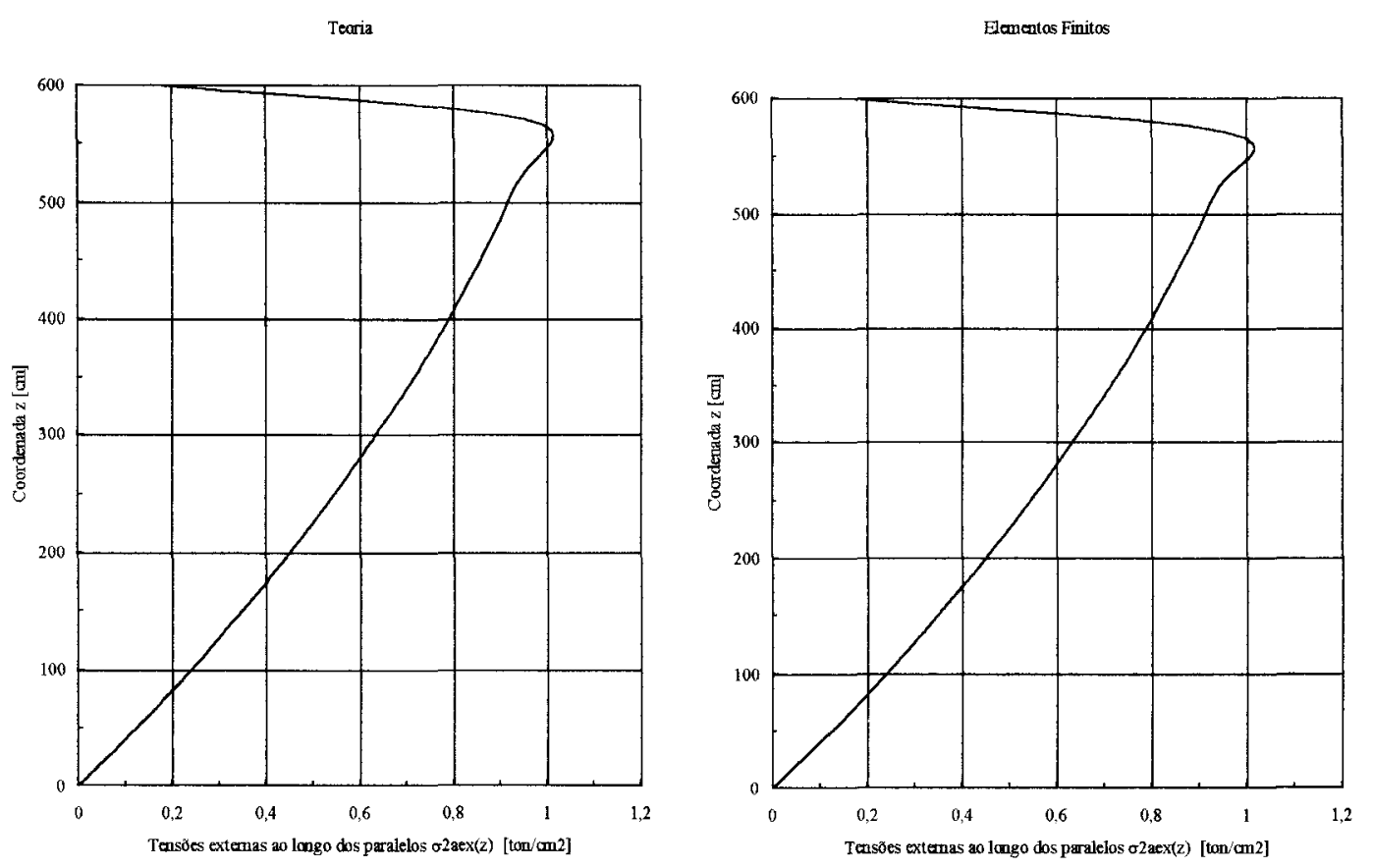

FIGURA 86 - Gráficos para os valores das tensões ao longo dos paralelos da superficie externa $\sigma 2 a e x(z)$ da casca cônica 
Observar, nos gráficos abaixo, que as tensões teóricas ao longo dos meridianos e paralelos da casca cônica, como para borda engastada, possuem seus valores praticamente coincidêntes com as tensões de membrana para a região abaixo de $z=H-\lambda z=498,36[\mathrm{~cm}]$.

\section{Teoria}

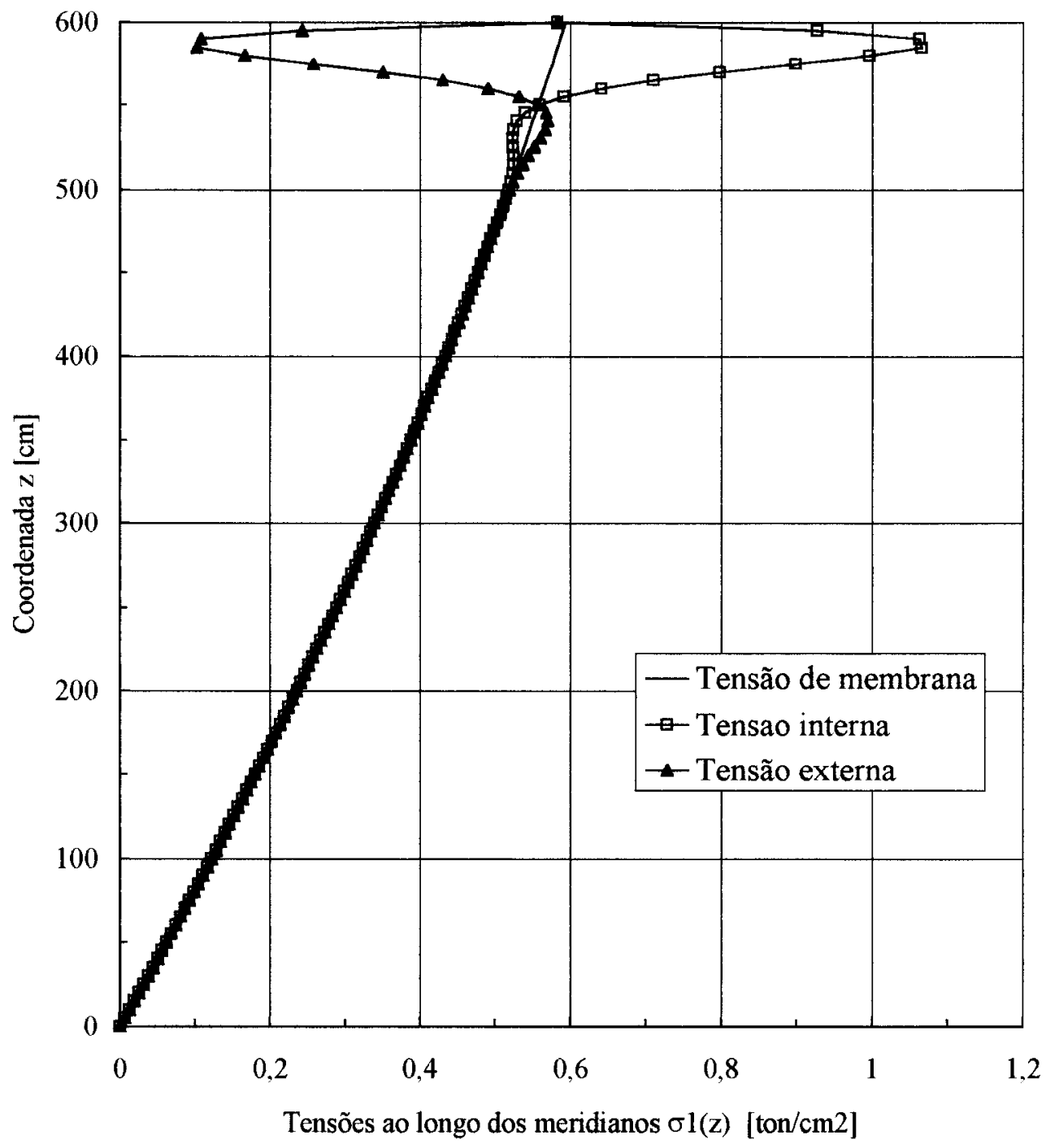

FIGURA 87 - Gráfico comparativo para os valores das tensões ao longo dos meridianos da casca cônica : $\sigma 1 \mathrm{~m}(\mathrm{z}), \sigma \operatorname{lain}(\mathrm{z})$ e $\sigma \operatorname{laex}(\mathrm{z})$ 
Teoria

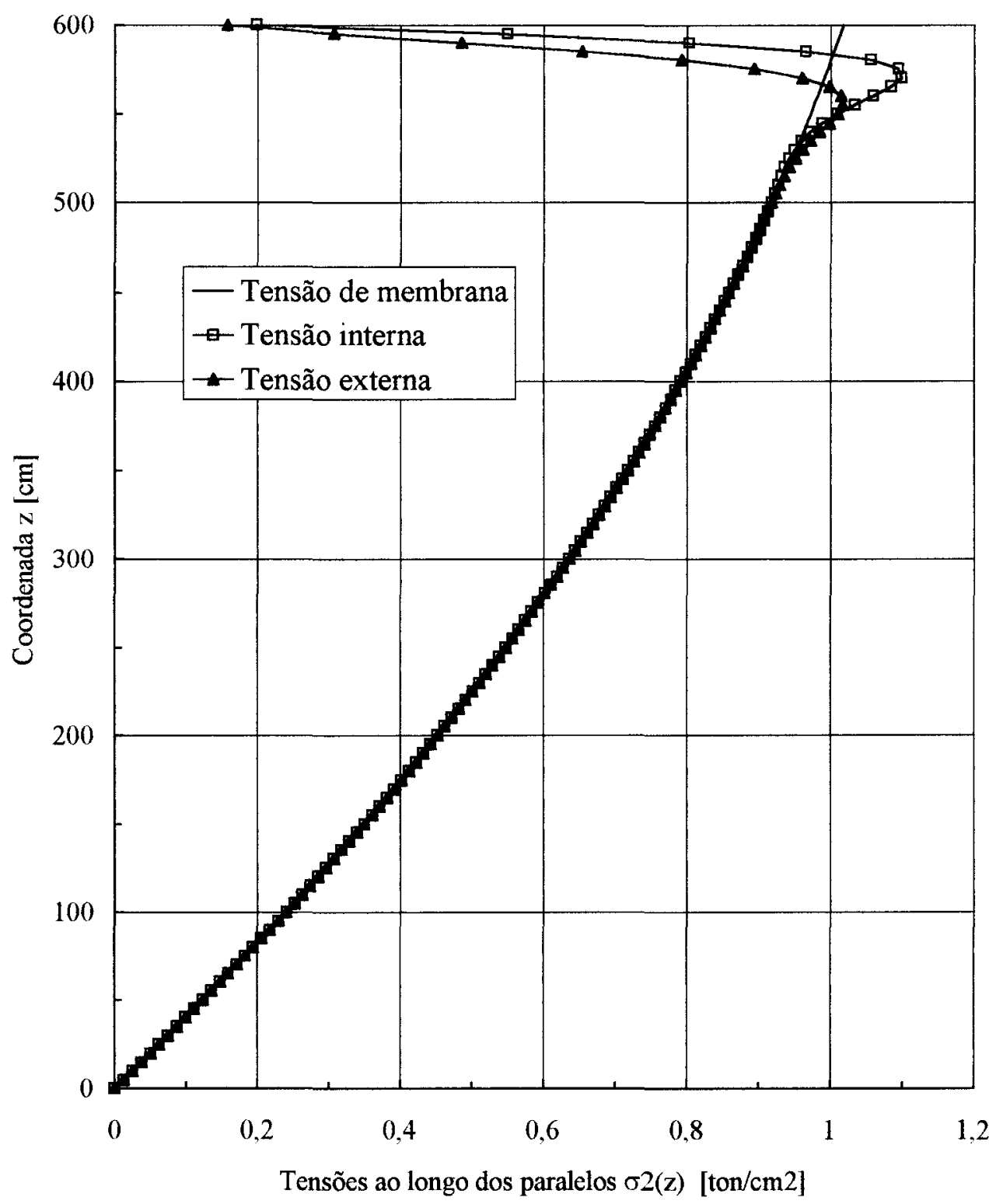

FIGURA 88 - Gráfico comparativo para os valores das tensões ao longo dos paralelos da casca cônica : $\sigma 2 \mathrm{~m}(\mathrm{z}), \sigma 2 \mathrm{ain}(\mathrm{z})$ e $\sigma 2 \mathrm{aex}(\mathrm{z})$ 


\subsection{CALCULANDO E OBTENDO RESULTADOS PARA A CASCA CÔNICA INCLINAÇÃO $\theta \mathrm{r}=60^{\circ}$ COM A HORIZONTAL}

Através das equações teóricas do capítulo 2 para casca cônica, e com os dados abaixo :

\begin{tabular}{|c|c|c|c|c|c|c|}
\hline $\begin{array}{c}\mathrm{E} \\
{\left[\text { ton } / \mathrm{cm}^{2}\right]}\end{array}$ & $\begin{array}{c}v \\
(\text { Aço) }\end{array}$ & $\begin{array}{c}\gamma \\
\left.\text { [ton } / \mathrm{cm}^{3}\right]\end{array}$ & $\begin{array}{c}\theta \mathrm{r} \\
\text { [graus] }\end{array}$ & $\begin{array}{c}\alpha \mathrm{r} \\
\text { [graus] }]\end{array}$ & $\begin{array}{c}\mathrm{D} \\
{[\mathrm{cm}]}\end{array}$ & $\begin{array}{c}\mathrm{r} \\
{[\mathrm{cm}]}\end{array}$ \\
\hline 2100 & 0,3 & $1,00 \mathrm{E}-06$ & 60 & 30 & 1200 & 600 \\
\hline
\end{tabular}

\begin{tabular}{|c|c|c|c|}
\hline $\begin{array}{c}\mathrm{R} \\
{[\mathrm{cm}]}\end{array}$ & $\begin{array}{c}\mathrm{H} \\
{[\mathrm{cm}]}\end{array}$ & $\begin{array}{c}\mathrm{h} \\
{[\mathrm{cm}]}\end{array}$ & $\begin{array}{c}\mathrm{t} \\
{[\mathrm{cm}]}\end{array}$ \\
\hline 692,82 & 1039,23 & 1200 & 1 \\
\hline
\end{tabular}

Encontram-se

\begin{tabular}{|c|c|c|c|c|c|c|}
\hline $\begin{array}{c}\beta \\
{\left[\text { ton } / \mathrm{cm}^{3}\right]}\end{array}$ & $\begin{array}{c}\mathrm{B} \\
{[\text { ton } / \mathrm{cm}]}\end{array}$ & $\begin{array}{c}\alpha \\
{\left[\mathrm{cm}^{-1}\right]}\end{array}$ & $\begin{array}{c}\xi \mathrm{h} \\
{\left[\mathrm{cm}^{2} / \text { ton }\right]}\end{array}$ & $\begin{array}{c}\xi \mathrm{m} \\
{[\mathrm{cm} / \text { ton }]}\end{array}$ & $\begin{array}{c}\phi \mathrm{h} \\
{[\mathrm{cm} / \text { ton }]}\end{array}$ & $\begin{array}{c}\phi \mathrm{m} \\
{\left[\text { ton }^{-1}\right]}\end{array}$ \\
\hline 0,0044 & 192,3077 & 0,0488 & 16,7434 & 0,9442 & 0,9442 & 0,1065 \\
\hline
\end{tabular}

\begin{tabular}{|c|c|c|}
\hline $\begin{array}{c}\text { Mce } \\
\text { [ton } . \mathrm{cm} / \mathrm{cm}]\end{array}$ & $\begin{array}{c}\text { Hce } \\
{[\text { ton } / \mathrm{cm}]}\end{array}$ & $\begin{array}{c}\text { Hca } \\
{[\text { ton } / \mathrm{cm}]}\end{array}$ \\
\hline 0,2009 & $-0,0228$ & $-0,0114$ \\
\hline
\end{tabular}

E para o comprimento ao longo do meridiano da casca necessário para o amortecimento das tensões de flexão causadas pela "perturbação de borda" medido a partir do vínculo

$$
-\lambda_{\text {cone }}=130,16[\mathrm{~cm}]
$$

$\mathrm{Na}$ vertical chamou-se este valor de $\lambda z$ que é igual a $\lambda_{\text {cone }} \cdot \operatorname{sen} \theta \mathrm{r}=112,72$ [cm], e portanto a tabela de valores para a casca cônica deveria ser apresentada das 
coordenadas "z" igual a $\mathrm{H}-\lambda z=926,51$ até $\mathrm{H}=1039,23$. Adotou-se de 917,99 até $1039,23[\mathrm{~cm}]$.

\subsubsection{CASCA CÔNICA COM BORDA SUPERIOR ENGASTADA}

Para os deslocamentos radiais e rotações ao longo do meridiano da superficie média da casca cônica encontrou-se

TABELA 28 - Valores dos deslocamentos radiais $\xi \mathrm{e}(\mathrm{z})$ e rotações $\phi \mathrm{e}(\mathrm{z})$

\begin{tabular}{|c|c|c|c|}
\cline { 2 - 3 } \multicolumn{1}{c|}{} & \multicolumn{2}{c|}{$\xi \mathrm{e}(\mathrm{z})$} & \multicolumn{1}{c}{$[\mathrm{cm}]$} \\
\hline $\begin{array}{c}z \\
{[\mathrm{~cm}]}\end{array}$ & Teoria & $\begin{array}{c}\text { Elementos } \\
\text { Finitos }\end{array}$ & Diferença \\
\hline 1039,23 & 0,0000 & 0,0000 & 0,0000 \\
\hline 1030,57 & 0,0315 & 0,0312 & 0,0003 \\
\hline 1021,91 & 0,0887 & 0,0891 & 0,0004 \\
\hline 1013,25 & 0,1383 & 0,1395 & 0,0012 \\
\hline 1004,59 & 0,1700 & 0,1716 & $\mathbf{0 , 0 0 1 5}$ \\
\hline 995,93 & 0,1852 & 0,1864 & 0,0012 \\
\hline 987,27 & 0,1892 & 0,1897 & 0,0005 \\
\hline 978,61 & 0,1872 & 0,1870 & 0,0003 \\
\hline 969,95 & 0,1831 & 0,1823 & 0,0008 \\
\hline 961,29 & 0,1788 & 0,1777 & 0,0011 \\
\hline 952,63 & 0,1751 & 0,1741 & 0,0011 \\
\hline 943,97 & 0,1723 & 0,1713 & 0,0010 \\
\hline 935,31 & 0,1699 & 0,1691 & 0,0008 \\
\hline 926,65 & 0,1679 & 0,1672 & 0,0007 \\
\hline 917,99 & 0,1661 & 0,1654 & 0,0006 \\
\hline
\end{tabular}

\begin{tabular}{|r|r|r|}
\hline \multicolumn{2}{|c|}{$\begin{array}{r}\text { de(z) } \\
\text { [radianos] }\end{array}$} & \multicolumn{1}{c}{} \\
\hline Teoria & $\begin{array}{c}\text { Elementos } \\
\text { Finitos }\end{array}$ & Diferença \\
\hline 0,0000 & 0,0000 & 0,0000 \\
\hline$-0,0061$ & $-0,0062$ & $\mathbf{0 , 0 0 0 1}$ \\
\hline$-0,0066$ & $-0,0067$ & 0,0001 \\
\hline$-0,0048$ & $-0,0049$ & 0,0001 \\
\hline$-0,0027$ & $-0,0027$ & 0,0000 \\
\hline$-0,0011$ & $-0,0010$ & 0,0001 \\
\hline$-0,0001$ & 0,0000 & 0,0001 \\
\hline 0,0003 & 0,0004 & 0,0001 \\
\hline 0,0004 & 0,0005 & 0,0000 \\
\hline 0,0004 & 0,0004 & 0,0000 \\
\hline 0,0003 & 0,0003 & 0,0000 \\
\hline 0,0002 & 0,0002 & 0,0000 \\
\hline 0,0002 & 0,0002 & 0,0000 \\
\hline 0,0001 & 0,0001 & 0,0000 \\
\hline 0,0001 & 0,0001 & 0,0000 \\
\hline
\end{tabular}



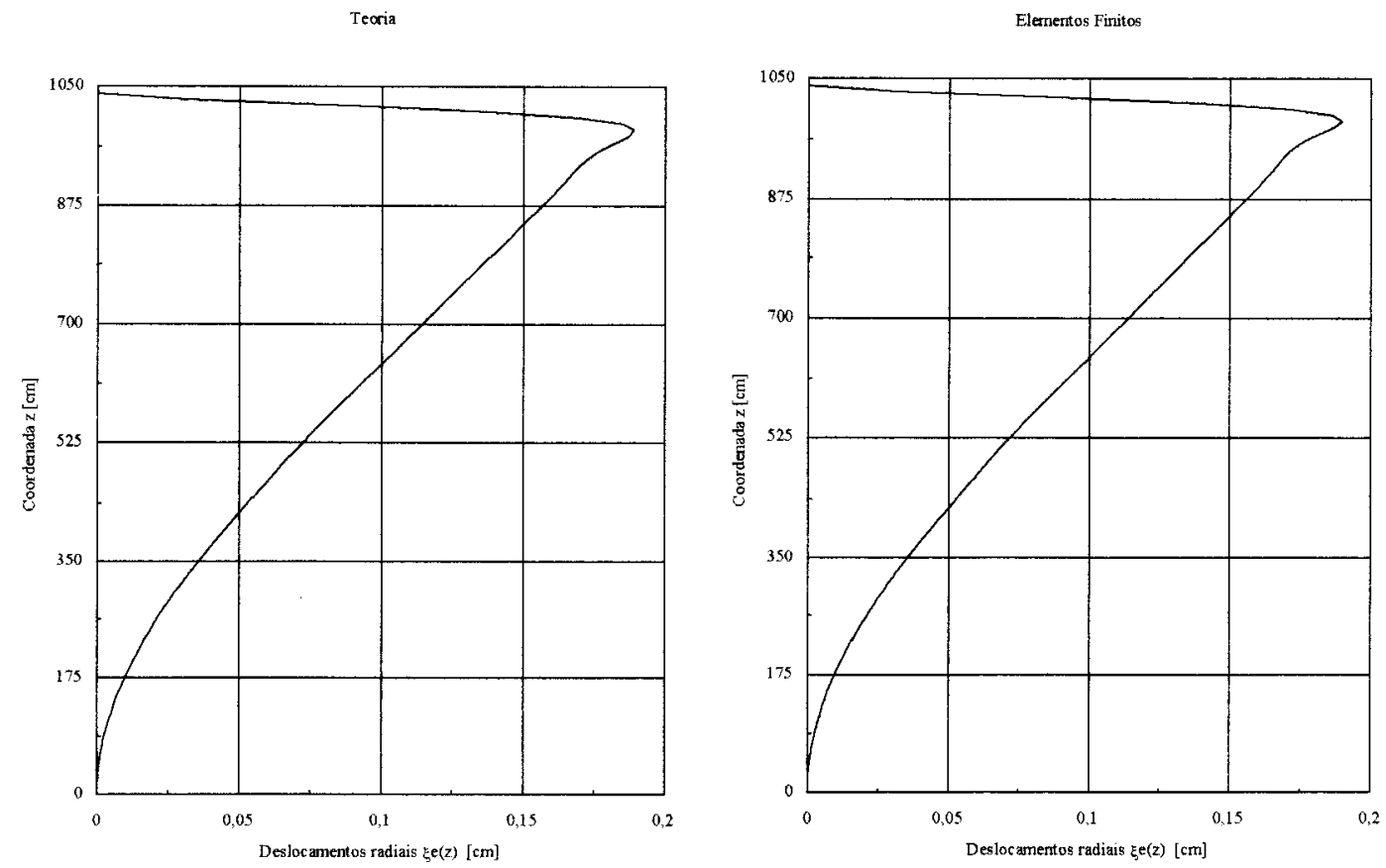

FIGURA 89 - Gráficos para os valores dos deslocamentos radiais $\xi_{\mathrm{e}}(\mathrm{z})$ longo do meridiano da superfície média da casca cônica
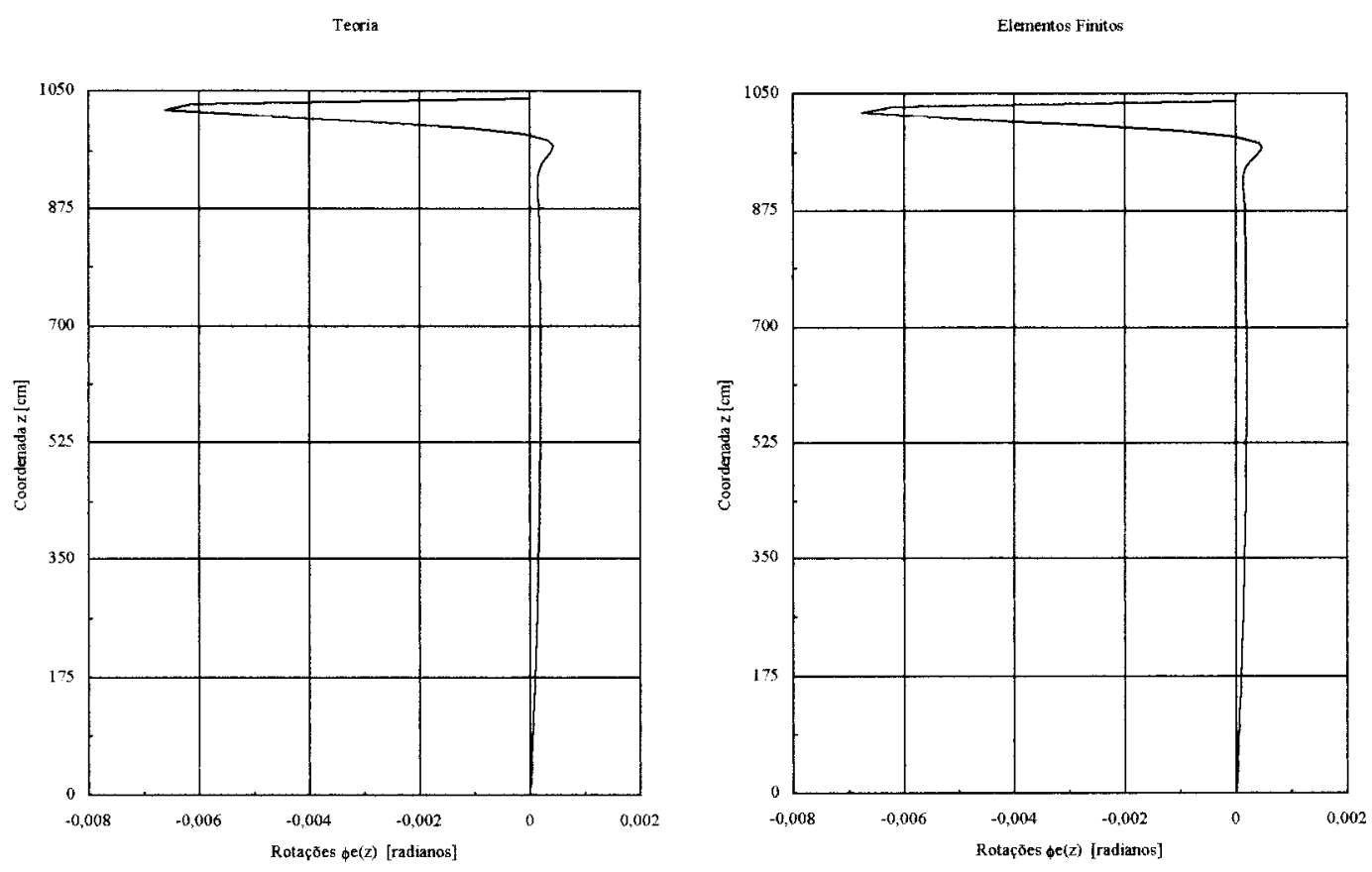

FIGURA 90 - Gráficos para os valores das rotações $\phi \mathrm{e}(\mathrm{z})$ ao longo do meridiano da superficie média da casca cônica 
Lembrando que a coluna "Diferença", da tabela, apresenta os valores das diferenças absolutas entre os parâmetros obtidos pelas equações da teoria aproximada e os obtidos pelo cálculo através do método dos elementos finitos.

Os momentos solicitantes ao longo dos paralelos e dos meridianos da superficie média da casca cônica e suas correspondentes diferenças absolutas foram :

TABELA 29 - Valores dos momentos ao longo dos paralelos $\mathrm{M} 1 \mathrm{e}(\mathrm{z})$ e dos meridianos M2e(z)

\begin{tabular}{|c|r|r|r|}
\cline { 2 - 3 } \multicolumn{1}{c|}{} & \multicolumn{2}{c|}{\begin{tabular}{c}
\multicolumn{1}{c}{ M1e(z) } \\
[ton.cm/cm]
\end{tabular}} & \multicolumn{1}{c}{} \\
\hline $\begin{array}{c}\mathrm{z} \\
{[\mathrm{cm}]}\end{array}$ & Teoria & $\begin{array}{c}\text { Elementos } \\
\text { Finitos }\end{array}$ & Diferença \\
\hline 1039,23 & 0,2009 & 0,1908 & $\mathbf{0 , 0 1 0 1}$ \\
\hline 1030,57 & 0,0504 & 0,0459 & 0,0045 \\
\hline 1021,91 & $-0,0210$ & $-0,0252$ & 0,0042 \\
\hline 1013,25 & $-0,0417$ & $-0,0455$ & 0,0038 \\
\hline 1004,59 & $-0,0373$ & $-0,0398$ & 0,0025 \\
\hline 995,93 & $-0,0247$ & $-0,0256$ & 0,0009 \\
\hline 987,27 & $-0,0128$ & $-0,0125$ & 0,0003 \\
\hline 978,61 & $-0,0045$ & $-0,0037$ & 0,0008 \\
\hline 969,95 & $-0,0001$ & 0,0008 & 0,0009 \\
\hline 961,29 & 0,0016 & 0,0022 & 0,0006 \\
\hline 952,63 & 0,0018 & 0,0021 & 0,0003 \\
\hline 943,97 & 0,0013 & 0,0013 & 0,0000 \\
\hline 935,31 & 0,0008 & 0,0006 & 0,0002 \\
\hline 926,65 & 0,0003 & 0,0002 & 0,0001 \\
\hline 917,99 & 0,0001 & $-0,0001$ & 0,0002 \\
\hline
\end{tabular}

\begin{tabular}{|r|r|r|}
\hline \multicolumn{2}{|c|}{$\begin{array}{c}\text { M2e(z) } \\
\text { [ton.cm/cm] }\end{array}$} & \\
\hline Teoria & $\begin{array}{c}\text { Elementos } \\
\text { Finitos }\end{array}$ & Diferença \\
\hline 0,0602 & 0,0573 & $\mathbf{0 , 0 0 3 0}$ \\
\hline 0,0141 & 0,0129 & 0,0013 \\
\hline$-0,0074$ & $-0,0086$ & 0,0012 \\
\hline$-0,0133$ & $-0,0144$ & 0,0011 \\
\hline$-0,0117$ & $-0,0123$ & 0,0007 \\
\hline$-0,0076$ & $-0,0078$ & 0,0002 \\
\hline$-0,0039$ & $-0,0038$ & 0,0001 \\
\hline$-0,0013$ & $-0,0010$ & 0,0003 \\
\hline 0,0000 & 0,0003 & 0,0003 \\
\hline 0,0005 & 0,0007 & 0,0002 \\
\hline 0,0006 & 0,0007 & 0,0001 \\
\hline 0,0004 & 0,0004 & 0,0000 \\
\hline 0,0002 & 0,0002 & 0,0000 \\
\hline 0,0001 & 0,0001 & 0,0000 \\
\hline 0,0000 & 0,0000 & 0,0000 \\
\hline
\end{tabular}



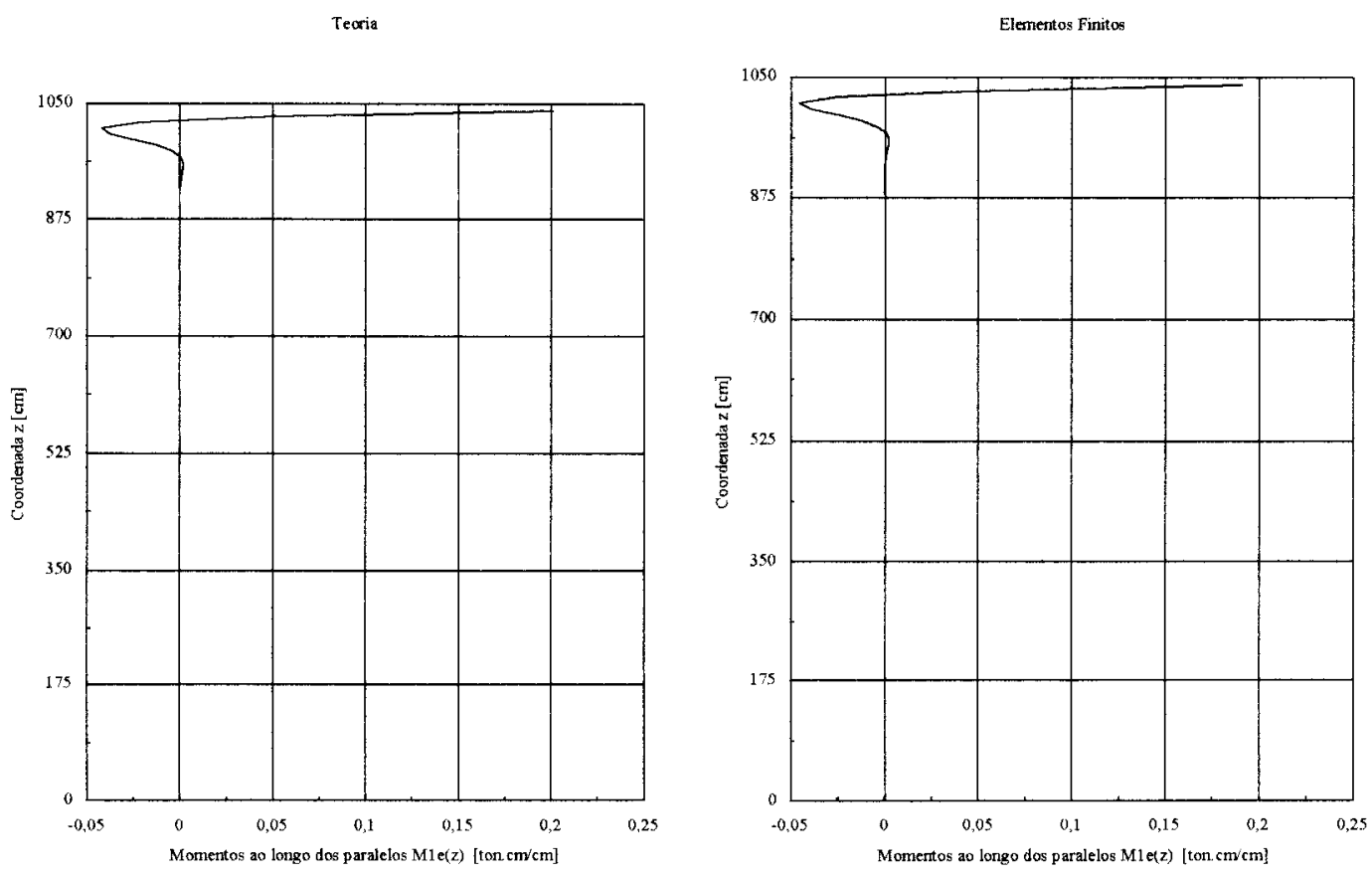

FIGURA 91 - Gráficos para os valores dos momentos ao longo dos paralelos M1e(z) da superfície média da casca cônica

Tearia

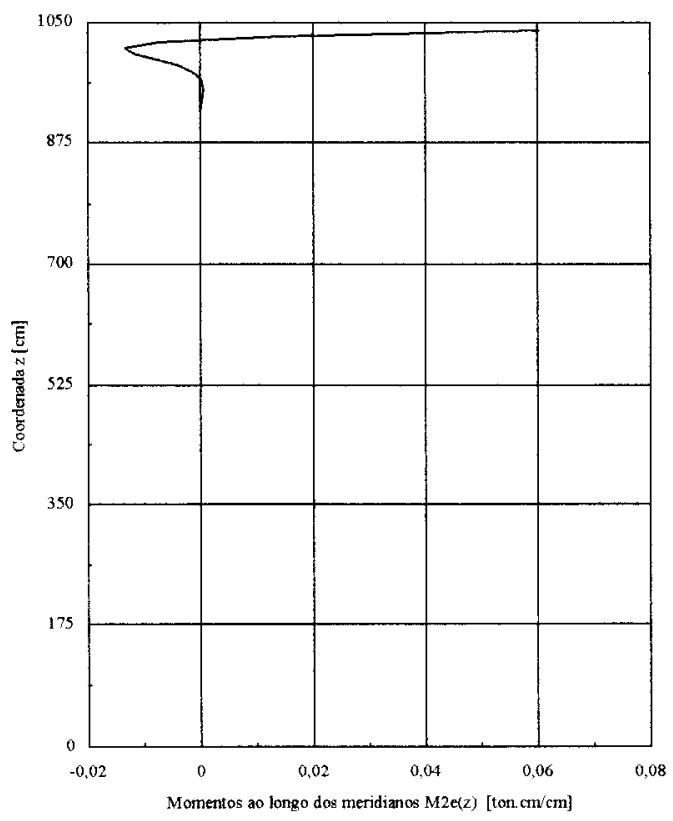

Elementos Finitos

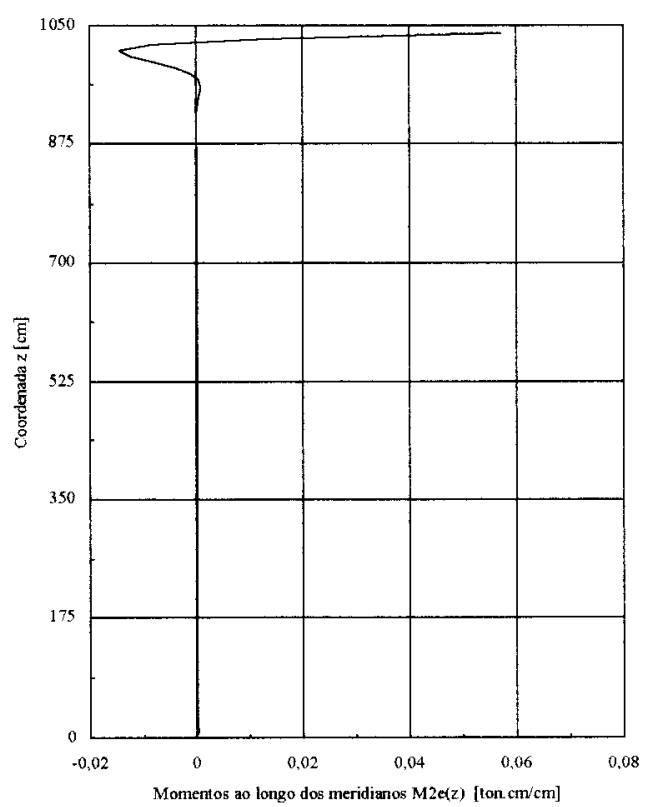

FIGURA 92 - Gráficos para os valores dos momentos ao longo dos meridianos $\mathrm{M} 2 \mathrm{e}(\mathrm{z})$ da superfície média da casca cônica 
Para as tensões atuantes ao longo dos meridianos da superficie interna e externa da casca cônica obteve-se :

TABELA 30 - Valores para as tensões ao longo dos meridianos da superficie interna $\sigma$ lein(z) e externa $\sigma$ leex(z) da casca

\begin{tabular}{|c|r|r|r|}
\cline { 2 - 3 } \multicolumn{1}{c|}{} & \multicolumn{2}{c|}{\begin{tabular}{c}
\multicolumn{1}{c}{$\sigma$ ein(z) } \\
{$\left[\right.$ ton/ $\left.\mathrm{cm}^{2}\right]$}
\end{tabular}} & \multicolumn{1}{c}{} \\
\hline $\begin{array}{c}z \\
{[\mathrm{~cm}]}\end{array}$ & Teoria & $\begin{array}{c}\text { Elementos } \\
\text { Finitos }\end{array}$ & Diferença \\
\hline 1039,23 & $-0,6808$ & $-0,6391$ & $\mathbf{0 , 0 4 1 8}$ \\
\hline 1030,57 & 0,2246 & 0,2418 & 0,0173 \\
\hline 1021,91 & 0,6543 & 0,6796 & 0,0254 \\
\hline 1013,25 & 0,7783 & 0,8038 & 0,0255 \\
\hline 1004,59 & 0,7502 & 0,7677 & 0,0176 \\
\hline 995,93 & 0,6722 & 0,6791 & 0,0069 \\
\hline 987,27 & 0,5975 & 0,5958 & 0,0017 \\
\hline 978,61 & 0,5451 & 0,5388 & 0,0063 \\
\hline 969,95 & 0,5156 & 0,5084 & 0,0072 \\
\hline 961,29 & 0,5026 & 0,4966 & 0,0059 \\
\hline 952,63 & 0,4987 & 0,4948 & 0,0040 \\
\hline 943,97 & 0,4986 & 0,4964 & 0,0023 \\
\hline 935,31 & 0,4991 & 0,4979 & 0,0013 \\
\hline 926,65 & 0,4989 & 0,4980 & 0,0009 \\
\hline 917,99 & 0,4975 & 0,4966 & 0,0009 \\
\hline
\end{tabular}

\begin{tabular}{|r|r|r|}
\hline \multicolumn{2}{|c|}{$\begin{array}{c}\sigma l e e x(z) \\
{\left[\text { ton/cm }{ }^{2}\right]}\end{array}$} & \multicolumn{1}{|c|}{} \\
\cline { 1 - 2 } Teoria & $\begin{array}{c}\text { Elementos } \\
\text { Finitos }\end{array}$ & Diferença \\
\hline 1,7294 & 1,6510 & $\mathbf{0 , 0 7 8 4}$ \\
\hline 0,8295 & 0,7925 & 0,0371 \\
\hline 0,4024 & 0,3767 & 0,0257 \\
\hline 0,2775 & 0,2584 & 0,0191 \\
\hline 0,3022 & 0,2907 & 0,0115 \\
\hline 0,3753 & 0,3714 & 0,0039 \\
\hline 0,4443 & 0,4458 & 0,0015 \\
\hline 0,4909 & 0,4946 & 0,0037 \\
\hline 0,5146 & 0,5179 & 0,0033 \\
\hline 0,5218 & 0,5234 & 0,0016 \\
\hline 0,5200 & 0,5196 & 0,0004 \\
\hline 0,5144 & 0,5126 & 0,0019 \\
\hline 0,5083 & 0,5056 & 0,0027 \\
\hline 0,5028 & 0,4999 & 0,0029 \\
\hline 0,4983 & 0,4956 & 0,0027 \\
\hline
\end{tabular}


Tearia

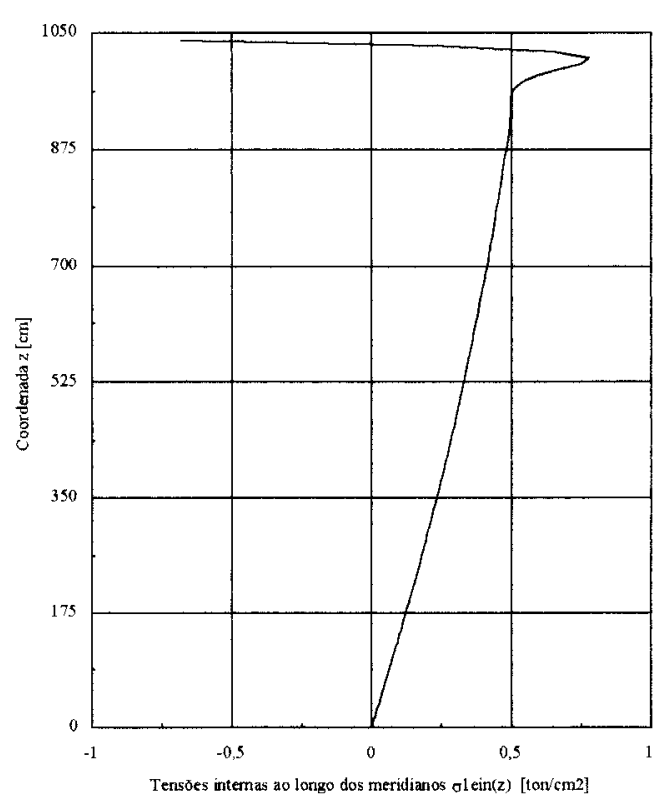

Elementos Finitos

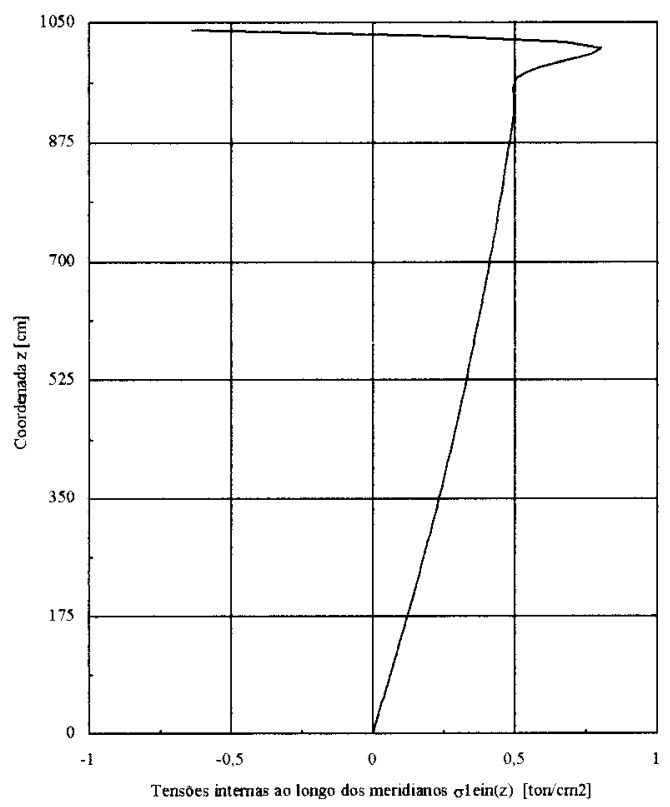

FIGURA 93 - Gráficos para os valores das tensões ao longo dos meridianos da superfície interna $\sigma$ lein(z) da casca cônica

Tearia

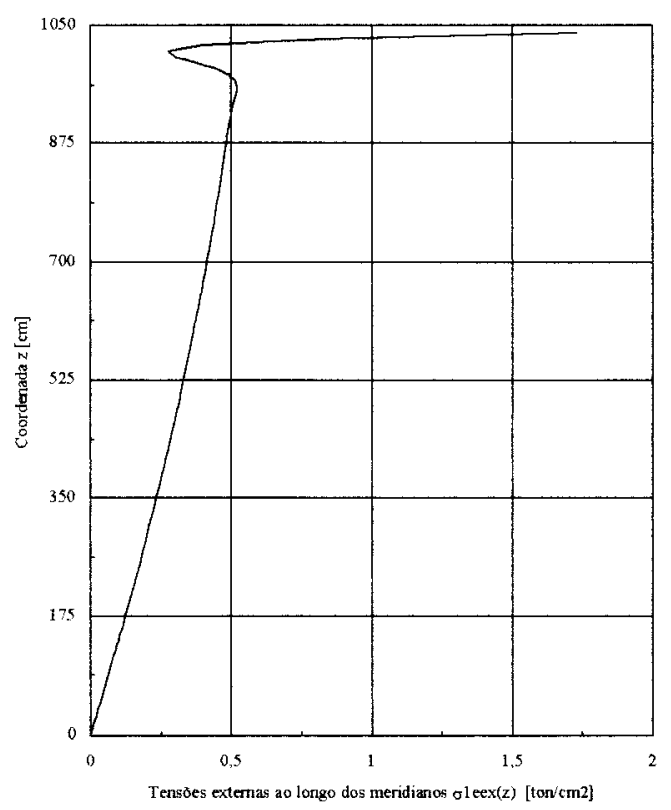

Elemeritos Finitos

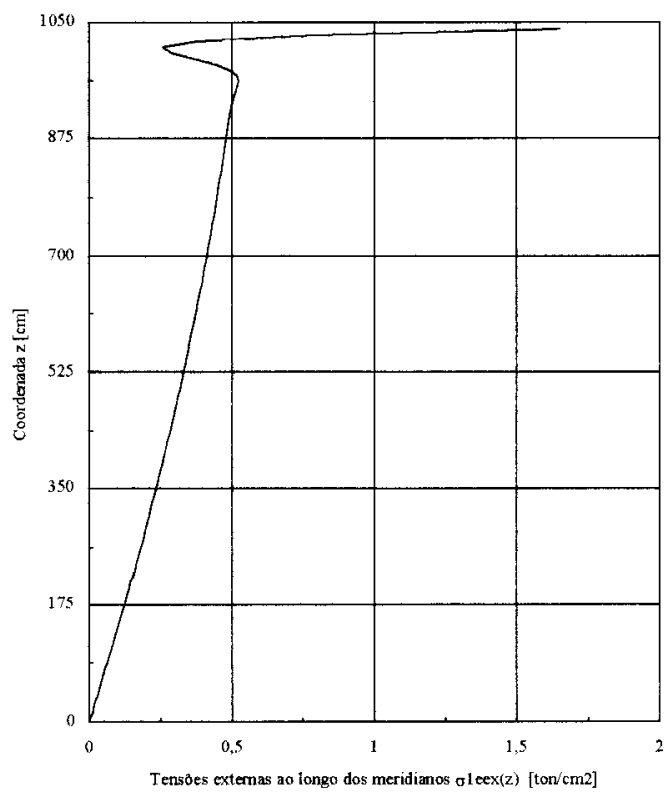

FIGURA 94 - Gráficos para os valores das tensões ao longo dos meridianos da superficie externa $\sigma$ leex(z) da casca cônica 
E finalmente para as tensões atuantes ao longo dos paralelos da superficie interna e externa da casca cônica encontrou-se

TABELA 31 - Valores para as tensões ao longo dos paralelos da superfície interna $\sigma 2$ ein(z) e externa $\sigma 2 \operatorname{eex}(z)$ da casca

\begin{tabular}{|c|r|r|r|}
\cline { 2 - 3 } \multicolumn{1}{c|}{} & \multicolumn{2}{c|}{$\begin{array}{c}\sigma 2 \mathrm{ein}(\mathrm{z}) \\
{\left[\text { ton/ } \mathrm{cm}^{2}\right]}\end{array}$} & \multicolumn{1}{c}{} \\
\hline $\begin{array}{c}z \\
{[\mathrm{~cm}]}\end{array}$ & Teoria & $\begin{array}{c}\text { Elementos } \\
\text { Finitos }\end{array}$ & Diferença \\
\hline 1039,23 & $-0,2007$ & $-0,1917$ & $\mathbf{0 , 0 0 9 0}$ \\
\hline 1030,57 & 0,1911 & 0,1883 & 0,0028 \\
\hline 1021,91 & 0,5251 & 0,5269 & 0,0018 \\
\hline 1013,25 & 0,7392 & 0,7464 & 0,0072 \\
\hline 1004,59 & 0,8450 & 0,8540 & 0,0090 \\
\hline 995,93 & 0,8786 & 0,8855 & 0,0069 \\
\hline 987,27 & 0,8748 & 0,8775 & 0,0028 \\
\hline 978,61 & 0,8575 & 0,8562 & 0,0012 \\
\hline 969,95 & 0,8396 & 0,8357 & 0,0039 \\
\hline 961,29 & 0,8262 & 0,8211 & 0,0051 \\
\hline 952,63 & 0,8178 & 0,8127 & 0,0051 \\
\hline 943,97 & 0,8132 & 0,8087 & 0,0045 \\
\hline 935,31 & 0,8107 & 0,8068 & 0,0039 \\
\hline 926,65 & 0,8090 & 0,8057 & 0,0034 \\
\hline 917,99 & 0,8075 & 0,8044 & 0,0031 \\
\hline
\end{tabular}

\begin{tabular}{|c|c|c|}
\hline \multicolumn{2}{|c|}{$\begin{array}{l}\sigma 2 \operatorname{eex}(\mathrm{z}) \\
{\left[\mathrm{ton} / \mathrm{cm}^{2}\right]}\end{array}$} & \\
\hline Teoria & $\begin{array}{c}\text { Elementos } \\
\text { Finitos }\end{array}$ & Diferença \\
\hline 0,5221 & 0,4953 & 0,0268 \\
\hline 0,3606 & 0,3426 & 0,0180 \\
\hline 0,4366 & 0,4240 & 0,0126 \\
\hline 0,5794 & 0,5740 & 0,0054 \\
\hline 0,7052 & 0,7060 & 0,0008 \\
\hline 0,7872 & 0,7913 & 0,0041 \\
\hline 0,8284 & 0,8325 & 0,0041 \\
\hline 0,8416 & 0,8437 & 0,0021 \\
\hline 0,8399 & 0,8394 & 0,0004 \\
\hline 0,8325 & 0,8299 & 0,0026 \\
\hline 0,8245 & 0,8208 & 0,0038 \\
\hline 0,8181 & 0,8139 & 0,0042 \\
\hline 0,8135 & 0,8094 & 0,0041 \\
\hline 0,8102 & 0,8065 & 0,0037 \\
\hline 0,8077 & 0,8043 & 0,0034 \\
\hline
\end{tabular}



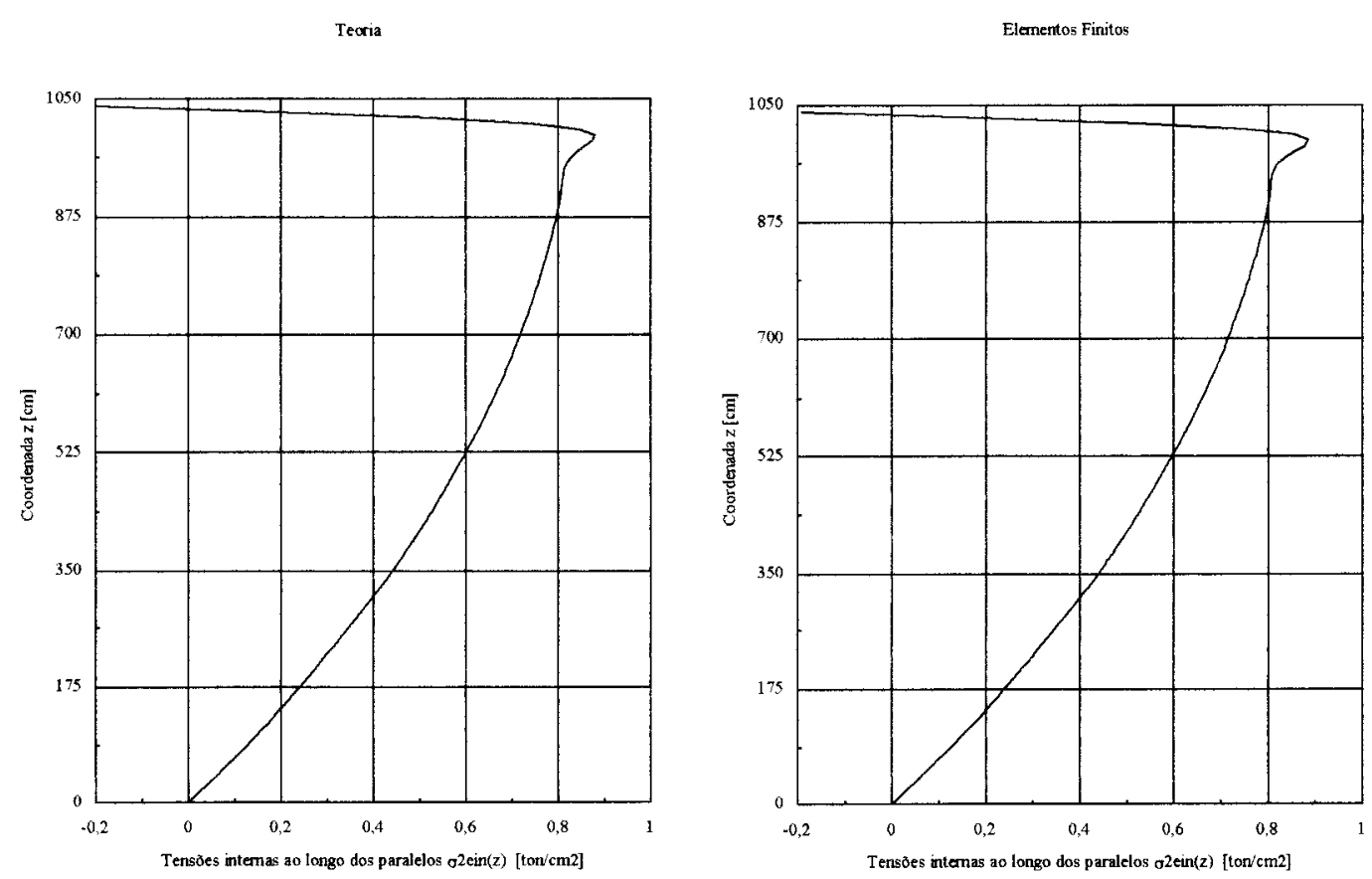

FIGURA 95 - Gráficos para os valores das tensões ao longo dos paralelos da superficie interna $\sigma 2 \operatorname{ein}(\mathrm{z})$ da casca cônica
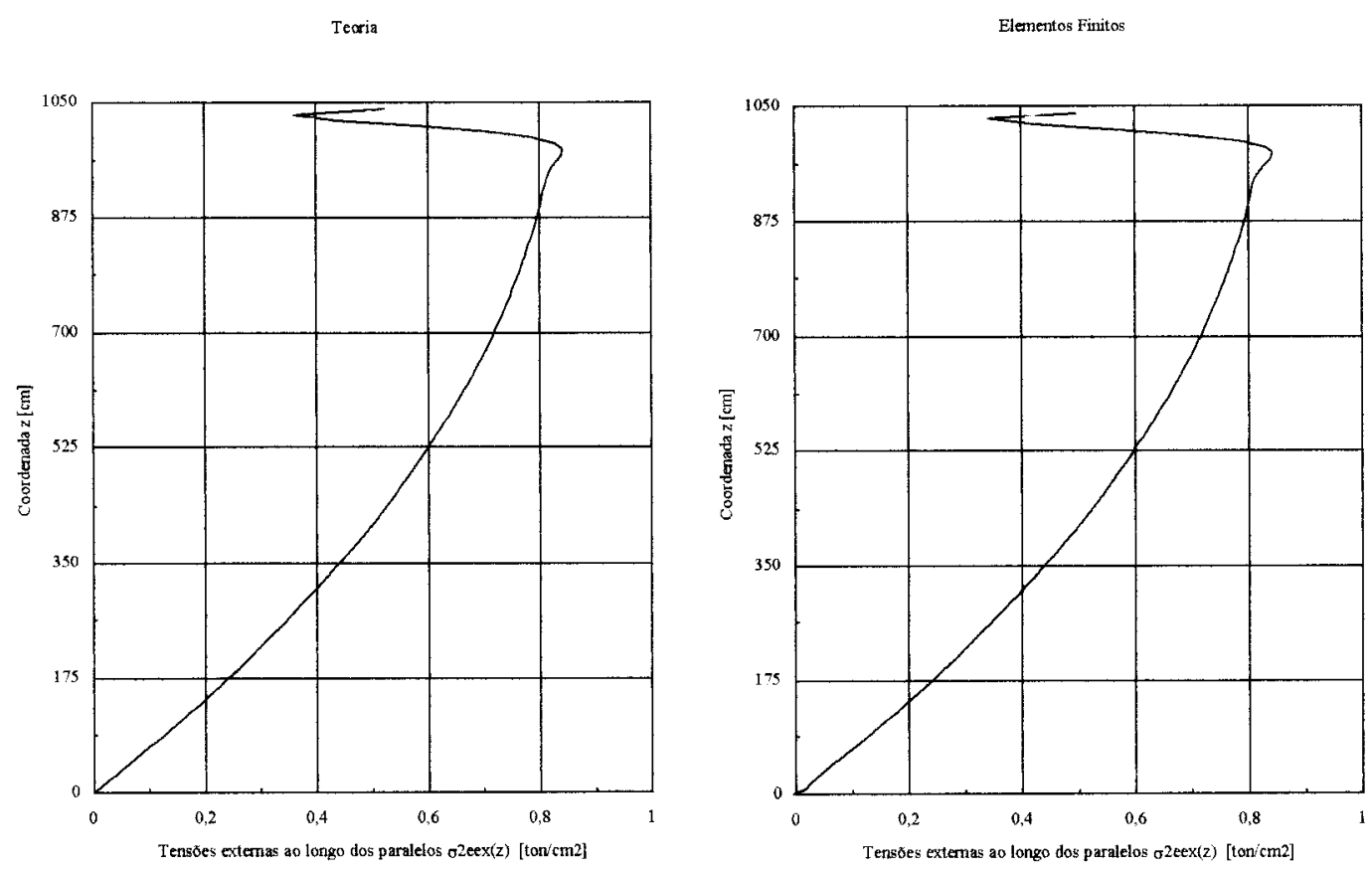

FIGURA 96 - Gráficos para os valores das tensões ao longo dos paralelos da superficie externa $\sigma 2 \operatorname{eex}(\mathrm{z})$ da casca cônica 
São apresentados abaixo os valores encontrados para as tensões de membrana atuantes ao longo dos meridianos e dos paralelos da superfície média da casca cônica para comparação :

TABELA 32 - Valores para as tensões teóricas de membrana ao longo dos meridianos $\sigma 1 \mathrm{~m}(\mathrm{z})$ e paralelos $\sigma 2 \mathrm{~m}(\mathrm{z})$ da superficie média da casca

\begin{tabular}{|c|r|r|}
\hline $\begin{array}{c}\mathrm{z} \\
{[\mathrm{cm}]}\end{array}$ & $\begin{array}{c}\sigma 1 \mathrm{~m}(\mathrm{z}) \\
{\left[\text { ton } / \mathrm{cm}^{2}\right]}\end{array}$ & $\begin{array}{c}\sigma 2 \mathrm{~m}(\mathrm{z}) \\
{\left[\text { ton } / \mathrm{cm}^{2}\right]}\end{array}$ \\
\hline 1039,23 & 0,5357 & 0,8314 \\
\hline 1030,57 & 0,5332 & 0,8304 \\
\hline 1021,91 & 0,5307 & 0,8293 \\
\hline 1013,25 & 0,5281 & 0,8281 \\
\hline 1004,59 & 0,5256 & 0,8269 \\
\hline 995,93 & 0,5230 & 0,8255 \\
\hline 987,27 & 0,5203 & 0,8240 \\
\hline 978,61 & 0,5176 & 0,8224 \\
\hline 969,95 & 0,5149 & 0,8208 \\
\hline 961,29 & 0,5122 & 0,8190 \\
\hline 952,63 & 0,5094 & 0,8171 \\
\hline 943,97 & 0,5066 & 0,8151 \\
\hline 935,31 & 0,5037 & 0,8130 \\
\hline 926,65 & 0,5008 & 0,8109 \\
\hline 917,99 & 0,4979 & 0,8086 \\
\hline
\end{tabular}

Pode-se ver abaixo nos gráficos com as tensões teóricas ao longo dos meridianos e paralelos da casca cônica, que as mesmas possuem seus valores praticamente coincidêntes abaixo da região de $z=H-\lambda z=926,51[\mathrm{~cm}]$ 
Teoria

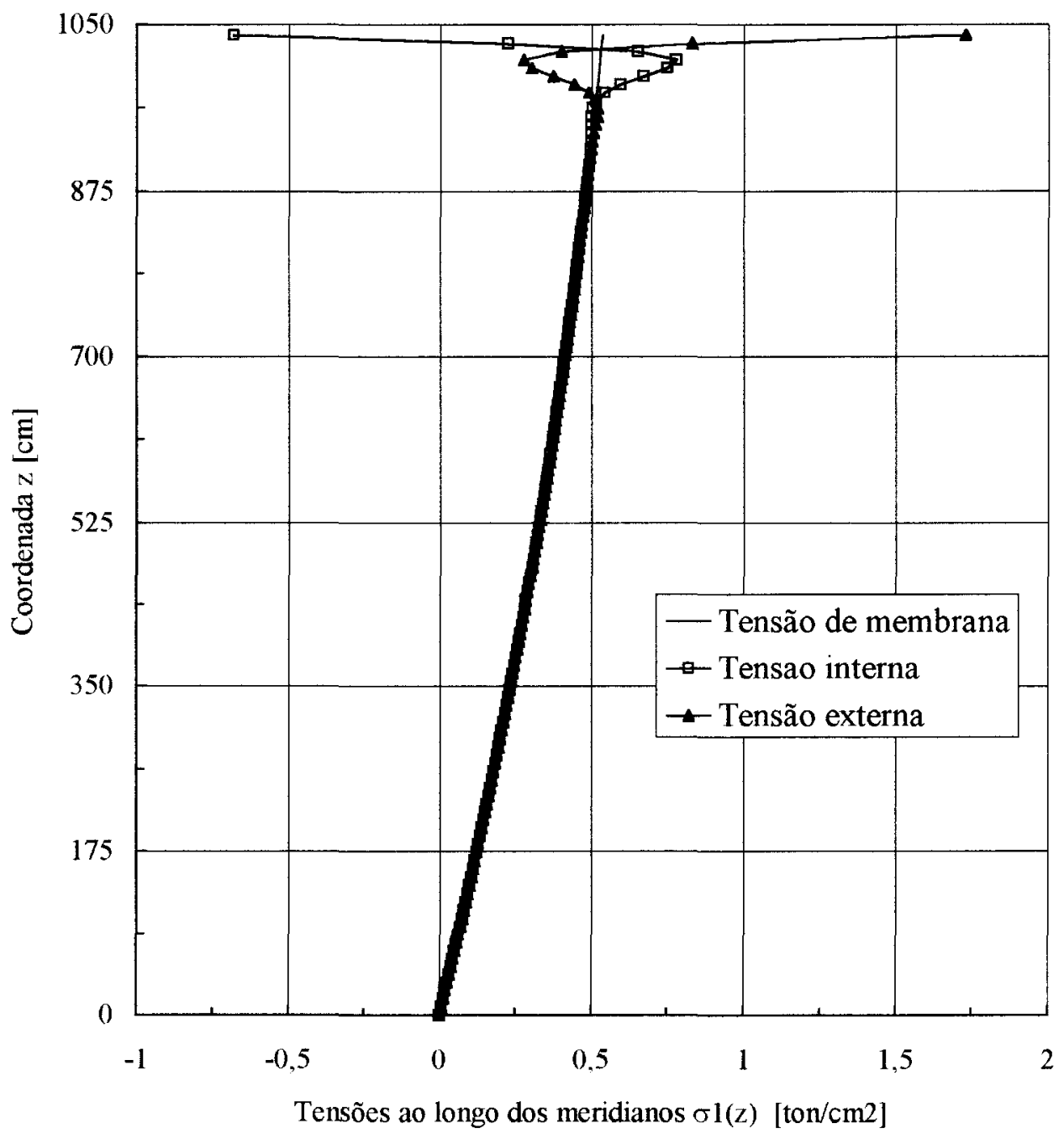

FIGURA 97 - Gráfico comparativo para os valores das tensões ao longo dos meridianos da casca cônica : $\sigma 1 \mathrm{~m}(\mathrm{z}), \sigma \operatorname{lein}(\mathrm{z})$ e $\sigma \operatorname{leex}(\mathrm{z})$ 
Teoria

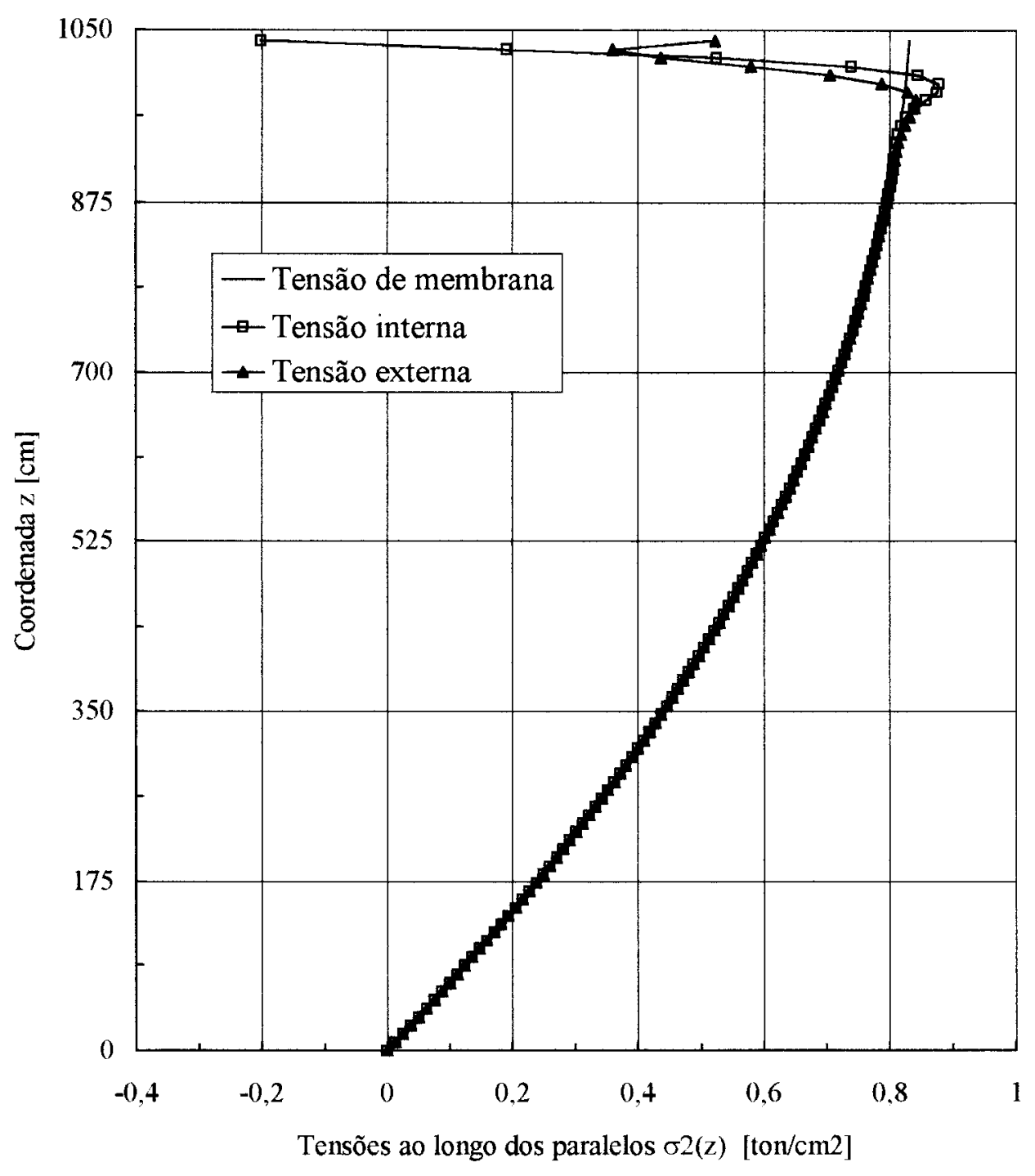

FIGURA 98 - Gráfico comparativo para os valores das tensões ao longo dos paralelos da casca cônica : $\sigma 2 \mathrm{~m}(\mathrm{z}), \sigma 2 \operatorname{ein}(\mathrm{z})$ e $\sigma 2 \operatorname{eex}(\mathrm{z})$ 


\subsubsection{CASCA CÔNICA COM BORDA SUPERIOR APOIADA FIXA}

Para os deslocamentos radiais e rotações ao longo do meridiano da superfície média da casca cônica encontrou-se

TABELA 33 - Valores dos deslocamentos radiais $\xi \mathrm{a}(\mathrm{z})$ e rotações $\phi \mathrm{a}(\mathrm{z})$

\begin{tabular}{|c|c|c|c|}
\cline { 2 - 3 } \multicolumn{1}{c|}{} & \multicolumn{2}{c|}{$\xi \mathrm{a}(\mathrm{z})$} \\
\hline $\begin{array}{c}\mathrm{cm}] \\
{[\mathrm{cm}]}\end{array}$ & Teoria & $\begin{array}{c}\text { Elementos } \\
\text { Finitos }\end{array}$ & Diferença \\
\hline 1039,23 & 0,0000 & 0,0000 & 0,0000 \\
\hline 1030,57 & 0,0861 & 0,0851 & 0,0010 \\
\hline 1021,91 & 0,1479 & 0,1476 & 0,0002 \\
\hline 1013,25 & 0,1819 & 0,1824 & 0,0005 \\
\hline 1004,59 & 0,1950 & 0,1956 & 0,0006 \\
\hline 995,93 & 0,1958 & 0,1960 & 0,0002 \\
\hline 987,27 & 0,1913 & 0,1909 & 0,0004 \\
\hline 978,61 & 0,1855 & 0,1847 & 0,0009 \\
\hline 969,95 & 0,1805 & 0,1794 & $\mathbf{0 , 0 0 1 1}$ \\
\hline 961,29 & 0,1766 & 0,1755 & 0,0011 \\
\hline 952,63 & 0,1737 & 0,1728 & 0,0010 \\
\hline 943,97 & 0,1716 & 0,1707 & 0,0008 \\
\hline 935,31 & 0,1697 & 0,1690 & 0,0007 \\
\hline 926,65 & 0,1680 & 0,1673 & 0,0006 \\
\hline 917,99 & 0,1662 & 0,1656 & 0,0006 \\
\hline
\end{tabular}

\begin{tabular}{|c|c|c|}
\hline \multicolumn{2}{|c|}{$\begin{array}{c}\phi \mathrm{a}(\mathrm{z}) \\
\text { [radianos] }\end{array}$} & \multirow[b]{2}{*}{ Diferença } \\
\hline Teoria & $\begin{array}{c}\text { Elementos } \\
\text { Finitos } \\
\end{array}$ & \\
\hline$-0,0107$ & $-0,0104$ & 0,0002 \\
\hline$-0,0089$ & $-0,0089$ & 0,0001 \\
\hline$-0,0055$ & $-0,0057$ & 0,0001 \\
\hline$-0,0026$ & $-0,0027$ & 0,0001 \\
\hline$-0,0007$ & $-0,0007$ & 0,0000 \\
\hline 0,0002 & 0,0003 & 0,0001 \\
\hline 0,0006 & 0,0006 & 0,0001 \\
\hline 0,0006 & 0,0006 & 0,0000 \\
\hline 0,0004 & 0,0005 & 0,0000 \\
\hline 0,0003 & 0,0003 & 0,0000 \\
\hline 0,0002 & 0,0002 & 0,0000 \\
\hline 0,0002 & 0,0001 & 0,0000 \\
\hline 0,0001 & 0,0001 & 0,0000 \\
\hline 0,0001 & 0,0001 & 0,0000 \\
\hline 0,0001 & 0,0001 & 0,0000 \\
\hline
\end{tabular}



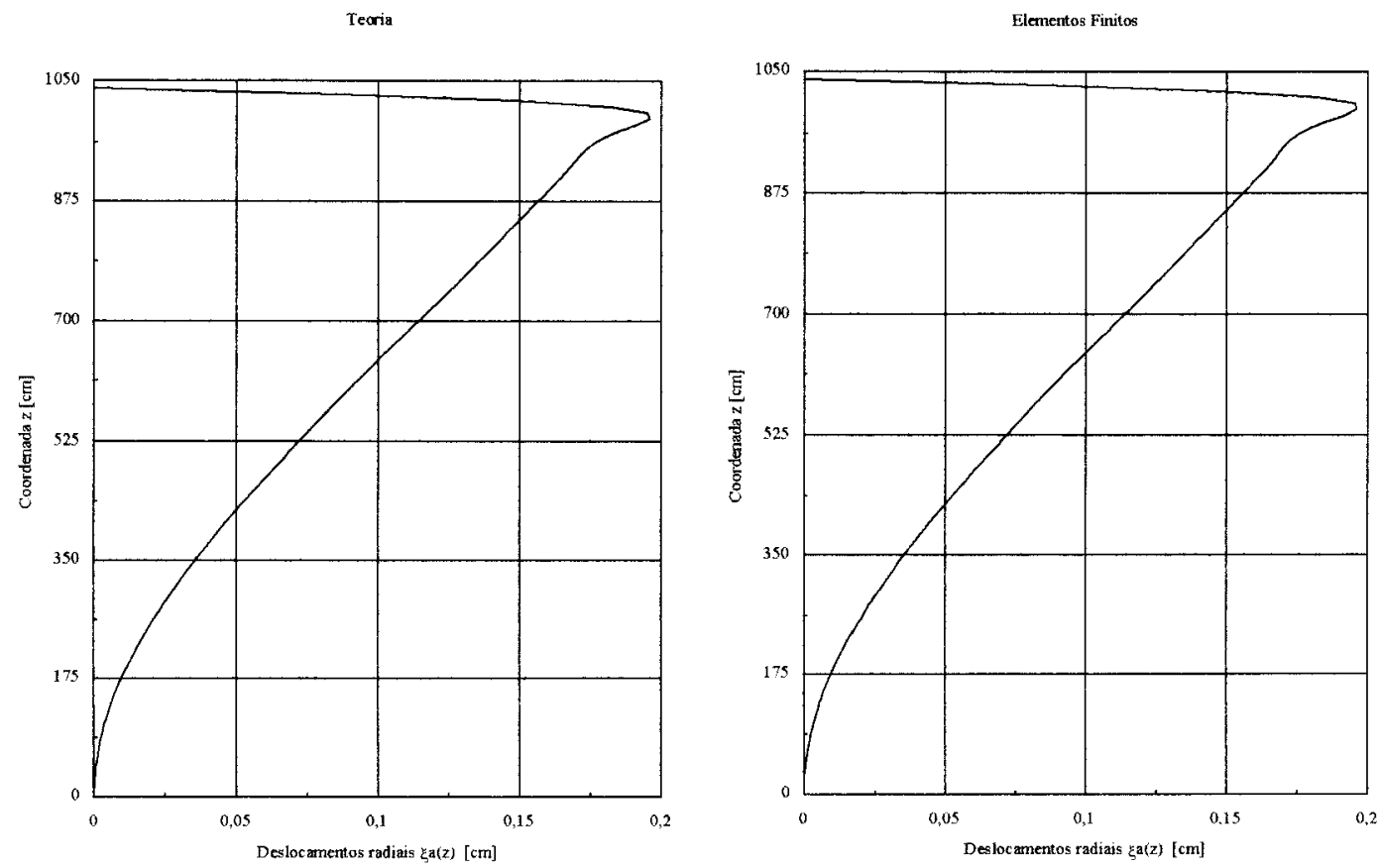

FIGURA 99 - Gráficos para os valores dos deslocamentos radiais $\xi_{\mathrm{a}}(\mathrm{z})$ longo do meridiano da superfície média da casca cônica
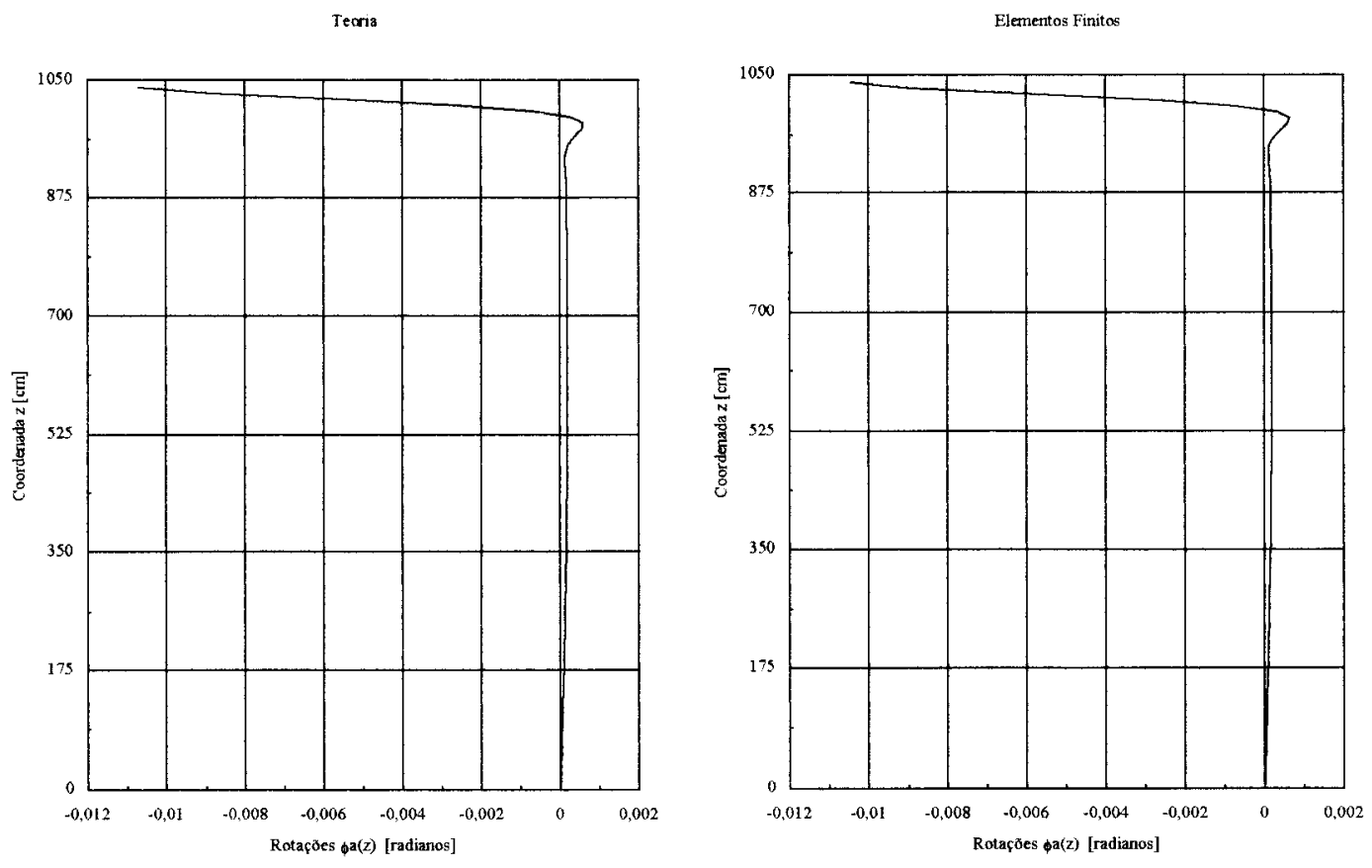

FIGURA 100 - Gráficos para os valores das rotações $\phi \mathrm{a}(\mathrm{z})$ ao longo do meridiano da superficie média da casca cônica 
Os momentos solicitantes ao longo dos paralelos e dos meridianos da superfície média da casca cônica e suas correspondentes diferenças absolutas foram :

TABELA 34 - Valores dos momentos ao longo dos paralelos

Mla(z) e dos meridianos M2a(z)

\begin{tabular}{|c|c|c|c|}
\hline & \multicolumn{2}{|c|}{$\begin{array}{c}\mathrm{Mla}(\mathrm{z}) \\
{[\text { ton } \mathrm{cm} / \mathrm{cm}]}\end{array}$} & \multirow[b]{2}{*}{ Diferença } \\
\hline $\begin{array}{c}\mathrm{z} \\
{[\mathrm{cm}]}\end{array}$ & Teoria & $\begin{array}{c}\text { Elementos } \\
\text { Finitos }\end{array}$ & \\
\hline 1039,23 & 0,0000 & $-0,0004$ & 0,0004 \\
\hline 1030,57 & $-0,0584$ & $-0,0598$ & 0,0014 \\
\hline 1021,91 & $-0,0633$ & $-0,0658$ & 0,0025 \\
\hline 1013,25 & $-0,0466$ & $-0,0487$ & 0,0021 \\
\hline 1004,59 & $-0,0267$ & $-0,0276$ & 0,0009 \\
\hline 995,93 & $-0,0114$ & $-0,0112$ & 0,0002 \\
\hline 987,27 & $-0,0023$ & $-0,0015$ & 0,0008 \\
\hline 978,61 & 0,0018 & 0,0026 & 0,0008 \\
\hline 969,95 & 0,0028 & 0,0034 & 0,0006 \\
\hline 961,29 & 0,0024 & 0,0026 & 0,0002 \\
\hline 952,63 & 0,0015 & 0,0015 & 0,0000 \\
\hline 943,97 & 0,0007 & 0,0006 & 0,0001 \\
\hline 935,31 & 0,0002 & 0,0001 & 0,0002 \\
\hline 926,65 & 0,0000 & $-0,0002$ & 0,0002 \\
\hline 917,99 & $-0,0001$ & $-0,0002$ & 0,0001 \\
\hline
\end{tabular}

\begin{tabular}{|r|r|r|}
\hline \multicolumn{2}{|c|}{$\begin{array}{c}\text { M2a(z) } \\
\text { [ton.cm/cm] }\end{array}$} & \\
\hline Teoria & $\begin{array}{c}\text { Elementos } \\
\text { Finitos }\end{array}$ & Diferença \\
\hline$-0,0017$ & $-0,0017$ & 0,0001 \\
\hline$-0,0190$ & $-0,0192$ & 0,0003 \\
\hline$-0,0199$ & $-0,0206$ & $\mathbf{0 , 0 0 0 7}$ \\
\hline$-0,0144$ & $-0,0150$ & 0,0006 \\
\hline$-0,0081$ & $-0,0084$ & 0,0002 \\
\hline$-0,0034$ & $-0,0033$ & 0,0001 \\
\hline$-0,0006$ & $-0,0004$ & 0,0003 \\
\hline 0,0006 & 0,0009 & 0,0003 \\
\hline 0,0009 & 0,0011 & 0,0002 \\
\hline 0,0007 & 0,0008 & 0,0001 \\
\hline 0,0005 & 0,0005 & 0,0000 \\
\hline 0,0002 & 0,0002 & 0,0000 \\
\hline 0,0001 & 0,0000 & 0,0000 \\
\hline 0,0000 & 0,0000 & 0,0000 \\
\hline 0,0000 & 0,0000 & 0,0000 \\
\hline
\end{tabular}



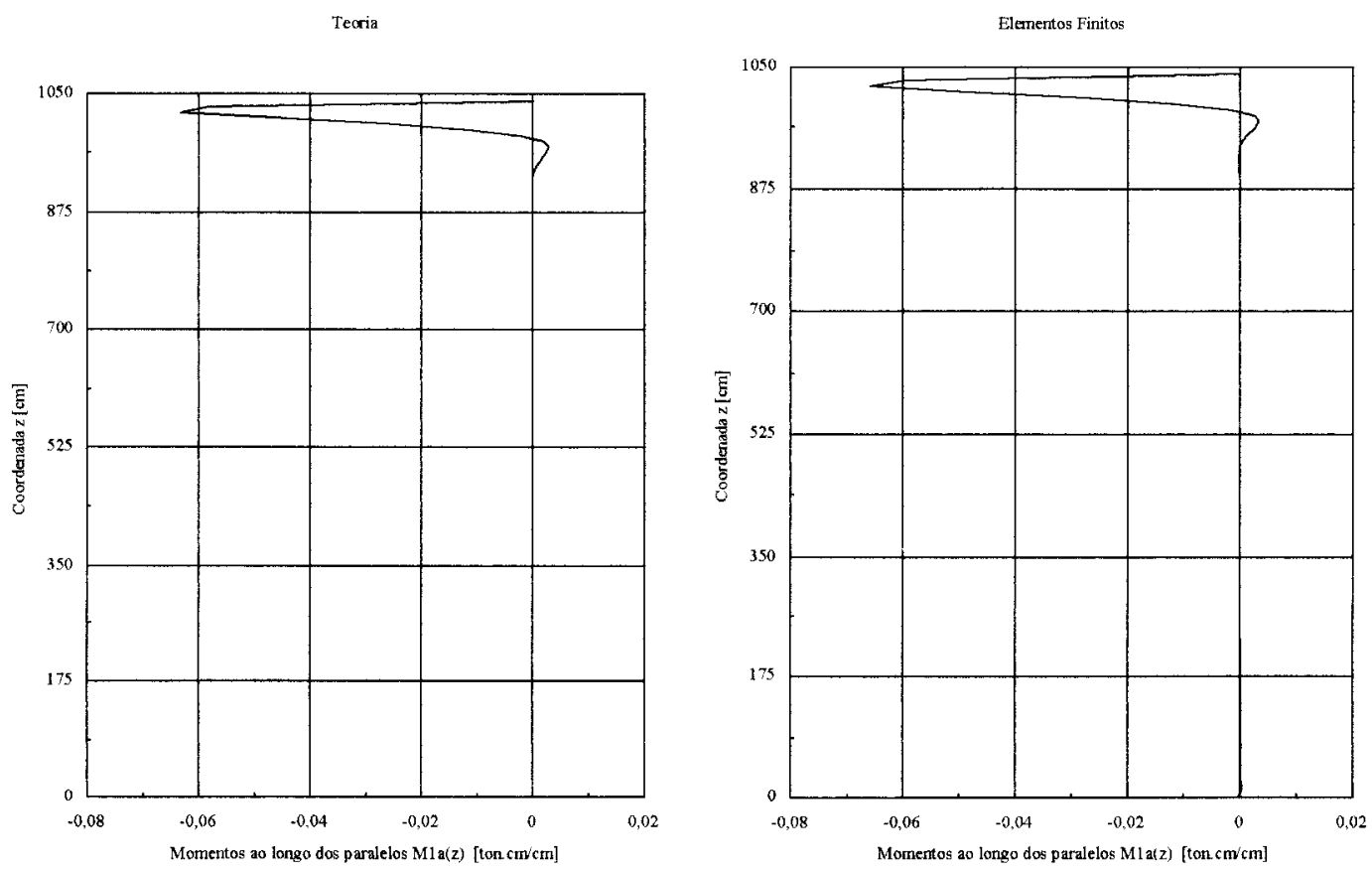

FIGURA 101 - Gráficos para os valores dos momentos ao longo dos paralelos Mla(z) da superfície média da casca cônica
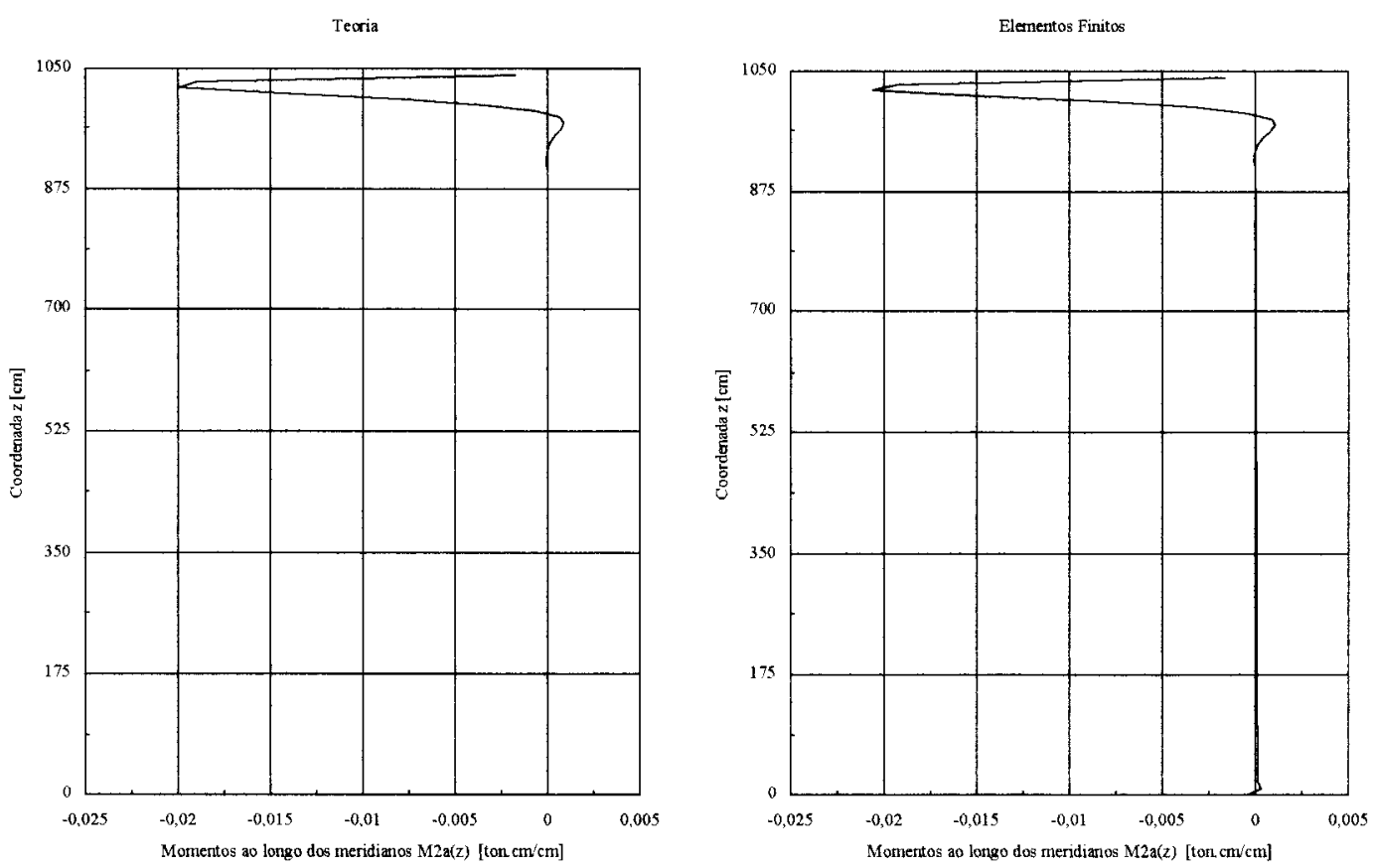

FIGURA 102 - Gráficos para os valores dos momentos ao longo dos meridianos $\mathrm{M} 2 \mathrm{a}(\mathrm{z})$ da superficie média da casca cônica 
Para as tensões atuantes ao longo dos meridianos da superfície interna e externa da casca cônica obteve-se :

TABELA 35 - Valores para as tensões ao longo dos meridianos da superfície interna $\sigma$ lain(z) e externa $\sigma$ laex(z) da casca

\begin{tabular}{|c|c|c|c|}
\cline { 2 - 3 } \multicolumn{1}{c|}{} & \multicolumn{2}{c|}{$\left.\begin{array}{c}\multicolumn{1}{c}{} \\
{[\text { ton ain(z) }}\end{array} \mathrm{cm}^{2}\right]$} & \multicolumn{1}{c}{} \\
\hline $\begin{array}{c}\mathrm{z} \\
{[\mathrm{cm}]}\end{array}$ & Teoria & $\begin{array}{c}\text { Elementos } \\
\text { Finitos }\end{array}$ & Diferença \\
\hline 1039,23 & 0,5300 & 0,4823 & $\mathbf{0 , 0 4 7 7}$ \\
\hline 1030,57 & 0,8823 & 0,8945 & 0,0122 \\
\hline 1021,91 & 0,9112 & 0,9319 & 0,0207 \\
\hline 1013,25 & 0,8091 & 0,8257 & 0,0166 \\
\hline 1004,59 & 0,6868 & 0,6939 & 0,0071 \\
\hline 995,93 & 0,5919 & 0,5904 & 0,0015 \\
\hline 987,27 & 0,5343 & 0,5281 & 0,0062 \\
\hline 978,61 & 0,5069 & 0,4998 & 0,0071 \\
\hline 969,95 & 0,4980 & 0,4922 & 0,0057 \\
\hline 961,29 & 0,4978 & 0,4941 & 0,0037 \\
\hline 952,63 & 0,5003 & 0,4982 & 0,0020 \\
\hline 943,97 & 0,5021 & 0,5010 & 0,0011 \\
\hline 935,31 & 0,5023 & 0,5015 & 0,0008 \\
\hline 926,65 & 0,5010 & 0,5000 & 0,0009 \\
\hline 917,99 & 0,4986 & 0,4974 & 0,0012 \\
\hline
\end{tabular}

\begin{tabular}{|r|r|r|}
\hline \multicolumn{2}{|c|}{$\begin{array}{c}\sigma 1 \mathrm{aex}(\mathrm{z}) \\
\left.\text { [ton/cm }{ }^{2}\right]\end{array}$} & \\
\cline { 1 - 2 } Teoria & $\begin{array}{c}\text { Elementos } \\
\text { Finitos }\end{array}$ & Diferença \\
\hline 0,5300 & 0,4771 & $\mathbf{0 , 0 5 2 9}$ \\
\hline 0,1812 & 0,1769 & 0,0043 \\
\hline 0,1513 & 0,1422 & 0,0091 \\
\hline 0,2495 & 0,2414 & 0,0081 \\
\hline 0,3664 & 0,3631 & 0,0034 \\
\hline 0,4554 & 0,4564 & 0,0010 \\
\hline 0,5070 & 0,5101 & 0,0031 \\
\hline 0,5287 & 0,5314 & 0,0027 \\
\hline 0,5319 & 0,5329 & 0,0011 \\
\hline 0,5264 & 0,5256 & 0,0008 \\
\hline 0,5184 & 0,5162 & 0,0022 \\
\hline 0,5110 & 0,5082 & 0,0029 \\
\hline 0,5051 & 0,5022 & 0,0030 \\
\hline 0,5007 & 0,4980 & 0,0027 \\
\hline 0,4972 & 0,4948 & 0,0024 \\
\hline
\end{tabular}



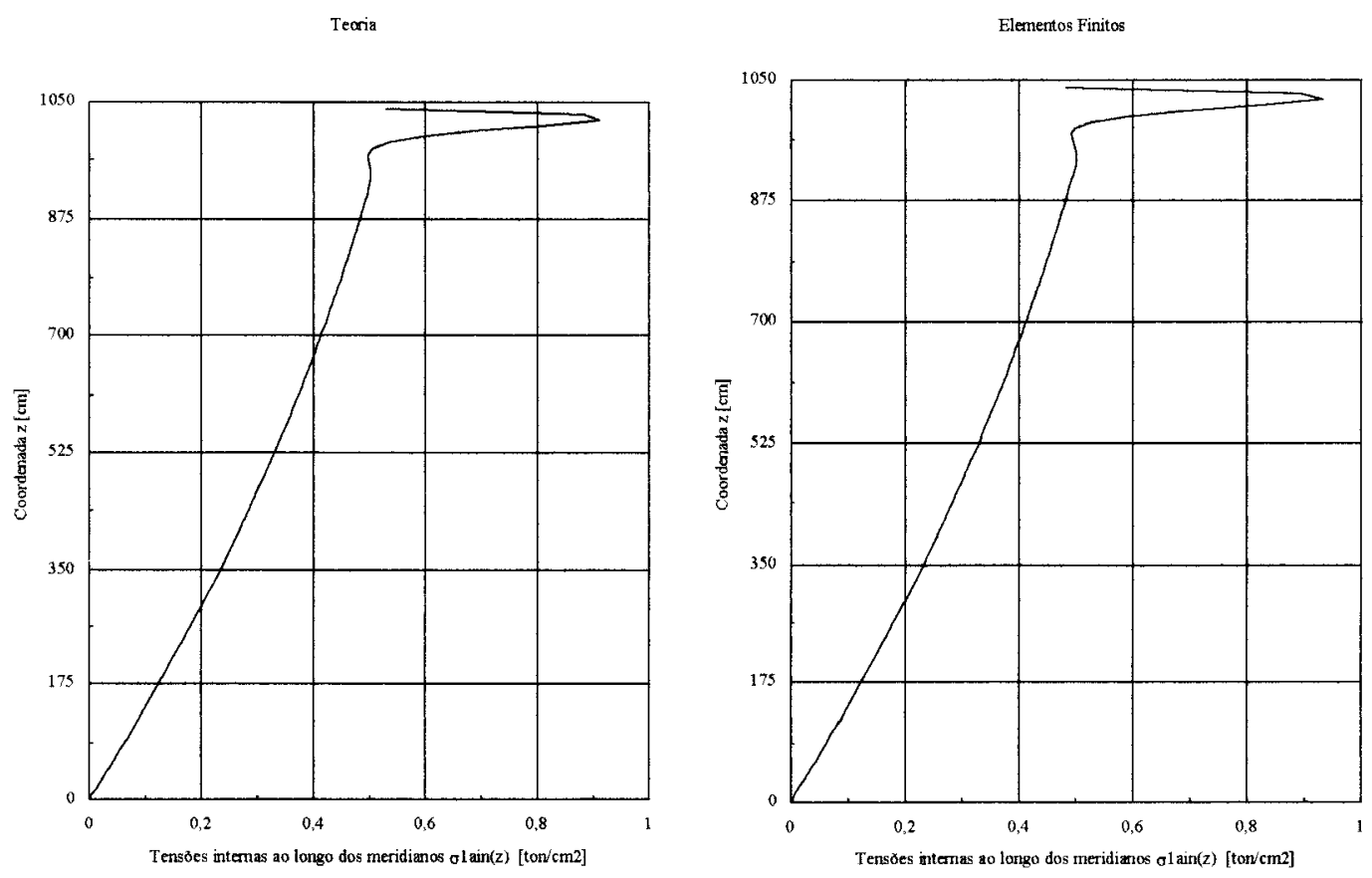

FIGURA 103 - Gráficos para os valores das tensões ao longo dos meridianos da superficie interna $\sigma$ lain(z) da casca cônica
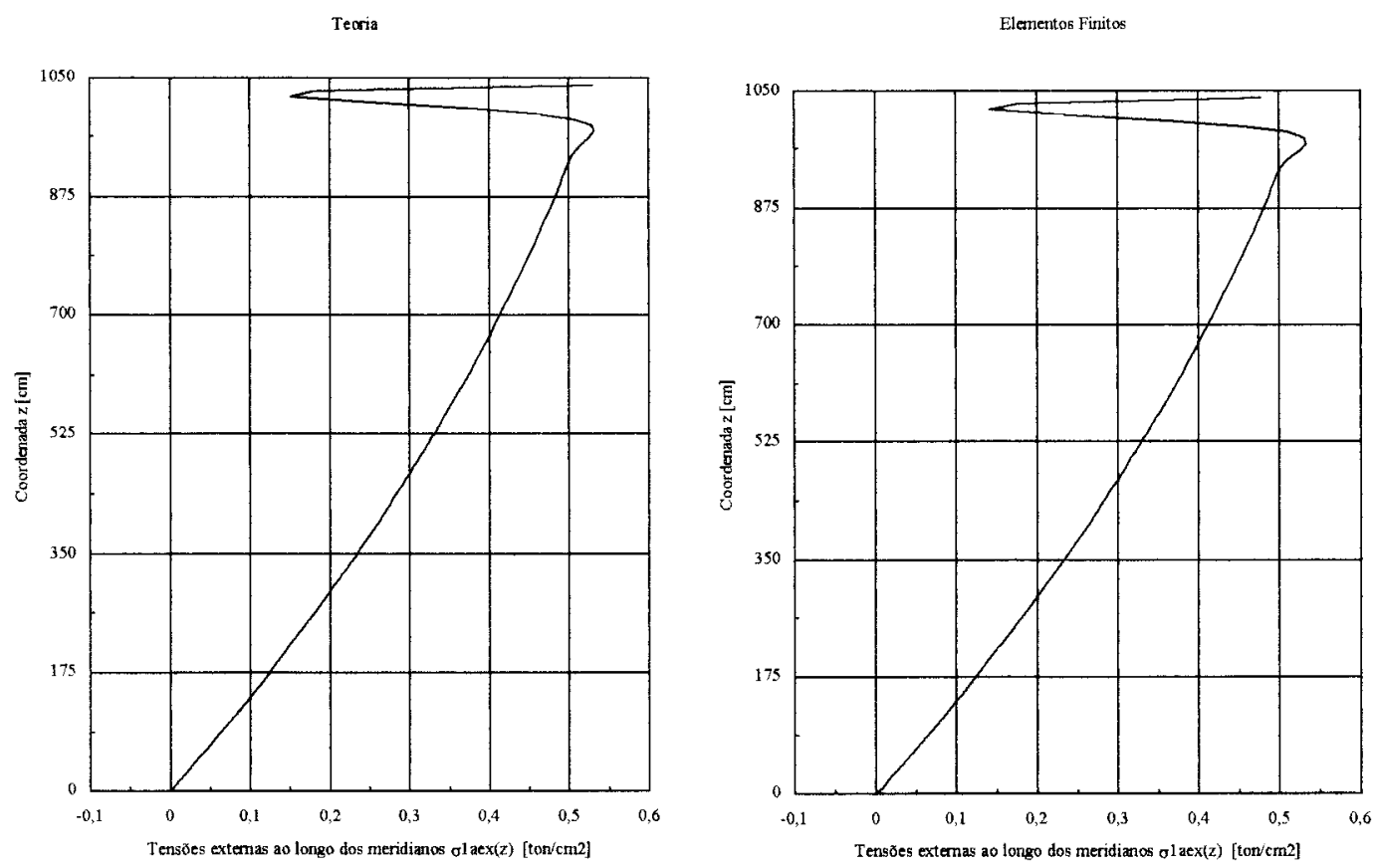

FIGURA 104 - Gráficos para os valores das tensões ao longo dos meridianos da superficie externa $\sigma$ laex(z) da casca cônica 
E, para as tensões atuantes ao longo dos paralelos da superfície interna e externa da casca cônica, encontrou-se :

TABELA 36 - Valores para as tensões ao longo dos paralelos da superficie interna $\sigma 2 \operatorname{ain}(z)$ e externa $\sigma 2 \operatorname{aex}(z)$ da casca

\begin{tabular}{|c|c|c|c|}
\cline { 2 - 3 } \multicolumn{1}{c|}{} & \multicolumn{2}{c|}{$\begin{array}{c}\sigma \text { ain(z) } \\
{\left[\text { ton/ } \mathrm{cm}^{2}\right]}\end{array}$} & \multicolumn{1}{c}{} \\
\hline $\begin{array}{c}\mathrm{z} \\
{[\mathrm{cm}]}\end{array}$ & Teoria & $\begin{array}{c}\text { Elementos } \\
\text { Finitos }\end{array}$ & Diferença \\
\hline 1039,23 & 0,1711 & 0,1538 & $\mathbf{0 , 0 1 7 3}$ \\
\hline 1030,57 & 0,5808 & 0,5767 & 0,0041 \\
\hline 1021,91 & 0,8074 & 0,8101 & 0,0027 \\
\hline 1013,25 & 0,8984 & 0,9048 & 0,0064 \\
\hline 1004,59 & 0,9112 & 0,9169 & 0,0056 \\
\hline 995,93 & 0,8905 & 0,8926 & 0,0021 \\
\hline 987,27 & 0,8627 & 0,8611 & 0,0016 \\
\hline 978,61 & 0,8399 & 0,8358 & 0,0041 \\
\hline 969,95 & 0,8251 & 0,8199 & 0,0052 \\
\hline 961,29 & 0,8171 & 0,8120 & 0,0051 \\
\hline 952,63 & 0,8134 & 0,8090 & 0,0045 \\
\hline 943,97 & 0,8119 & 0,8081 & 0,0038 \\
\hline 935,31 & 0,8109 & 0,8076 & 0,0033 \\
\hline 926,65 & 0,8098 & 0,8067 & 0,0030 \\
\hline 917,99 & 0,8082 & 0,8052 & 0,0030 \\
\hline
\end{tabular}

\begin{tabular}{|r|r|r|}
\hline \multicolumn{2}{|c|}{$\begin{array}{c}\sigma 2 \mathrm{aex}(\mathrm{z}) \\
\left.\text { [ton/ } \mathrm{cm}^{2}\right]\end{array}$} & \multicolumn{1}{|c}{} \\
\cline { 1 - 2 } Teoria & $\begin{array}{c}\text { Elementos } \\
\text { Finitos }\end{array}$ & Diferença \\
\hline 0,1503 & 0,1340 & $\mathbf{0 , 0 1 6 3}$ \\
\hline 0,3532 & 0,3457 & 0,0075 \\
\hline 0,5685 & 0,5631 & 0,0054 \\
\hline 0,7252 & 0,7247 & 0,0005 \\
\hline 0,8135 & 0,8164 & 0,0028 \\
\hline 0,8498 & 0,8530 & 0,0032 \\
\hline 0,8554 & 0,8569 & 0,0015 \\
\hline 0,8473 & 0,8464 & 0,0009 \\
\hline 0,8359 & 0,8330 & 0,0029 \\
\hline 0,8260 & 0,8220 & 0,0040 \\
\hline 0,8190 & 0,8148 & 0,0043 \\
\hline 0,8146 & 0,8105 & 0,0041 \\
\hline 0,8117 & 0,8080 & 0,0037 \\
\hline 0,8096 & 0,8063 & 0,0033 \\
\hline 0,8078 & 0,8047 & 0,0031 \\
\hline
\end{tabular}



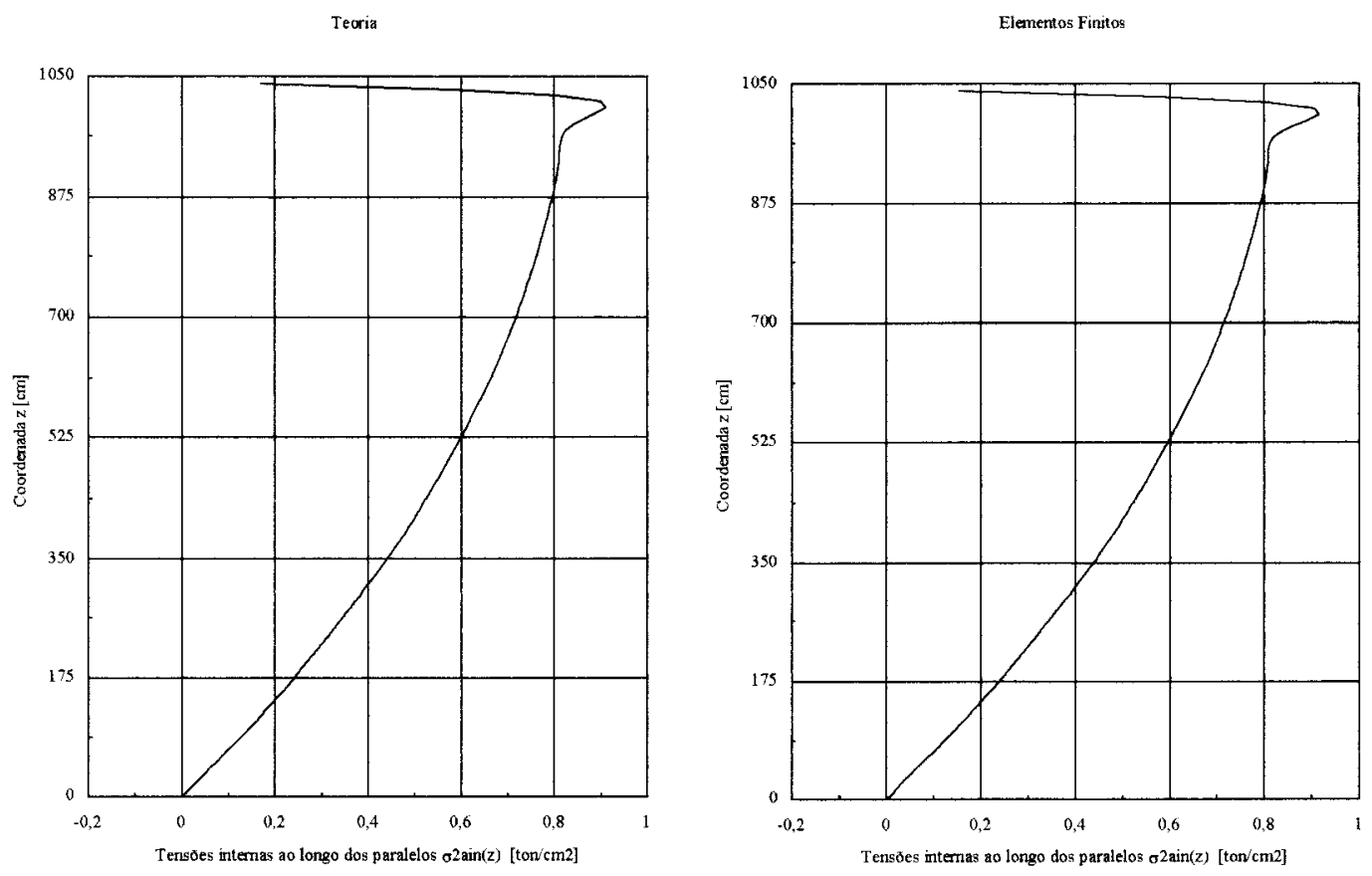

FIGURA 105 - Gráficos para os valores das tensões ao longo dos paralelos da superficie interna $\sigma 2$ ain( $(\mathrm{z})$ da casca cônica
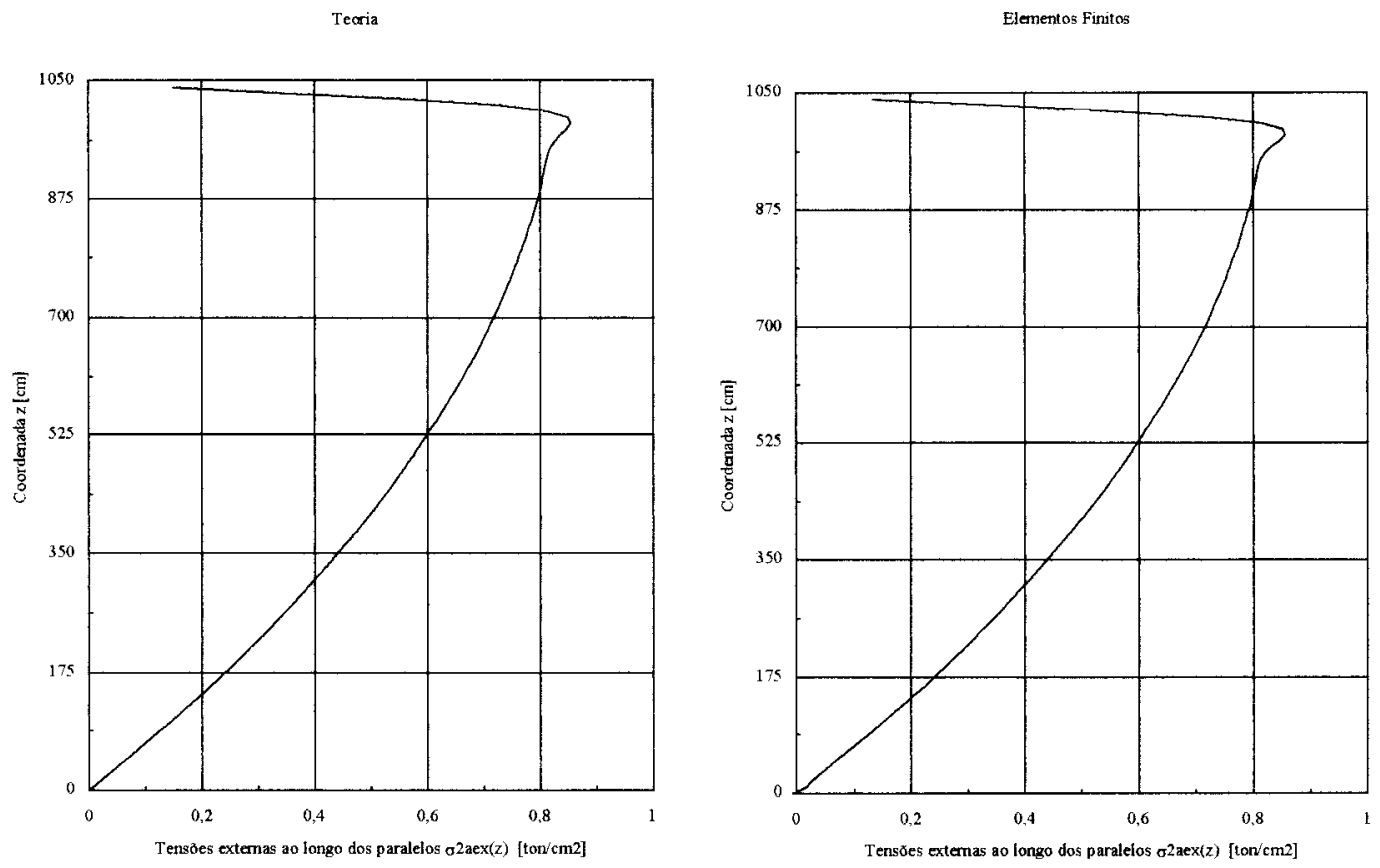

FIGURA 106 - Gráficos para os valores das tensões ao longo dos paralelos da superficie externa $\sigma 2 \mathrm{aex}(\mathrm{z})$ da casca cônica 
Observar, nos gráficos abaixo, que as tensões teóricas ao longo dos meridianos e paralelos da casca cônica, como para borda engastada, possuem seus valores praticamente coincidêntes com as tensões de membrana para a região abaixo de $z=H-\lambda z=926,51[\mathrm{~cm}]$.

Teoria

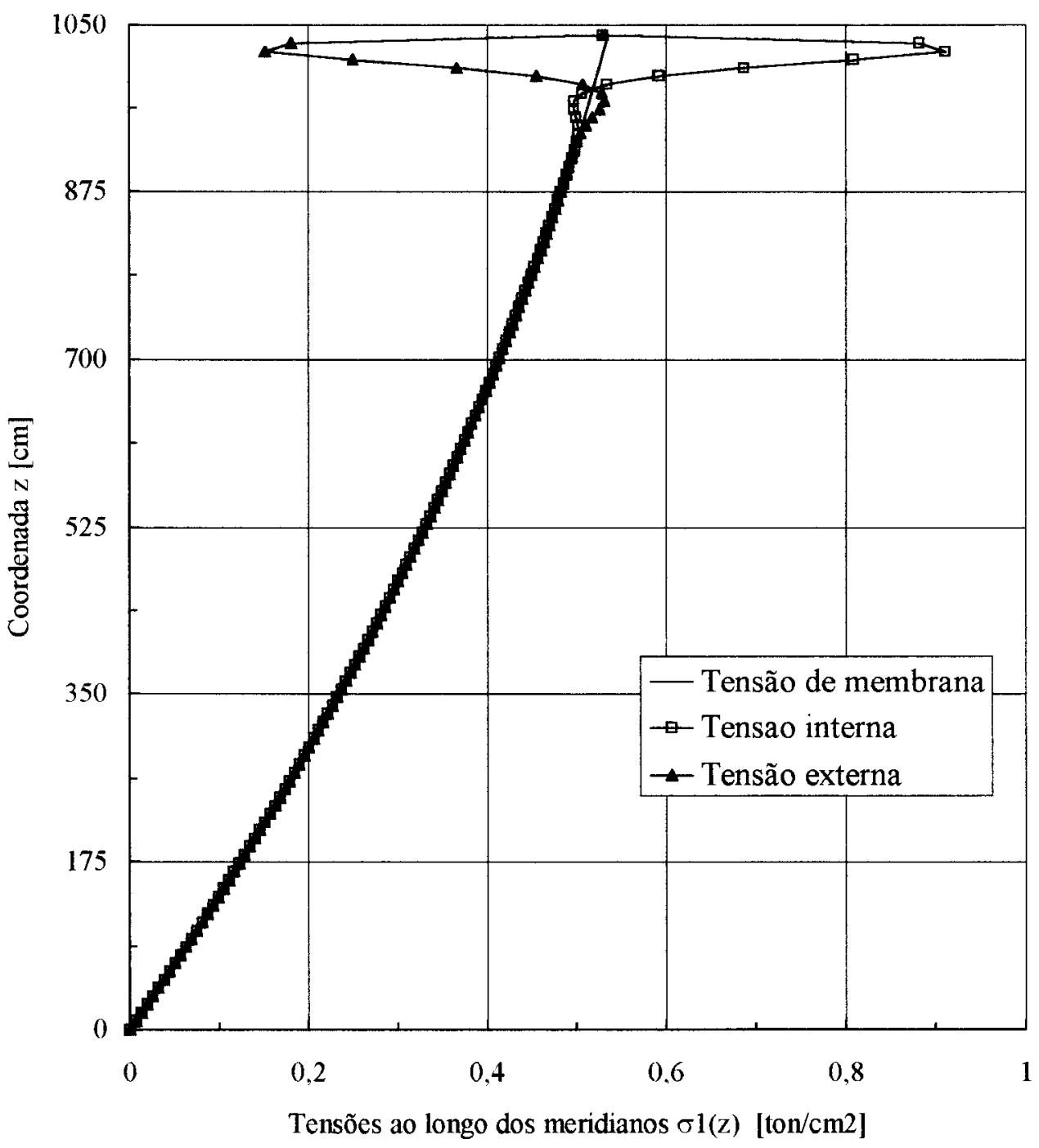

FIGURA 107 - Gráfico comparativo para os valores das tensões ao longo dos meridianos da casca cônica : $\sigma \operatorname{lm}(\mathrm{z}), \sigma \mathrm{lain}(\mathrm{z})$ e $\sigma \mathrm{laex}(\mathrm{z})$ 
Teoria

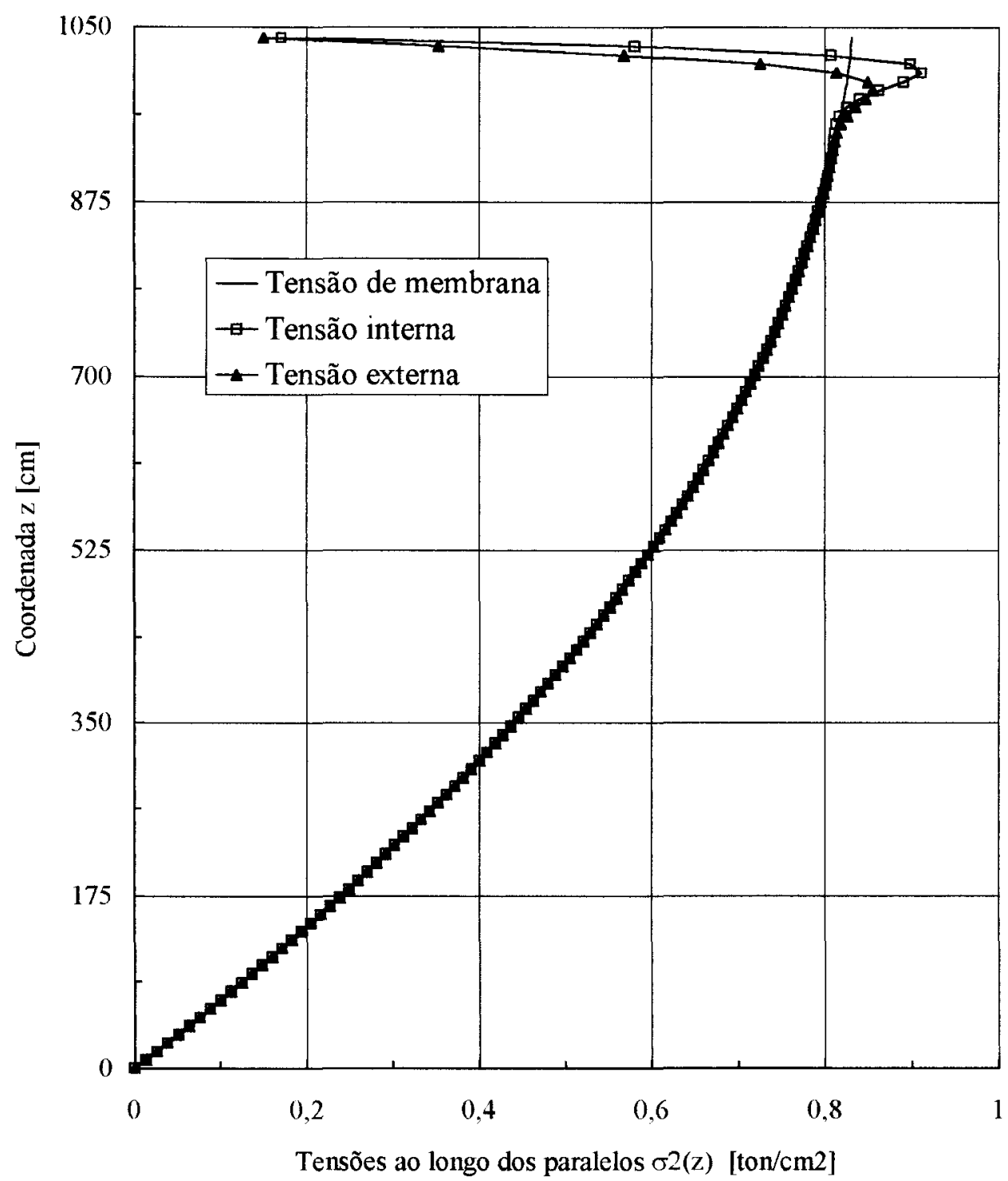

FIGURA 108 - Gráfico comparativo para os valores das tensões ao longo dos paralelos da casca cônica : $\sigma 2 \mathrm{~m}(\mathrm{z}), \sigma 2 \mathrm{ain}(\mathrm{z})$ e $\sigma 2 \mathrm{aex}(\mathrm{z})$ 


\section{CONCLUSÕES}

Muitos estudos tem explorado o assunto referente ao comportamento das cascas de revolução, principalmente das cascas cilíndricas, sendo que muitos desses trabalhos surgem da busca por um tratamento teórico que possa prever as tensões na estrutura real.

Atualmente existem recursos computacionais sofisticados que facilitam o trabalho da predição do comportamento das estruturas, mas normalmente não são acessíveis aos engenheiros não acadêmicos, que, na verdade, são os que convivem diariamente com problemas de projeto estrutural. Este trabalho tentou introduzir um equacionamento teórico aproximado para ser utilizado por esse engenheiro.

A comparação dos resultados obtidos através das expressões teóricas aproximadas (capítulo 2) com os obtidos através da análise por elementos finitos (capítulo 3) foi a forma encontrada para defrontar duas ferramentas diferentes de cálculo estrutural, mostrando que é possível, dentro de hipóteses bem definidas, vislumbrar o comportamento das cascas cilíndricas e cônicas através da utilização de uma boa calculadora científica ou de um simples programa de planilha eletrônica.

Para a casca cilíndrica nada se apresentou de novidade, já que a solução teórica simplificada para seu comportamento, dentro das hipóteses obedecidas, é facilmente encontrada em várias das referências bibliográficas citadas (capítulo 7)

Para as cascas cônicas pouco ou quase nada encontrou-se referente a soluções teóricas aproximadas prontas para o uso do engenheiro de projetos.

Através das orientações de BELLUZZI, transformando-se a casca cônica em casca esférica equivalente, encontrou-se equações, para deslocamentos, rotações, momentos e tensões, de aplicação relativamente fácil no dia a dia do projetista. 
Obviamente era esperado que os resultados obtidos, tanto por um desenvolvimento teórico, como pelo método dos elementos finitos, fossem idênticos, porém, não se esperava que a solução de uma casca cônica utilizando-se como modelo uma esférica equivalente pudesse levar a um equacionamento aceitável para a sua aplicação na engenharia. $O$ estudo de forma comparativa com o método dos elementos finitos permitiu calibrar a solução teórica aproximada desenvolvida.

Apresentou-se, portanto, um equacionamento teórico aproximado que muito se assemelha a resolução pelo metódo dos elementos finitos.

Isto pode ser observado através das colunas "Diferença" das tabelas do capítulo 5, que mostram como são pequenos os valores encontrados para as diferenças absolutas entre os parâmetros obtidos pelas equações e os obtidos por elementos finitos.

Para a casca cilíndrica pode-se dizer que, nas condições consideradas, os resultados são muito bons para qualquer das ferramentas de cálculo que se deseje utilizar.

Para as cascas cônicas devem ser consideradas as limitações comentadas por BELLUZZI quanto aos resultados da solução aproximada serem aceitáveis para ângulos $\theta \mathrm{r}$ maiores que 20 [graus] e, não menores que 10 [graus] no caso da relação $\mathbf{R} / \mathbf{t}$ ser muito grande. Segundo ele a aproximação é tanto melhor quanto mais rápido é o amortecimento das "perturbações de borda" ao longo da casca.

Para visualizar esta limitação calculou-se uma casca cônica com as mesmas dimensões das do capítulo 5 , com sua borda superior engastada e com ângulo de inclinação com a horizontal de 10 [graus] obtendo-se as seguintes diferenças absolutas máximas que foram consideradas elevadas comparativamente com as das cascas com 30, 45 e 60 [graus] de inclinação : 
TABELA 37 - Valores das diferenças absolutas máximas para os parâmetros da casca cônica com a borda superior engastada e inclinação $\theta \mathrm{r}=10$ [graus]

\begin{tabular}{|c|c|c|c|c|c|c|c|}
\hline & \multicolumn{2}{|c|}{$\begin{array}{l}\xi \mathrm{e}(\mathrm{z}) \\
{[\mathrm{cm}]}\end{array}$} & & & \multicolumn{2}{|c|}{$\begin{array}{c}\phi \mathrm{e}(\mathrm{z}) \\
\text { [radianos] }\end{array}$} & \\
\hline $\begin{array}{c}\mathrm{z} \\
{[\mathrm{cm}]}\end{array}$ & Teoria & Finitos & Diferença & $\begin{array}{c}\mathrm{z} \\
{[\mathrm{cm}]}\end{array}$ & Teoria & Finitos & Diferença \\
\hline 75,82 & 0,5586 & 0,5445 & 0,0141 & 100,51 & $-0,0665$ & $-0,0700$ & 0,0035 \\
\hline & \multicolumn{2}{|c|}{$\begin{array}{c}\operatorname{Mle}(\mathrm{z}) \\
{[\text { ton } \mathrm{cm} / \mathrm{cm}]}\end{array}$} & & & \multicolumn{2}{|c|}{$\begin{array}{c}\mathrm{M} 2 \mathrm{e}(\mathrm{z}) \\
{[\text { ton } \mathrm{cm} / \mathrm{cm}]}\end{array}$} & \\
\hline $\begin{array}{c}\mathrm{z} \\
{[\mathrm{cm}]}\end{array}$ & Teoria & Finitos & Diferença & $\begin{array}{c}\mathrm{z} \\
{[\mathrm{cm}]}\end{array}$ & Teoria & Finitos & Diferença \\
\hline \multirow[t]{2}{*}{105,80} & 0,9294 & 0,9670 & 0,0376 & 105,80 & 0,2739 & 0,2901 & 0,0162 \\
\hline & \multicolumn{2}{|c|}{$\begin{array}{l}\sigma \text { lein }(\mathrm{z}) \\
{\left[\text { ton } / \mathrm{cm}^{2}\right]}\end{array}$} & & & \multicolumn{2}{|c|}{$\begin{array}{l}\sigma l e e x(z) \\
{\left[\text { ton } / \mathrm{cm}^{2}\right]}\end{array}$} & \\
\hline $\begin{array}{c}\mathrm{z} \\
{[\mathrm{cm}]}\end{array}$ & Teoria & Finitos & Diferença & $\begin{array}{c}\mathrm{z} \\
{[\mathrm{cm}]}\end{array}$ & Teoria & Finitos & Diferença \\
\hline \multirow[t]{2}{*}{105,80} & $-3,6892$ & $-3,9351$ & 0,2459 & 105,80 & 7,4637 & 7,6691 & 0,2054 \\
\hline & \multicolumn{2}{|c|}{$\begin{array}{c}\sigma 2 \operatorname{ein}(z) \\
{\left[\operatorname{ton} / \mathrm{cm}^{2}\right]}\end{array}$} & & & \multicolumn{2}{|c|}{$\begin{array}{c}\sigma 2 \operatorname{eex}(\mathrm{z}) \\
{\left[\text { ton } / \mathrm{cm}^{2}\right]}\end{array}$} & \\
\hline $\begin{array}{c}\mathrm{z} \\
{[\mathrm{cm}]}\end{array}$ & Teoria & Finitos & Diferença & $\begin{array}{c}\mathrm{z} \\
{[\mathrm{cm}]}\end{array}$ & Teoria & Finitos & Diferença \\
\hline 103,15 & 0,3966 & 0,1847 & 0,2119 & 99,62 & 1,5638 & 1,4316 & 0,1322 \\
\hline
\end{tabular}

Para $\theta r=10$ [graus] encontrou-se o comprimento de amortecimento $\lambda_{\text {cone }}$ $=287,99[\mathrm{~cm}]$, que na vertical é $\lambda z=50,01[\mathrm{~cm}]$, representando quase $50 \%$ da altura total da casca cônica $\mathrm{H}=105,80$ [cm], reforçando a observação de BELLUZZI quanto aos limites de aplicação das equações da teoria aproximada.

Finalmente concluindo, as equações desenvolvidas neste trabalho, para cascas cilíndricas e cônicas com bordas engastadas ou apoiadas fixas sob a ação de pressão hidrostática, podem ser utilizadas para prever o comportamento estrutural das mesmas, desde que se observe as hipóteses fundamentais de "Kirchoff-Love" (ver capitulo 2) e o limite de inclinação para as cascas cônicas recomendados por BELLUZZI.

Esta distante a simulação do comportamento real das cascas, já que após construídas, em campo, encontram-se inúmeros outros parâmetros que não foram considerados neste trabalho e que deverão fazer parte de futuros estudos para 
sua continuidade, como por exemplo : plastificação do aço, deformações geométricas iniciais de fabricação, ocorrência de mudanças bruscas de espessura, imperfeições iniciais e tensões residuais devido ao processo de soldagem, descontinuidade na região da união cilindro "versus" cone, etc.

Apesar disso, procurou-se apresentar um melhor esboço do comportamento estrutural das cascas, do que a simples utilização da teoria de membrana sem a consideração da flexão causada pelas "perturbações de borda". 


\section{BIBLIOGRAFIA}

1. AMERICAN INSTITUTE OF STEEL CONSTRUCTION (1980a). Mamual of Steel Construction. $8^{\mathrm{a}}$ edição. New York. AISC.

2. AMERICAN SOCIETY OF MECHANICAL ENGINEERS (1960). Pressure Vessel and Piping Design. Collected Papers. New York.

3. AMERICAN SOCIETY OF MECHANICAL ENGINEERS (1968a). ASME Boiler and Pressure Vessel Code Section VIII: Rules for construction of pressure vessels - Division 1. New York.

4. ASSOCIAÇÃO BRASILEIRA DE NORMAS TÉCNICAS (1978a). NB-89. Tanques soldados para armazenamento de petróleo e derivados. Rio de Janeiro.

5. ASSOCIAÇÃO BRASILEIRA DE NORMAS TÉCNICAS (1984a). NB-8400/84. Cálculo de equipamento para levantamento e movimentação de cargas. Rio de Janeiro.

6. ASSOCIAÇÃO BRASILEIRA DE NORMAS TÉCNICAS (1986a). NBR 8800/86. Projeto e execução de estruturas de aço de edifícios:método dos estados limites. Rio de Janeiro.

7. ASSOCIAÇÃO BRASILEIRA DE NORMAS TÉCNICAS (1988). NBR 6123/88. Forças devidas ao vento em edificações. Rio de Janeiro

8. BADIALE, R.C. (1996b). Estudo de flambagem de cascas cilíndricas submetidas à compressão axial - Enfase: cascas metálicas. Seminário da Disciplina SET819 Instabilidade das Estruturas. São Carlos, Curso de Pós-Graduação em Engenharia de Estruturas da Escola de Engenharia de São Carlos, Universidade de São Paulo, Agosto 
9. BADIALE, R.C., CALIL Jr.,C. (1996a). CRA071 - Projeto e construção de um silo para açucar. In: XXV CONGRESSO BRASILEIRO DE ENGENHARIA AGRÍCOLA, Bauru, Julho

10.BEDNAR, H. H. (1986b). Pressure vessels design handbook. 2. ed. New York, Van Nostrand.

11.BELLUZZI, O. (1967), Ciencia de la construccion. Madrid, Aguilar, v.3.

12.BLODGETT, O. W. (1966). Design of Welded Structures. Cleveland. The James F. Lincoln Arc Welding Foundation.

13.BROWNELL, L. E., YOUNG, E. H. (1959). Process Equipment Design. New York, John Wiley.

14.BRUSH, D. O., ALMROTH, B. O. (1975). Buckling of Bar, Plates, and Shells. New York. McGraw-Hill.

15.BULL, J. W. (1990). Finite element applications to thin-walled structures. New York, Elsevier.

16.BUSHNELL, D. (1989a). Computerized Buckling Analysis of Shells. Dordrecht, Kluwer Academic.

17.CHAJES, A. (1974). Principles of Structural Stability Theory. New Jersey, Prentice-Hall.

18. COOPERATIVA CENTRAL DOS PRODUTORES DE AÇUCAR E ALCOOL DO ESTADO DE SÃO PAULO (1983a). I Seminário de Tecnologia Industrial. Piracicaba. Centro de Tecnologia Copersucar.

19.COOPERATIVA CENTRAL DOS PRODUTORES DE AÇUCAR E ALCOOL DO ESTADO DE SÃO PAULO (1987a). III Seminário de Tecnologia Industrial. Piracicaba. Centro de Tecnologia Copersucar.

20.DEUTSCHE NORM. (1987b). DIN 1055. Design loads for buildings: loads in silo bins. Part 6. Berlin, DIN - Sprachendienst.

21.DONNEL, L. H. (1934). A new theory for the buckling of thin cylinders under axial compression and bending. Trans. of ASME, v.56, p.795-806, Nov.

22.EUROPEAN CONVENTION FOR CONSTRUCTIONAL STEELWORK. (1976). Shells. In.: Manual on Stability of Steel Structures. $2^{\mathrm{a}}$ Edition. Milano, Tip. Scotti-Cornate d'A. cap.10, p.275-297, June. 


\section{EUROPEAN CONVENTION FOR CONSTRUCTIONAL STEELWORK.} (1983b). ECCS Recommendations for Steel Construction: buckling of shells. $2^{\mathrm{a}}$ Edition

24.FLUGGE, W. (1973). Stresses in Shells. 2a Edição. New York, Springer-Verlag. 25.FUNG, Y. C., SECHLER, E. E. (1957a). Buckling of thin-walled circular cylinders under axial compression and internal pressure. Journal of the Aeronautical Sciences, v.24, n.5, p.351-356, May.

26.GAYLORD, E. H., GAYLORD, C. N. (1984b). Design of steel bins for storage of bulk solids. New Jersey, Prentice-Hall.

27.HARRIS, L. A et al. (1957b). The stability of thin-walled unstiffened circular cylinders under axial compression including the effects of internal pressure. Journal of the Aeronautical Sciences, v.24, n.8, p.587-596, Aug.

28.KANEMITSU, S., NOJIMA, N. M. (1939). Axial Compression Tests of Thin Circular Cylinders. California. M. S. Thesis. Dept. of Aeronautical Engineering, California Institute of Technology.

29. MANFRIM, I. M. S. (1994a). Um estudo dos silos para açúcar : propriedades fisicas do material armazenado, recomendações construtivas, normativas e análise estrutural. São Carlos. 81p. Dissertação (Mestrado). Escola de Engenharia de São Carlos, Universidade de São Paulo.

30.McLEAN, R. F. (1984c). Safer Silos. In.: RHODES, J., WALKER, A.C. Developments in Thin-Walled Structures 2. London, Elsevier, Cap. 6, p. 211-237.

31.MUKHANOV, K. (1980b). Estruturas metálicas. Moscou, Editora Mir.

32. ODLAND, J. (1978b). Bucking resistence of unstiffened and stiffened circular cylindrical shell structures. Norwegian Maritime Research, v.6, n.3, p.2-22.

33.PIROK, J. N., WOZNIAK, R. S. (1968b). Steel Tanks. In.: GAYLORD, E. H., GAYLORD, C. N. Structural Engineering Handbook. New York, McGraw-Hill. section 23, p.23.1-23.26.

34.PISARENKO, G. S.; YÁKOVLEV, A. P.; MATVÉEV, V. V. (1979). Cálculo de bóvedas de paredes delgadas. In: Manual de resistencia de materiales. Moscú, Editorial Mir, Cap.16, p.425-436. 
35.ROTTER, J. M. (1985a). Buckling under axial compression. In: Design of steel bins for the storage of bulk solids. Sydney, University of Sidney.

36.ROTTER, J. M. (1985b). Design of steel bins for the storage of bulk solids. Sydney, University of Sidney.

37.SÁlES, J. J. , MALITE, M., GOLÇALVES, R. M. (1994b). SET 403 - Sistemas estruturais - Elementos estruturais. São Carlos, Escola de Engenharia de São Carlos, Universidade de São Paulo.

38.SAP90 ETABS SAFE (1995). Computer software for structural and earthquake engineering : Installation guide. Berkeley. Computers and Structures, Inc..

39. SHULTE, H., YAGUI, T. (1981). Estruturas de Aço : elementos básicos. São Carlos. Escola de Engenharia de São Carlos, Universidade de São Paulo.

40.TENG, J. G., ROTTER, J. M. (1989b). Plastic collapse of restrained steel silo hoppers. Inl. Construct. Steel Research, n. 14, p.139-158.

41.TENG, J. G., ROTTER, J. M. (1991). Plastic buckling of rings at steel silo transition junctions. Jnl. Construct. Steel Research, n. 19, p. 1-18.

42.TENG, J. G., ROTTER, J. M. (1992). Recent research on the behaviour and design of steel silo hoppers and transition junctions. Jnl. Construct. Steel Research, n.23, p.313-343.

43.TIMOSHENKO, S. P. (1961). Theory of Elastic Stability. Tokio. McGraw-Hill.

44.TIMOSHENKO, S. P. Resistência dos Materiais. 02 Volumes. Brasil. LTC Editora.

45.TIMOSHENKO, S. P., GOODIER, J. N. (1980c). Teoria da Elasticidade. $3^{\mathrm{a}}$ edição. Rio de Janeiro. Guanabara Dois.

46.VON KÁRMÁN, T., DUNN, L. G., TSIEN, H. (1940). The influence of curvature on the buckling characteristics of structures. Journal of the Aeronautical Sciences, v.7, n.7, p.276-289, May.

47. VON KÁRMÁN, T., TSIEN, H. (1941). The buckling of thin cylindrical shells under axial compression. Journal of the Aeronautical Sciences, v.8, n.8, p.303312 , June. 
48.WEINGARTEN, V. I., MORGAN, E. J., SEIDE, P. (1965a). Elastic stability of thin-walled cylindrical and conical shells under axial compression. $A I A A J n l, \mathrm{v} .3$, n. 3, p.500-505, March.

49.WEINGARTEN, V. I., MORGAN, E. J., SEIDE, P. (1965b). Elastic stability of thin-walled cylindrical and conical shells under combined internal pressure and axial compression. AIAA Jnl, v. 3, n.6, p.1118-1125, June. 